\title{
Synthesis of Trifluoromethyl-Allenes by Gold-Catalyzed Rearrangement of Propargyl Benzyl Ethers
}

\author{
Arnaud Boreux, ${ }^{\mathrm{a}, \mathrm{b}}$ Geoffroy H. Lonca, ${ }^{\mathrm{a}}$ Olivier Riant, ${ }^{, \mathrm{b}}$ and Fabien Gagosz,,a \\ ${ }^{a}$ Laboratoire de Synthèse Organique, UMR 7652 CNRS/Ecole Polytechnique, Ecole Polytechnique, Route de \\ Saclay, 91128 Palaiseau, France \\ ${ }^{b}$ Institute of Condensed Matter and Nanosciences (IMCN), division of MOlecules, Solids and ReactiviTy \\ (MOST), Université catholique de Louvain, Place Louis Pasteur 1, bte L4.01.02, 1348 Louvain-la-Neuve, \\ Belgique
}

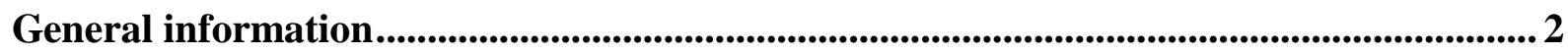

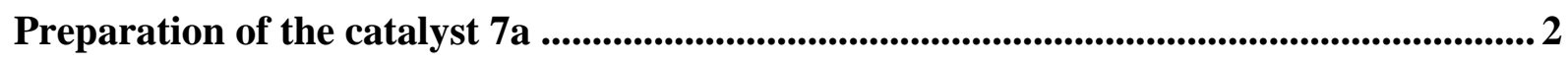

Preparation of starting materials...................................................................................................... 3

Catalysis step .................................................................................................................................................... 27

Post-functionalization of the $\mathrm{CF}_{3}$-allenes ...................................................................4 46

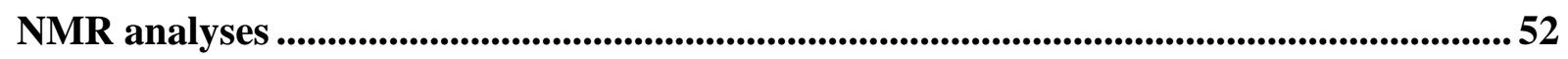




\section{General information}

Unless otherwise noted, all reactions for the synthesis of substrates were performed under dry oxygen-free argon or nitrogen atmosphere. The scope of the gold-catalyzed reaction was carried out using deuterated chloroform dried on molecular sieves. Gold-catalyzed reactions were performed in an NMR tube using dichloroethane as an internal standard. Thin Layer Chromatography were performed on aluminium plates bearing a $0.25 \mathrm{~mm}$ of Merck Silica Gel $60 \mathrm{~F}_{254}$, visualized by fluorescence quenching at $254 \mathrm{~nm}$ and chemical revelation using acidic solution of para-anisaldehyde or basic solution of potassium permanganate. Flash chromatography was performed using silica gel $60(40-63 \mu \mathrm{m})$. Commercial reagents were purchased from Acros, Fluorochem, TCI, Sigma-Aldrich, Alfa-Aesar and used as received. NMR analysis was performed at room temperature on Bruker DPX 300 MHz Fourier Transform Spectrometer operating at $300 \mathrm{MHz} / 400 \mathrm{MHz}$ for ${ }^{1} \mathrm{H}$ and $75 \mathrm{MHz} / 100 \mathrm{MHz}$ for ${ }^{13} \mathrm{C}$. Residual solvent peaks of $\mathrm{CDCl}_{3}$ were used as internal references: $7.26 \mathrm{ppm}$ for ${ }^{1} \mathrm{H}$ spectra and 77.16 ppm for ${ }^{13} \mathrm{C}$ spectra. The following abbreviations were used in order to describe de peaks multiplicities: $\mathrm{s}=$ singlet, $\mathrm{d}=$ doublet, $\mathrm{t}=$ triplet, $\mathrm{q}=$ quartet, quint $=$ quintuplet, hex = hexuplet, hept $=$ heptuplet, $\mathrm{m}=$ multiplet, $\mathrm{br}=$ broad. High resolution mass spectra were recorded by on a JMS-GCmateII mass spectrometer or on a Thermo Scientific QExactive. Infrared absorption spectra were recorded as a liquid deposition on a ZnSe crystal on a Shimadzu FTIR 8400 Spectrophotometerspectra or in solutions in $\mathrm{CCl}_{4}$ or $\mathrm{CDCl}_{3}$ using $\mathrm{NaCl}$ cells on a Perkin-Elmer FT 2000 or Perkin-Elmer Spectrum Two.

\section{Preparation of the catalyst 7a}

The phosphonite ligand as well as the corresponding $\mathrm{LAuCl}$ complex were prepared according to a literature procedure. ${ }^{1}$

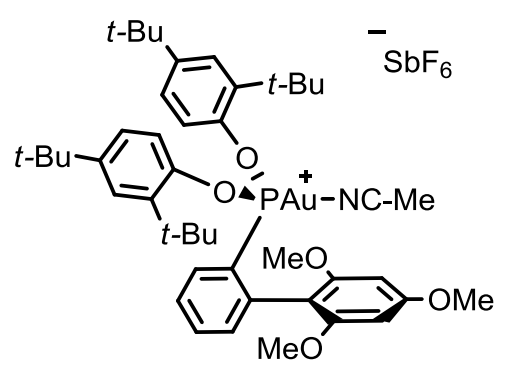


(0.1 mol.L $\left.\mathrm{L}^{-1}\right)$ under nitrogen. The mixture was stirred for $15 \mathrm{~min}$ at room temperature in the dark, then filtered on a pad of Celite ${ }^{\circledR}$. The solvents were evaporated, then the complex 7a was washed with pentane to be obtained as a white solid.

$\begin{array}{ll}{ }^{1} \mathbf{H ~ N M R}(\delta, \mathrm{ppm}) & 8.19-8.14(\mathrm{~m}, 1 \mathrm{H}), 7.71(\mathrm{t}, J=7.5 \mathrm{~Hz}, 1 \mathrm{H}), 7.60(\mathrm{t}, J=7.5 \mathrm{~Hz}, 1 \mathrm{H}), \\ \left(400 \mathrm{MHz}, \mathrm{CDCl}_{3}\right) & 7.38(\mathrm{~s}, 2 \mathrm{H}), 7.30(\mathrm{t}, J=7.8 \mathrm{~Hz}, 1 \mathrm{H}), 7.20-7.18(\mathrm{~m}, 4 \mathrm{H}), 6.35(\mathrm{~s}, \\ & 2 \mathrm{H}), 3.95(\mathrm{~s}, 3 \mathrm{H}), 3.63(\mathrm{~s}, 6 \mathrm{H}), 2.41(\mathrm{~s}, 3 \mathrm{H}), 1.30(\mathrm{~s}, 2 \times 18 \mathrm{H})\end{array}$

${ }^{1} \mathbf{H}$ NMR $(\delta, \mathrm{ppm}) \quad 8.17-8.13(\mathrm{~m}, 1 \mathrm{H}), 7.74(\mathrm{t}, J=7.6 \mathrm{~Hz}, 1 \mathrm{H}), 7.62(\mathrm{t}, J=7.7 \mathrm{~Hz}, 1 \mathrm{H})$, $\left(400 \mathrm{MHz}, \mathrm{CD}_{2} \mathrm{Cl}_{2}\right) \quad 7.43(\mathrm{~s}, 2 \mathrm{H}), 7.32(\mathrm{t}, J=7.4 \mathrm{~Hz}, 1 \mathrm{H}), 7.20-7.18(\mathrm{~m}, 2 \mathrm{H}), 7.14-7.12$ $(\mathrm{m}, 2 \mathrm{H}), 6.31(\mathrm{~s}, 2 \mathrm{H}), 3.91(\mathrm{~s}, 3 \mathrm{H}), 3.62(\mathrm{~s}, 6 \mathrm{H}), 2.41(\mathrm{~s}, 3 \mathrm{H}), 1.31(\mathrm{~s}$, $18 \mathrm{H}), 1.29(\mathrm{~s}, 18 \mathrm{H})$

${ }^{13}$ C NMR $(\delta, \mathrm{ppm}) \quad 163.4,159.6,149.5(\mathrm{~d}, J=6.2 \mathrm{~Hz}), 146.6,139.7(\mathrm{~d}, J=6.2 \mathrm{~Hz}), 139.4$ $\left(75 \mathrm{MHz}, \mathrm{CD}_{2} \mathrm{Cl}_{2}\right) \quad(\mathrm{d}, J=27.3 \mathrm{~Hz}), 134.4,134.3(\mathrm{~d}, J=11.7 \mathrm{~Hz}), 131.1(\mathrm{~d}, J=3.7 \mathrm{~Hz})$, $128.6(\mathrm{~d}, J=9.1 \mathrm{~Hz}), 125.9,124.7,121.1,118.9(\mathrm{~d}, J=12.2 \mathrm{~Hz})$, $109.1(\mathrm{~d}, J=10.7 \mathrm{~Hz}), 91.4,56.3,56.2,35.4,35.1,31.6,30.5,2.8$ The peak corresponding to the carbon atom linked to the phosphorus atom couldn't be observed because of its low intensity

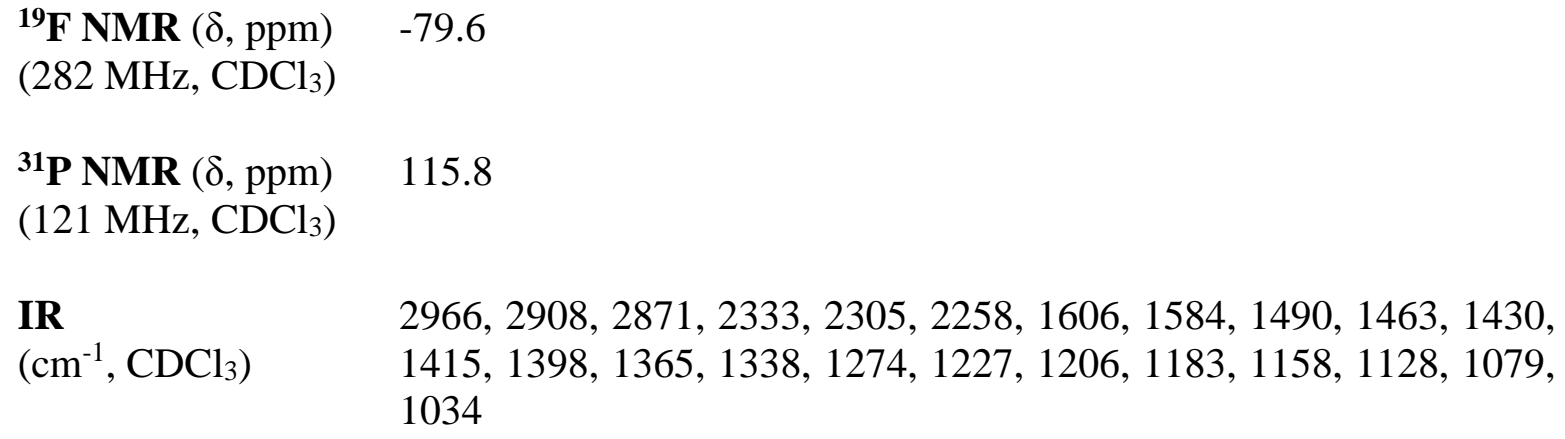

\section{Preparation of starting materials}

\section{Procedure A :}
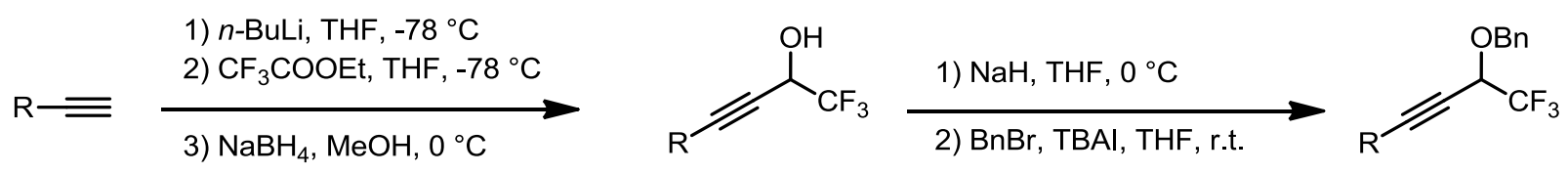

Procedure B :
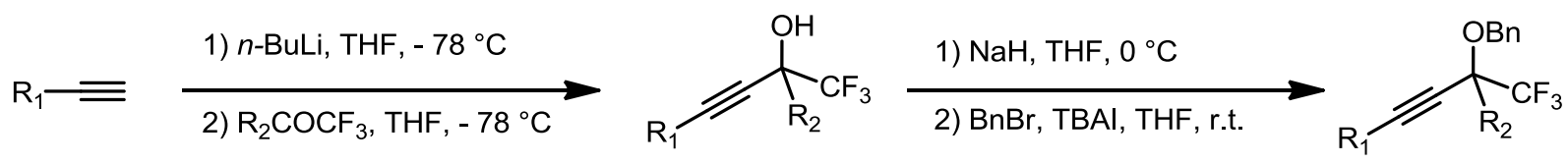

\section{Procedure C :}




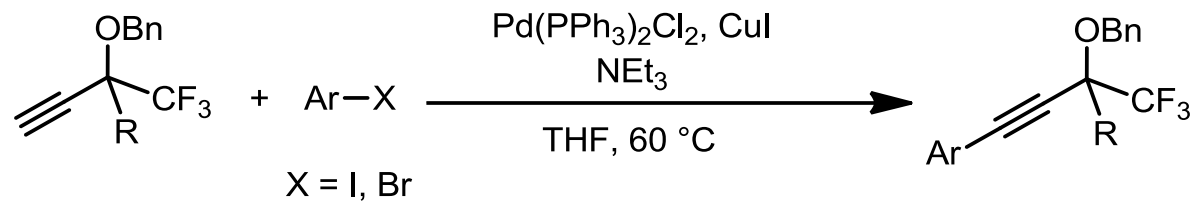

Procedure D

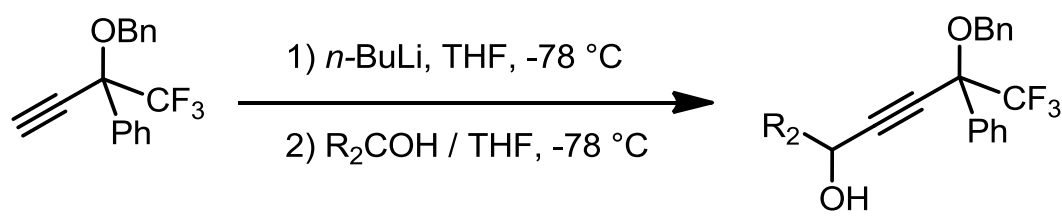

General procedure A for the preparation of $\alpha$-trifluoromethyl secondary propargylic benzyl ethers

To a solution of alkyne (1.0 eq) in THF (0.5 M) was added dropwise $n$-BuLi (1.1 eq) at $-78{ }^{\circ} \mathrm{C}$. The mixture was stirred for $1 \mathrm{~h}$ at $-78{ }^{\circ} \mathrm{C}$ and ethyl trifluoroacetate $(1.5 \mathrm{eq}$ ) was added. After the complete consumption of the alkyne (TLC), the mixture was diluted with $\mathrm{MeOH}$ (same volume than THF). The mixture was allowed to warm up to $0{ }^{\circ} \mathrm{C}$ and $\mathrm{NaBH}_{4}(1.0 \mathrm{eq})$ was added. The mixture was stirred overnight while warming up to room temperature. The reaction was then quenched with a saturated solution of $\mathrm{NH}_{4} \mathrm{Cl}$, extracted with $\mathrm{Et}_{2} \mathrm{O}(\mathrm{x} 3)$, washed with water (x3) and brine, dried over $\mathrm{MgSO}_{4}$ and concentrated under reduced pressure to give the $\alpha$-trifluoromethyl secondary propargylic alcohol. If necessary, the crude alcohol was purified by flash column chromatography.

To a solution of the alcohol dissolved in THF $(0.5 \mathrm{M})$ was added $\mathrm{NaH}(1.1 \mathrm{eq})$ at $0{ }^{\circ} \mathrm{C}$. Upon the end of the $\mathrm{H}_{2}$ formation, benzyl bromide (1.2 eq) and TBAI (0.05 eq) were added. The mixture was stirred overnight while warming up to room temperature. The reaction was quenched with a saturated solution of $\mathrm{NH}_{4} \mathrm{Cl}$, extracted with $\mathrm{Et}_{2} \mathrm{O}$ (x3), washed with brine, dried over $\mathrm{MgSO}_{4}$ and concentrated under reduced pressure. Purification by flash chromatography gave the pure $\alpha$-trifluoromethyl secondary propargylic benzyl ether.

\section{General procedure B for the preparation of $\alpha$-trifluoromethyl tertiary propargylic benzyl} ethers

To a solution of alkyne (1.0 eq) in THF (0.5 M) was added dropwise $n$-BuLi (1.1 eq) at $-78{ }^{\circ} \mathrm{C}$. The mixture was stirred for $1 \mathrm{~h}$ at $-78{ }^{\circ} \mathrm{C}$ and the trifluoromethyl ketone (1.0 eq) was added. After the complete consumption of the alkyne (TLC), the mixture was quenched with a 
saturated solution of $\mathrm{NH}_{4} \mathrm{Cl}$, extracted with $\mathrm{Et}_{2} \mathrm{O}$ (x3), washed with brine, dried over $\mathrm{MgSO}_{4}$ and concentrated under reduced pressure. Purification by flash column chromatography gave the pure $\alpha$-trifluoromethyl tertiary propargylic alcohol.

To a solution of the resulting alcohol (1.0 eq) in THF (0.5 M) was added NaH (1.1 eq) at $0{ }^{\circ} \mathrm{C}$. Upon the end of the $\mathrm{H}_{2}$ formation, benzyl bromide (1.2 eq) and TBAI (0.05 eq) were added. The mixture was stirred overnight while warming up to room temperature. The reaction was then quenched with a saturated solution of $\mathrm{NH}_{4} \mathrm{Cl}$, extracted with $\mathrm{Et}_{2} \mathrm{O}(\mathrm{x} 3)$, washed with brine, dried over $\mathrm{MgSO}_{4}$ and concentrated under reduced pressure. Purification by flash column chromatography gave the pure $\alpha$-trifluoromethyl tertiary propargylic benzyl ethers.

\section{General procedure $\mathrm{C}$ for the Sonogashira reaction of aromatic halides with alkynes}

To a solution of the aromatic halide (1.0 eq) and of the terminal alkyne (1.0 eq) in THF $(0.5 \mathrm{M})$ were added copper(I) iodide (0.05 eq) and bis(triphenylphosphine)palladium(II) chloride $(0.05 \mathrm{eq})$. Then triethylamine $(5.0 \mathrm{eq})$ was added and the mixture was heated to $60{ }^{\circ} \mathrm{C}$. Upon reaction completion (TLC), the mixture was quenched with a saturated solution of $\mathrm{NH}_{4} \mathrm{Cl}$, extracted with $\mathrm{Et}_{2} \mathrm{O}$ (x3), washed with brine, dried over $\mathrm{MgSO}_{4}$ and concentrated under reduced pressure. Purification by flash column chromatography gave the pure product.

\section{General procedure D for the synthesis of propargylic alcohols-containing substrates}

To a solution of (2-(benzyloxy)-1,1,1-trifluorobut-3-yn-2-yl)benzene (1.0 eq) in $\operatorname{THF}(0.5 \mathrm{M})$ was added dropwise $n$-BuLi (1.05 eq) at $-78{ }^{\circ} \mathrm{C}$. The mixture was stirred for $20 \mathrm{~min}$ at $-78{ }^{\circ} \mathrm{C}$ and the carbonyl compound was added. After the complete conversion of the alkyne (TLC), the mixture was allowed to warm up at room temperature. The reaction was quenched with a saturated solution of $\mathrm{NH}_{4} \mathrm{Cl}$, extracted with $\mathrm{Et}_{2} \mathrm{O}(\mathrm{x} 3)$, washed with water and brine, dried over $\mathrm{MgSO}_{4}$, and concentrated under reduced pressure. Purification by flash column chromatography afforded the pure $\alpha$-trifluoromethyl tertiary propargylic benzyl ethers.

(((1,1,1-trifluoronon-3-yn-2-yl)oxy)methyl)benzene (2a)

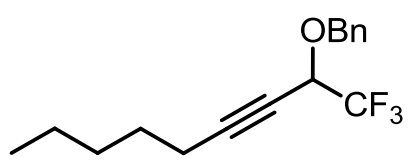

$$
\begin{gathered}
\mathrm{C}_{16} \mathrm{H}_{19} \mathrm{~F}_{3} \mathrm{O} \\
\mathrm{M}=284.32 \mathrm{~g} \cdot \mathrm{mol}^{-1}
\end{gathered}
$$


Following procedure A starting with $n$-heptyne

Flash chromatography : pure $\mathrm{PE}$ to $\mathrm{PE} / \mathrm{Et}_{2} \mathrm{O} 95$ : 5.

Overall yield: $1.78 \mathrm{~g}(64 \%)$ of a colorless oil.

${ }^{1} \mathbf{H}$ NMR $(\delta, \mathrm{ppm}) \quad 7.40-7.36(\mathrm{~m}, 5 \mathrm{H}), 4.86(\mathrm{~d}, J=11.9 \mathrm{~Hz}, 1 \mathrm{H}), 4.69(\mathrm{~d}, J=11.9 \mathrm{~Hz}$,

$\left.\left(400 \mathrm{MHz}, \mathrm{CDCl}_{3}\right) \quad 1 \mathrm{H}\right), 4.48(\mathrm{qt}, J=5.9,2.1 \mathrm{~Hz}, 1 \mathrm{H}), 2.29(\mathrm{td}, J=7.1,2.1 \mathrm{~Hz}, 2 \mathrm{H}), 1.62$ $-1.54(\mathrm{~m}, 2 \mathrm{H}), 1.50-1.31(\mathrm{~m}, 4 \mathrm{H}), 0.93(\mathrm{t}, J=7.1 \mathrm{~Hz}, 3 \mathrm{H})$

${ }^{13} \mathbf{C ~ N M R}(\delta, \mathrm{ppm}) \quad 136.4,128.7,128.4,128.3,122.8(\mathrm{q}, J=281.1 \mathrm{~Hz}), 90.6,71.0,70.5$

$\left(101 \mathrm{MHz}, \mathrm{CDCl}_{3}\right) \quad(\mathrm{q}, J=2.3 \mathrm{~Hz}), 67.7(\mathrm{q}, J=35.0 \mathrm{~Hz}), 31.1,28.0,22.3,18.8,14.1$

${ }^{19} \mathbf{F}$ NMR $(\delta, \mathrm{ppm}) \quad-78.0$

$\left(282 \mathrm{MHz}, \mathrm{CDCl}_{3}\right)$

IR

$2960,2933,2847,2238,1456,1275,1185,1158,1140,1090$

$\left(\mathrm{cm}^{-1}, \mathrm{CCl}_{4}\right)$

$\begin{array}{lll}\text { MS } & \text { Calcd for } \mathrm{C}_{16} \mathrm{H}_{19} \mathrm{~F}_{3} \mathrm{O}: 284.1388 & \text { Found : } 284.1388\end{array}$

(HRMS EI)

(6-(benzyloxy)-7,7,7-trifluorohept-4-ynyloxy)triisopropylsilane (2b)

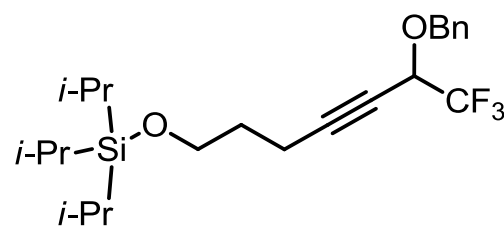

$$
\mathrm{C}_{23} \mathrm{H}_{35} \mathrm{~F}_{3} \mathrm{O}_{2} \mathrm{Si}
$$

$\mathrm{M}=428.60 \mathrm{~g} \cdot \mathrm{mol}^{-1}$

Following procedure A starting with triisopropyl(pent-4-ynyloxy)silane (5.43 mmol).

Flash chromatography : $\mathrm{PE} / \mathrm{Et}_{2} \mathrm{O} 50: 1$

Overall yield : $1.52 \mathrm{~g}(65 \%)$ of a yellow oil.

${ }^{1} \mathbf{H}$ NMR $(\delta, \mathrm{ppm}) \quad 7.78-7.09(\mathrm{~m}, 5 \mathrm{H}), 4.84(\mathrm{~d}, J=11.9 \mathrm{~Hz}, 1 \mathrm{H}), 4.67(\mathrm{~d}, J=11.9 \mathrm{~Hz}$,

$\left.\left(400 \mathrm{MHz}, \mathrm{CDCl}_{3}\right) \quad 1 \mathrm{H}\right), 4.48-4.44(\mathrm{qt}, J=5.9,2.1 \mathrm{~Hz}, 1 \mathrm{H}), 3.78(\mathrm{t}, J=6.0 \mathrm{~Hz}, 2 \mathrm{H})$, $2.42(\mathrm{td}, J=7.1,2.0 \mathrm{~Hz}, 2 \mathrm{H}), 1.78(\mathrm{tt}, J=7.1,6.0 \mathrm{~Hz}, 2 \mathrm{H}), 1.19-0.84$ $(\mathrm{m}, 21 \mathrm{H})$

${ }^{13}$ C NMR $(\delta, \mathrm{ppm}) \quad 136.4,128.7,128.4,128.3,122.8(\mathrm{q}, J=280.0 \mathrm{~Hz}), 90.3,71.0,70.5$

$\left(101 \mathrm{MHz}, \mathrm{CDCl}_{3}\right) \quad(\mathrm{q}, J=2.4 \mathrm{~Hz}), 67.7(\mathrm{q}, J=35.0 \mathrm{~Hz}), 61.7,31.6,18.1,15.3,12.1$

${ }^{19} \mathbf{F} \mathbf{N M R}(\delta, \mathrm{ppm}) \quad-78.0$

$\left(282 \mathrm{MHz}, \mathrm{CDCl}_{3}\right)$

IR

$2945,2893,2867,1463,1245,1185,1158,1140,1109,883$ 
$\left(\mathrm{cm}^{-1}, \mathrm{CCl}_{4}\right)$

MS

Calcd for $\left[M-\mathrm{C}_{3} \mathrm{H}_{7}\right]^{+}: \mathrm{C}_{20} \mathrm{H}_{28} \mathrm{~F}_{3} \mathrm{O}_{2} \mathrm{Si} 385.1811$ Found : 385.1802

(HRMS EI)

2-(5-(benzyloxy)-6,6,6-trifluorohex-3-ynyloxy)tetrahydro-2H-pyran<smiles>FC(F)(F)C(C#CCCOC1CCCCO1)C(F)(F)F</smiles>

$\mathrm{C}_{18} \mathrm{H}_{21} \mathrm{~F}_{3} \mathrm{O}_{3}$

$\mathrm{M}=342.35 \mathrm{~g} \cdot \mathrm{mol}^{-1}$

Following procedure A starting with 2-(but-3-ynyloxy)tetrahydro-2H-pyran (20.00 mmol)

Flash chromatography : PE / $\mathrm{Et}_{2} \mathrm{O} 20: 1$

Overall yield : $3.24 \mathrm{~g}(47 \%)$ of a yellowish oil obtained as a mixture of diastereomers.

${ }^{1} \mathbf{H}$ NMR $(\delta, \mathrm{ppm}) \quad 7.38-7.31(\mathrm{~m}, 5 \mathrm{H}), 4.85(\mathrm{~d}, J=11.9 \mathrm{~Hz}, 1 \mathrm{H}), 4.67(\mathrm{~d}, J=11.9 \mathrm{~Hz}$, $\left.\left(400 \mathrm{MHz}, \mathrm{CDCl}_{3}\right) \quad 1 \mathrm{H}\right), 4.66(\mathrm{t}, J=3.4 \mathrm{~Hz}, 1 \mathrm{H}), 4.49-4.44(\mathrm{~m}, 1 \mathrm{H}), 3.91-3.84 *(\mathrm{~m}$, $2 \mathrm{H}), 3.61-3.49 *(\mathrm{~m}, 2 \mathrm{H}), 2.59(\mathrm{td}, J=6.9,2.1 \mathrm{~Hz}, 2 \mathrm{H}), 1.85-1.79$ $(\mathrm{m}, 1 \mathrm{H}), 1.75-1.68(\mathrm{~m}, 1 \mathrm{H}), 1.63-1.49(\mathrm{~m}, 4 \mathrm{H})$

${ }^{13}$ C NMR $(\delta, \mathrm{ppm}) \quad 136.3,128.7,128.4,128.3,122.7(\mathrm{q}, J=281.1 \mathrm{~Hz}), 99.1 *, 87.6,71.4$ $\left(101 \mathrm{MHz}, \mathrm{CDCl}_{3}\right) \quad(\mathrm{t}, J=2.4 \mathrm{~Hz}), 71.0,67.6(\mathrm{q}, J=35.1 \mathrm{~Hz}), 65.2 *, 62.2,30.6,25.5$, $20.4,19.4$

${ }^{19}$ F NMR $(\delta, \mathrm{ppm}) \quad-77.9$

$\left(282 \mathrm{MHz}, \mathrm{CDCl}_{3}\right)$

IR 2945, 2875, 1455, 1353, 1274, 1185, 1159, 1139, 1081, 1036, 971,

$\left(\mathrm{cm}^{-1}, \mathrm{CCl}_{4}\right)$ 909,871

MS Calcd for $\mathrm{C}_{18} \mathrm{H}_{21} \mathrm{~F}_{3} \mathrm{O}_{3}: 342.1443$

Found : 342.1486

(HRMS EI)

* Observed as doublets due to the slight chemical shift difference between the two diastereomers.

\section{5-(benzyloxy)-6,6,6-trifluorohex-3-yn-1-ol}<smiles>OCCC#CC(OCc1ccccc1)C(F)(F)F</smiles>

$$
\begin{gathered}
\mathrm{C}_{13} \mathrm{H}_{13} \mathrm{~F}_{3} \mathrm{O}_{2} \\
\mathrm{M}=258.24 \mathrm{~g} \cdot \mathrm{mol}^{-1}
\end{gathered}
$$


To a solution of 2-(5-(benzyloxy)-6,6,6-trifluorohex-3-ynyloxy)tetrahydro-2H-pyran (3.50 mmol) in methanol $(0.3 \mathrm{M})$ was added $\mathrm{TsOH}(0.1 \mathrm{eq})$. The reaction was stirred overnight at room temperature and then quenched with water, extracted with $\mathrm{Et}_{2} \mathrm{O}(\mathrm{x} 3)$, washed water (x3) and brine, dried over $\mathrm{MgSO}_{4}$ and concentrated under reduced pressure. Purification by flash chromatography $\left(\mathrm{PE} / \mathrm{Et}_{2} \mathrm{O} 5: 1\right)$ gave the pure product.

Yield : $770 \mathrm{mg}(85 \%)$ of a yellow oil.

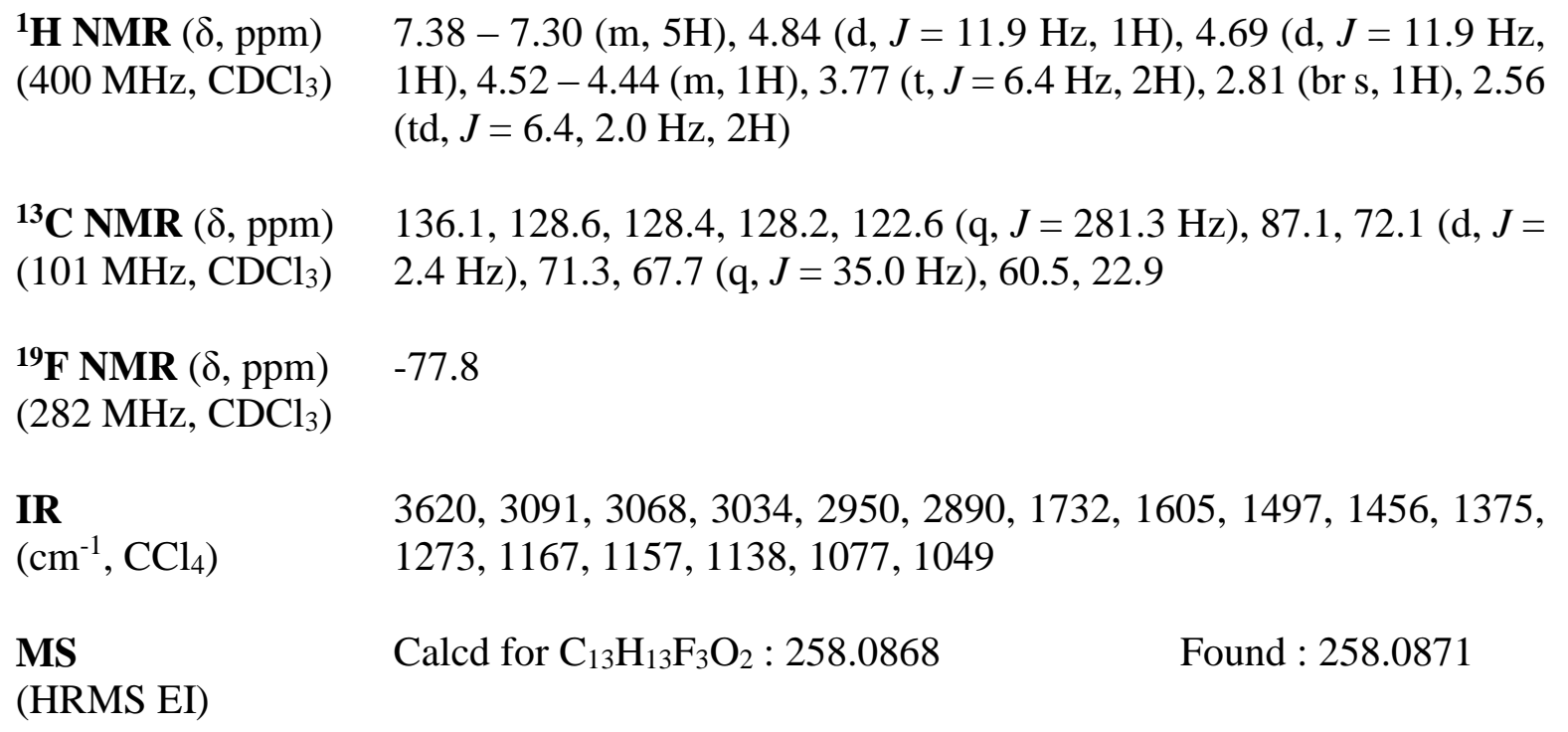

\section{5-(benzyloxy)-6,6,6-trifluorohex-3-ynyl benzoate (2c)}<smiles>O=C(OCCC#CC(OCc1ccccc1)C(F)(F)F)c1ccccc1</smiles>

$$
\begin{gathered}
\mathrm{C}_{20} \mathrm{H}_{17} \mathrm{~F}_{3} \mathrm{O}_{3} \\
\mathrm{M}=362.34 \mathrm{~g} \cdot \mathrm{mol}^{-1}
\end{gathered}
$$

To a solution of 5-(benzyloxy)-6,6,6-trifluorohex-3-yn-1-ol (1.00 mmol) in DCM (1 M) were added DMAP (0.02 eq), triethylamine (1.2 eq) and benzoyl chloride (1.1 eq). The reaction was stirred for $1 \mathrm{~h}$ at room temperature and then quenched with a saturated solution of $\mathrm{NH}_{4} \mathrm{Cl}$, extracted with $\mathrm{Et}_{2} \mathrm{O}(\mathrm{x} 3)$, washed brine, dried over $\mathrm{MgSO}_{4}$ and concentrated under reduced pressure. Purification by flash chromatography $\left(\mathrm{PE} / \mathrm{Et}_{2} \mathrm{O} 20: 1\right)$ gave the pure product. Yield : $348 \mathrm{mg}$ (95\%) of a colorless oil.

${ }^{1} \mathbf{H}$ NMR $(\delta, \mathrm{ppm}) \quad 8.08(\mathrm{dd}, J=8.3,1.4 \mathrm{~Hz}, 2 \mathrm{H}), 7.61-7.55(\mathrm{~m}, 1 \mathrm{H}), 7.44(\mathrm{t}, J=7.8 \mathrm{~Hz}$, $\left.\left(400 \mathrm{MHz}, \mathrm{CDCl}_{3}\right) \quad 2 \mathrm{H}\right), 7.37-7.31(\mathrm{~m}, 5 \mathrm{H}), 4.84(\mathrm{~d}, J=11.9 \mathrm{~Hz}, 1 \mathrm{H}), 4.66(\mathrm{~d}, J=11.9$ $\mathrm{Hz}, 1 \mathrm{H}), 4.52-4.46(\mathrm{~m}, 1 \mathrm{H}), 4.48(\mathrm{t}, J=6.7 \mathrm{~Hz}, 2 \mathrm{H}), 2.79(\mathrm{td}, J=$ 6.6, $2.0 \mathrm{~Hz}, 2 \mathrm{H}$ ) 


\begin{tabular}{|c|c|}
\hline $\begin{array}{l}{ }^{\mathbf{1 3}} \mathbf{C} \mathbf{N M R}(\delta, \mathrm{ppm}) \\
\left(101 \mathrm{MHz}, \mathrm{CDCl}_{3}\right)\end{array}$ & $\begin{array}{l}166.4,136.1,133.3,129.9,129.8,128.7,128.6,128.4,128.3,122.6 \\
(\mathrm{q}, J=281.2 \mathrm{~Hz}), 86.0,72.3(\mathrm{q}, J=2.3 \mathrm{~Hz}), 71.2,67.6(\mathrm{q}, J=35.3 \\
\mathrm{Hz}), 62.2,19.6\end{array}$ \\
\hline $\begin{array}{l}{ }^{19} \mathbf{F} \text { NMR }(\delta, \mathrm{ppm}) \\
\left(282 \mathrm{MHz}, \mathrm{CDCl}_{3}\right)\end{array}$ & -77.8 \\
\hline $\begin{array}{l}\text { IR } \\
\left(\mathrm{cm}^{-1}, \mathrm{CCl}_{4}\right)\end{array}$ & $\begin{array}{l}3091,3068,3035,2985,1967,1720,1603,1585,1497,1454,1376, \\
1316,1275,1187,1159,1139,1115,1097,1072,1028\end{array}$ \\
\hline $\begin{array}{l}\text { MS } \\
\text { (HRMS EI) }\end{array}$ & Calcd for $\mathrm{C}_{20} \mathrm{H}_{17} \mathrm{~F}_{3} \mathrm{O}_{3}: 362.1130$ \\
\hline
\end{tabular}

(((6,6,6-trifluorohex-3-yne-1,5-diyl)bis(oxy))bis(methylene))dibenzene (2d)

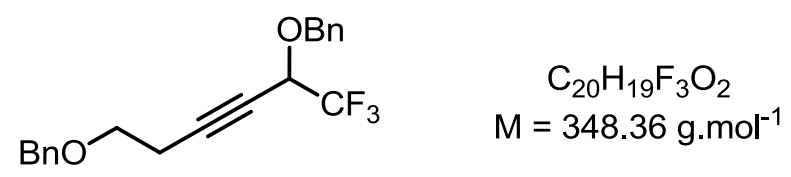

To a solution of 5-(benzyloxy)-6,6,6-trifluorohex-3-yn-1-ol (1.00 mmol) in THF (0.5 M) was added $\mathrm{NaH}$ (1.1 eq) at $0{ }^{\circ} \mathrm{C}$. Upon the end of the $\mathrm{H}_{2}$ formation, benzyl bromide (1.2 eq) and TBAI ( 0.05 eq) were added. The mixture was then stirred overnight at room temperature. The reaction was then quenched with a saturated solution of $\mathrm{NH}_{4} \mathrm{Cl}$, extracted with $\mathrm{Et}_{2} \mathrm{O}$ (x3), washed with brine, dried over $\mathrm{MgSO}_{4}$ and concentrated under reduced pressure. Purification by flash chromatography $\left(\mathrm{PE} / \mathrm{Et}_{2} \mathrm{O} 50: 1\right)$ gave the pure product.

Yield : $217 \mathrm{mg}$ (62\%) of a colorless oil.

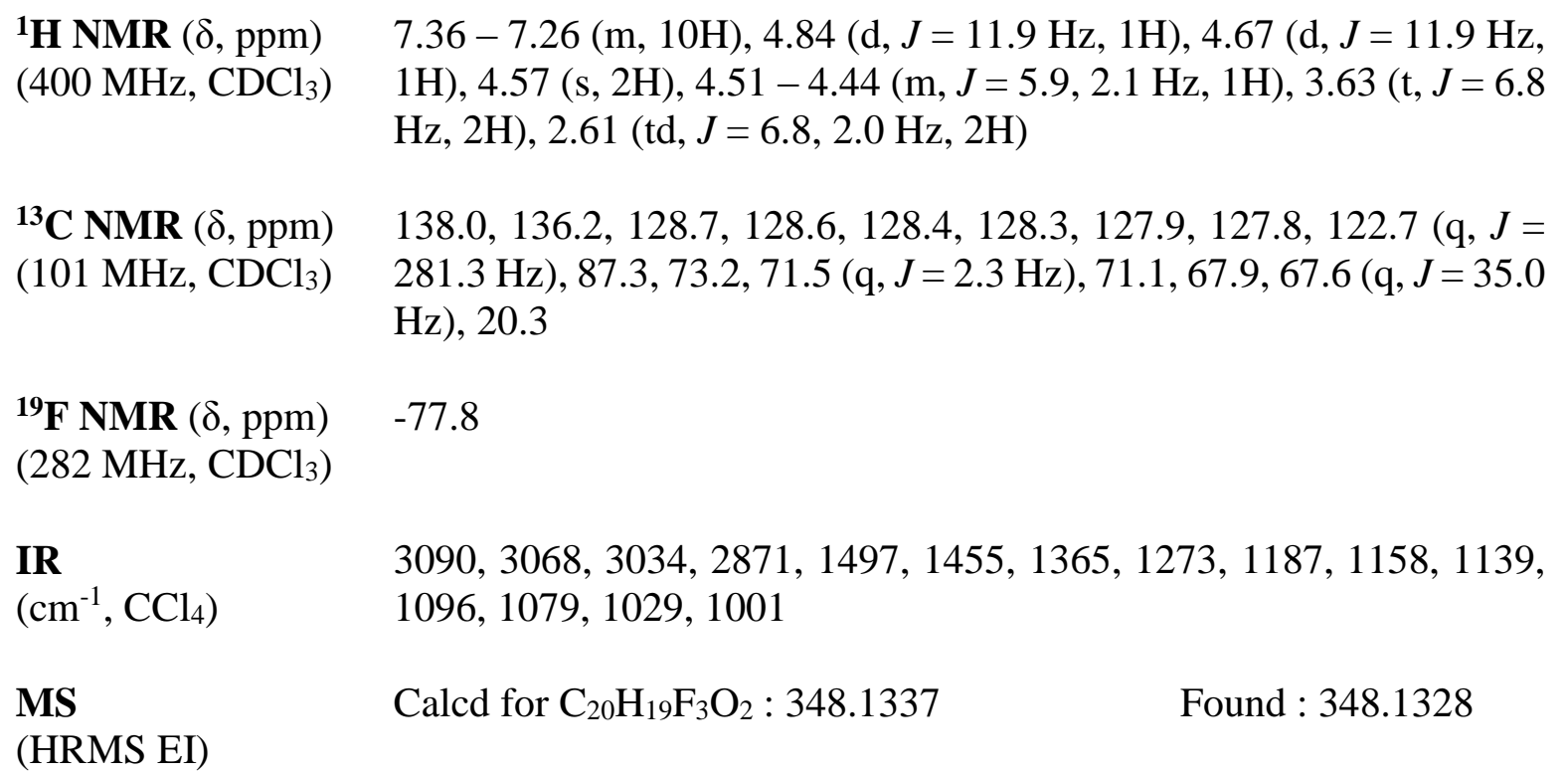




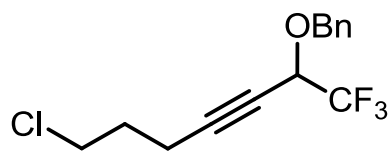

$$
\begin{gathered}
\mathrm{C}_{14} \mathrm{H}_{14} \mathrm{ClF}_{3} \mathrm{O} \\
M=290.71 \mathrm{~g} \cdot \mathrm{mol}^{-1}
\end{gathered}
$$

Following procedure A starting with 5-chloropent-1-yne $(10.00 \mathrm{mmol})$.

Flash chromatography : $\mathrm{PE} / \mathrm{Et}_{2} \mathrm{O} 50: 1$

Overall yield : $1.53 \mathrm{~g}(53 \%)$ of a colorless oil.

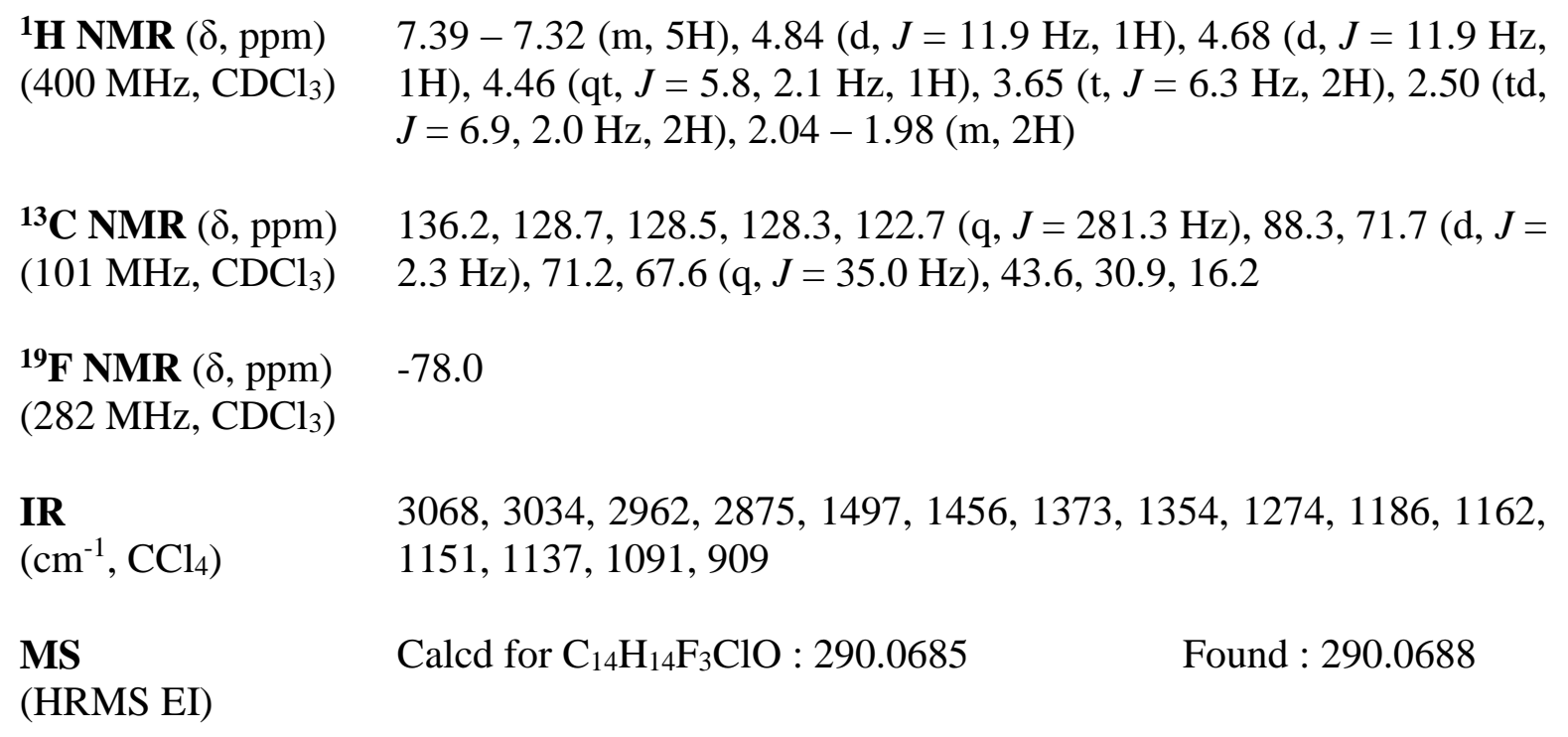

2-(6-(benzyloxy)-7,7,7-trifluorohept-4-ynyl)isoindoline-1,3-dione (2e)<smiles>O=C1c2ccccc2C(=O)N1CCCC#CC(OCc1ccccc1)C(F)(F)F</smiles>

$$
\begin{gathered}
\mathrm{C}_{22} \mathrm{H}_{18} \mathrm{~F}_{3} \mathrm{NO}_{3} \\
\mathrm{M}=401.38 \mathrm{~g} \cdot \mathrm{mol}^{-1}
\end{gathered}
$$

To a solution of ((7-chloro-1,1,1-trifluorohept-3-yn-2-yloxy)methyl)benzene $(1.00 \mathrm{mmol})$ in DMF (1 M) were added phtalimide (1.2 eq), potassium carbonate (1.0 eq) and potassium iodide (0.01 eq). The mixture was heated up to $70{ }^{\circ} \mathrm{C}$ and stirred overnight. The reaction was then quenched with a saturated solution of $\mathrm{NH}_{4} \mathrm{Cl}$, extracted with $\mathrm{Et}_{2} \mathrm{O}(\mathrm{x} 3)$, washed water (x3) and brine, dried over $\mathrm{MgSO}_{4}$ and concentrated under reduced pressure. Purification by flash chromatography $\left(\mathrm{PE} / \mathrm{Et}_{2} \mathrm{O} 2: 1\right)$ gave the pure product.

Yield : $320 \mathrm{mg}(80 \%)$ of a white solid. 


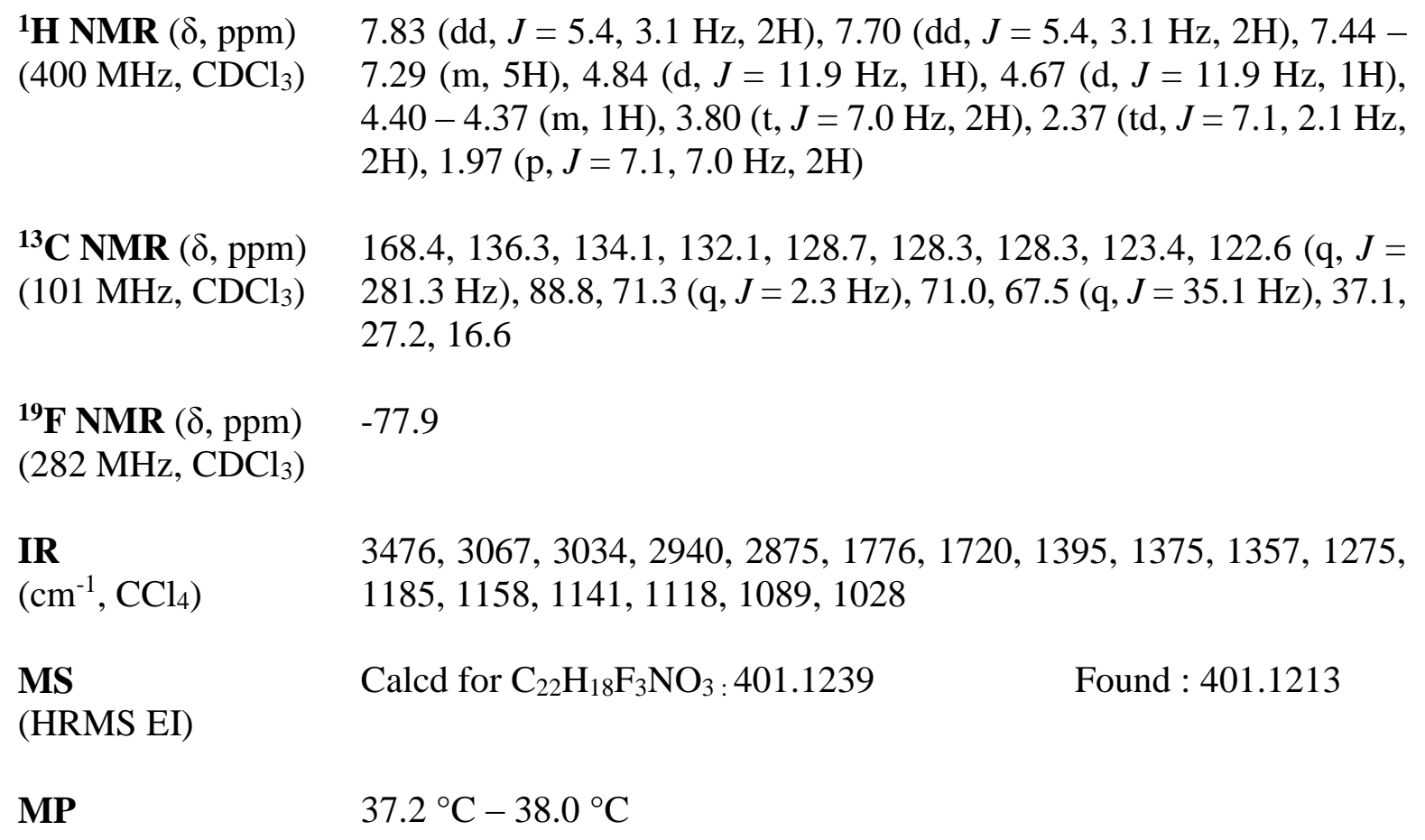

1-(3-(benzyloxy)-4,4,4-trifluorobut-1-ynyl)-4-methoxybenzene (2f)

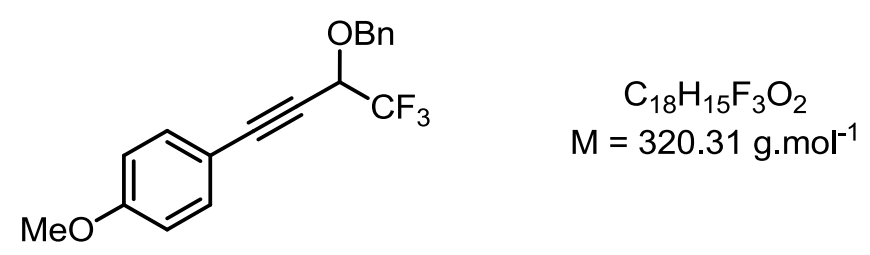

Following procedure $\mathrm{C}$ starting with $\mathbf{2 i}(0.60 \mathrm{mmol})$ and 4-iodoanisole $(0.60 \mathrm{mmol})$.

Flash chromatography : $\mathrm{PE} / \mathrm{Et}_{2} \mathrm{O} 50: 1$

Yield : $105 \mathrm{mg}(55 \%)$ of an orange oil.

${ }^{1} \mathbf{H}$ NMR $(\delta, \mathrm{ppm}) \quad 7.48-7.30(\mathrm{~m}, 7 \mathrm{H}), 6.90-6.82(\mathrm{~m}, 2 \mathrm{H}), 4.92(\mathrm{~d}, J=11.9 \mathrm{~Hz}, 1 \mathrm{H})$, $\left(400 \mathrm{MHz}, \mathrm{CDCl}_{3}\right) \quad 4.76(\mathrm{~d}, J=11.9 \mathrm{~Hz}, 1 \mathrm{H}), 4.70(\mathrm{q}, J=5.8 \mathrm{~Hz}, 1 \mathrm{H}), 3.83(\mathrm{~s}, 3 \mathrm{H})$

${ }^{13} \mathbf{C ~ N M R}(\delta, \mathrm{ppm}) \quad 160.6,136.3,133.8,128.7,128.5,128.4,122.8(\mathrm{q}, J=281.5 \mathrm{~Hz})$, $\left(101 \mathrm{MHz}, \mathrm{CDCl}_{3}\right) \quad 114.2,113.3,89.1,77.8(\mathrm{q}, J=2.2 \mathrm{~Hz}), 71.3,68.2(\mathrm{q}, J=35.2 \mathrm{~Hz})$, 55.5

${ }^{19} \mathbf{F}$ NMR $(\delta, \mathrm{ppm}) \quad-77.6$

$\left(282 \mathrm{MHz}, \mathrm{CDCl}_{3}\right)$

IR

2959, 2839, 1608, 1511, 1294, 1277, 1251, 1186, 1150, 1107, 1038, $\left(\mathrm{cm}^{-1}, \mathrm{CCl}_{4}\right) \quad 909$ 
(HRMS EI)

(3-(benzyloxy)-3-(trifluoromethyl)pent-4-yn-1-yl)benzene (2g)<smiles>C#CC(CCc1ccccc1)(OCc1ccccc1)C(F)(F)F</smiles>

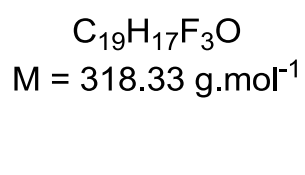

Following procedure $\mathrm{B}$ starting using a commercial solution of ethynylmagnesium bromide (1.5 eq, $0.5 \mathrm{M}$ in THF) and 1,1,1-trifluoro-4-phenyl-butan-2-one (12.0 mmol in a crude mixture prepared according to a literature procedure ${ }^{2}$ ).

Flash chromatography: pure PE to PE/EtOAc $98: 2$.

Overall yield: $2.33 \mathrm{~g}(61 \%)$ of a colorless oil.

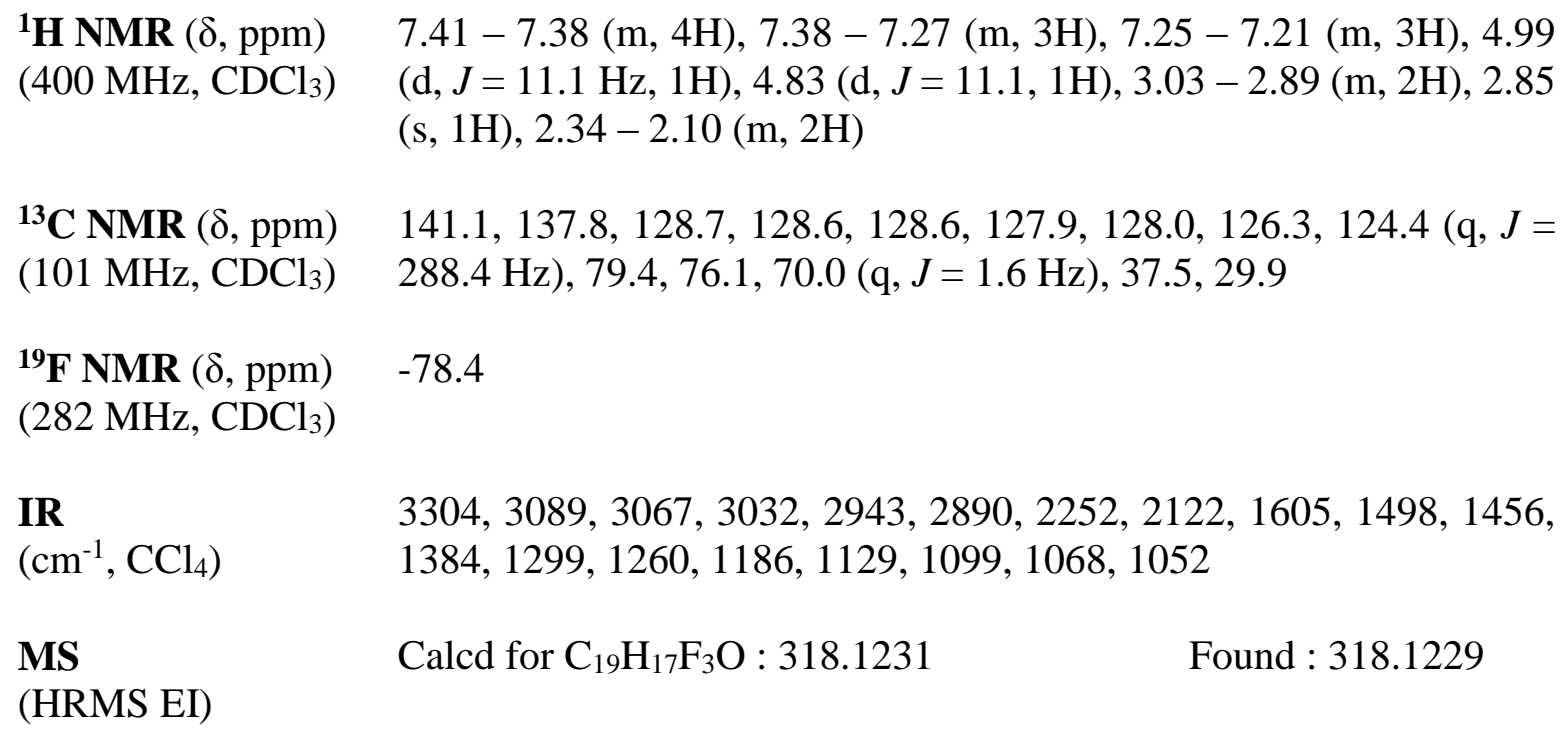

(2-(benzyloxy)-1,1,1-trifluorobut-3-yn-2-yl)benzene (2h)

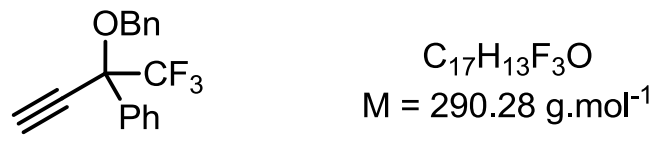

Following procedure B starting using a commercial solution of ethynylmagnesium bromide $(0.5$ $\mathrm{M}$ in THF) and 2,2,2-trifluoroacetophenone (10.00 $\mathrm{mmol})$.

Flash chromatography: pure PE to PE/EtOAc $98: 2$. 
Overall yield: $1.19 \mathrm{~g}(41 \%)$ of a pale yellow oil.

${ }^{1} \mathbf{H}$ NMR $(\delta, \mathrm{ppm}) \quad 7.82-7.80(\mathrm{~m}, 2 \mathrm{H}), 7.48-7.45(\mathrm{~m}, 3 \mathrm{H}), 7.43-7.30(\mathrm{~m}, 5 \mathrm{H}), 4.84$

$\left(400 \mathrm{MHz}, \mathrm{CDCl}_{3}\right) \quad(\mathrm{d}, J=11.1 \mathrm{~Hz}, 1 \mathrm{H}), 4.44(\mathrm{~d}, J=11.1 \mathrm{~Hz}, 1 \mathrm{H}), 2.95(\mathrm{~s}, 1 \mathrm{H})$

${ }^{13} \mathbf{C ~ N M R}(\delta, \mathrm{ppm}) \quad 137.2,133.2,130.0,128.6,128.6,128.4,128.0,127.7,122.9(\mathrm{q}, J=$ $\left.\left(101 \mathrm{MHz}, \mathrm{CDCl}_{3}\right) \quad 285.2 \mathrm{~Hz},\right), 79.6,79.5(\mathrm{q}, J=31.4 \mathrm{~Hz}), 76.5,67.8$

${ }^{19}$ F NMR $(\delta$, ppm $) \quad-79.5$

$\left(282 \mathrm{MHz}, \mathrm{CDCl}_{3}\right)$

IR

$3308,3069,3034,2936,2125,1452,1274,1191,1061$

$\left(\mathrm{cm}^{-1}, \mathrm{CCl}_{4}\right)$

MS Calcd for $\mathrm{M}: \mathrm{C}_{17} \mathrm{H}_{13} \mathrm{~F}_{3} \mathrm{O}: 290.0918 \quad$ Found : 290.0912

(HRMS EI)

((1,1,1-trifluorobut-3-yn-2-yloxy)methyl)benzene (2i)

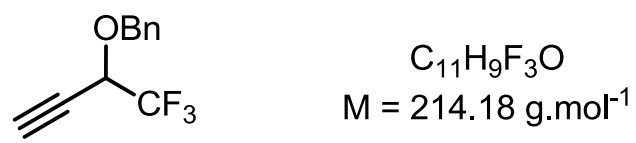

Following procedure A starting with trimethylsilylacetylene $(10.00 \mathrm{mmol})$. The trimethylsilyl group was removed during the reduction step.

Flash chromatography : $\mathrm{PE} / \mathrm{Et}_{2} \mathrm{O} 50: 1$

Overall yield : $25 \%$ of a yellowish oil. Diethyl ether used as solvent during the flash chromatography purification process could not be completely removed without a major loss of the product. Compound $\mathbf{2 i}$ was therefore kept in solution in ether and reacted as such under gold catalysis conditions (see formation of compound $4 \mathrm{i}$ ).

${ }^{1} \mathbf{H}$ NMR $(\delta, \mathrm{ppm}) \quad 7.39-7.32(\mathrm{~m}, 5 \mathrm{H}), 4.88(\mathrm{~d}, J=11.8 \mathrm{~Hz}, 1 \mathrm{H}), 4.69(\mathrm{~d}, J=11.8 \mathrm{~Hz}$, $\left.\left(400 \mathrm{MHz}, \mathrm{CDCl}_{3}\right) \quad 1 \mathrm{H}\right), 4.47(\mathrm{qd}, J=5.8,2.3 \mathrm{~Hz}, 1 \mathrm{H}), 2.64(\mathrm{~d}, J=2.2 \mathrm{~Hz}, 1 \mathrm{H})$

${ }^{13} \mathbf{C ~ N M R}(\delta, \mathrm{ppm}) \quad 135.9,128.8,128.6,128.4,122.5(\mathrm{~d}, J=281.4 \mathrm{~Hz}), 77.4,74.2(\mathrm{q}, J=$ $\left.\left(101 \mathrm{MHz}, \mathrm{CDCl}_{3}\right) \quad 2.2 \mathrm{~Hz}\right), 71.5,67.4(\mathrm{q}, J=35.5 \mathrm{~Hz})$

${ }^{19}$ F NMR $(\delta$, ppm $) \quad-77.7$

$\left(282 \mathrm{MHz}, \mathrm{CDCl}_{3}\right)$

IR $3306,3091,3069,3035,2880,2258,1497,1456,1370,1274,1190$

$\left(\mathrm{cm}^{-1}, \mathrm{CCl}_{4}\right)$ 
(HRMS EI)

(3R,10S,13R,17R)-10,13-dimethyl-12-oxo-17-((R)-6,6,6-trifluoro-5-oxohexan-2yl)hexadecahydro-1H-cyclopenta[a]phenanthren-3-yl acetate

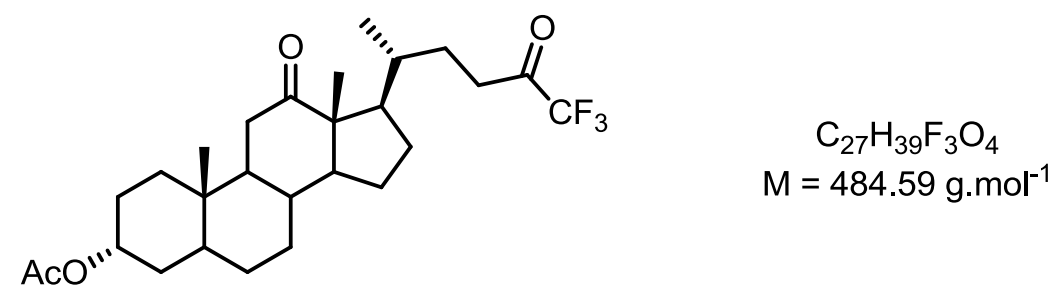

The trifluoromethyl ketone was prepared according to a literature procedure from the corresponding carboxylic acid. ${ }^{3}$ To a solution of bile acid $(1.00 \mathrm{eq}, 2.0 \mathrm{mmol}, 865.1 \mathrm{mg}$ ) in DCM (14 mL) was added oxalyl chloride (1.00 eq, $3.60 \mathrm{mmol}, 457.0 \mathrm{mg}, 0.31 \mathrm{~mL})$ at room temperature. A drop of DMF was then added and the solution was stirred for $1 \mathrm{~h}$ at room temperature. The solvent and the excess of oxalyl chloride were evaporated under a flux of nitrogen. DCM $(28 \mathrm{~mL})$ was added and the solution was cooled to $0{ }^{\circ} \mathrm{C}$. TFAA $(5.80 \mathrm{eq}, 11.60$ $\mathrm{mmol}, 2.436 \mathrm{~g}, 1.61 \mathrm{~mL}$ ) was added, followed by a dropwise addition of pyridine (7.9 eq, 26.07 $\mathrm{mmol}, 2.06 \mathrm{~g}, 2.10 \mathrm{~mL})$. The mixture was stirred for 3 hours $\left(0{ }^{\circ} \mathrm{C}\right.$ to rt $)$ and was then carefully poured onto an ice/water mixture under stirring. The layers were separated, and the aqueous phase was extracted twice with DCM. The organic phases were washed with $\mathrm{HCl}(1 \mathrm{M})$ and with brine, dried over $\mathrm{MgSO}_{4}$ and concentrated under vacuum. Precipitation from a DCM/pentane mixture allowed the isolation of the trifluoromethyl ketone as a white solid (1.044 g, $2.16 \mathrm{mmol}$, 65\% yield).

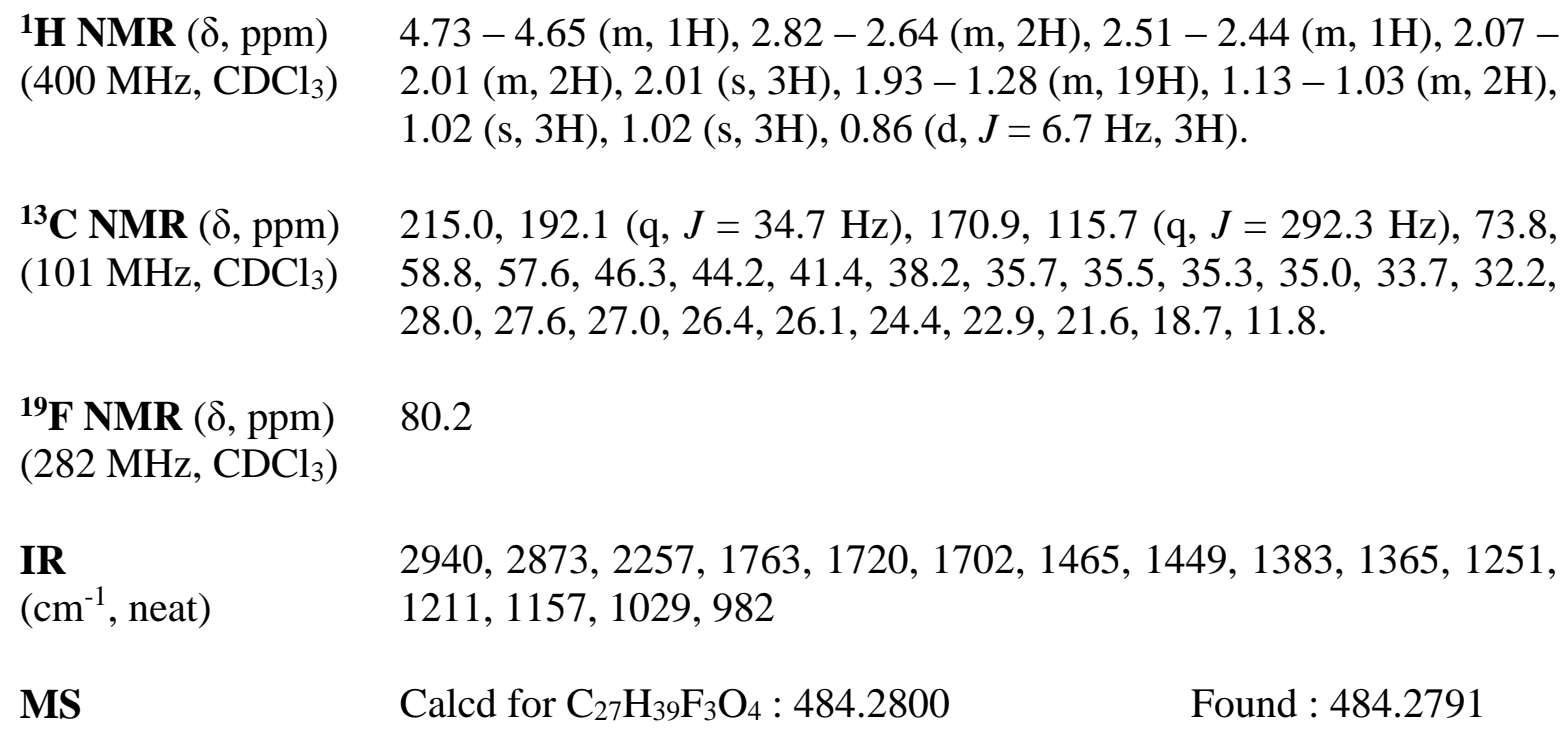


(HRMS APCI)

(3R,10S,13R,17R)-17-((2R)-5-(benzyloxy)-5-(trifluoromethyl)hept-6-yn-2-yl)-10,13-

dimethyl-12-oxohexadecahydro-1H-cyclopenta[a]phenanthren-3-yl acetate (2j)<smiles>C#C[C@](CCC[C@H](C)[C@H]1CCC2C3CCC4C[C@@H](OC(C)=O)CC[C@]4(C)C3CC(=O)[C@]21C)(OCc1ccccc1)C(C)=O</smiles>

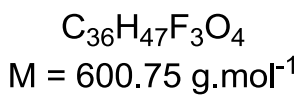

Following procedure B starting with ethynylmagnesium bromide (1.2 eq) and the synthetized trifluoromethylated ketone derived from bile acid (1.0 eq) in $\mathrm{Et}_{2} \mathrm{O}(0.1 \mathrm{M})$.

Flash chromatography : PE / EtOAc $9: 1$ to $8: 2$

Overall yield : $24 \%$ of a yellowish oil.

${ }^{1} \mathbf{H}$ NMR $(\delta, \mathrm{ppm}) \quad 7.40-7.28(\mathrm{~m}, 5 \mathrm{H}), 4.91(\mathrm{~d}, J=8.4 \mathrm{~Hz}, 1 \mathrm{H}, \mathrm{PhCHH}$ 'O, minor dia), $\left(400 \mathrm{MHz}, \mathrm{CDCl}_{3}\right) \quad 4.89\left(\mathrm{~d}, J=8.4 \mathrm{~Hz}, 1 \mathrm{H}, \mathrm{PhCH} \mathrm{H}^{\prime} \mathrm{O}\right.$, major dia $), 4.76-4.74 *(\mathrm{~m}, 1 \mathrm{H}$, $\left.\mathrm{PhCH} H^{\prime} \mathrm{O}\right), 4.72-4.67$ (m, 1H, CHOAc), 2.74 (s, 1H, minor dia), $2.73(\mathrm{~s}, 1 \mathrm{H}$, major dia), $2.50-2.44(\mathrm{~m}, 1 \mathrm{H}), 2.07-2.03(\mathrm{~m}, 2 \mathrm{H}), 2.02$ (s, 3H, $\left.\mathrm{CH}_{3}\right), 1.98-1.04(\mathrm{~m}, 23 \mathrm{H}), 1.02-1.01\left(\mathrm{~m}, 6 \mathrm{H}, \mathrm{CH}_{3} \times 2\right.$, major dia), $1.02-1.01\left(\mathrm{~m}, 3 \mathrm{H}, \mathrm{CH}_{3}\right.$, minor dia), $0.99\left(\mathrm{~s}, 3 \mathrm{H}, \mathrm{CH}_{3}\right.$, minor dia), $0.87(\mathrm{~d}, J=6.6 \mathrm{~Hz}, 3 \mathrm{H}$, major dia), $0.86(\mathrm{~d}, J=6.6 \mathrm{~Hz}, 3 \mathrm{H}$, minor dia)

${ }^{13}$ C NMR $(\delta, \mathrm{ppm}) \quad 214.9^{*}, 170.8,137.9^{*}, 128.5,127.8^{*}, 127.7,124.4(\mathrm{q}, J=288.9 \mathrm{~Hz})$, $\left(101 \mathrm{MHz}, \mathrm{CDCl}_{3}\right) \quad 79.9^{*}, 76.3^{*}, 73.9,69.7^{*}, 58.8^{*}, 57.6,46.4^{*}, 44.2,41.5,38.2,36.1^{*}$, $35.8,35.5,35.1,32.5^{*}, 32.3,28.7^{*}, 27.6^{*}, 27.1,26.5,26.2,24.4^{*}$, $22.9,21.6,19.0^{*}, 11.8$ (quadruplet for the quaternary carbon next to the $\mathrm{CF}_{3}$ group not observed due to its assumed overlapping with the $\mathrm{CDCl}_{3}$ peak)

\footnotetext{
${ }^{19}$ F NMR $(\delta, \mathrm{ppm}) \quad-78.4$ (major dia), -78.7 (minor dia)

$\left(282 \mathrm{MHz}, \mathrm{CDCl}_{3}\right)$

IR

$\left(\mathrm{cm}^{-1}\right.$, neat $)$

MS

(HRMS APCI)

$3305,3034,2941,2873,2251,2122,1719,1702,1602,1498,1465$, $1454,1383,1365,1316,1250,1182,1122,1066,1029,981$

Calcd for $\mathrm{C}_{36} \mathrm{H}_{47} \mathrm{~F}_{3} \mathrm{O}_{4}: 600.3426$

Found : 600.3423
}

* Observed as doublets due to the slight chemical shift difference between the two diastereomers. 
<smiles>FC(F)(F)C(C#CCCCc1ccccc1)C(F)(F)F</smiles>

$$
\begin{gathered}
\mathrm{C}_{20} \mathrm{H}_{19} \mathrm{~F}_{3} \mathrm{O} \\
\mathrm{M}=332.36 \mathrm{~g} \cdot \mathrm{mol}^{-1}
\end{gathered}
$$

Following procedure A starting with 5-phenyl-1-pentyne (10.00 mmol)

Flash chromatography : pure $\mathrm{PE}$ to $\mathrm{PE} / \mathrm{Et}_{2} \mathrm{O} 95$ : 5.

Overall yield : $1.90 \mathrm{~g}(57 \%)$ of a colorless oil.

${ }^{1} \mathbf{H}$ NMR $(\delta, \mathrm{ppm}) \quad 7.41-7.29(\mathrm{~m}, 7 \mathrm{H}), 7.24-7.20(\mathrm{~m}, 3 \mathrm{H}), 4.88(\mathrm{~d}, J=11.9 \mathrm{~Hz}, 1 \mathrm{H})$, $\left(400 \mathrm{MHz}, \mathrm{CDCl}_{3}\right) \quad 4.71(\mathrm{~d}, J=11.9 \mathrm{~Hz}, 1 \mathrm{H}), 4.51(\mathrm{qt}, J=5.9,2.1 \mathrm{~Hz}, 1 \mathrm{H}), 2.78-2.74$ $(\mathrm{m}, 2 \mathrm{H}), 2.31(\mathrm{td}, J=7.0,2.0 \mathrm{~Hz}, 2 \mathrm{H}), 1.94-1.86(\mathrm{~m}, 2 \mathrm{H})$

${ }^{13}$ C NMR $(\delta, \mathrm{ppm}) \quad 141.3,136.3,128.7,128.6,128.6,128.4,128.3,126.2,122.8$ (q, $J=$ $\left.\left(101 \mathrm{MHz}, \mathrm{CDCl}_{3}\right) \quad 281.2 \mathrm{~Hz}\right), 90.0,71.1(\mathrm{q}, J=2.5 \mathrm{~Hz}), 71.1,67.7(\mathrm{q}, J=35.0 \mathrm{~Hz}), 34.8$, $30.0,18.2$

${ }^{19}$ F NMR $(\delta$, ppm $) \quad-78.0$

$\left(282 \mathrm{MHz}, \mathrm{CDCl}_{3}\right)$

IR

$\left(\mathrm{cm}^{-1}, \mathrm{CCl}_{4}\right)$

MS

(HRMS EI)
$3067,3030,2956,2865,2238,1497,1455,1274,1185,1157,1138$, 1090

Calcd for $\mathrm{M}: \mathrm{C}_{20} \mathrm{H}_{19} \mathrm{~F}_{3} \mathrm{O}: 332.1388$

Found : 332.1390

(3-(benzyloxy)-4,4,4-trifluorobut-1-ynyl)benzene (2l)<smiles>FC(F)(F)C(C#Cc1ccccc1)C(F)(F)Br</smiles>

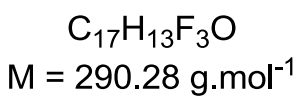

Following procedure A starting with phenylacetylene $(5.47 \mathrm{mmol})$

Flash chromatography : $\mathrm{PE} / \mathrm{Et}_{2} \mathrm{O} 50: 1$

Overall yield : $1.08 \mathrm{~g}(68 \%)$ of a yellow oil.

${ }^{1} \mathbf{H}$ NMR $(\delta, \mathrm{ppm}) \quad 7.54-7.48(\mathrm{~m}, 2 \mathrm{H}), 7.45-7.31(\mathrm{~m}, 8 \mathrm{H}), 4.94(\mathrm{~d}, J=11.9 \mathrm{~Hz}, 1 \mathrm{H})$, $\left(400 \mathrm{MHz}, \mathrm{CDCl}_{3}\right) \quad 4.77(\mathrm{~d}, J=11.9 \mathrm{~Hz}, 1 \mathrm{H}), 4.71(\mathrm{q}, J=5.8 \mathrm{~Hz}, 1 \mathrm{H})$

${ }^{13}$ C NMR $(\delta, \mathrm{ppm}) \quad 136.2,132.2,129.6,128.8,128.5,128.5,128.4,122.7(\mathrm{q}, J=281.4$ $\left.\left(101 \mathrm{MHz}, \mathrm{CDCl}_{3}\right) \quad \mathrm{Hz}\right), 121.3,89.0,79.08(\mathrm{q}, J=2.3 \mathrm{~Hz}), 71.4,68.1(\mathrm{q}, J=35.2 \mathrm{~Hz})$ 


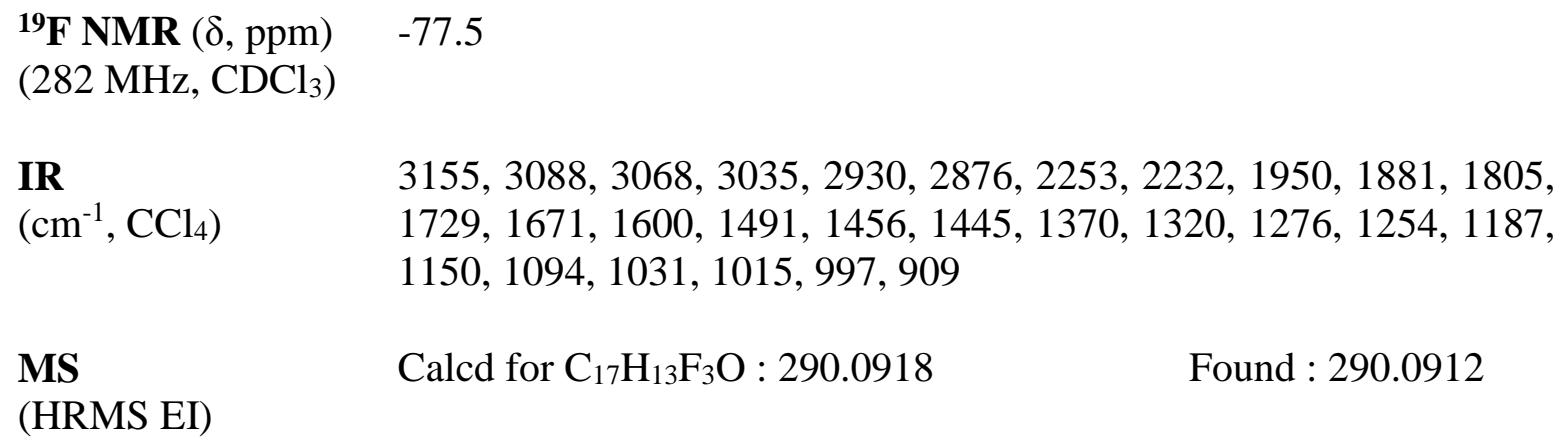<smiles>FC(F)(F)C(C#CCCCc1ccccc1)(Cc1ccccc1)C(F)(F)F</smiles>

$$
\begin{gathered}
\mathrm{C}_{21} \mathrm{H}_{19} \mathrm{~F}_{5} \mathrm{O} \\
\mathrm{M}=382.37 \mathrm{~g} \cdot \mathrm{mol}^{-1}
\end{gathered}
$$

Following procedure A starting with 5-phenylpent-1-yne $(5.00 \mathrm{mmol})$ and using ethyl pentafluoropropionate instead of ethyl trifluoroacetate.

Flash chromatography : $\mathrm{PE} / \mathrm{Et}_{2} \mathrm{O} 50: 1$

Overall yield : $1.13 \mathrm{~g} \mathrm{(59 \% )} \mathrm{of} \mathrm{a} \mathrm{yellowish} \mathrm{oil.}$

${ }^{1} \mathbf{H}$ NMR $(\delta, \mathrm{ppm}) \quad 7.27-7.23(\mathrm{~m}, 4 \mathrm{H}), 7.23-7.16(\mathrm{~m}, 3 \mathrm{H}), 7.12-7.06(\mathrm{~m}, 3 \mathrm{H}), 4.77$ $\left(400 \mathrm{MHz}, \mathrm{CDCl}_{3}\right) \quad(\mathrm{d}, J=11.8 \mathrm{~Hz}, 1 \mathrm{H}), 4.56(\mathrm{~d}, J=11.8 \mathrm{~Hz}, 1 \mathrm{H}), 4.54-4.47(\mathrm{~m}, 1 \mathrm{H})$, $2.63(\mathrm{t}, J=7.5 \mathrm{~Hz}, 2 \mathrm{H}), 2.19(\mathrm{td}, J=7.2,2.0 \mathrm{~Hz}, 2 \mathrm{H}), 1.77(\mathrm{p}, J=7.3$ $\mathrm{Hz}, 2 \mathrm{H})$

${ }^{13} \mathbf{C ~ N M R}(\delta, \mathrm{ppm}) \quad 141.3,136.2,128.7,128.6,128.6,128.4,128.2,126.2,118.9(\mathrm{qt}, J=$ $\left.\left(101 \mathrm{MHz}, \mathrm{CDCl}_{3}\right) \quad 285.0,35.1 \mathrm{~Hz}\right), 112.0(\mathrm{ddq}, J=261.7,255.8,36.2 \mathrm{~Hz}), 91.0,71.0$, $70.4,67.3(\mathrm{dd}, J=29.8,24.5 \mathrm{~Hz}), 34.7,29.9,18.2$

${ }^{19} \mathbf{F}$ NMR $(\delta, \mathrm{ppm}) \quad-82.1,-120.8(\mathrm{dd}, J=273.8,4.5 \mathrm{~Hz}),-126.8(\mathrm{dd}, J=274.1,13.8 \mathrm{~Hz})$ $\left(282 \mathrm{MHz}, \mathrm{CDCl}_{3}\right)$

IR

$\left(\mathrm{cm}^{-1}, \mathrm{CCl}_{4}\right)$

MS

(HRMS EI)
3088, 3067, 3031, 2947, 2866, 1952, 1876, 1810, 1603, 1497, 1455, $1430,1368,1315,1218,1203,1149,1139,1078,1034$

Calcd for $\mathrm{C}_{21} \mathrm{H}_{19} \mathrm{~F}_{5} \mathrm{O}: 382.1356$

Found : 382.1360 


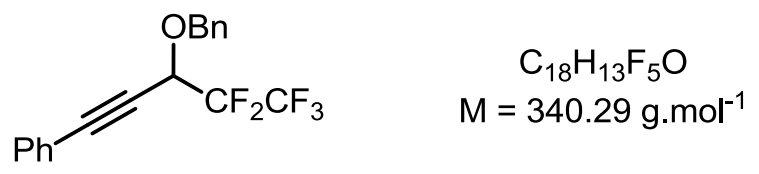

Following procedure A starting with phenylacetylene $(3.00 \mathrm{mmol})$ and using ethyl pentafluoropropionate instead of ethyl trifluoroacetate.

Flash chromatography : $\mathrm{PE} / \mathrm{Et}_{2} \mathrm{O} 50: 1$

Overall yield : $360 \mathrm{mg}(35 \%)$ of a yellow oil.

${ }^{1} \mathbf{H}$ NMR $(\delta, \mathrm{ppm}) \quad 7.57-7.49(\mathrm{~m}, 2 \mathrm{H}), 7.43-7.34(\mathrm{~m}, 8 \mathrm{H}), 4.96(\mathrm{~d}, J=11.9 \mathrm{~Hz}, 1 \mathrm{H})$, $\left(400 \mathrm{MHz}, \mathrm{CDCl}_{3}\right) \quad 4.86-4.80(\mathrm{~m}, 1 \mathrm{H}), 4.76(\mathrm{~d}, J=11.9 \mathrm{~Hz}, 1 \mathrm{H})$

${ }^{13}$ C NMR $(\delta, \mathrm{ppm}) \quad 136.1,132.3,129.6,128.7,128.6,128.5,128.4,121.4,118.9$ (qt, $J=$ $\left.\left(101 \mathrm{MHz}, \mathrm{CDCl}_{3}\right) \quad 287.1,35.0 \mathrm{~Hz}\right), 112.0(\mathrm{ddq}, J=262.2,250.4,29.7 \mathrm{~Hz}), 90.0,78.5(\mathrm{t}$, $J=2.9 \mathrm{~Hz}), 71.4,67.8(\mathrm{dd}, J=29.9,24.9 \mathrm{~Hz})$

${ }^{19}$ F NMR $(\delta, \mathrm{ppm}) \quad-82.2(\mathrm{~s}),-120.7(\mathrm{~d}, J=273.8 \mathrm{~Hz}),-126.4(\mathrm{~d}, J=274.0 \mathrm{~Hz})$

$\left(282 \mathrm{MHz}, \mathrm{CDCl}_{3}\right)$

IR $3088,3068,3035,2930,2877,2258,2238,1971,1953,1885,1808$, $\left(\mathrm{cm}^{-1}, \mathrm{CCl}_{4}\right) \quad 1599,1491,1456,1445,1368,1359,1316,1260,1219,1199,1095$, 1043

$\begin{array}{lll}\text { MS } & \text { Calcd for } \mathrm{C}_{18} \mathrm{H}_{13} \mathrm{~F}_{5} \mathrm{O}: 340.0887 & \text { Found : } 340.0883\end{array}$

(HRMS EI)

(6-(benzyloxy)-7,7-difluorohept-4-yn-1-yl)benzene (2o)

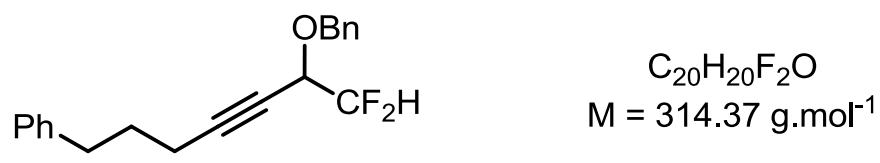

Following procedure A starting with 5-phenylpent-1-yne and using ethyl difluoroacetate instead of ethyl trifluoroacetate.

Flash chromatography : pure PE to PE/EtOAc 95 : 5.

Overall yield : $251 \mathrm{mg}$ (66\%) of a pale yellow oil.

${ }^{1} \mathbf{H}$ NMR $(\delta, \mathrm{ppm}) \quad 7.38-7.29(\mathrm{~m}, 7 \mathrm{H}), 7.22-7.18(\mathrm{~m}, 3 \mathrm{H}), 5.74(\mathrm{td}, J=55.7,4.3 \mathrm{~Hz}$, $\left.\left(400 \mathrm{MHz}, \mathrm{CDCl}_{3}\right) \quad 1 \mathrm{H}\right), 4.85(\mathrm{~d}, J=11.8 \mathrm{~Hz}, 1 \mathrm{H}), 4.63(\mathrm{~d}, J=11.8 \mathrm{~Hz}, 1 \mathrm{H}), 4.34-4.27$ 
$(\mathrm{m}, 1 \mathrm{H}), 2.75(\mathrm{t}, J=7.3 \mathrm{~Hz}, 2 \mathrm{H}), 2.30(\mathrm{td}, J=7.2,2.0 \mathrm{~Hz}, 2 \mathrm{H}), 1.88$ $(\mathrm{p}, J=7.2 \mathrm{~Hz}, 2 \mathrm{H})$
${ }^{13} \mathbf{C ~ N M R}(\delta, \mathrm{ppm}) \quad 141.4,136.8,128.7,128.7,128.6,128.3,128.3,126.1,113.8(\mathrm{t}, J=$ $\left.\left(101 \mathrm{MHz}, \mathrm{CDCl}_{3}\right) \quad 246.2 \mathrm{~Hz}\right), 89.8,72.8(\mathrm{dd}, J=6.7,3.2 \mathrm{~Hz}), 71.0,69.0(\mathrm{t}, J=27.5 \mathrm{~Hz})$, $34.8,30.1,18.3$

${ }^{19}$ F NMR $(\delta, \mathrm{ppm}) \quad-126.0(\mathrm{ddd}, J=284.2,55.8,9.6 \mathrm{~Hz}),-128.1(\mathrm{ddd}, J=284.2,55.8,9.6$ $\left.\left(282 \mathrm{MHz}, \mathrm{CDCl}_{3}\right) \quad \mathrm{Hz}\right)$

IR 3088, 3067, 3030, 2928, 2860, 2238, 1604, 1497, 1455, 1382, 1305, $\left(\mathrm{cm}^{-1}, \mathrm{CCl}_{4}\right) \quad 1264,1100,1029$

MS Calcd for $\mathrm{M}: \mathrm{C}_{20} \mathrm{H}_{20} \mathrm{~F}_{2} \mathrm{O}: 314.1482 \quad$ Found : 314.1490

(HRMS EI)

(3-(benzyloxy)-4,4,4-trifluoro-3-methylbut-1-yn-1-yl)benzene (2p)

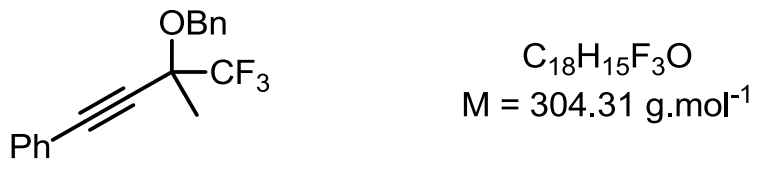

To a solution of 4-phenylbut-3-yn-2-one (1.0 equiv, $1 \mathrm{mmol}, 144.2 \mathrm{mg})$ and trifluoromethyltrimethylsilane (1.2 equiv, $1.2 \mathrm{mmol}, 170.6 \mathrm{mg}, 0.18 \mathrm{~mL})$ in THF $(0.67 \mathrm{M})$ at 0 ${ }^{\circ} \mathrm{C}$ was added a catalytic amount of TBAF ( $1 \mathrm{M}$ in THF, 0.1 equiv, $0.1 \mathrm{mmol}, 0.1 \mathrm{~mL}$ ) and the reaction was stirred for 2 hours at $0{ }^{\circ} \mathrm{C}$. Water and aqueous $\mathrm{HCl}$ were added and the solution was stirred overnight at room temperature. The mixture was extracted with $\mathrm{Et}_{2} \mathrm{O}(3 \mathrm{x})$, washed with water $(1 \mathrm{x})$, dried over $\mathrm{MgSO}_{4}$ and concentrated over reduced pressure. Purification by flash chromatography (PE/EtOAc $9: 1)$ afforded the desired alcohol as a yellow oil (166 mg, $66 \%$ yield).

To a solution of the resulting alcohol in $\operatorname{THF}(0.5 \mathrm{M})$ was added $\mathrm{NaH}\left(1.1\right.$ equiv) at $0{ }^{\circ} \mathrm{C}$. Upon the end of the $\mathrm{H}_{2}$ formation, benzyl bromide (1.2 equiv) and TBAI ( 0.05 equiv) were added. The mixture was then stirred overnight at room temperature. The reaction was then quenched with a saturated solution of $\mathrm{NH}_{4} \mathrm{Cl}$, extracted with $\mathrm{Et}_{2} \mathrm{O}$ (x3), washed with brine, dried over $\mathrm{MgSO}_{4}$ and concentrated under reduced pressure. Purification by flash chromatography (PE / AcOEt $98: 2$ ) gave the pure product.

Overall yield : $107 \mathrm{mg}(70 \%)$ of a yellow oil. 


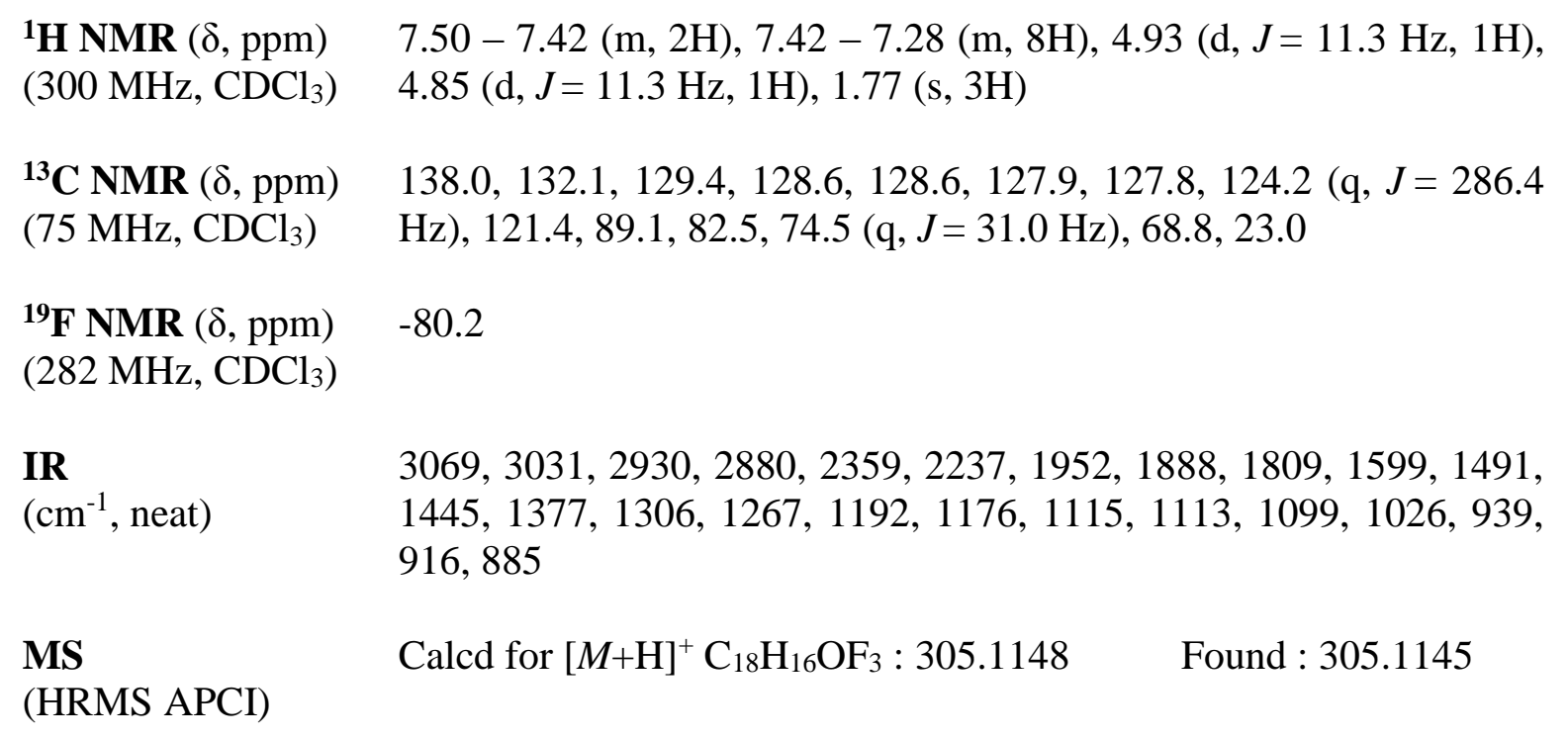

(6-(benzyloxy)-6-(trifluoromethyl)dec-4-ynyl)benzene $(2 q)^{4}$

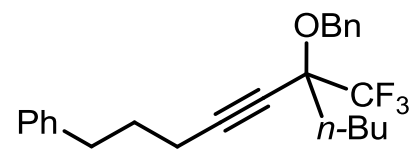

$$
\begin{gathered}
\mathrm{C}_{24} \mathrm{H}_{27} \mathrm{~F}_{3} \mathrm{O} \\
\mathrm{M}=388.47 \mathrm{~g} \cdot \mathrm{mol}^{-1}
\end{gathered}
$$

To a solution of 5-phenylpent-1-yne (2.0 eq) in THF (0.5 M) was added dropwise $n$-BuLi (2.2 eq) at $-78{ }^{\circ} \mathrm{C}$. The mixture was stirred for $1 \mathrm{~h}$ at $-78{ }^{\circ} \mathrm{C}$ and ethyl trifluoroacetate (1.0 eq) was added. After the complete consumption of the alkyne (TLC), the reaction was quenched with a saturated solution of $\mathrm{NH}_{4} \mathrm{Cl}$, extracted with $\mathrm{Et}_{2} \mathrm{O}(\mathrm{x} 3)$, washed with brine, dried over $\mathrm{MgSO}_{4}$ and concentrated under reduced pressure. Purification by flash chromatography (PE / EtOAc $50: 1$ to $10: 1)$ gave the alcohol as a yellow oil (33\% yield). The product resulting from the double addition of the alkyne on ethyl trifluoroacetate was also isolated as a yellow oil (55\% yield).

To a solution of the resulting alcohol in THF $(0.5 \mathrm{M})$ was added $\mathrm{NaH}(1.1 \mathrm{eq})$ at $0{ }^{\circ} \mathrm{C}$. Upon the end of the $\mathrm{H}_{2}$ formation, benzyl bromide (1.2 eq) and TBAI (0.05 eq) were added. The mixture was then stirred overnight at room temperature. The reaction was then quenched with a saturated solution of $\mathrm{NH}_{4} \mathrm{Cl}$, extracted with $\mathrm{Et}_{2} \mathrm{O}(\mathrm{x} 3)$, washed with brine, dried over $\mathrm{MgSO}_{4}$ and concentrated under reduced pressure. Purification by flash chromatography (PE / $\left.\mathrm{Et}_{2} \mathrm{O} 50: 1\right)$ gave the pure product.

Overall yield : $25 \%$ of a yellow oil.

${ }^{1} \mathbf{H}$ NMR $(\delta, \mathrm{ppm}) \quad 7.43-7.38(\mathrm{~m}, 4 \mathrm{H}), 7.37-7.30(\mathrm{~m}, 3 \mathrm{H}), 7.29-7.18(\mathrm{~m}, 3 \mathrm{H}), 4.96$ $\left(400 \mathrm{MHz}, \mathrm{CDCl}_{3}\right) \quad(\mathrm{d}, J=11.3 \mathrm{~Hz}, 1 \mathrm{H}), 4.79(\mathrm{~d}, J=11.3,1 \mathrm{H}), 2.79(\mathrm{t}, J=8 \mathrm{~Hz}, 2 \mathrm{H})$, 
$2.36(\mathrm{t}, J=6.9 \mathrm{~Hz}, 2 \mathrm{H}), 1.98-1.84(\mathrm{~m}, 4 \mathrm{H}), 1.75-1.60(\mathrm{~m}, 2 \mathrm{H}), 1.50$ $-1.35(\mathrm{~m}, 2 \mathrm{H}), 0.98(\mathrm{t}, J=8 \mathrm{~Hz}, 3 \mathrm{H})$

${ }^{13}$ C NMR $(\delta, \mathrm{ppm}) \quad 141.3,138.4,128.6,128.6,128.5,127.8(\mathrm{x} 2), 126.2,124.8(\mathrm{q}, J=$ $\left.\left(101 \mathrm{MHz}, \mathrm{CDCl}_{3}\right) \quad 288.0 \mathrm{~Hz}\right), 91.3,77.4(\mathrm{q}, J=29.2 \mathrm{~Hz}), 73.3,69.5,35.6,34.8,30.1$, $25.8,22.8,18.2,14.1$

${ }^{19}$ F NMR $(\delta, \mathrm{ppm}) \quad-78.7$

$\left(282 \mathrm{MHz}, \mathrm{CDCl}_{3}\right)$

IR

$3086,3035,3029,2960,2934,2868,1976,1603,1496,1455,1380$,

$\left(\mathrm{cm}^{-1}, \mathrm{CCl}_{4}\right)$ $1292,1253,1204,1156,1121,1031$

MS

Calcd for $\mathrm{C}_{24} \mathrm{H}_{27} \mathrm{~F}_{3} \mathrm{O}: 388.2014$

Found : 388.2011

(HRMS EI)

(3-(benzyloxy)-3-(trifluoromethyl)pent-1-yne-1,5-diyl)dibenzene (2r)

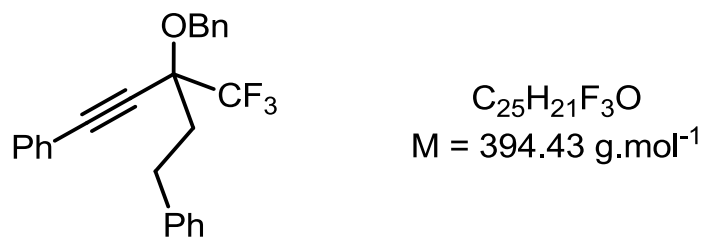

Following procedure B starting with phenylacetylene (2.5 mmol) and 1,1,1-trifluoro-4-phenylbutan-2-one.

Flash chromatography : PE / $\mathrm{Et}_{2} \mathrm{O} 100: 1$

Overall yield : $588 \mathrm{mg}(60 \%)$ of a yellowish oil.

${ }^{1} \mathbf{H}$ NMR $(\delta, \mathrm{ppm}) \quad 7.65-7.58(\mathrm{~m}, 2 \mathrm{H}), 7.52-7.41(\mathrm{~m}, 7 \mathrm{H}), 7.41-7.34(\mathrm{~m}, 3 \mathrm{H}), 7.34-$ $\left(400 \mathrm{MHz}, \mathrm{CDCl}_{3}\right) \quad 7.24(\mathrm{~m}, 3 \mathrm{H}), 5.14(\mathrm{~d}, J=11.3 \mathrm{~Hz}, 1 \mathrm{H}), 4.96(\mathrm{~d}, J=11.3 \mathrm{~Hz}, 1 \mathrm{H})$, $3.09(\mathrm{~m}, 2 \mathrm{H}), 2.37(\mathrm{~m}, 2 \mathrm{H})$

${ }^{13} \mathbf{C ~ N M R}(\delta, \mathrm{ppm}) \quad 141.3,138.1,132.2,129.6,128.7,128.6,128.6,128.6,128.0,127.9$, $\left(101 \mathrm{MHz}, \mathrm{CDCl}_{3}\right) \quad 126.3,124.6(\mathrm{q}, J=288.4 \mathrm{~Hz}), 121.3,91.1,81.2,77.6(\mathrm{q}, J=29.7 \mathrm{~Hz})$, $70.0,37.8,30.2$

${ }^{19} \mathbf{F}$ NMR $(\delta, \mathrm{ppm}) \quad-78.1$

$\left(282 \mathrm{MHz}, \mathrm{CDCl}_{3}\right)$

IR

$\left(\mathrm{cm}^{-1}, \mathrm{CCl}_{4}\right)$

MS

(HRMS EI)
$3088,3067,3032,2974,2942,2875,2255,2237,1952,1883,1808$, 1603, 1492, 1445, 1383, 1320, 1254, 1181

Calcd for $\mathrm{C}_{25} \mathrm{H}_{21} \mathrm{~F}_{3} \mathrm{O}: 394.1544$

Found : 394.1561 
<smiles>COc1cccc(C#CC(CCc2ccccc2)(OCc2ccccc2)C(F)(F)F)c1</smiles>

$$
\begin{gathered}
\mathrm{C}_{26} \mathrm{H}_{23} \mathrm{~F}_{3} \mathrm{O}_{2} \\
\mathrm{M}=424.45 \mathrm{~g} \cdot \mathrm{mol}^{-1}
\end{gathered}
$$

Following procedure $\mathrm{C}$ with $(\mathbf{2 g})(1.50 \mathrm{mmol})$ and 3 -anisole $(1.50 \mathrm{mmol})$.

Flash chromatography : $\mathrm{PE} / \mathrm{Et}_{2} \mathrm{O} 50: 1$

Yied : $540 \mathrm{mg}(85 \%)$ of a colorless oil.

${ }^{1} \mathbf{H}$ NMR $(\delta, \mathrm{ppm}) \quad 7.42-7.27(\mathrm{~m}, 8 \mathrm{H}), 7.25-7.16(\mathrm{~m}, 3 \mathrm{H}), 7.12(\mathrm{dt}, J=7.6,1.2 \mathrm{~Hz}$, $\left.\left(400 \mathrm{MHz}, \mathrm{CDCl}_{3}\right) \quad 1 \mathrm{H}\right), 7.03-7.00(\mathrm{~m}, 1 \mathrm{H}), 6.96(\mathrm{ddd}, J=8.3,2.6,1.0 \mathrm{~Hz}, 1 \mathrm{H}), 5.03(\mathrm{~d}$, $J=11.2 \mathrm{~Hz}, 1 \mathrm{H}), 4.87(\mathrm{~d}, J=11.2 \mathrm{~Hz}, 1 \mathrm{H}), 3.83(\mathrm{~s}, 3 \mathrm{H}), 3.03-2.98$ $(\mathrm{m}, 2 \mathrm{H}), 2.35-2.20(\mathrm{~m}, 2 \mathrm{H})$

${ }^{13}$ C NMR $(\delta, \mathrm{ppm}) \quad 159.7,141.3,138.1,129.7,128.7,128.6,128.6,128.0,127.9,126.3$, $\left(101 \mathrm{MHz}, \mathrm{CDCl}_{3}\right) \quad 124.8,124.6(\mathrm{q}, J=288.3 \mathrm{~Hz}), 122.3,117.1,116.1,90.9,81.00,77.7$ $(\mathrm{q}, J=29.7 \mathrm{~Hz}), 70.0,55.5,37.7,30.2$

${ }^{19}$ F NMR $(\delta, \mathrm{ppm}) \quad-78.2$

$\left(282 \mathrm{MHz}, \mathrm{CDCl}_{3}\right)$

IR $3067,3032,3009,2964,2942,2876,2838,2251,2232,1711,1604$, $\left(\mathrm{cm}^{-1}, \mathrm{CCl}_{4}\right) \quad 1577,1482,1466,1456,1322,1287,1259,1225,1183,1121,1095$, 1065,1051

MS

Calcd for $\mathrm{C}_{26} \mathrm{H}_{23} \mathrm{~F}_{3} \mathrm{O}_{2}: 424.1650$

Found : 424.1658

(HRMS EI)

1-(3-(benzyloxy)-5-phenyl-3-(trifluoromethyl)pent-1-yn-1-yl)-4-bromobenzene (2t)<smiles>FC(F)(F)C(C#Cc1ccc(Br)cc1)(CCc1ccccc1)C(F)(F)Br</smiles>

$$
\begin{gathered}
\mathrm{C}_{25} \mathrm{H}_{20} \mathrm{BrF}_{3} \mathrm{O} \\
\mathrm{M}=473.32 \mathrm{~g} \cdot \mathrm{mol}^{-1}
\end{gathered}
$$

Following procedure $\mathrm{C}$ with $(\mathbf{2 g})(1.50 \mathrm{mmol})$ and 4-bromoiodobenzene $(1.50 \mathrm{mmol})$.

Flash chromatography : PE / $\mathrm{Et}_{2} \mathrm{O} 50: 1$

Yied : $640 \mathrm{mg}$ (90\%) of a colorless oil. 


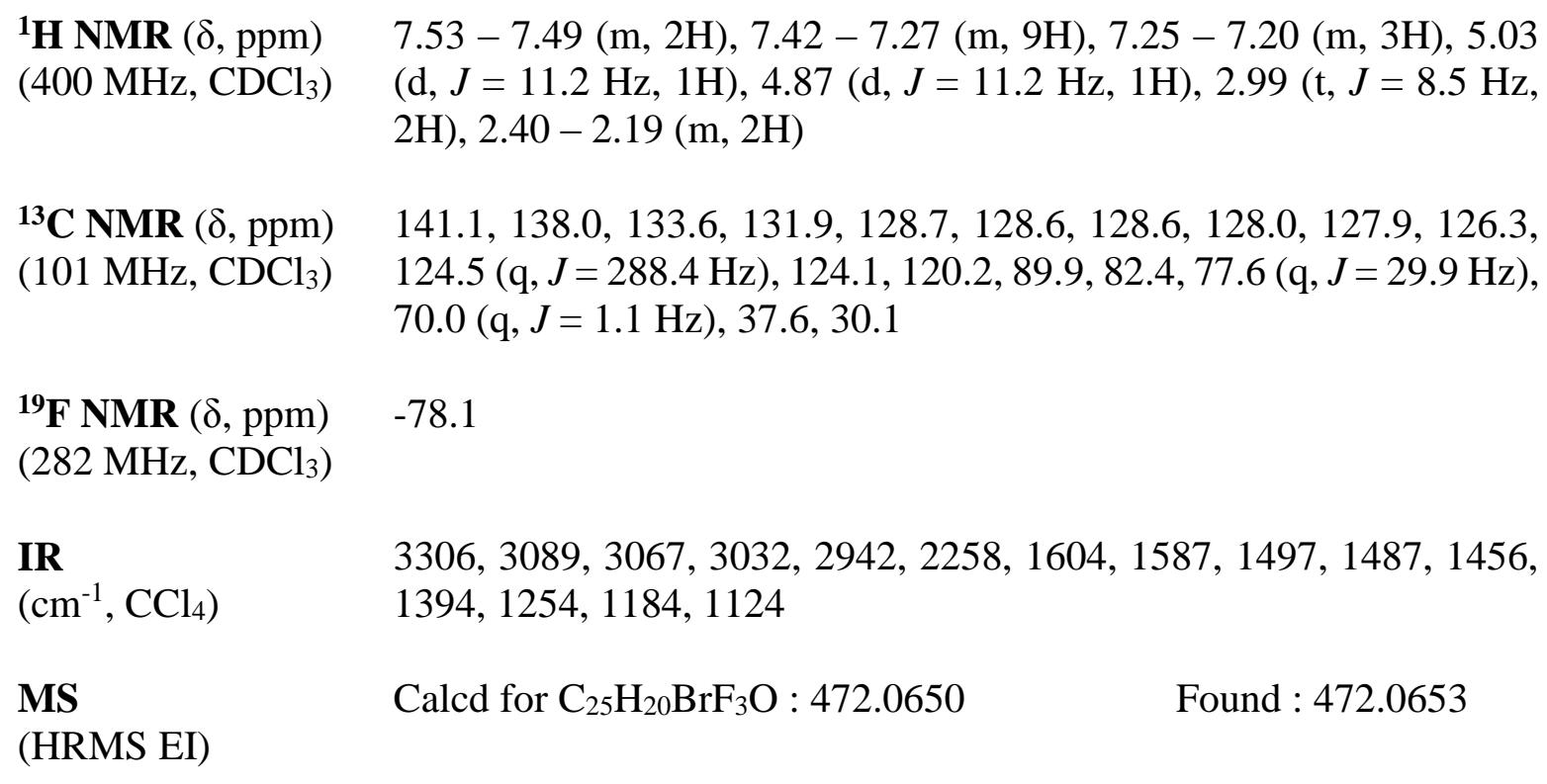

\section{(3-(benzyloxy)-3-(trifluoromethyl)non-4-ynyl)benzene (2u)}

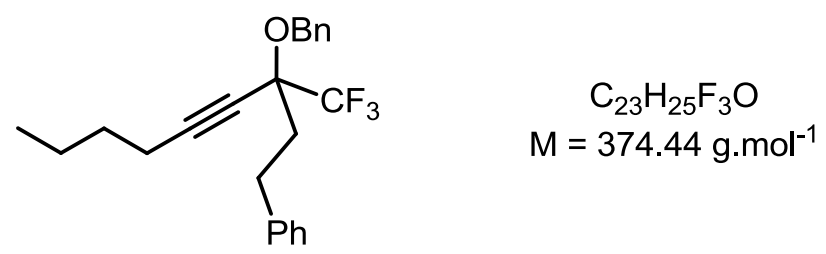

Following procedure B starting with 1-hexyne (2.5 mmol) and 1,1,1-trifluoro-4-phenyl-butan2-one.

Flash chromatography : PE / $\mathrm{Et}_{2} \mathrm{O} 100: 1$

Overall yield : $640 \mathrm{mg}(68 \%)$ of a yellow oil.

${ }^{1} \mathbf{H}$ NMR $(\delta, \mathrm{ppm}) \quad 7.31-7.24(\mathrm{~m}, 4 \mathrm{H}), 7.23-7.17(\mathrm{~m}, 3 \mathrm{H}), 7.14-7.06(\mathrm{~m}, 3 \mathrm{H}), 4.86$ $\left(400 \mathrm{MHz}, \mathrm{CDCl}_{3}\right) \quad(\mathrm{d}, J=11.2 \mathrm{~Hz}, 1 \mathrm{H}), 4.67(\mathrm{dd}, J=11.3,1.4 \mathrm{~Hz}, 1 \mathrm{H}), 2.87-2.77(\mathrm{~m}$, $2 \mathrm{H}), 2.25(\mathrm{t}, J=7.0 \mathrm{~Hz}, 2 \mathrm{H}), 2.17-1.95(\mathrm{~m}, 2 \mathrm{H}), 1.54-1.44(\mathrm{~m}, 2 \mathrm{H})$, $1.43-1.32(\mathrm{~m}, 2 \mathrm{H}), 0.86(\mathrm{t}, J=7.3 \mathrm{~Hz}, 3 \mathrm{H})$

${ }^{13}$ C NMR $(\delta, \mathrm{ppm}) \quad 141.5,138.4,128.6,128.6,128.5,127.9,127.8,126.1,124.7(\mathrm{q}, J=$ $\left.\left(101 \mathrm{MHz}, \mathrm{CDCl}_{3}\right) \quad 288.0 \mathrm{~Hz}\right), 92.4,77.2(\mathrm{q}, J=29.5 \mathrm{~Hz}), 72.6,69.6,37.8,30.5,30.1$, $22.0,18.5,13.6$

${ }^{19}$ F NMR $(\delta$, ppm $) \quad-78.7$

$\left(282 \mathrm{MHz}, \mathrm{CDCl}_{3}\right)$

IR

$\left(\mathrm{cm}^{-1}, \mathrm{CCl}_{4}\right)$
3089, 3067, 3031, 2961, 2937, 2875, 2251, 1604, 1497, 1456, 1381, $1261,1182,1148,1101,1052$ 
(HRMS EI)

(3-(benzyloxy)-3-(perfluoroethyl)non-4-ynyl)benzene (2v)

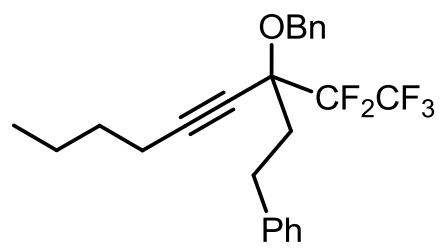

$$
\begin{gathered}
\mathrm{C}_{24} \mathrm{H}_{25} \mathrm{~F}_{5} \mathrm{O} \\
\mathrm{M}=424.45 \mathrm{~g} \cdot \mathrm{mol}^{-1}
\end{gathered}
$$

Following procedure B starting with 1-hexyne (1.3 eq), $n$-BuLi (1.2 eq) and 1,1,1,2,2pentafluoro-5-phenylpentan-3-one (1 eq) (prepared according to the literature procedure using ethyl pentafluoropropionate instead of ethyl trifluoroacetate) in THF (0.3 M).

Flash chromatography : PE / $\mathrm{Et}_{2} \mathrm{O} 100: 1$

Overall yield : $54 \%$

${ }^{1} \mathbf{H}$ NMR $(\delta, \mathrm{ppm}) \quad 7.39-7.29(\mathrm{~m}, 7 \mathrm{H}), 7.24-7.20(\mathrm{~m}, 3 \mathrm{H}), 4.92(\mathrm{~d}, J=11.1 \mathrm{~Hz}, 1 \mathrm{H})$, $\left(400 \mathrm{MHz}, \mathrm{CDCl}_{3}\right) \quad 4.78(\mathrm{~d}, J=11.1 \mathrm{~Hz}), 3.00-2.86(\mathrm{~m}, 2 \mathrm{H}), 2.39(\mathrm{t}, J=6.9 \mathrm{~Hz}, 2 \mathrm{H})$, $2.31-2.23(\mathrm{~m}, 1 \mathrm{H}), 2.19-2.11(\mathrm{~m}, 1 \mathrm{H}), 1.64-1.57(\mathrm{~m}, 2 \mathrm{H}), 1.54-$ $1.45(\mathrm{~m}, 2 \mathrm{H}), 0.97(\mathrm{t}, J=7.3 \mathrm{~Hz}, 3 \mathrm{H})$

${ }^{13} \mathbf{C ~ N M R}(\delta, \mathrm{ppm}) \quad 141.4,138.1,128.6,128.6,128.5,128.0,127.9,126.2,119.3(\mathrm{qt}, J=$ $\left.\left(101 \mathrm{MHz}, \mathrm{CDCl}_{3}\right) \quad 288.5,35.9 \mathrm{~Hz}\right), 113.9(\mathrm{tq}, J=264.1,35.1 \mathrm{~Hz}), 93.5,78.1(\mathrm{t}, J=23.3$ $\mathrm{Hz}), 72.2,69.6,37.9,30.5$ (x2), 22.0, 18.6, 13.7

${ }^{19}$ F NMR $(\delta, \mathrm{ppm}) \quad-79.4(\mathrm{~s}),-118.7(\mathrm{~d}, J=276.5 \mathrm{~Hz}),-120.2(\mathrm{~d}, J=275.7 \mathrm{~Hz})$ $\left(282 \mathrm{MHz}, \mathrm{CDCl}_{3}\right)$

IR

$\left(\mathrm{cm}^{-1}, \mathrm{CCl}_{4}\right)$

MS

(HRMS EI)
3089, 3067, 3031, 2961, 2937, 2875, 2259, 1604, 1497, 1455, 1431, $1381,1342,1276,1217,1183,1143,1098,1074,1028$

Calcd for $\mathrm{C}_{24} \mathrm{H}_{25} \mathrm{~F}_{5} \mathrm{O}: 424.1826$

Found : 424.1840

(2-(benzyloxy)-1,1,1-trifluoronon-3-yn-2-yl)benzene (2w)<smiles>CCCCCC#CC(OCc1ccccc1)(c1ccccc1)C(F)(F)F</smiles>

$$
\begin{gathered}
\mathrm{C}_{22} \mathrm{H}_{23} \mathrm{~F}_{3} \mathrm{O} \\
M=360.41 \mathrm{~g} \cdot \mathrm{mol}^{-1}
\end{gathered}
$$


Following procedure B starting with $n$-heptyne and 2,2,2-trifluoroacetophenone

Flash chromatography : pure PE to PE/EtOAc $98: 2$.

Overall yield : $558 \mathrm{mg}(77 \%)$ of a colorless oil.

${ }^{1} \mathbf{H}$ NMR $(\delta, \mathrm{ppm}) \quad 7.83-7.80(\mathrm{~m}, 2 \mathrm{H}), 7.47-7.31(\mathrm{~m}, 8 \mathrm{H}), 4.84(\mathrm{~d}, J=11.3 \mathrm{~Hz}, 1 \mathrm{H})$,

$\left(400 \mathrm{MHz}, \mathrm{CDCl}_{3}\right) \quad 4.44(\mathrm{~d}, J=11.1 \mathrm{~Hz}, 1 \mathrm{H}), 2.42(\mathrm{t}, J=7.1 \mathrm{~Hz}, 2 \mathrm{H}), 1.69-1.62(\mathrm{~m}, 2 \mathrm{H})$, $1.51-1.45(\mathrm{~m}, 2 \mathrm{H}), 1.44-1.34(\mathrm{~m}, 2 \mathrm{H}), 0.95(\mathrm{t}, J=7.2 \mathrm{~Hz}, 3 \mathrm{H})$

${ }^{13} \mathbf{C ~ N M R}(\delta, \mathrm{ppm}) \quad 137.8,134.5,129.6,128.5,128.5,128.4,127.8,127.6,123.3(\mathrm{q}, J=$ $\left.\left(101 \mathrm{MHz}, \mathrm{CDCl}_{3}\right) \quad 285.0 \mathrm{~Hz}\right), 92.6,79.6(\mathrm{q}, J=31.4 \mathrm{~Hz}), 73.2,67.5,31.2,28.0,22.2$, $18.9,14.1$

${ }^{19} \mathbf{F}$ NMR $(\delta, \mathrm{ppm}) \quad-79.6$

$\left(282 \mathrm{MHz}, \mathrm{CDCl}_{3}\right)$

IR $3069,3034,2960,2934,2863,2245,1451,1276,1189,1070,1030$

$\left(\mathrm{cm}^{-1}, \mathrm{CCl}_{4}\right)$

MS Calcd for $\mathrm{M}: \mathrm{C}_{22} \mathrm{H}_{23} \mathrm{~F}_{3} \mathrm{O}: 360.1701 \quad$ Found : 360.1690

(HRMS EI)

(3-(benzyloxy)-4,4,4-trifluorobut-1-yne-1,3-diyl)dibenzene $(2 x)$

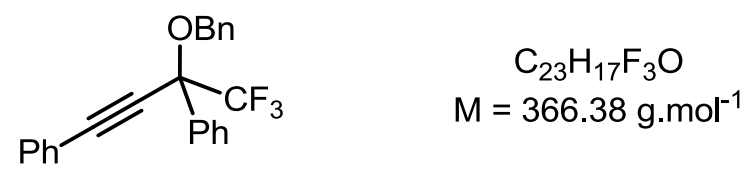

Following a similar protocol of procedure B starting with commercial phenylacetylene and 2,2,2-trifluoroacetophenone $(2.00 \mathrm{mmol})$

Flash chromatography : pure PE to PE/EtOAc 95 : 5.

Overall yield : $580 \mathrm{mg}$ (79\%) of a white solid.

${ }^{1} \mathbf{H}$ NMR $(\delta, \mathrm{ppm}) \quad 7.92-7.82(\mathrm{~m}, 2 \mathrm{H}), 7.62-7.59(\mathrm{~m}, 2 \mathrm{H}), 7.52-7.33(\mathrm{~m}, 11 \mathrm{H}), 4.95$ $\left(300 \mathrm{MHz}, \mathrm{CDCl}_{3}\right) \quad(\mathrm{d}, J=11.3 \mathrm{~Hz}, 1 \mathrm{H}), 4.57(\mathrm{~d}, J=11.3 \mathrm{~Hz}, 1 \mathrm{H})$

${ }^{13} \mathbf{C ~ N M R}(\delta, \mathrm{ppm}) \quad 137.6,134.0,132.3,129.9,129.7,128.6,128.5,128.5,128.5,127.9$, $\left(101 \mathrm{MHz}, \mathrm{CDCl}_{3}\right) \quad 127.7,123.2(\mathrm{q}, J=285.2 \mathrm{~Hz}), 121.3,91.1,81.8,80.1(\mathrm{q}, J=31.4 \mathrm{~Hz})$, 67.8

${ }^{19} \mathbf{F}$ NMR $(\delta, \mathrm{ppm}) \quad-79.2$

$\left(282 \mathrm{MHz}, \mathrm{CDCl}_{3}\right)$ 
IR

$\left(\mathrm{cm}^{-1}, \mathrm{CCl}_{4}\right)$

MS

(HRMS EI)

MP
$3068,3034,2930,2873,2234,1600,1451,1491,1280,1204,1177$, 1098,1061

Calcd for $\mathrm{M}: \mathrm{C}_{23} \mathrm{H}_{17} \mathrm{~F}_{3} \mathrm{O}: 366.1231$

$46.5-47.0^{\circ} \mathrm{C}$

4-(benzyloxy)-5,5,5-trifluoro-4-phenylpent-2-yn-1-ol (2y)<smiles>CCOC(C#CCO)(c1ccccc1)C(F)(F)c1ccccc1</smiles>

Following procedure D starting with $\mathbf{2 h}(2 \mathrm{mmol})$ and paraformaldehyde $(5 \mathrm{eq})$.

Flash chromatography : PE/EtOAc $75: 25$.

Overall yield : $490 \mathrm{mg}(75 \%)$ of a colorless oil.

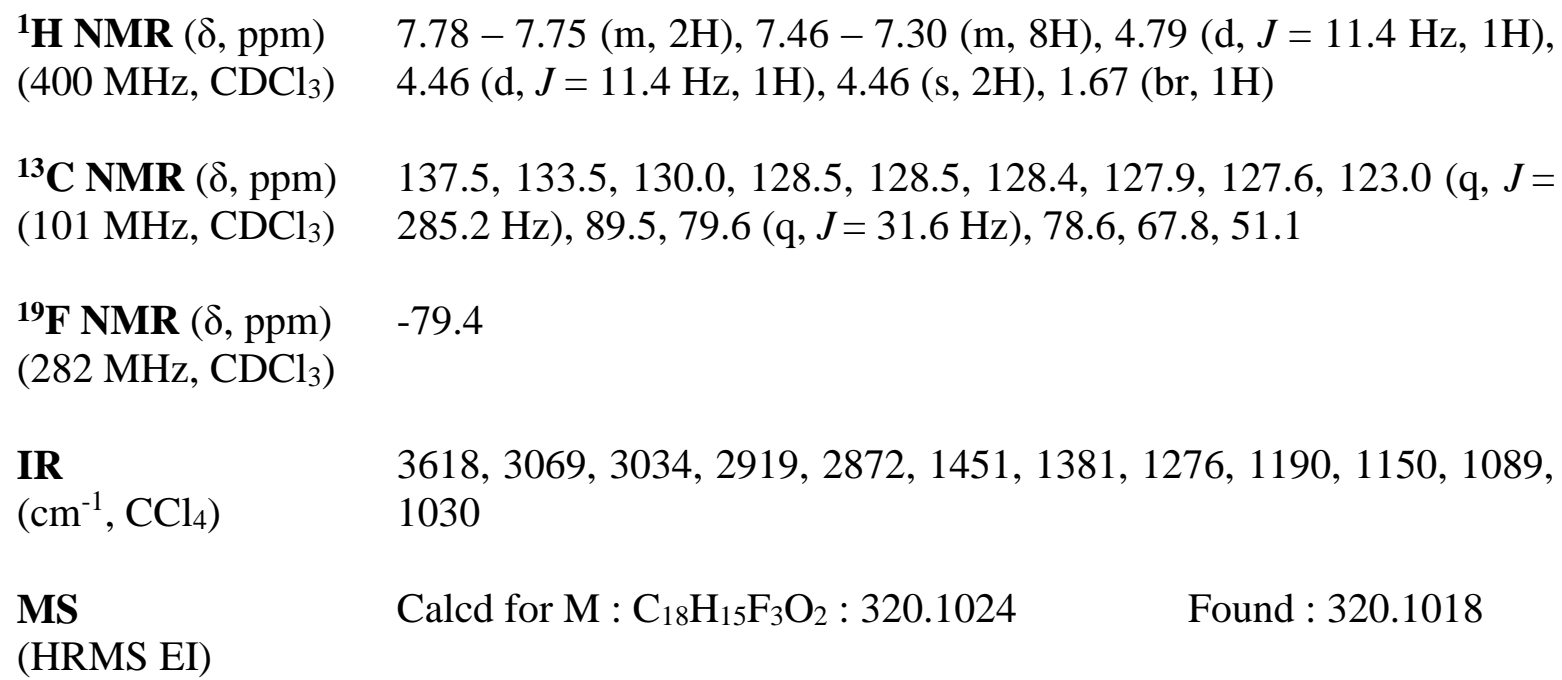

6-(benzyloxy)-7,7,7-trifluoro-2-methyl-6-phenylhept-4-yn-3-ol (2z)

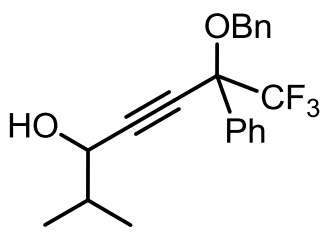

$$
\begin{gathered}
\mathrm{C}_{21} \mathrm{H}_{21} \mathrm{~F}_{3} \mathrm{O}_{2} \\
M=362.39 \mathrm{~g} \cdot \mathrm{mol}^{-1}
\end{gathered}
$$

Following procedure D starting with $\mathbf{2 h}(1 \mathrm{mmol})$ and isobutyraldehyde $(1.5 \mathrm{eq})$.

Flash chromatography : PE/EtOAc $85: 15$. 
Overall yield : $224 \mathrm{mg}(62 \%)$ of a colorless oil. The product was obtained as a mixture of two diastereomers.

${ }^{1} \mathbf{H}$ NMR $(\delta, \mathrm{ppm}) \quad 7.78-7.76(\mathrm{~m}, 2 \mathrm{H}), 7.46-7.44(\mathrm{~m}, 3 \mathrm{H}), 7.40-7.32(\mathrm{~m}, 5 \mathrm{H}), 4.79$

$\left(400 \mathrm{MHz}, \mathrm{CDCl}_{3}\right) \quad(\mathrm{d}, J=11.0 \mathrm{~Hz}, 1 \mathrm{H}), 4.47^{*}(\mathrm{~d}, J=11.4 \mathrm{~Hz} 1 \mathrm{H}), 4.38-4.36(\mathrm{~m}, 1 \mathrm{H})$, $2.05-1.96(\mathrm{~m}, 1 \mathrm{H}), 1.83$ (br, $1 \mathrm{H}), 1.05(\mathrm{~m}, 6 \mathrm{H})$

${ }^{13}$ C NMR $(\delta, \mathrm{ppm}) \quad 137.4^{*}, 133.5^{*}, 129.8,128.4,128.4,128.2,127.8,127.4,123.0(\mathrm{q}, J$ $\left.\left(101 \mathrm{MHz}, \mathrm{CDCl}_{3}\right) \quad=285.0 \mathrm{~Hz}\right), 91.1^{*}, 79.5(\mathrm{q}, J=31.4 \mathrm{~Hz}), 78.3,67.8,67.6,34.5,18.1$, 17.3

${ }^{19} \mathbf{F}$ NMR $(\delta, \mathrm{ppm}) \quad-79.5$

$\left(282 \mathrm{MHz}, \mathrm{CDCl}_{3}\right)$

IR $3619,3069,3034,2963,2928,2874,2856,1498,1467,1451,1275$, $\left(\mathrm{cm}^{-1}, \mathrm{CCl}_{4}\right) \quad 1190,1151,1071,1031,959$

MS Calcd for $\mathrm{M}: \mathrm{C}_{21} \mathrm{H}_{21} \mathrm{~F}_{3} \mathrm{O}_{2}: 362.1494 \quad$ Found : 362.1481 (HRMS EI)

* Observed as doublets due to the slight chemical shift difference between the two diastereomers.

\section{Catalysis step}

\section{General procedure E :}<smiles>[R]C#CC([R])(C#[R])OCc1nccs1</smiles>

2a-w

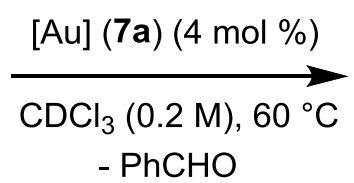

$-\mathrm{PhCHO}$<smiles>[R]C(=C=[W])C([R])(F)F</smiles>

4a-w

To a solution of propargylic benzylic ether $(1 \mathrm{eq})$ in dry $\mathrm{CDCl}_{3}\left(0.2 \mathrm{~mol} . \mathrm{L}^{-1}\right)$ was added PhosphoniteAuSbF $6(0.04 \mathrm{eq})$ and the reaction was heated up to $60^{\circ} \mathrm{C}$. Upon completion on the reaction (NMR), the solvent was evaporated and purification by flash chromatography gave the pure trifluoromethyl allene.

Crude NMR yield was provided by adding 1,2-dichloroethane as an internal standard. 


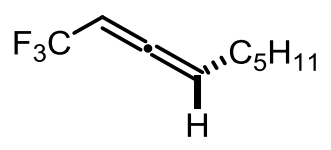

$$
\begin{gathered}
\mathrm{C}_{9} \mathrm{H}_{13} \mathrm{~F}_{3} \\
M=178.19 \mathrm{~g} \cdot \mathrm{mol}^{-1}
\end{gathered}
$$

Following general procedure E starting with $2 \mathbf{a}(0.20 \mathrm{mmol}$, reaction time : $1 \mathrm{~h})$.

Flash chromatography : pentane

Yield : Colorless oil (crude NMR yield : $85 \%$ ).

The product could not be isolated because of its low boiling point. Some pentane remains present on the spectra.

${ }^{1} \mathbf{H}$ NMR $(\delta, \mathrm{ppm}) \quad 5.71-5.63(\mathrm{~m}, 1 \mathrm{H}), 5.44-5.37(\mathrm{~m}, 1 \mathrm{H}), 2.14-2.08(\mathrm{~m}, 2 \mathrm{H}), 1.47-$ $\left(400 \mathrm{MHz}, \mathrm{CDCl}_{3}\right) \quad 1.43(\mathrm{~m}, 2 \mathrm{H}), 1.34-1.29(\mathrm{~m}, 4 \mathrm{H}), 0.91-0.88(\mathrm{~m}, 3 \mathrm{H})$

${ }^{13}$ C NMR $(\delta, \mathrm{ppm}) \quad 205.3(\mathrm{q}, J=5.8 \mathrm{~Hz}), 123.0(\mathrm{q}, J=270.2 \mathrm{~Hz}), 98.6,85.9(\mathrm{q}, J=38.9$ $\left.\left(101 \mathrm{MHz}, \mathrm{CDCl}_{3}\right) \quad \mathrm{Hz}\right), 31.2,28.3,27.7,22.5,14.1$

${ }^{19} \mathbf{F}$ NMR $(\delta, \mathrm{ppm}) \quad-61.6$

$\left(282 \mathrm{MHz}, \mathrm{CDCl}_{3}\right)$

MS Calcd for $\left[M-\mathrm{CF}_{3}\right] \mathrm{C}_{8} \mathrm{H}_{13}: 109.1017 \quad$ Found : 109.1020

(HRMS EI)

triisopropyl(7,7,7-trifluorohepta-4,5-dienyloxy)silane (4b)

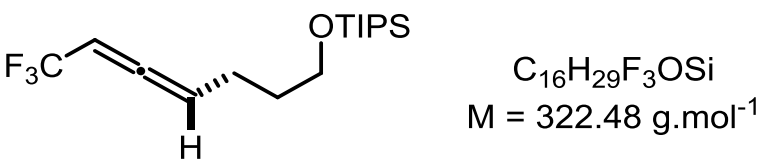

Following general procedure E starting with $\mathbf{2 b}(0.10 \mathrm{mmol}$, reaction time : $12 \mathrm{~h})$.

Flash chromatography : PE

Yield : $25.1 \mathrm{mg}(78 \%)$ of a yellow oil (crude NMR yield $89 \%$ ).

${ }^{1} \mathbf{H}$ NMR $(\delta, \mathrm{ppm}) \quad 5.77-5.68(\mathrm{~m}, 1 \mathrm{H}), 5.45-5.37(\mathrm{~m}, 1 \mathrm{H}), 3.73(\mathrm{t}, J=6.1 \mathrm{~Hz}, 2 \mathrm{H}), 2.23$ $\left(400 \mathrm{MHz}, \mathrm{CDCl}_{3}\right) \quad(\mathrm{dtd}, J=8.8,8.0,3.2 \mathrm{~Hz}, 2 \mathrm{H}), 1.69(\mathrm{tt}, J=7.3,6.1 \mathrm{~Hz}, 2 \mathrm{H}), 1.09-$ $1.00(\mathrm{~m}, 21 \mathrm{H})$

${ }^{13}$ C NMR $(\delta, \mathrm{ppm}) \quad 205.2(\mathrm{q}, J=5.8 \mathrm{~Hz}), 123.0(\mathrm{q}, J=270.1 \mathrm{~Hz}), 98.5,86.2(\mathrm{q}, J=39.0$ $\left.\left(101 \mathrm{MHz}, \mathrm{CDCl}_{3}\right) \quad \mathrm{Hz}\right), 62.3,31.8,24.2(\mathrm{~d}, J=1.5 \mathrm{~Hz}), 18.1,12.1$ 


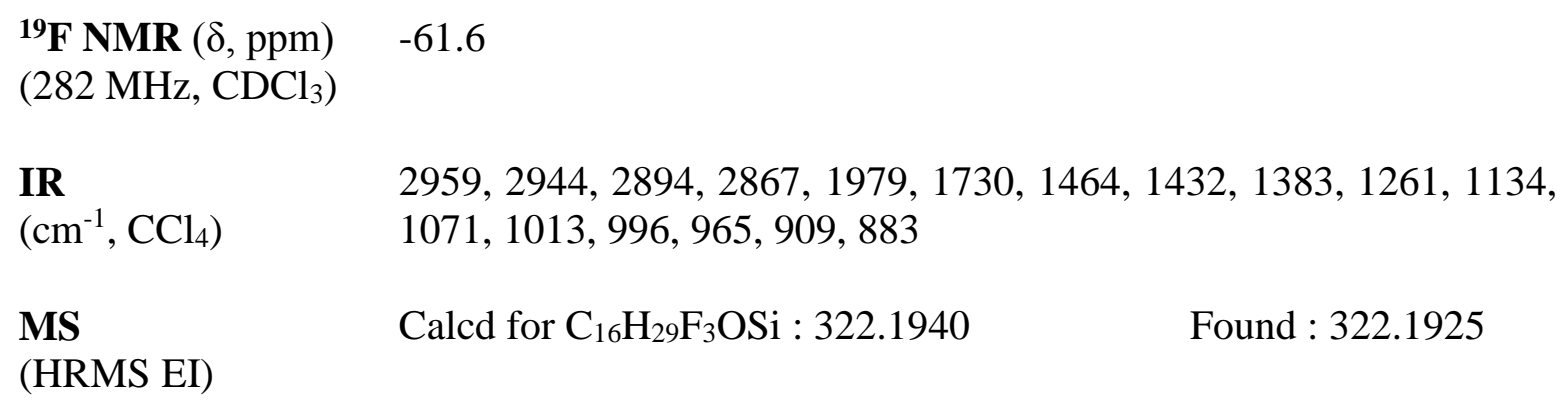

Following general procedure E starting with $2 c(0.20 \mathrm{mmol}$, reaction time : $12 \mathrm{~h})$.

Flash chromatography : PE / $\mathrm{Et}_{2} \mathrm{O} 50: 1$

Yield : $30.5 \mathrm{mg}(60 \%)$ of a colorless oil (crude NMR yield $65 \%$ ).

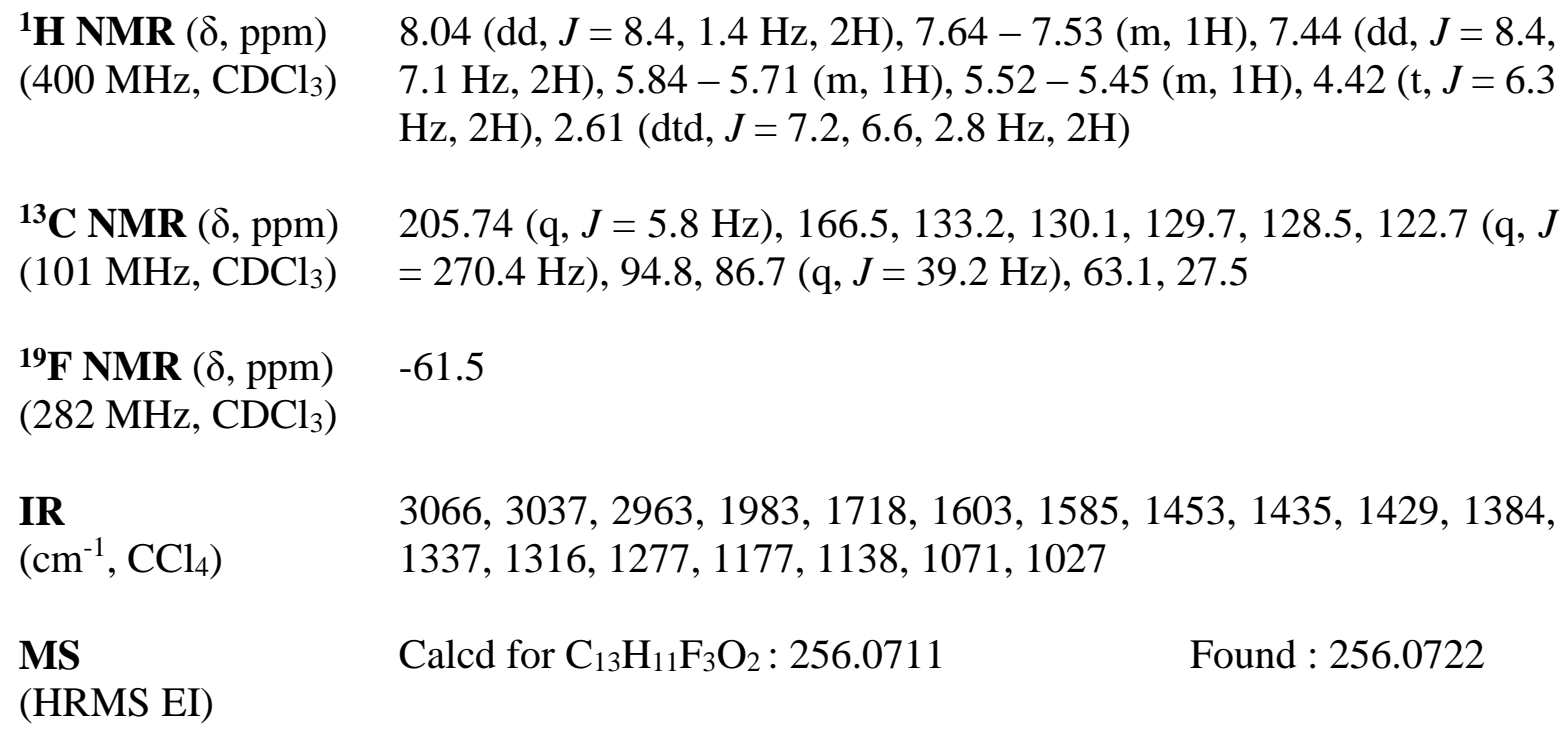

Following general procedure E starting with $\mathbf{2 d}(0.20 \mathrm{mmol}$, reaction time $: 6 \mathrm{~h})$. 
Flash chromatography : $\mathrm{PE} / \mathrm{Et}_{2} \mathrm{O} 50: 1$

Yield : $20.8 \mathrm{mg}$ (43\%) of a colorless oil (crude NMR yield $50 \%$ ).

${ }^{1} \mathbf{H}$ NMR $(\delta, \mathrm{ppm}) \quad 7.40-7.27(\mathrm{~m}, 5 \mathrm{H}), 5.81-5.72(\mathrm{~m}, 1 \mathrm{H}), 5.49-5.41(\mathrm{~m}, 1 \mathrm{H}), 4.53$

$\left(400 \mathrm{MHz}, \mathrm{CDCl}_{3}\right) \quad(\mathrm{s}, 2 \mathrm{H}), 3.59(\mathrm{t}, J=6.4 \mathrm{~Hz}, 2 \mathrm{H}), 2.45(\mathrm{dtd}, J=7.4,6.4,3.0 \mathrm{~Hz}, 2 \mathrm{H})$

${ }^{13} \mathbf{C ~ N M R}(\delta, \mathrm{ppm}) \quad 205.5(\mathrm{q}, J=5.8 \mathrm{~Hz}), 138.2,128.6,127.8,127.8,122.9(\mathrm{q}, J=270.1$

$\left.\left(101 \mathrm{MHz}, \mathrm{CDCl}_{3}\right) \quad \mathrm{Hz}\right), 95.6,86.2(\mathrm{q}, J=38.9 \mathrm{~Hz}), 73.2,68.8,28.4$

${ }^{19}$ F NMR $(\delta, \mathrm{ppm}) \quad-61.5$

$\left(282 \mathrm{MHz}, \mathrm{CDCl}_{3}\right)$

IR

$3067,3033,2958,2864,2258,1981,1495,1455,1429,1364,1298$,

$\left(\mathrm{cm}^{-1}, \mathrm{CCl}_{4}\right)$ $1261,1211,1136$

$\begin{array}{lll}\text { MS Calcd for } \mathrm{C}_{13} \mathrm{H}_{13} \mathrm{~F}_{3} \mathrm{O}: 242.0918 & \text { Found : } 242.0903\end{array}$

(HRMS EI)

2-(7,7,7-trifluorohepta-4,5-dienyl)isoindoline-1,3-dione (4e)

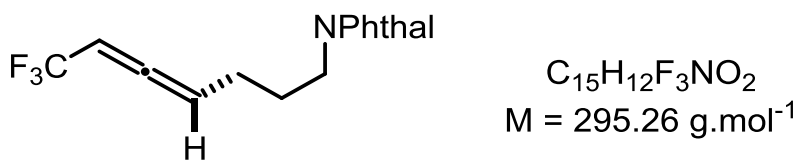

Following general procedure E starting with $2 \mathrm{e}(0.20 \mathrm{mmol}$, reaction time : $12 \mathrm{~h})$.

Flash chromatography : $\mathrm{PE} / \mathrm{Et}_{2} \mathrm{O} 10: 1$

Yield : $51.9 \mathrm{mg}(88 \%)$ of a white solid (crude NMR yield $92 \%)$.

${ }^{1} \mathbf{H}$ NMR $(\delta, \mathrm{ppm}) \quad 7.84(\mathrm{dd}, J=5.5,3.1 \mathrm{~Hz}, 2 \mathrm{H}), 7.72(\mathrm{dd}, J=5.5,3.1 \mathrm{~Hz}, 2 \mathrm{H}), 5.77-$ $\left(400 \mathrm{MHz}, \mathrm{CDCl}_{3}\right) \quad 5.68(\mathrm{~m}, 1 \mathrm{H}), 5.49-5.41(\mathrm{~m}, 1 \mathrm{H}), 3.73(\mathrm{td}, J=7.0,1.2 \mathrm{~Hz}, 2 \mathrm{H}), 2.26$ $-2.11(\mathrm{dtd}, J=8.8,7.2,3.2 \mathrm{~Hz}, 2 \mathrm{H}), 1.92-1.75(\mathrm{tt}, J=8.4,7.2 \mathrm{~Hz}$, $2 \mathrm{H})$

${ }^{13}$ C NMR $(\delta, \mathrm{ppm}) \quad 205.2(\mathrm{q}, J=5.8 \mathrm{~Hz}), 168.5,134.1,132.2,123.4,122.9(\mathrm{q}, J=270.3$ $\left.\left(101 \mathrm{MHz}, \mathrm{CDCl}_{3}\right) \quad \mathrm{Hz}\right), 97.5,86.7(\mathrm{q}, J=39.0 \mathrm{~Hz}), 37.3,27.5,25.1$

${ }^{19}$ F NMR $(\delta, \mathrm{ppm}) \quad-61.6$

$\left(282 \mathrm{MHz}, \mathrm{CDCl}_{3}\right)$

IR

$\left(\mathrm{cm}^{-1}, \mathrm{CCl}_{4}\right)$

MS

(HRMS EI)
3155, 2984, 2951, 2254, 1981, 1817, 1795, 1774, 1718, 1642, 1469, 1437, 1396, 1297, 1263, 1170, 1133, 1098, 1035, 992, 908

Calcd for $\mathrm{C}_{15} \mathrm{H}_{12} \mathrm{~F}_{3} \mathrm{NO}_{2}: 295.0820$

Found : 295.0825 
1-methoxy-4-(4,4,4-trifluorobuta-1,2-dienyl)benzene (4f)<smiles>COc1ccc(C=C=CC(F)(F)F)cc1</smiles>

Following general procedure E starting with $\mathbf{2 f}(0.08 \mathrm{mmol}$, reaction time : $7 \mathrm{~h})$.

Flash chromatography : pentane

Yield : $11.1 \mathrm{mg}(65 \%)$ of a yellow oil. (crude NMR yield $75 \%$ ).

${ }^{1} \mathbf{H}$ NMR $(\delta, \mathrm{ppm}) \quad 7.24(\mathrm{~d}, J=8.7 \mathrm{~Hz}, 2 \mathrm{H}), 6.89(\mathrm{~d}, J=8.7 \mathrm{~Hz}, 2 \mathrm{H}), 6.66-6.61(\mathrm{~m}, 1 \mathrm{H})$, $\left(400 \mathrm{MHz}, \mathrm{CDCl}_{3}\right) \quad 5.88-5.82(\mathrm{~m}, 1 \mathrm{H}), 3.80(\mathrm{~s}, 3 \mathrm{H})$

${ }^{13}$ C NMR $(\delta, \mathrm{ppm}) \quad 206.8(\mathrm{q}, J=5.8 \mathrm{~Hz}), 160.1,128.9,123.0(\mathrm{~d}, J=1.5 \mathrm{~Hz}), 122.5(\mathrm{q}, J$ $\left.\left(101 \mathrm{MHz}, \mathrm{CDCl}_{3}\right) \quad=271.1 \mathrm{~Hz}\right), 114.6,101.0,89.6(\mathrm{q}, J=39.1 \mathrm{~Hz}), 55.5$

${ }^{19} \mathbf{F}$ NMR $(\delta$, ppm $) \quad-61.3$

$\left(282 \mathrm{MHz}, \mathrm{CDCl}_{3}\right)$

IR

$\left(\mathrm{cm}^{-1}, \mathrm{CCl}_{4}\right)$

MS

(HRMS EI)
2959, 2934, 1966, 1607, 1513, 1465, 1441, 1407, 1319, 1302, 1277, $1252,1174,1134,1033$

Calcd for $\mathrm{C}_{11} \mathrm{H}_{9} \mathrm{~F}_{3} \mathrm{O}: 214.0605$

Found : 214.0610

\section{(3-(trifluoromethyl)penta-3,4-dienyl)benzene (4g)}

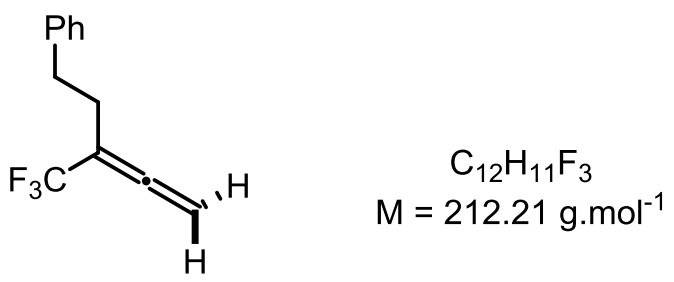

Following general procedure E starting with $2 \mathrm{~g}(0.20 \mathrm{mmol}$, reaction time : $1 \mathrm{~h})$.

Flash chromatography : PE

Yield : $36.9 \mathrm{mg}(87 \%)$ of a yellowish oil (crude NMR yield $91 \%$ ). 


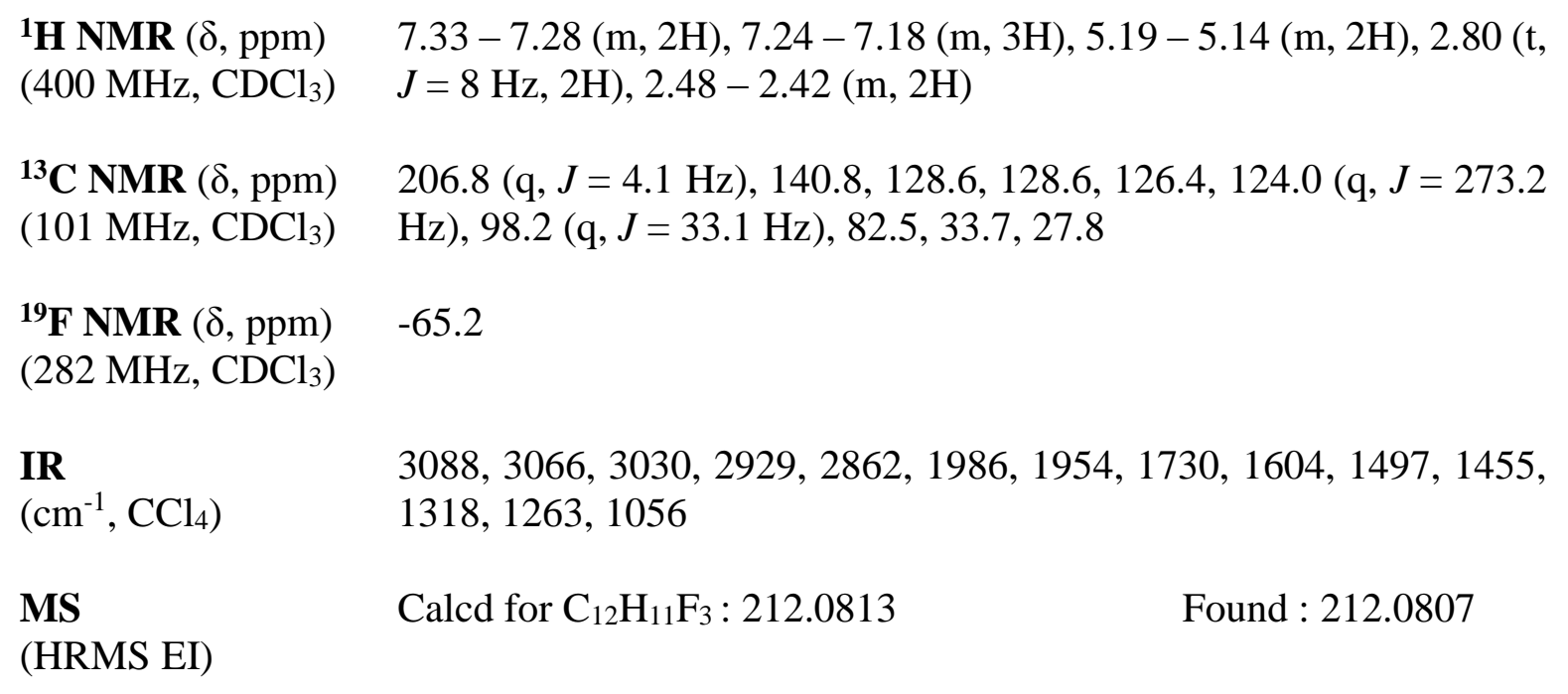

\section{(1,1,1-trifluorobuta-2,3-dien-2-yl)benzene (4h)}

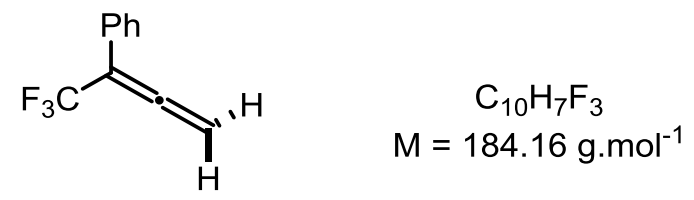

Following general procedure E starting with $\mathbf{2 h}(0.20 \mathrm{mmol}$, reaction time : $1 \mathrm{~h})$.

Flash chromatography : pentane

Yield : Colorless oil (crude NMR yield : $90 \%$ ).

The product could not be isolated because of its low boiling point. Some pentane remains present on the spectra.

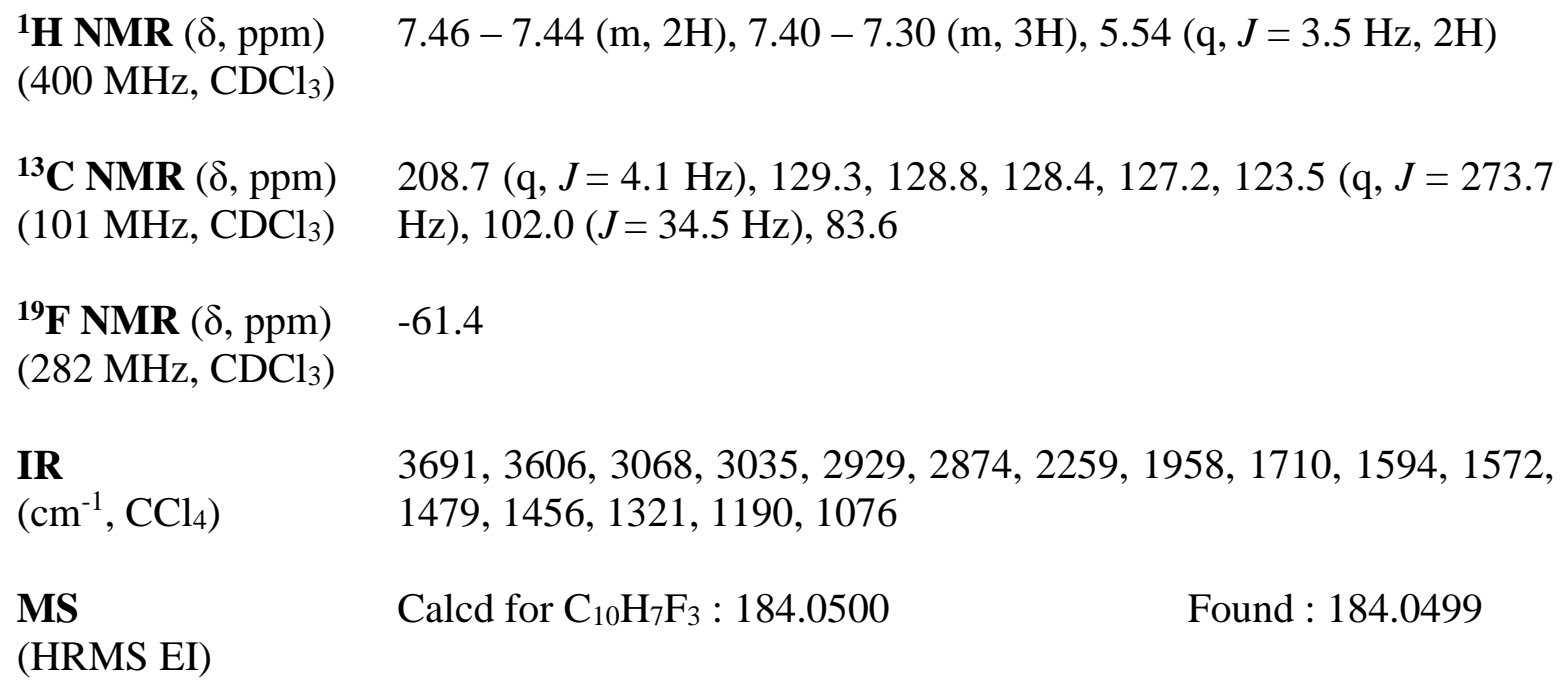




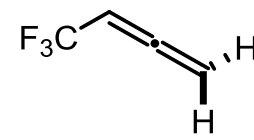

$$
\begin{gathered}
\mathrm{C}_{4} \mathrm{H}_{3} \mathrm{~F}_{3} \\
\mathrm{M}=108.06 \mathrm{~g} \cdot \mathrm{mol}^{-1}
\end{gathered}
$$

Following general procedure $\mathrm{E}$ starting with $2 \mathbf{i}$ ( $2 \mathbf{i}$ in solution in $\mathrm{Et}_{2} \mathrm{O}, 0.20 \mathrm{mmol}$, reaction time : $24 \mathrm{~h}$ ). The reaction was run in an NMR tube using 1,2-dichloroethane as an internal standard. The yield was estimated using this internal standard: crude NMR yield : $55 \%$

The product could not be isolated because of its low boiling point (boiling point calculated to be around $12^{\circ} \mathrm{C}$ ).

${ }^{1} \mathbf{H}$ NMR $(\delta, \mathrm{ppm}) \quad 5.51-5.43(\mathrm{~m}, 1 \mathrm{H}), 5.29-5.24(\mathrm{~m}, 2 \mathrm{H})$

$\left(400 \mathrm{MHz}, \mathrm{CDCl}_{3}\right)$

${ }^{13}$ C NMR $(\delta, \mathrm{ppm}) \quad 209.2(\mathrm{q}, J=5.8 \mathrm{~Hz}), 122.8(\mathrm{q}, J=269.9 \mathrm{~Hz}), 85.8(\mathrm{q}, J=39.3 \mathrm{~Hz})$, $\left(101 \mathrm{MHz}, \mathrm{CDCl}_{3}\right) \quad 82.0$

${ }^{19}$ F NMR $(\delta, \mathrm{ppm}) \quad-61.4$

$\left(282 \mathrm{MHz}, \mathrm{CDCl}_{3}\right)$

IR

2959, 2934, 1966, 1607, 1513, 1465, 1441, 1407, 1319, 1302, 1277,

$\left(\mathrm{cm}^{-1}, \mathrm{CCl}_{4}\right)$ $1252,1174,1134,1033$

MS

Calcd for $\mathrm{C}_{4} \mathrm{H}_{3} \mathrm{~F}_{3}: 108.0187$

Found : 108.0183

(HRMS EI)

(3R,10S,13R,17R)-10,13-dimethyl-12-oxo-17-((R)-5-(trifluoromethyl)hepta-5,6-dien-2yl)hexadecahydro-1H-cyclopenta[a]phenanthren-3-yl acetate (4j)

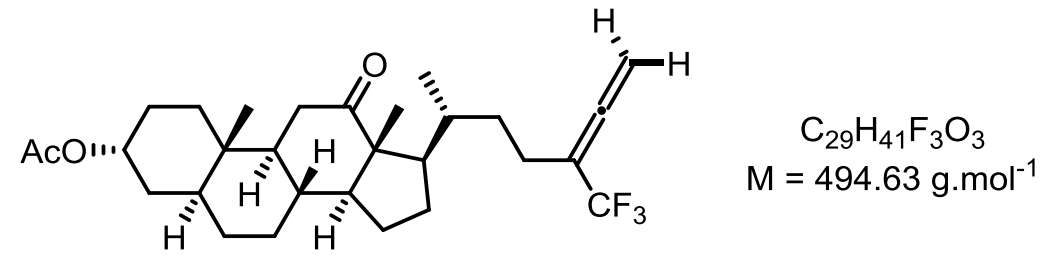

Following general procedure E starting with $\mathbf{2 j}$ (0.046 mmol, reaction time : $26 \mathrm{~h}$ ).

Flash chromatography : PE/EtOAc $90: 10$

Yield : $20.7 \mathrm{mg}(91 \%)$ of a white solid.

${ }^{1} \mathbf{H}$ NMR $(\delta, \mathrm{ppm}) \quad 5.20-5.15(\mathrm{~m}, 2 \mathrm{H}), 4.73-4.65(\mathrm{~m}, 1 \mathrm{H}), 2.48(\mathrm{t}, J=12.2 \mathrm{~Hz}, 1 \mathrm{H})$, $\left(400 \mathrm{MHz}, \mathrm{CDCl}_{3}\right) \quad 2.27-2.17(\mathrm{~m}, 1 \mathrm{H}), 2.06-2.01(\mathrm{~m}, 3 \mathrm{H}), 2.01(\mathrm{~s}, 3 \mathrm{H}), 1.92-1.02(\mathrm{~m}$, $21 \mathrm{H}), 1.02(\mathrm{~s}, 2 \mathrm{x} 3 \mathrm{H}), 0.87(\mathrm{~d}, J=5.9 \mathrm{~Hz}, 3 \mathrm{H})$ 


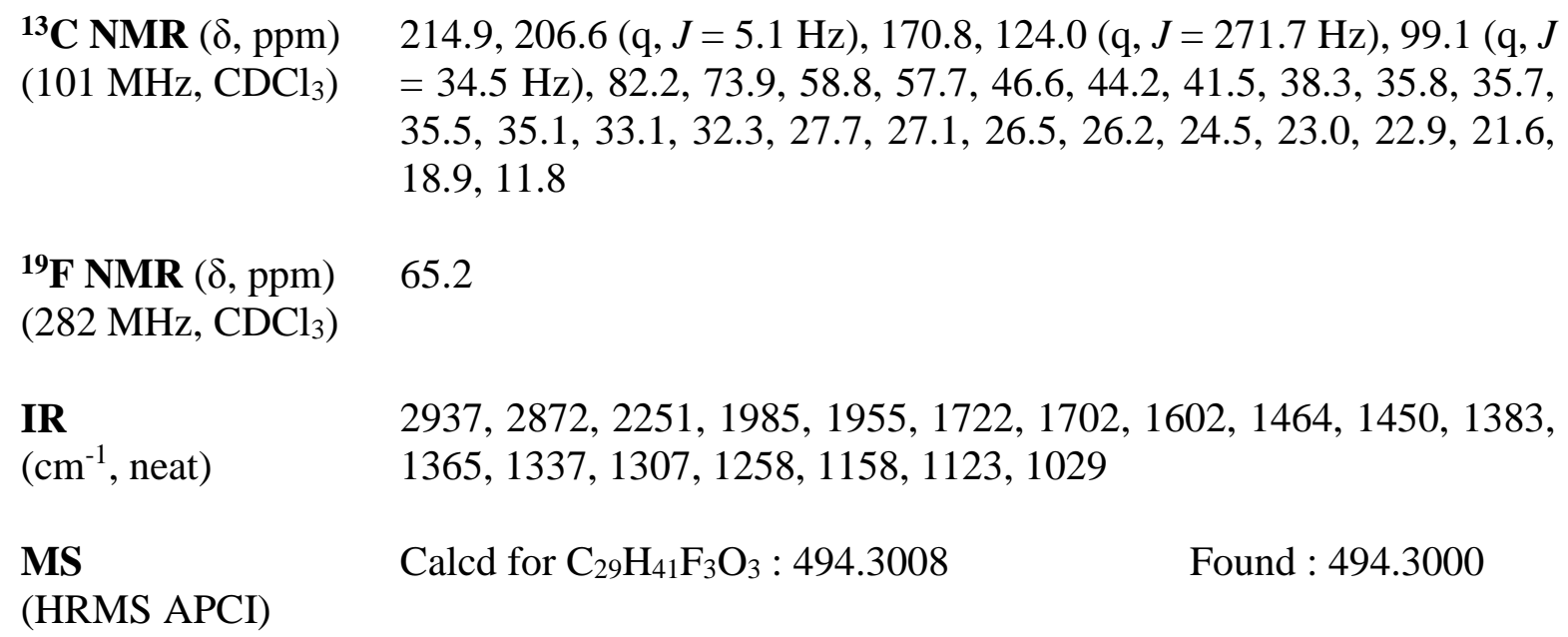

IR

$\left(\mathrm{cm}^{-1}\right.$, neat $)$

2937, 2872, 2251, 1985, 1955, 1722, 1702, 1602, 1464, 1450, 1383, $1365,1337,1307,1258,1158,1123,1029$

MS Calcd for $\mathrm{C}_{29} \mathrm{H}_{41} \mathrm{~F}_{3} \mathrm{O}_{3}: 494.3008 \quad$ Found : 494.3000

(HRMS APCI)

(7,7,7-trifluorohepta-4,5-dien-1-yl)benzene (4k)

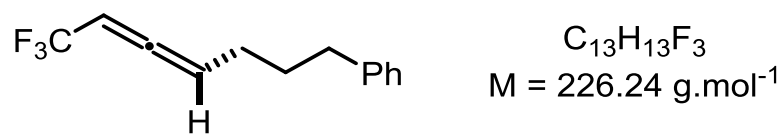

Following general procedure E starting with $2 \mathbf{k}(0.40 \mathrm{mmol}$, reaction time : $1 \mathrm{~h} 40)$ and 3 mol\% catalyst.

Flash chromatography : $\mathrm{PE} / \mathrm{Et}_{2} \mathrm{O} 96: 4$

Yield : $83.3 \mathrm{mg}(90 \%)$ of a yellow oil (crude NMR yield : $89 \%)$.

${ }^{1} \mathbf{H}$ NMR $(\delta, \mathrm{ppm}) \quad 7.32-7.28(\mathrm{~m}, 2 \mathrm{H}), 7.23-7.18(\mathrm{~m}, 3 \mathrm{H}), 5.74-5.66(\mathrm{~m}, 1 \mathrm{H}), 5.48-$

$\left(400 \mathrm{MHz}, \mathrm{CDCl}_{3}\right) \quad 5.41(\mathrm{~m}, 1 \mathrm{H}), 2.70-2.66(\mathrm{~m}, 2 \mathrm{H}), 2.19-2.13(\mathrm{~m}, 2 \mathrm{H}), 1.83-1.76$ $(\mathrm{m}, 2 \mathrm{H})$

${ }^{13} \mathbf{C ~ N M R}(\delta, \mathrm{ppm}) \quad 205.4(\mathrm{q}, J=5.9 \mathrm{~Hz}), 141.8,128.6,128.5,127.0,123.0(\mathrm{q}, J=270.0$

$\left.\left(101 \mathrm{MHz}, \mathrm{CDCl}_{3}\right) \quad \mathrm{Hz}\right), 98.3,86.2(\mathrm{q}, J=38.9 \mathrm{~Hz}), 30.5,30.2,27.1$

${ }^{19}$ F NMR $(\delta$, ppm $) \quad-61.5$

$\left(282 \mathrm{MHz}, \mathrm{CDCl}_{3}\right)$

IR

3029, 2938, 2861, 1979, 1497, 1454, 1297, 1260, 1134, 869

$\left(\mathrm{cm}^{-1}, \mathrm{CCl}_{4}\right)$

MS

Calcd for $\mathrm{C}_{13} \mathrm{H}_{13} \mathrm{~F}_{3}: 226.0969$

Found : 226.0973

(HRMS EI) 


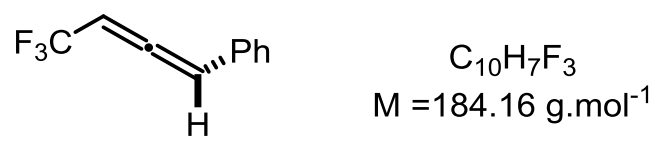

Following general procedure E starting with 21 ( $0.20 \mathrm{mmol}$, reaction time : $6 \mathrm{~h})$.

Flash chromatography : pentane

Yield : colorless oil (crude NMR yield $65 \%$ ).

The product could not be isolated because of its low boiling point. Some pentane remains present on the spectra.

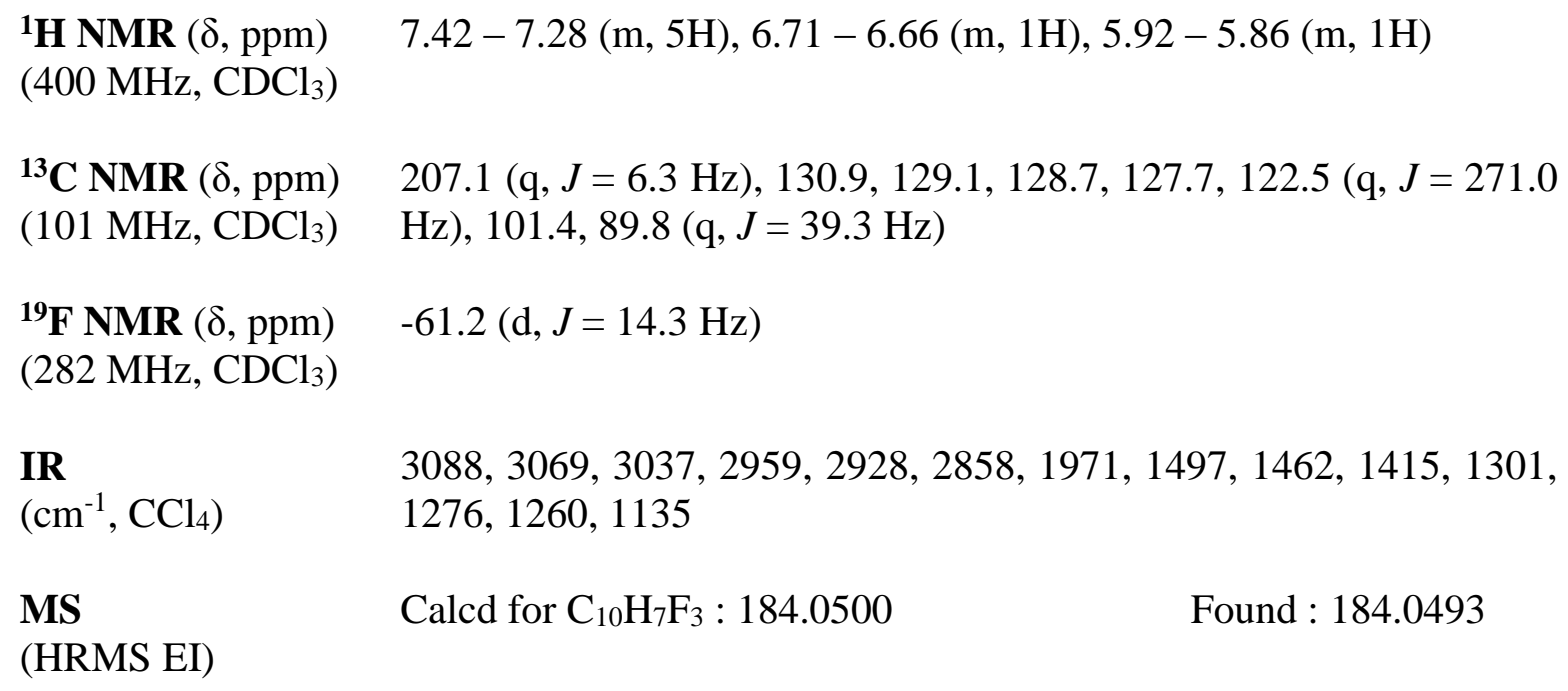

(7,7,8,8,8-pentafluoroocta-4,5-dienyl)benzene (4m)

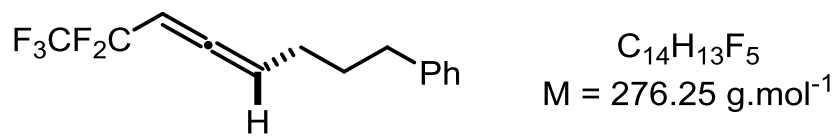

Following general procedure E starting with $\mathbf{2 m}(0.20 \mathrm{mmol}$, reaction time : $2 \mathrm{~h})$.

Flash chromatography : PE

Yield : $44.3 \mathrm{mg}$ (80\%) of a colorless oil (crude NMR yield $85 \%$ ).

${ }^{1} \mathbf{H}$ NMR $(\delta, \mathrm{ppm}) \quad 7.35-7.29(\mathrm{~m}, 2 \mathrm{H}), 7.25-7.13(\mathrm{~m}, 3 \mathrm{H}), 5.78-7.70(\mathrm{~m}, 1 \mathrm{H}), 5.45-$ $\left(400 \mathrm{MHz}, \mathrm{CDCl}_{3}\right) \quad 5.35(\mathrm{~m}, 1 \mathrm{H}), 2.69(\mathrm{t}, J=7.7 \mathrm{~Hz}, 2 \mathrm{H}), 2.18$ (tdd, $J=6.8,6.4,3.2 \mathrm{~Hz}$, $2 \mathrm{H}), 1.89-1.77(\mathrm{tt}, J=7.6,6.4 \mathrm{~Hz}, 2 \mathrm{H})$ 


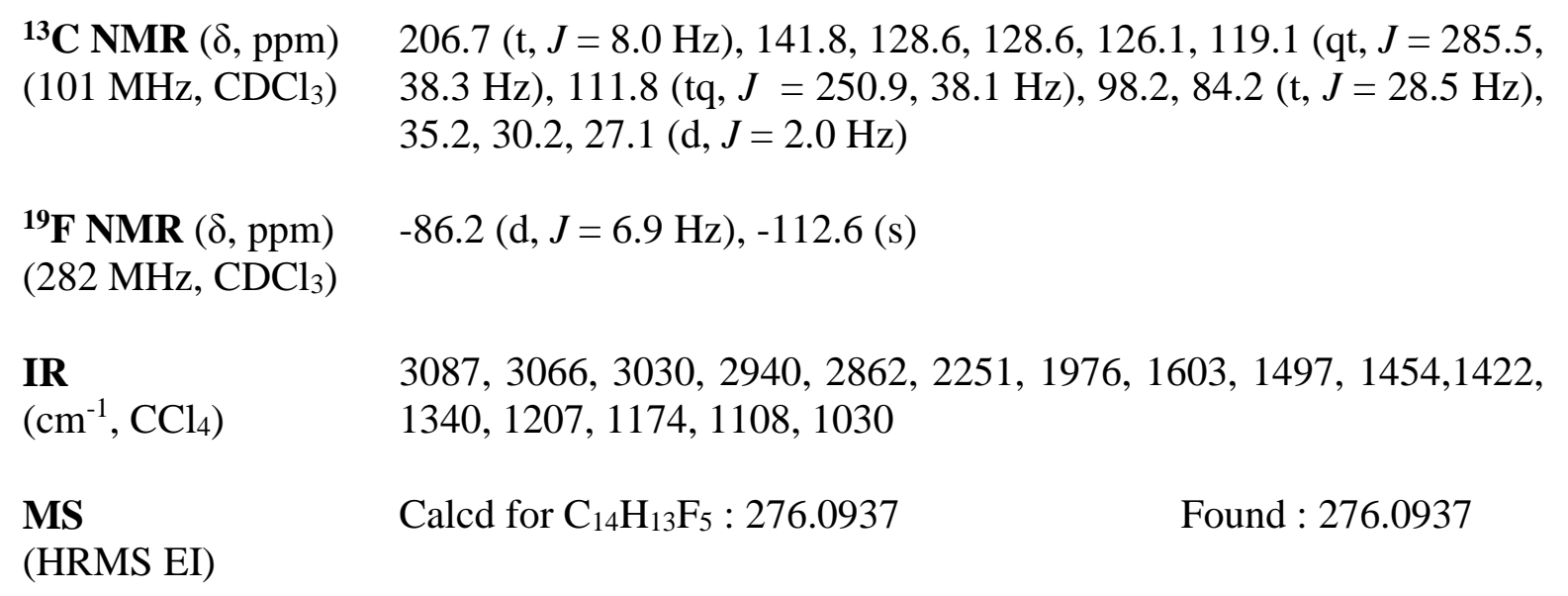

\section{(4,4,5,5,5-pentafluoropenta-1,2-dienyl)benzene (4n)}

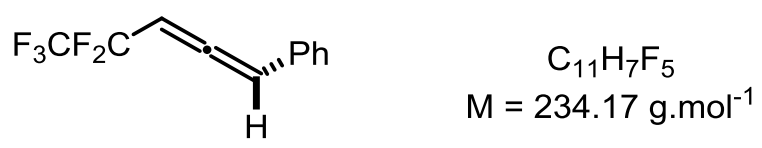

Following general procedure E starting with 2 n $(0.18 \mathrm{mmol}$, reaction time : $24 \mathrm{~h})$.

Flash chromatography : pentane.

Yield : yellow oil (crude NMR yield $85 \%$ ).

The product could not be isolated because of its low boiling point. Some pentane remains present on the spectra.

${ }^{1} \mathbf{H}$ NMR $(\delta, \mathrm{ppm}) \quad 7.40-7.35(\mathrm{~m}, 2 \mathrm{H}), 7.34-7.29(\mathrm{~m}, 3 \mathrm{H}), 6.72-6.67(\mathrm{~m}, 1 \mathrm{H}), 6.87-$ $\left(400 \mathrm{MHz}, \mathrm{CDCl}_{3}\right) \quad 6.80(\mathrm{~m}, 1 \mathrm{H})$

${ }^{13}$ C NMR $(\delta, \mathrm{ppm}) \quad 208.5(\mathrm{t}, J=8.0 \mathrm{~Hz}), 130.7(\mathrm{t}, J=1.9 \mathrm{~Hz}), 129.1,128.8,127.7,119.1$ $\left(101 \mathrm{MHz}, \mathrm{CDCl}_{3}\right) \quad(\mathrm{qt}, J=284.1,37.8 \mathrm{~Hz}), 111.4(\mathrm{tq}, J=232.2,38.6 \mathrm{~Hz}), 101.4(\mathrm{t}, J=$ $1.4 \mathrm{~Hz}), 87.9(\mathrm{t}, J=28.8 \mathrm{~Hz})$

${ }^{19}$ F NMR $(\delta, \mathrm{ppm}) \quad-86.1(\mathrm{~s}),-112.5(\mathrm{~m})$

$\left(282 \mathrm{MHz}, \mathrm{CDCl}_{3}\right)$

IR

$\left(\mathrm{cm}^{-1}, \mathrm{CCl}_{4}\right)$

MS

(HRMS EI)
3068, 3036, 2959, 2928, 2251, 1966, 1497, 1461, 1411, 1340, 1316, $1297,1265,1209,1172,1116,1019$

Calcd for $\mathrm{C}_{11} \mathrm{H}_{7} \mathrm{~F}_{5}: 234.0468$

Found : 234.0467 


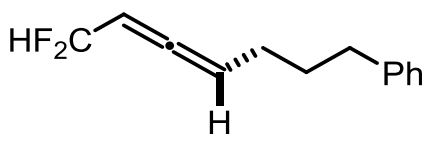

$$
\begin{gathered}
\mathrm{C}_{13} \mathrm{H}_{14} \mathrm{~F}_{2} \\
\mathrm{M}=208.25 \mathrm{~g} \cdot \mathrm{mol}^{-1}
\end{gathered}
$$

Following general procedure E starting with $20(0.20 \mathrm{mmol}$, reaction time : $1 \mathrm{~h})$.

Flash chromatography : pentane

Yield : $29.5 \mathrm{mg}$ (75\%) of a pale yellow oil (crude NMR yield 79\%).

${ }^{1}$ H NMR $(\delta, \mathrm{ppm}) \quad 7.32-7.28(\mathrm{~m}, 2 \mathrm{H}), 7.23-7.17(\mathrm{~m}, 3 \mathrm{H}), 6.10(\mathrm{td}, J=56.6,6.2 \mathrm{~Hz}$, $\left.\left(400 \mathrm{MHz}, \mathrm{CDCl}_{3}\right) \quad 1 \mathrm{H}\right), 5.55(\mathrm{hex}, J=7.0 \mathrm{~Hz}, 1 \mathrm{H}), 5.40(\mathrm{~m}, 1 \mathrm{H}), 2.67(\mathrm{~m}, 2 \mathrm{H}), 2.13$ (qd, $J=7.2,2.0 \mathrm{~Hz}, 2 \mathrm{H}), 1.78(\mathrm{p}, J=7.5 \mathrm{~Hz}, 2 \mathrm{H})$

${ }^{13}$ C NMR $(\delta, \mathrm{ppm}) \quad 206.2(\mathrm{t}, J=12.2 \mathrm{~Hz}), 141.9,128.6,128.5,126.0,114.6(\mathrm{t}, J=237.1$

$\left.\left(101 \mathrm{MHz}, \mathrm{CDCl}_{3}\right) \quad \mathrm{Hz}\right), 96.0 \mathrm{~Hz}(\mathrm{t}, J=1.8 \mathrm{~Hz}), 88.7(\mathrm{t}, J=28.8 \mathrm{~Hz}), 35.3,30.5,27.4(\mathrm{t}$, $J=2.8 \mathrm{~Hz})$

${ }^{19}$ F NMR $(\delta, \mathrm{ppm}) \quad-108.0(\mathrm{~d}, J=56.6 \mathrm{~Hz})$

$\left(282 \mathrm{MHz}, \mathrm{CDCl}_{3}\right)$

IR

3066, 3029, 2930, 2859, 1973, 1604, 1497, 1439, 1354, 1133, 1102,

$\left(\mathrm{cm}^{-1}, \mathrm{CCl}_{4}\right)$

$1060,1031,871$

MS

Calcd for $\mathrm{C}_{13} \mathrm{H}_{14} \mathrm{~F}_{2}: 208.1064$

Found : 208.1053

(HRMS EI)

(3-(benzyloxy)-4,4,4-trifluoro-3-methylbut-1-yn-1-yl)benzene (4p)<smiles>CC([PH2+])=C=CC(F)(F)F</smiles>

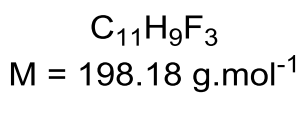

Following general procedure E starting with $\mathbf{2 p}(0.20 \mathrm{mmol}$, reaction time : $1 \mathrm{~h})$.

Flash chromatography : pentane

Yield : $36.0 \mathrm{mg}(91 \%)$ of a colorless oil.

${ }^{1} \mathbf{H}$ NMR $(\delta, \mathrm{ppm}) \quad 7.38-7.25(\mathrm{~m}, 5 \mathrm{H}), 6.56-6.50(\mathrm{~m}, 1 \mathrm{H}), 1.97(\mathrm{~d}, J=3.0 \mathrm{~Hz}, 3 \mathrm{H})$ $\left(300 \mathrm{MHz}, \mathrm{CDCl}_{3}\right)$ 
${ }^{13}$ C NMR $(\delta, \mathrm{ppm}) \quad 204.1(\mathrm{q}, J=3.9 \mathrm{~Hz}), 132.1,129.0,128.3,127.5,123.6(\mathrm{q}, J=273.8$ $\left.\left(75 \mathrm{MHz}, \mathrm{CDCl}_{3}\right) \quad \mathrm{Hz}\right), 100.2,98.0(\mathrm{q}, J=35.2 \mathrm{~Hz}), 13.2$

${ }^{19}$ F NMR $(\delta, \mathrm{ppm}) \quad-66.5$

$\left(282 \mathrm{MHz}, \mathrm{CDCl}_{3}\right)$

IR

$\left(\mathrm{cm}^{-1}\right.$, neat $)$

$2922,2853,2366,2355,2350,1661,1634,1456,1375,1275,1261$, 1117

MS

Calcd for $\left[M+\mathrm{H}^{+}\right] \mathrm{C}_{11} \mathrm{H}_{10} \mathrm{~F}_{3}: 199.0729$

Found : 199.0729

(HRMS APCI)

(6-(trifluoromethyl)deca-4,5-dienyl)benzene (4q)<smiles>CCCC(=C=CCCc1ccccc1)C(F)(F)F</smiles>

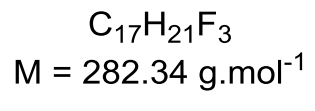

Following general procedure E starting with $\mathbf{2 q}(0.20 \mathrm{mmol}$, reaction time : $1 \mathrm{~h})$.

Flash chromatography : PE

Yield : $53.6 \mathrm{mg}(95 \%)$ of a colorless oil (crude NMR yield $98 \%$ ).

${ }^{1} \mathbf{H}$ NMR $(\delta, \mathrm{ppm}) \quad 7.34-7.28(\mathrm{~m}, 2 \mathrm{H}), 7.24-7.18(\mathrm{~m}, 3 \mathrm{H}), 5.68-5.61(\mathrm{~m}, 1 \mathrm{H}), 2.69(\mathrm{t}$, $\left.\left(400 \mathrm{MHz}, \mathrm{CDCl}_{3}\right) \quad J=7.7 \mathrm{~Hz}, 2 \mathrm{H}\right), 2.18-2.11(\mathrm{~m}, 4 \mathrm{H}), 1.79(\mathrm{p}, J=7.4 \mathrm{~Hz}, 2 \mathrm{H}), 1.55-$ $1.35(\mathrm{~m}, 4 \mathrm{H}), 0.94(\mathrm{t}, J=7.2 \mathrm{~Hz}, 3 \mathrm{H})$

${ }^{13}$ C NMR $(\delta, \mathrm{ppm}) \quad 202.4(\mathrm{q}, J=4.3 \mathrm{~Hz}), 142.1,128.6,128.5,126.0,124.2(\mathrm{q}, J=273.3$ $\left.\left(101 \mathrm{MHz}, \mathrm{CDCl}_{3}\right) \quad \mathrm{Hz}\right), 99.1(\mathrm{q}, J=33.4 \mathrm{~Hz}), 98.2,35.1,30.3,29.6,27.6(\mathrm{~d}, J=1.4 \mathrm{~Hz})$, 26.0, 22.1, 13.8

${ }^{19} \mathbf{F}$ NMR $(\delta, \mathrm{ppm}) \quad-65.2$

$\left(282 \mathrm{MHz}, \mathrm{CDCl}_{3}\right)$

IR 3086, 3065, 3029, 2960, 2934, 2863, 2252, 1976, 1603, 1496, 1455, $\left(\mathrm{cm}^{-1}, \mathrm{CCl}_{4}\right)$ $1292,1253,1292,1253,1156,1121$

MS Calcd for $\mathrm{C}_{17} \mathrm{H}_{21} \mathrm{~F}_{3}: 282.1595$

Found : 282.1584

(HRMS EI) 


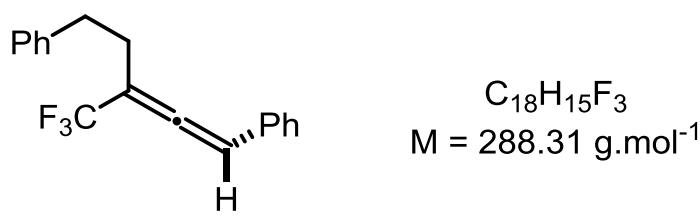

Following general procedure E starting with 2 r $(0.20 \mathrm{mmol}$, reaction time : $10 \mathrm{~h})$.

Flash chromatography : PE

Yield : $45.7 \mathrm{mg}(79 \%)$ of a colorless oil (crude NMR yield $81 \%$ )

${ }^{1} \mathbf{H}$ NMR $(\delta, \mathrm{ppm}) \quad 7.37-7.27(\mathrm{~m}, 5 \mathrm{H}), 7.25-7.19(\mathrm{~m}, 5 \mathrm{H}), 6.61-6.57(\mathrm{~m}, 1 \mathrm{H}), 2.95-$ $\left(400 \mathrm{MHz}, \mathrm{CDCl}_{3}\right) \quad 2.81(\mathrm{~m}, 2 \mathrm{H}), 2.73-2.58(\mathrm{~m}, 2 \mathrm{H})$

${ }^{13}$ C NMR $(\delta, \mathrm{ppm}) \quad 204.2(\mathrm{q}, J=4.1 \mathrm{~Hz}), 140.6,132.0,129.0,128.6,128.6,128.3,127.5$, $\left(101 \mathrm{MHz}, \mathrm{CDCl}_{3}\right) \quad 126.3,123.7(\mathrm{q}, J=274.3 \mathrm{~Hz}), 102.4(\mathrm{q}, J=33.9 \mathrm{~Hz}), 102.0,33.6$, 28.5

${ }^{19}$ F NMR $(\delta$, ppm $) \quad-64.7$

$\left(282 \mathrm{MHz}, \mathrm{CDCl}_{3}\right)$

IR

$3088,3067,3022,2958,2856,2255,1964,1603,1497,1462,1409$,

$\left(\mathrm{cm}^{-1}, \mathrm{CCl}_{4}\right)$ $1299,1282,1261,1214,1152,1124$

MS Calcd for $\mathrm{C}_{18} \mathrm{H}_{15} \mathrm{~F}_{3}: 288.1126$

Found : 288.1123

(HRMS EI)

\section{1-methoxy-3-(5-phenyl-3-(trifluoromethyl)penta-1,2-dienyl)benzene (4s)}

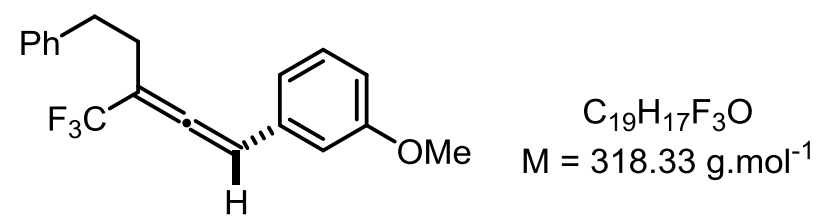

Following general procedure E starting with $2 \mathrm{~s}(0.20 \mathrm{mmol}$, reaction time : $12 \mathrm{~h})$.

Flash chromatography : $\mathrm{PE} / \mathrm{Et}_{2} \mathrm{O} 50: 1$

Yield : $57.8 \mathrm{mg}(91 \%)$ of a colorless oil (crude NMR yield $96 \%$ ).

${ }^{1} \mathbf{H}$ NMR $(\delta, \mathrm{ppm}) \quad 7.27-7.21(\mathrm{~m}, 3 \mathrm{H}), 7.20-7.16(\mathrm{~m}, 3 \mathrm{H}), 6.83-6.79(\mathrm{~m}, 2 \mathrm{H}), 6.76-$ $\left(400 \mathrm{MHz}, \mathrm{CDCl}_{3}\right) \quad 6.75(\mathrm{~m}, 1 \mathrm{H}), 6.53$ (hept, $\left.J=3.3 \mathrm{~Hz}, 1 \mathrm{H}\right), 3.79(\mathrm{~s}, 3 \mathrm{H}), 2.90-2.77$ (m, $2 \mathrm{H}), 2.63-2.57(\mathrm{~m}, 2 \mathrm{H})$ 
${ }^{13}$ C NMR $(\delta, \mathrm{ppm}) \quad 204.2(\mathrm{q}, J=4.1 \mathrm{~Hz}), 160.0,140.6,133.3,130.0,128.6,128.5,126.3$, $\left(101 \mathrm{MHz}, \mathrm{CDCl}_{3}\right) \quad 123.6(\mathrm{q}, J=274.5 \mathrm{~Hz}), 120.1,113.9,112.9,102.5(\mathrm{q}, J=34.0 \mathrm{~Hz})$, $101.9,55.4,33.6,28.4$

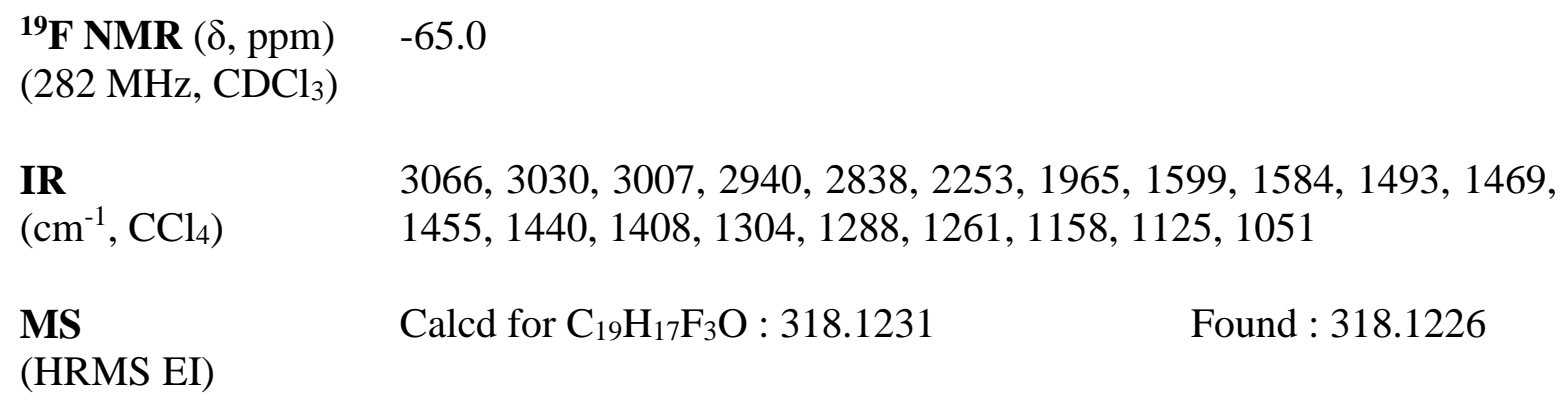

1-bromo-4-(5-phenyl-3-(trifluoromethyl)penta-1,2-dienyl)benzene (4t)

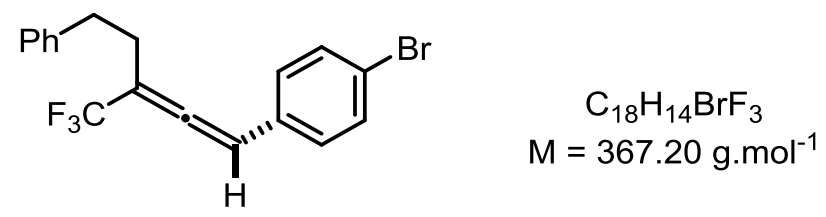

Following general procedure E starting with $2 \mathrm{t}(0.20 \mathrm{mmol}$, reaction time : $8 \mathrm{~h})$.

Flash chromatography : PE

Yield : $69.0 \mathrm{mg}(94 \%)$ of a colorless oil (crude NMR yield $98 \%$ ).

${ }^{1} \mathbf{H}$ NMR $(\delta, \mathrm{ppm}) \quad 7.44-7.40(\mathrm{~m}, 2 \mathrm{H}), 7.29-7.24(\mathrm{~m}, 2 \mathrm{H}), 7.23-7.17(\mathrm{~m}, 3 \mathrm{H}), 7.01-$ $\left(400 \mathrm{MHz}, \mathrm{CDCl}_{3}\right) \quad 6.97(2 \mathrm{H}), 6.52-6.48(\mathrm{~m}, 1 \mathrm{H}), 2.93-2.79(\mathrm{~m}, 2 \mathrm{H}), 2.72-2.56(\mathrm{~m}$, 2H)

${ }^{13}$ C NMR $(\delta, \mathrm{ppm}) \quad 204.3(\mathrm{q}, J=4.1 \mathrm{~Hz}), 140.4,132.1,130.9(\mathrm{q}, J=1.4 \mathrm{~Hz}), 128.9,128.6$, $\left(101 \mathrm{MHz}, \mathrm{CDCl}_{3}\right) \quad 128.6,126.4,123.5(\mathrm{q}, J=274.4 \mathrm{~Hz}), 122.2,102.8(\mathrm{q}, J=34.0 \mathrm{~Hz})$, $101.0,33.4,28.4$

${ }^{19}$ F NMR $(\delta, \mathrm{ppm}) \quad-64.7$

$\left(282 \mathrm{MHz}, \mathrm{CDCl}_{3}\right)$

IR 2928, 2856, 1965, 1604, 1589, 1489, 1454, 1387, 1308, 1293, 1261, $\left(\mathrm{cm}^{-1}, \mathrm{CCl}_{4}\right) \quad 1154,1126$

MS Calcd for $\mathrm{C}_{18} \mathrm{H}_{14} \mathrm{BrF}_{3}: 366.0231$ Found : 366.0227 (HRMS EI) 


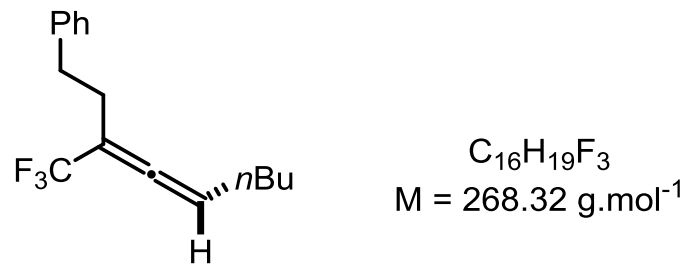

Following general procedure E starting with $\mathbf{2 u}(0.20 \mathrm{mmol}$, reaction time : $4 \mathrm{~h})$.

Flash chromatography : PE

Yield : $53.1 \mathrm{mg}(80 \%)$ of a colorless oil (crude NMR yield $83 \%$ ).

${ }^{1} \mathbf{H}$ NMR $(\delta, \mathrm{ppm}) \quad 7.34-7.28(\mathrm{~m}, 2 \mathrm{H}), 7.25-7.17(\mathrm{~m}, 3 \mathrm{H}), 5.62-5.54(\mathrm{~m}, 1 \mathrm{H}), 2.79(\mathrm{t}$, $\left.\left(400 \mathrm{MHz}, \mathrm{CDCl}_{3}\right) \quad J=7.4 \mathrm{~Hz}, 2 \mathrm{H}\right), 2.53-2.39(\mathrm{~m}, 2 \mathrm{H}), 2.06-1.98(\mathrm{~m}, 2 \mathrm{H}), 1.41-1.33$ $(\mathrm{m}, 4 \mathrm{H}), 0.91(\mathrm{t}, J=6.9 \mathrm{~Hz}, 3 \mathrm{H})$

${ }^{13}$ C NMR $(\delta, \mathrm{ppm}) \quad 202.6(\mathrm{q}, J=4.2 \mathrm{~Hz}), 141.0,128.6,128.5,126.3,124.2(\mathrm{q}, J=273.4$

$\left.\left(101 \mathrm{MHz}, \mathrm{CDCl}_{3}\right) \quad \mathrm{Hz}\right), 99.1,98.2(\mathrm{q}, J=33.7 \mathrm{~Hz}), 33.8,30.9,28.1,27.9,22.2,13.9$

${ }^{19} \mathbf{F}$ NMR $(\delta, \mathrm{ppm}) \quad-65.1$

$\left(282 \mathrm{MHz}, \mathrm{CDCl}_{3}\right)$

IR 3088, 3030, 2961, 2931, 2862, 2255, 1976, 1604, 1497, 1455, 1305, $\left(\mathrm{cm}^{-1}, \mathrm{CCl}_{4}\right)$ $1261,1156,1119,1054,1030$

MS Calcd for $\mathrm{C}_{16} \mathrm{H}_{19} \mathrm{~F}_{3}: 268.1439$

Found : 268.1423

(HRMS EI)

(3-(perfluoroethyl)nona-3,4-dienyl)benzene (4v)<smiles>CCCCC=C=C(CCc1ccccc1)C(C(F)(F)F)C(F)(F)F</smiles>

Following general procedure E starting with $\mathbf{2 v}(0.20 \mathrm{mmol}$, reaction time : $23 \mathrm{~h})$.

Flash chromatography : PE

Yield : $51.6 \mathrm{mg}(80 \%)$ of a colorless oil (crude NMR yield $88 \%$ ) 


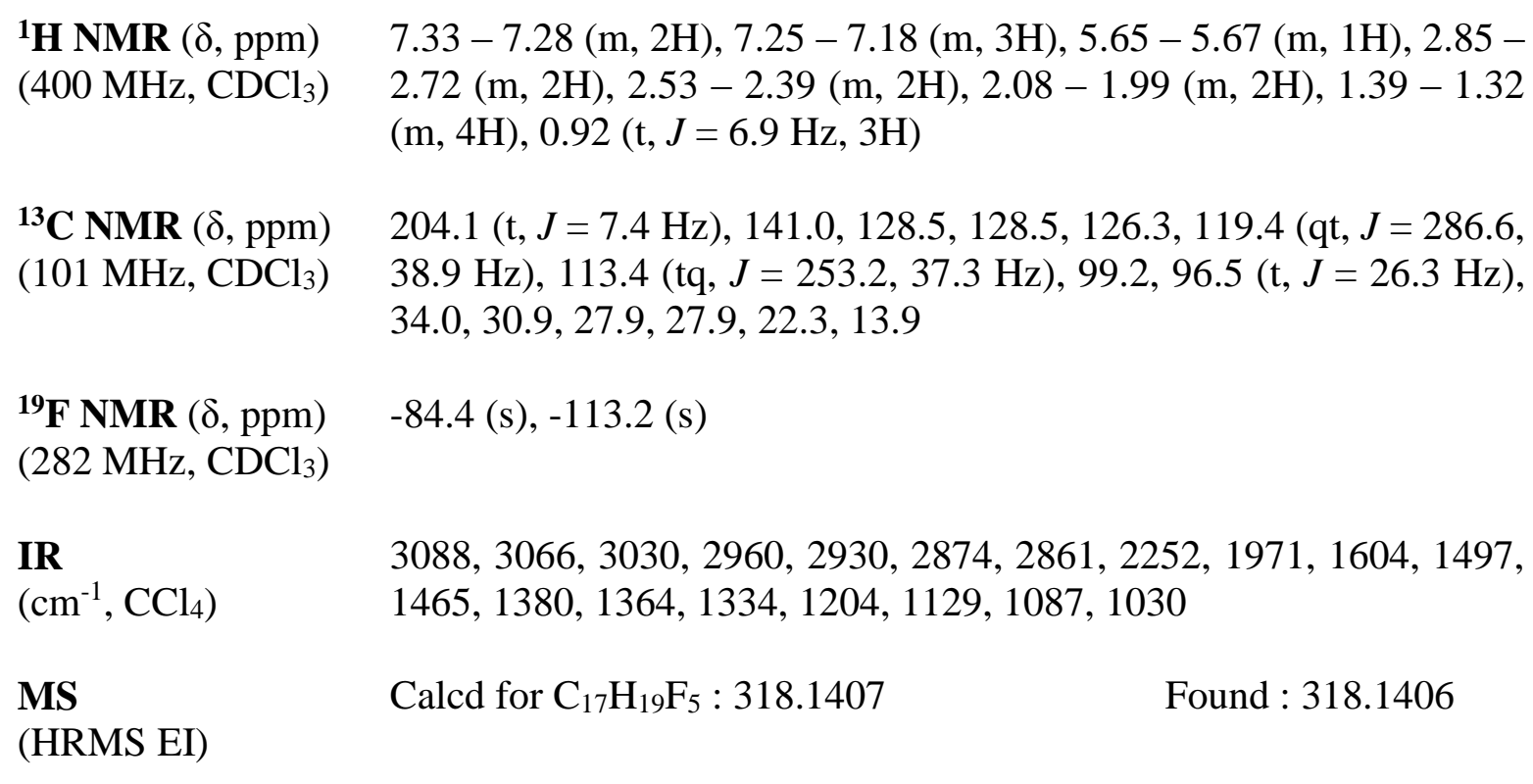

(1,1,1-trifluoronona-2,3-dien-2-yl)benzene (4w) \& 1-pentyl-3-(trifluoromethyl)-1H-indene $(8 w)$

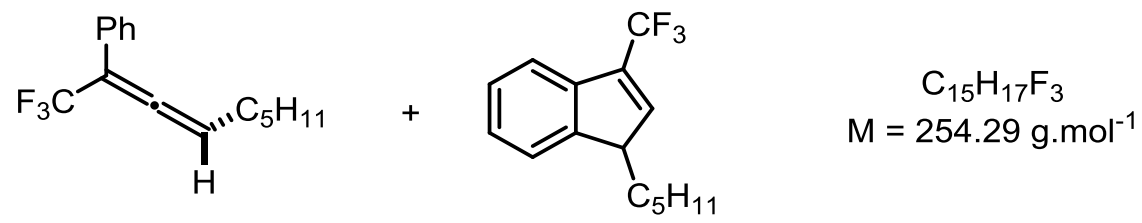

Following general procedure E starting with $\mathbf{2 w}(0.1 \mathrm{mmol})$.

Flash chromatography : PE

Yield : Using catalyst 6 at $60{ }^{\circ} \mathrm{C}$ during $16 \mathrm{~h}$ led to the formation of a mixture of $18 \%$ of allene and $81 \%$ of indene (NMR yields). Using catalyst $7 \mathbf{a}$ at room temperature during $24 \mathrm{~h}$ led to an isolated yield of $81 \%$ (mixture allene/indene $5: 1$ )

Characterization: Due to the difficulty to separate an allene from the corresponding indene, they were characterized together. A pure sample of the indene could be obtained by adding an excess of gold catalyst to an allene/indene mixture until complete conversion of the allene into the indene. A sample of the allene could be obtained by adding an excess of $\mathrm{NaH}$ to an allene/indene mixture (in order to deprotonate the indene) and by quenching it with an electrophile (benzaldehyde). In both cases, purification by flash chromatography with pure PE was carried out.

\section{Allene :}

${ }^{1} \mathbf{H}$ NMR $(\delta, \mathrm{ppm}) \quad 7.45-7.42(\mathrm{~m}, 2 \mathrm{H}), 7.39-7.34(\mathrm{~m}, 2 \mathrm{H}), 7.31-7.28(\mathrm{~m}, 1 \mathrm{H}), 5.95$ $\left(400 \mathrm{MHz}, \mathrm{CDCl}_{3}\right) \quad(\mathrm{tq}, J=6.6 \mathrm{~Hz}, 3.2 \mathrm{~Hz}, 1 \mathrm{H}), 2.25-2.19(\mathrm{~m}, 2 \mathrm{H}), 1.54-1.48(\mathrm{~m}, 2 \mathrm{H})$, $1.39-1.25(\mathrm{~m}, 4 \mathrm{H}), 0.90-0.86(\mathrm{~m}, 3 \mathrm{H})$ 


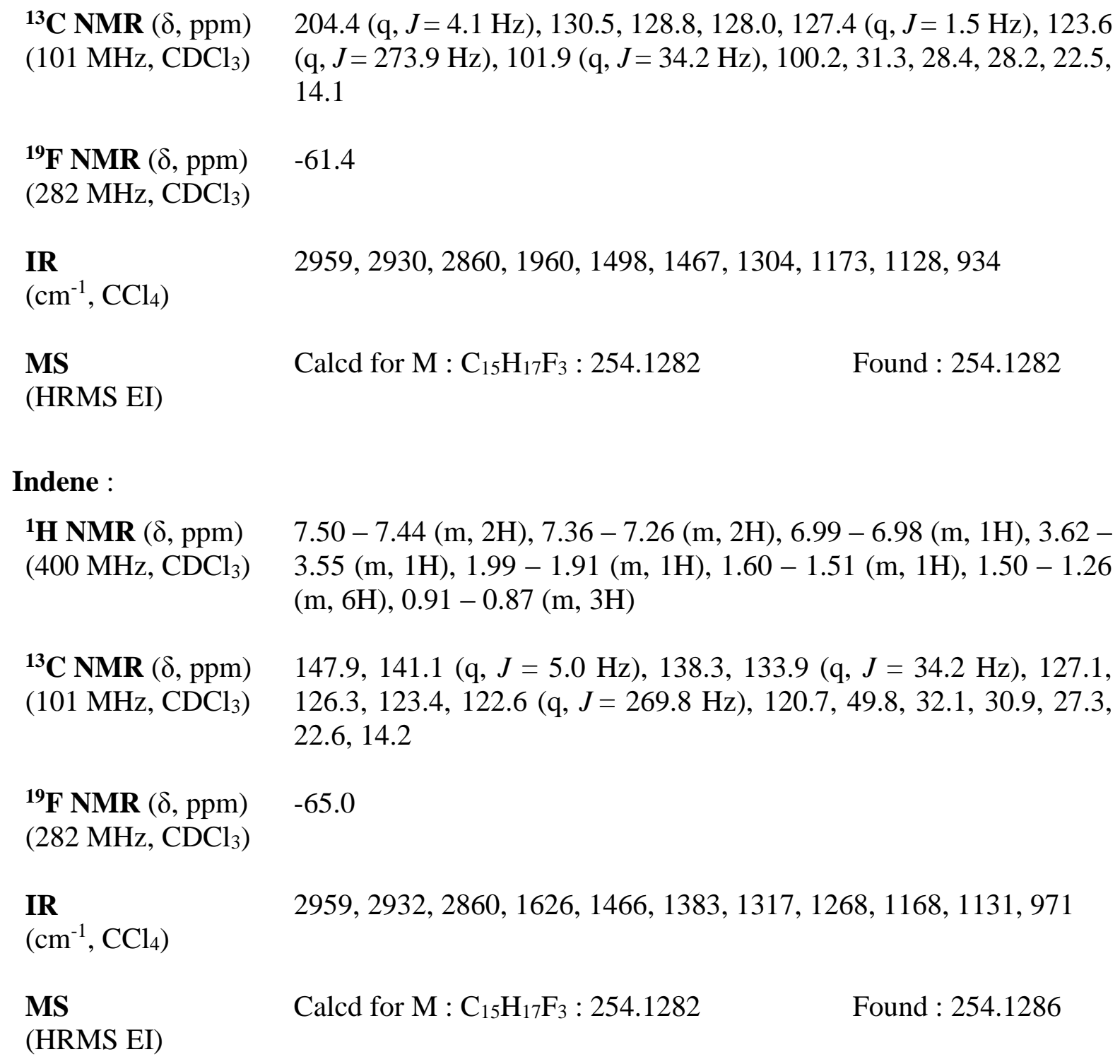

(1,1,1-trifluoronona-2,3-dien-2-yl)benzene (4x) \& 1-pentyl-3-(trifluoromethyl)-1H-indene $(8 x)$

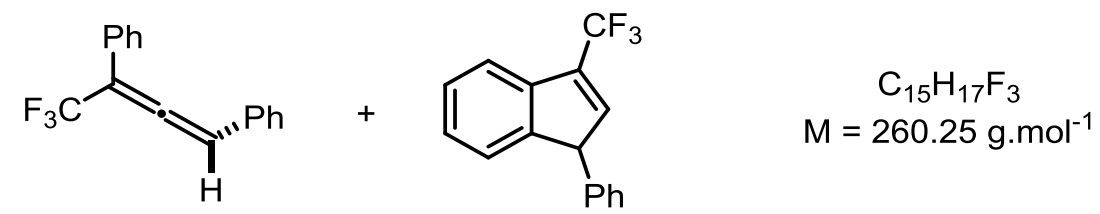

Following general procedure E starting with $2 \mathbf{x}(0.2 \mathrm{mmol})$.

Due to the instability of the indene on silica, the mixture could not be purified. NMR yields could be obtained by using characteristic signals.

Yield : Using catalyst $7 \mathbf{a}$ at $60{ }^{\circ} \mathrm{C}$ during $20 \mathrm{~h}$ led to the formation of a mixture of $21 \%$ of allene and $72 \%$ of indene (NMR yields). 
${ }^{1} \mathbf{H}$ NMR $(\delta, \mathrm{ppm}) \quad$ Characteristic peaks : $6.94(\mathrm{q}, J=3.1 \mathrm{~Hz}, 1 \mathrm{H}$, allene), $4.74-4.71(\mathrm{~m}$, $\left(300 \mathrm{MHz}, \mathrm{CDCl}_{3}\right) \quad 1 \mathrm{H}$, indene)

${ }^{19}$ F NMR $(\delta, \mathrm{ppm}) \quad-61.0$ (allene), -65.0 (indene)

$\left(282 \mathrm{MHz}, \mathrm{CDCl}_{3}\right)$

(3-(benzyloxy)-4,4,4-trifluoro-3-methylbut-1-yn-1-yl)benzene (9y)

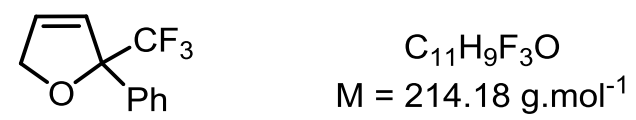

Following general procedure E starting with $2 \mathbf{y}(0.20 \mathrm{mmol}$, reaction time : $1 \mathrm{~h})$.

Flash chromatography : PE/EtOAc $98: 2$

Yield : $35.2 \mathrm{mg}(82 \%)$ of a colorless oil.

${ }^{1} \mathbf{H}$ NMR $(\delta, \mathrm{ppm}) \quad 7.55-7.53(\mathrm{~m}, 2 \mathrm{H}), 7.43-7.33(\mathrm{~m}, 3 \mathrm{H}), 6.25(\mathrm{~d}, J=6.1 \mathrm{~Hz}, 1 \mathrm{H})$, $\left(300 \mathrm{MHz}, \mathrm{CDCl}_{3}\right) \quad 6.16(\mathrm{dt}, J=6.1,2.4 \mathrm{~Hz}, 1 \mathrm{H}), 4.97(\mathrm{ddd}, J=13.4,2.4,1.6 \mathrm{~Hz}, 1 \mathrm{H})$, $4.85-4.81(\mathrm{~d}, J=13.4 \mathrm{~Hz}, 1 \mathrm{H})$

${ }^{13}$ C NMR $(\delta, \mathrm{ppm}) \quad 137.1,131.7,128.7,128.5,126.4,125.2,124.8(\mathrm{q}, J=285.9 \mathrm{~Hz}), 92.2$ $\left(75 \mathrm{MHz}, \mathrm{CDCl}_{3}\right) \quad(\mathrm{q}, J=30.2 \mathrm{~Hz}), 77.1$

${ }^{19} \mathbf{F}$ NMR $(\delta, \mathrm{ppm}) \quad-79.4$

$\left(282 \mathrm{MHz}, \mathrm{CDCl}_{3}\right)$

IR

$\left(\mathrm{cm}^{-1}\right.$, neat $)$

MS

(HRMS APCI)
2957, 2924, 2851, 2357, 1647, 1458, 1377, 1275, 1180, 1132, 1078, 945

Calcd for $\left[M-\mathrm{CF}_{3}{ }^{+}\right] \mathrm{C}_{10} \mathrm{H}_{9} \mathrm{O}+: 145.0648$

Found : 145.0648

5-isopropyl-2-phenyl-2-(trifluoromethyl)-2,5-dihydrofuran $(9 z)$

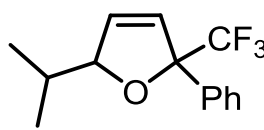

$$
\begin{gathered}
\mathrm{C}_{14} \mathrm{H}_{15} \mathrm{~F}_{3} \mathrm{O} \\
\mathrm{M}=256.26 \mathrm{~g} \cdot \mathrm{mol}^{-1}
\end{gathered}
$$

Following general procedure E starting with $\mathbf{2 z}(0.20 \mathrm{mmol}$, reaction time : $2 \mathrm{~h})$.

Flash chromatography : PE/EtOAc $99: 1$

Yield : $45.1 \mathrm{mg}(88 \%)$ of a colorless oil as a $1: 1$ mixture of two diastereomers. 
The diastereomers were separated by HPLC using a XBridge C18 10x100mm 5 $\mu \mathrm{m}$ column $\left(\mathrm{H}_{2} \mathrm{O} / \mathrm{CH}_{3} \mathrm{CN} 40 / 60,5 \mathrm{~mL} / \mathrm{min}, 210 \mathrm{~nm}\right.$, $\left.\mathrm{tR}_{\text {trans }}=3.999 \mathrm{~min}, \mathrm{tR}_{\mathrm{cis}}=4.664 \mathrm{~min}\right)$.

trans-5-isopropyl-2-phenyl-2-(trifluoromethyl)-2,5-dihydrofuran (trans-9z)

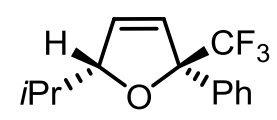

Chemical Formula: $\mathrm{C}_{14} \mathrm{H}_{15} \mathrm{~F}_{3} \mathrm{O}$

Molecular Weight: 256,27

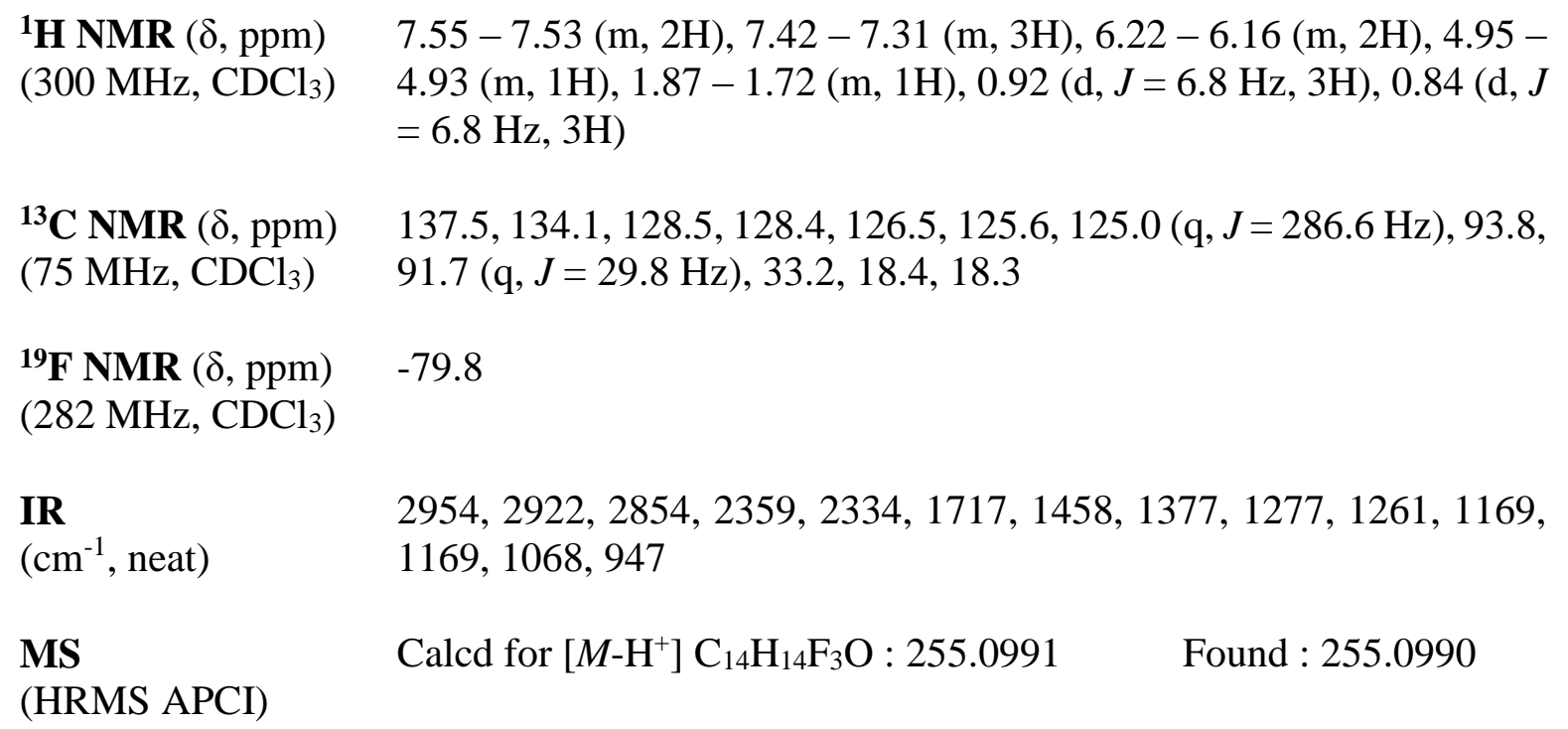

The stereochemistry was determined by H-H NOESY correlation. The NOESY spectrum showed NOE between $\mathrm{H}_{\mathrm{b}}$ and $\mathrm{H}_{\mathrm{d}}$. Furthermore, no NOE between $\mathrm{H}_{\mathrm{a}}$ and $\mathrm{H}_{\mathrm{b}}$ was observed. This product was assigned to the trans-isomer.

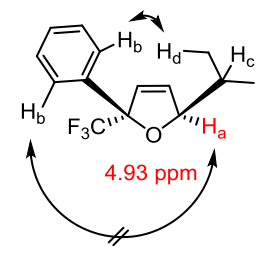

cis-5-isopropyl-2-phenyl-2-(trifluoromethyl)-2,5-dihydrofuran (cis-9z)<smiles>CC(I)[C@H]1C=C[C@](c2ccccc2)([PH+](F)(F)(F)(F)F)O1</smiles> 


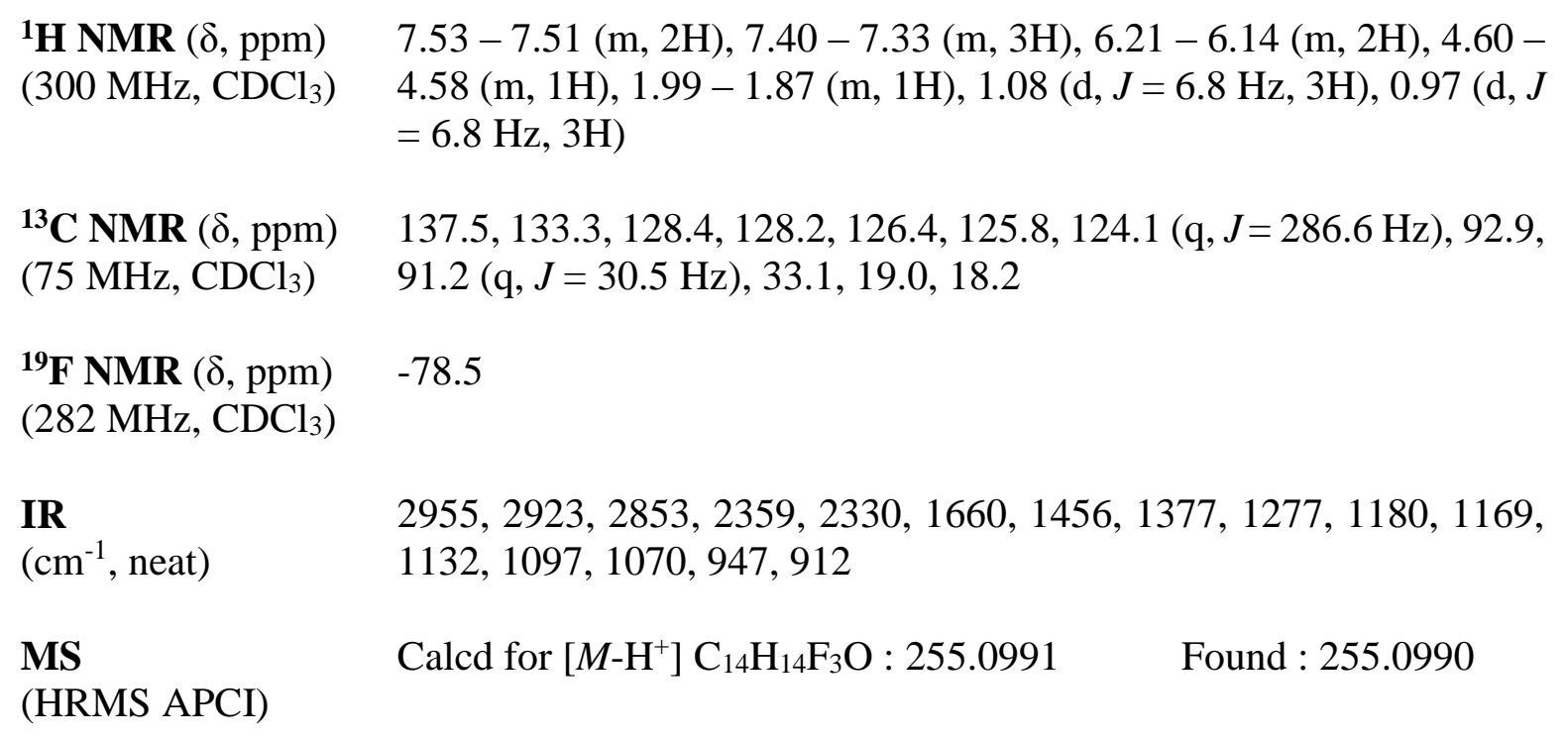

The stereochemistry was determined by H-H NOESY correlation. The NOESY spectrum showed NOE between $\mathrm{H}_{\mathrm{a}}$ and $\mathrm{H}_{\mathrm{b}}$. Furthermore, no NOE between $\mathrm{H}_{\mathrm{b}}$ and $\mathrm{H}_{\mathrm{d}}$ was observed. This product was assigned to the $c i s$-isomer.

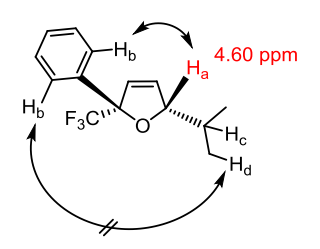

\section{Post-functionalization of the $\mathrm{CF}_{3}$-allenes}

\section{Palladium-catalyzed diboration}

This reaction was carried out inspired from a literature procedure. ${ }^{5} \mathrm{Pd}(\mathrm{dba})_{2}(0.05 \mathrm{eq}, 0.005$ mmol, $2.9 \mathrm{mg}$ ) and $\mathrm{P}\left(\mathrm{NMe}_{2}\right)_{3}$ (stock solution $0.06 \mathrm{~mol}^{-\mathrm{L}^{-1}}$ in toluene) (0.06 eq, $0.006 \mathrm{mmol}$, $0.1 \mathrm{~mL}$ of solution) were dissolved in toluene $(0.9 \mathrm{~mL})$. After $30 \mathrm{~min}$ of stirring at room temperature, bispinacolatodiboron (1.4 eq, $0.14 \mathrm{mmol}, 35.6 \mathrm{mg}$ ) was added, followed by allene $4 \mathbf{k}$ (1.0 eq, $0.10 \mathrm{mmol}, 22.6 \mathrm{mg})$ dissolved in toluene $(0.25 \mathrm{~mL})$. The mixture was stirred for 5 $\mathrm{h}$ at room temperature and filtered on silica (eluting with $\mathrm{Et}_{2} \mathrm{O}$ ). The crude mixture was purified by flash column chromatography $\left(\mathrm{PE} \mathrm{Et}_{2} \mathrm{O}\right.$ 95/5) to furnish the diborated product as two isomers (colourless oil, $29 \mathrm{mg}$, 60\% global yield, Z/E $2: 1$ ratio). Both isomers could be separated by column chromatography and characterized individually. 
dioxaborolane) ((Z)-10) (major isomer)

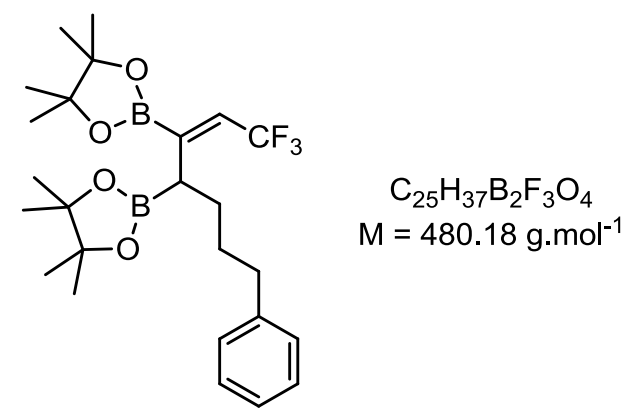

${ }^{1} \mathbf{H}$ NMR $(\delta, \mathrm{ppm}) \quad 7.27-7.22(\mathrm{~m}, 3 \mathrm{H}), 7.16-7.12(\mathrm{~m}, 2 \mathrm{H}), 6.24(\mathrm{q}, J=8.8 \mathrm{~Hz}, 1 \mathrm{H})$, $\left(300 \mathrm{MHz}, \mathrm{CDCl}_{3}\right) \quad 2.69-2.45(\mathrm{~m}, 3 \mathrm{H}), 1.80-1.72(\mathrm{~m}, 2 \mathrm{H}), 1.57-1.47(\mathrm{~m}, 2 \mathrm{H}), 1.22-$ $1.20(\mathrm{~m}, 24 \mathrm{H})$

${ }^{13}$ C NMR $(\delta, \mathrm{ppm}) \quad 143.0,128.6,128.3,128.0(\mathrm{q}, J=32.2 \mathrm{~Hz}), 125.6,36.1,31.4,29.1$, $\left(125 \mathrm{MHz}, \mathrm{CDCl}_{3}\right) \quad 25.0,24.9,24.7,24.6$ (carbons next to boron are not observed due to quadrupolar coupling effects of ${ }^{11} \mathrm{~B} ;-\mathrm{CF}_{3}$ carbon is not observed because of the low intensity of the quadruplet)

${ }^{19}$ F NMR $(\delta$, ppm $) \quad-57.8$

$\left(282 \mathrm{MHz}, \mathrm{CDCl}_{3}\right)$

IR

$\left(\mathrm{cm}^{-1}\right.$, neat $)$

MS

(HRMS APCI)
$2978,2928,2854,2359,2330,1684,1636,1558,1541,1506,1456$, $1373,1339,1325,1263,1215,1140,1117,1088,968,864$

Calcd for $\left[M+\mathrm{H}^{+}\right] \mathrm{C}_{25} \mathrm{H}_{38} \mathrm{~B}_{2} \mathrm{~F}_{3} \mathrm{O}_{4}: 481.2904 \quad$ Found : 481.2907

The stereochemistry was determined by H-H NOESY correlation. No significant correlation was observed on this isomer. The NOESY correlations of the other isomer are needed to confirm the stereochemistry of each compound.

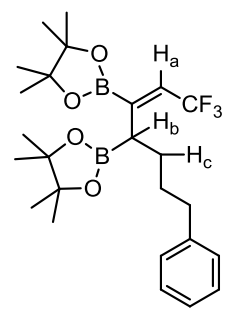

(E)-2,2'-(1,1,1-trifluoro-7-phenylhept-2-ene-3,4-diyl)bis(4,4,5,5-tetramethyl-1,3,2dioxaborolane) ((E)-10) (minor isomer) 


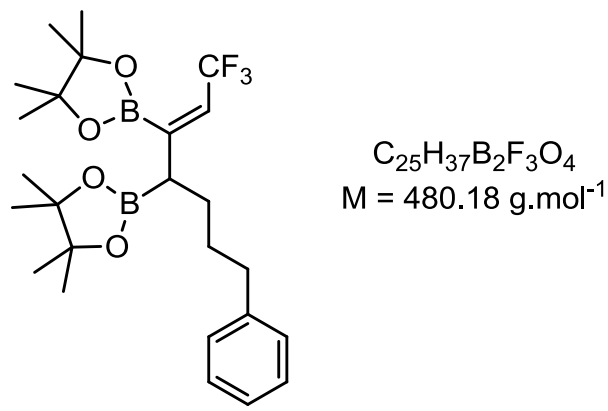
${ }^{1} \mathbf{H}$ NMR $(\delta, \mathrm{ppm}) \quad 7.29-7.24(\mathrm{~m}, 2 \mathrm{H}), 7.19-7.14(3 \mathrm{H}), 5.81(\mathrm{q}, J=7.8 \mathrm{~Hz}, 1 \mathrm{H}), 2.60$ $\left(300 \mathrm{MHz}, \mathrm{CDCl}_{3}\right) \quad(\mathrm{t}, J=6.5 \mathrm{~Hz}, 2 \mathrm{H}), 2.02-1.98(\mathrm{~m}, 1 \mathrm{H}), 1.71-1.56(\mathrm{~m}, 4 \mathrm{H}), 1.27-$ $1.22(\mathrm{~m}, 24 \mathrm{H})$
${ }^{13}$ C NMR $(\delta, \mathrm{ppm}) \quad 142.6,128.6,128.4,125.8,36.0,31.0,29.6,25.0,24.9,24.8,24.8$ $\left(75 \mathrm{MHz}, \mathrm{CDCl}_{3}\right) \quad$ (carbons next to boron are not observed due to quadrupolar coupling effects of ${ }^{11} \mathrm{~B}$; carbons close to fluorine atoms are not observed because of the low intensity of the quadruplets)

${ }^{19} \mathbf{F ~ N M R}(\delta, \mathrm{ppm})$
$\left(282 \mathrm{MHz}, \mathrm{CDCl}_{3}\right)$

IR

$\left(\mathrm{cm}^{-1}\right.$, neat $)$

$2976,2924,2851,2359,2330,1734,1647,1541,1456,1362,1325$, $1292,1275,1132,1117,966,856$

MS

Calcd for $\left[M+\mathrm{H}^{+}\right] \mathrm{C}_{25} \mathrm{H}_{38} \mathrm{~B}_{2} \mathrm{~F}_{3} \mathrm{O}_{4}: 481.2904 \quad$ Found : 481.2907

(HRMS APCI)

The stereochemistry was determined by H-H NOESY correlation. The NOESY spectrum showed NOE between $\mathrm{H}_{\mathrm{b}}$ and $\mathrm{H}_{\mathrm{a}}$. This product was assigned to the (E)-isomer.

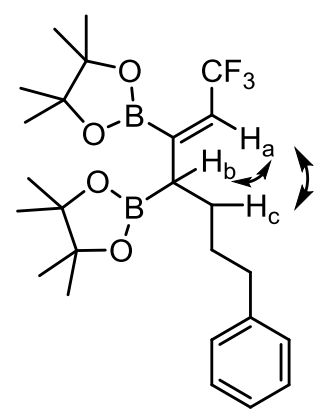

\section{Copper-catalyzed hydroboration}

$\operatorname{IMesCuDBM}(0.05$ eq, $0.005 \mathrm{mmol}, 3.0 \mathrm{mg}$ ) and bispinacolatodiboron (1.1 eq, $0.11 \mathrm{mmol}, 27.9$ $\mathrm{mg})$ were dissolved in THF $(0.5 \mathrm{~mL})$ and stirred for 5 minutes at room temperature. Allene $\mathbf{4 k}$ (1.0 eq, $0.1 \mathrm{mmol}, 22.6 \mathrm{mg}$ ) and $\mathrm{MeOH}(7.4 \mathrm{eq}, 0.74 \mathrm{mmol}, 23.8 \mathrm{mg}, 30 \mu \mathrm{L}$ ) were dissolved in 
THF $(0.2 \mathrm{~mL})$ and add to the reaction. The mixture was stirred for $15 \mathrm{~h}$ at room temperature and filtered on silica (eluting with $\mathrm{Et}_{2} \mathrm{O}$ ). The crude mixture was purified by flash column chromatography (PE/Et $2 \mathrm{O} 97 / 3)$ to furnish the hydroborated product as two isomers (colourless oil, $20.7 \mathrm{mg}$, 58\% global yield, Z/E $6: 1$ ratio). Both isomers could be separated by column chromatography and characterized individually.

(Z)-4,4,5,5-tetramethyl-2-(1,1,1-trifluoro-7-phenylhept-3-en-3-yl)-1,3,2-dioxaborolane ((Z)-11) (major isomer)

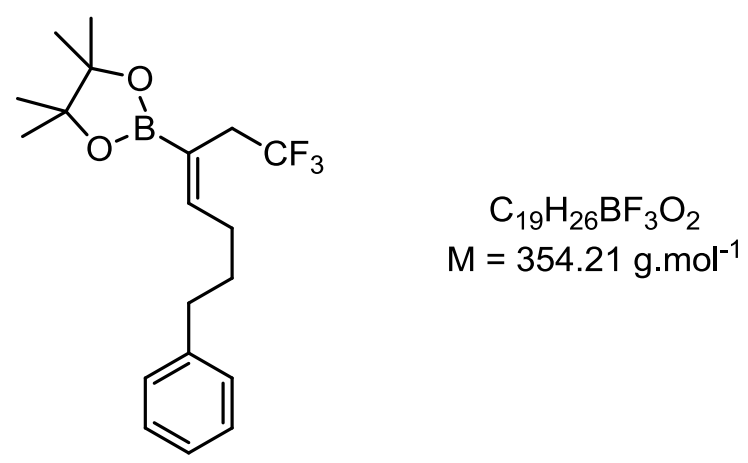

\footnotetext{
${ }^{1} \mathbf{H}$ NMR $(\delta, \mathrm{ppm}) \quad 7.31-7.25(\mathrm{~m}, 2 \mathrm{H}), 7.20-7.16(\mathrm{~m}, 3 \mathrm{H}), 6.63(\mathrm{t}, J=7.1 \mathrm{~Hz}, 1 \mathrm{H}), 2.93$ $\left(300 \mathrm{MHz}, \mathrm{CDCl}_{3}\right) \quad(\mathrm{q}, J=11.0 \mathrm{~Hz}, 2 \mathrm{H}), 2.67-2.62(\mathrm{~m}, 2 \mathrm{H}), 2.26-2.18(\mathrm{~m}, 2 \mathrm{H}), 1.82-$ $1.72(\mathrm{~m}, 2 \mathrm{H}), 1.26(\mathrm{~s}, 12 \mathrm{H})$

${ }^{13}$ C NMR $(\delta, \mathrm{ppm}) \quad 152.4,141.9,128.4,128.4,126.4(\mathrm{q}, J=277.2 \mathrm{~Hz}), 125.8,83.6,35.5$, $\left(75 \mathrm{MHz}, \mathrm{CDCl}_{3}\right) \quad 32.5(\mathrm{q}, J=29.3 \mathrm{~Hz}), 30.2,28.7,24.6$ (carbon next to boron is not observed due to quadrupolar coupling effects of $\left.{ }^{11} \mathrm{~B}\right)$

${ }^{19} \mathbf{F}$ NMR $(\delta, \mathrm{ppm}) \quad-65.8$

$\left(282 \mathrm{MHz}, \mathrm{CDCl}_{3}\right)$

IR

$\left(\mathrm{cm}^{-1}\right.$, neat $)$

2978, 2930, 2856, 2357, 2330, 1734, 1636, 1605, 1497, 1454, 1416,

MS $1381,1346,1315,1277,1252,1215,1167,1117,1030,962,924,864$

(HRMS APCI)

Calcd for $\left[M+\mathrm{Na}^{+}\right] \mathrm{C}_{19} \mathrm{H}_{26} \mathrm{O}_{2} \mathrm{BF}_{3} \mathrm{Na}: 377.1870$ Found : 377.1872
}

The stereochemistry was determined by H-H NOESY correlation. The NOESY spectrum showed NOE between $\mathrm{H}_{\mathrm{a}}$ and $\mathrm{H}_{\mathrm{b}}$ as well as between $\mathrm{H}_{\mathrm{c}}$ and $\mathrm{H}_{\mathrm{d}}$. Furthermore, no NOE between $\mathrm{H}_{\mathrm{a}}$ and $\mathrm{H}_{\mathrm{c}}$ or between $\mathrm{H}_{\mathrm{b}}$ and $\mathrm{H}_{\mathrm{d}}$ was observed. This product was assigned to the $(Z)$-isomer. 


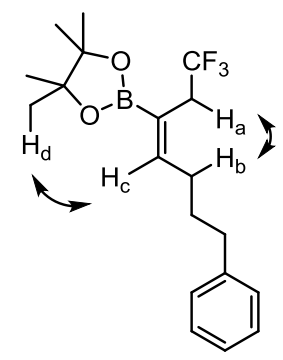

(E)-4,4,5,5-tetramethyl-2-(1,1,1-trifluoro-7-phenylhept-3-en-3-yl)-1,3,2-dioxaborolane ((E)-11) (minor isomer)<smiles>CC1(C)OB(/C(=C/CCCc2ccccc2)CC(F)(F)F)OC1(C)C</smiles>

$$
\begin{gathered}
\mathrm{C}_{19} \mathrm{H}_{26} \mathrm{BF}_{3} \mathrm{O}_{2} \\
M=354.21 \mathrm{~g} \cdot \mathrm{mol}^{-1}
\end{gathered}
$$

${ }^{1} \mathbf{H}$ NMR $(\delta, \mathrm{ppm})$

$7.30-7.25(\mathrm{~m}, 2 \mathrm{H}, 7.19-7.16(\mathrm{~m}, 3 \mathrm{H}), 6.30(\mathrm{t}, J=7.5 \mathrm{~Hz}, 1 \mathrm{H}), 2.85$

$\left(300 \mathrm{MHz}, \mathrm{CDCl}_{3}\right)$

$(\mathrm{q}, J=11.1 \mathrm{~Hz}, 2 \mathrm{H}), 2.64-2.59(\mathrm{~m}, 2 \mathrm{H}), 2.48-2.40(\mathrm{~m}, 2 \mathrm{H}), 1.76-$ $1.46(\mathrm{~m}, 2 \mathrm{H}), 1.23(\mathrm{~s}, 12 \mathrm{H})$

${ }^{13} \mathbf{C ~ N M R}(\delta, \mathrm{ppm}) \quad 154.2,142.6,128.6,128.4,125.8,83.6,40.3(\mathrm{q}, J=28.6 \mathrm{~Hz}), 35.6$, $\left(75 \mathrm{MHz}, \mathrm{CDCl}_{3}\right) \quad 31.4,31.1,24.8$ (carbon next to boron is not observed due to quadrupolar coupling effects of ${ }^{11} \mathrm{~B},-\mathrm{CF}_{3}$ carbon is not observed because of the low intensity of the quadruplet)

${ }^{19}$ F NMR $(\delta, \mathrm{ppm}) \quad-67.0$

$\left(282 \mathrm{MHz}, \mathrm{CDCl}_{3}\right)$

IR

$\left(\mathrm{cm}^{-1}\right.$, neat $)$

MS

(HRMS APCI)
2957, 2922, 2854, 2359, 2324, 1636, 1558, 1541, 1456, 1275, 1259, $1134,1121,1086,959,908,864$

Calcd for $\left[M+\mathrm{H}^{+}\right] \mathrm{C}_{19} \mathrm{H}_{27} \mathrm{O}_{2} \mathrm{BF}_{3}: 355.2051$ 
The stereochemistry was determined by H-H NOESY correlation. The NOESY spectrum showed NOE between $\mathrm{H}_{\mathrm{a}}$ and $\mathrm{H}_{\mathrm{c}}$ as well as between $\mathrm{H}_{\mathrm{b}}$ and $\mathrm{H}_{\mathrm{d}}$. Furthermore, no NOE between $\mathrm{H}_{\mathrm{a}}$ and $\mathrm{H}_{\mathrm{b}}$ or between $\mathrm{H}_{\mathrm{c}}$ and $\mathrm{H}_{\mathrm{d}}$ was observed. This product was assigned to the $(E)$-isomer.

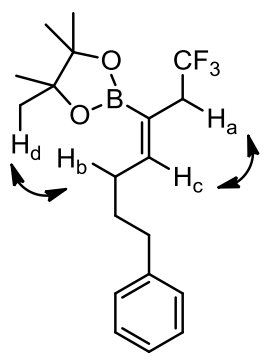




\section{NMR analyses}
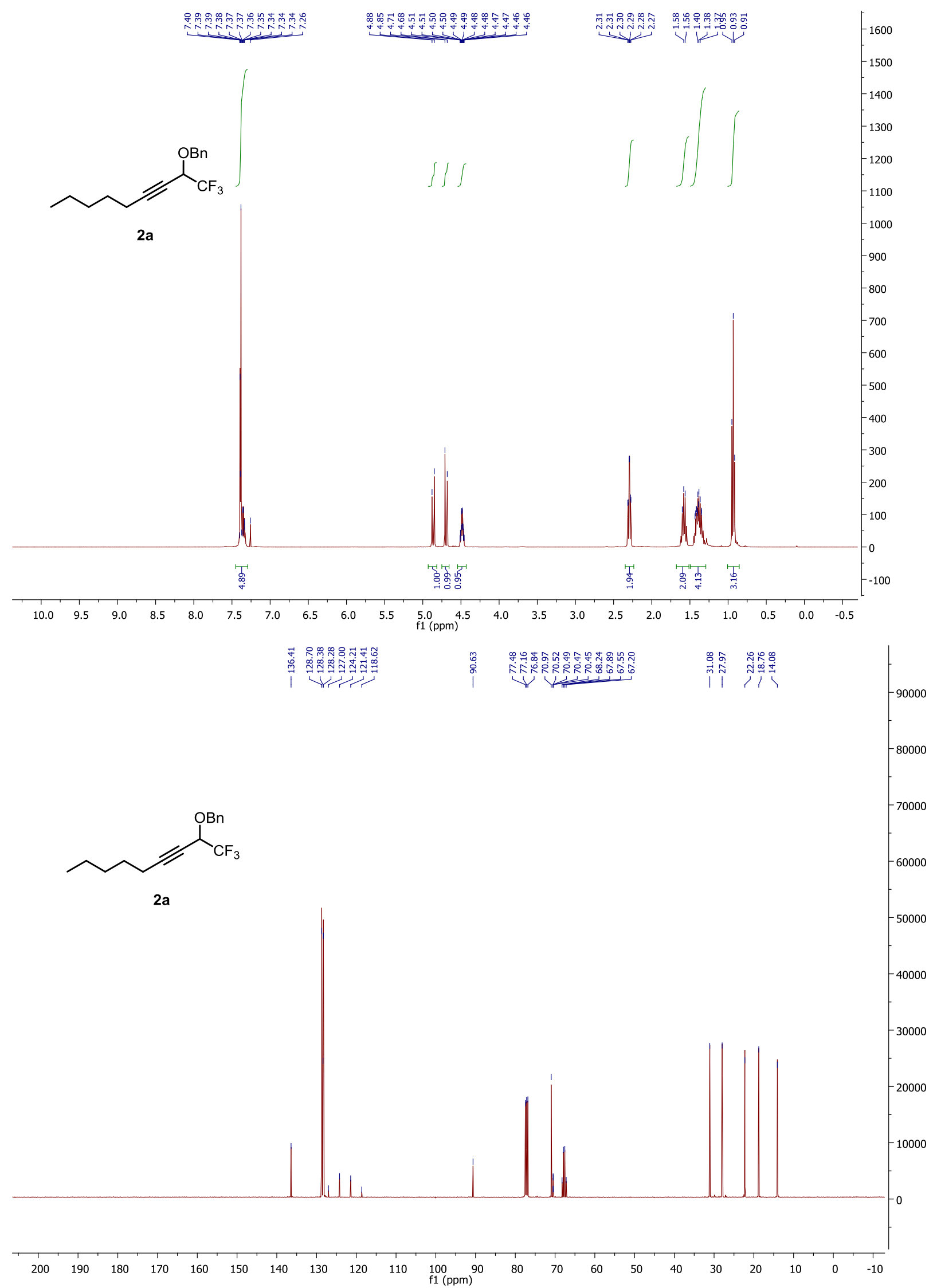


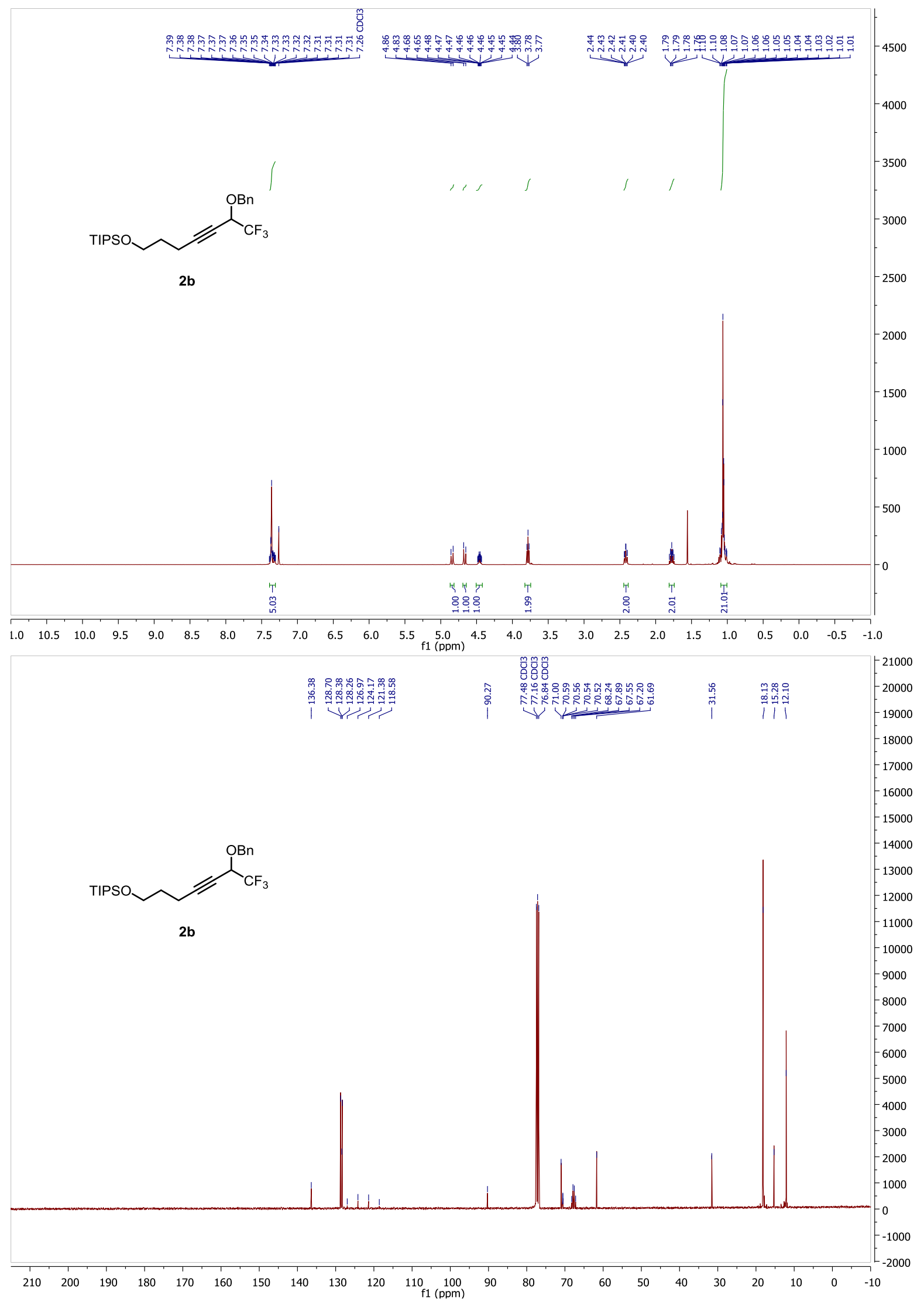



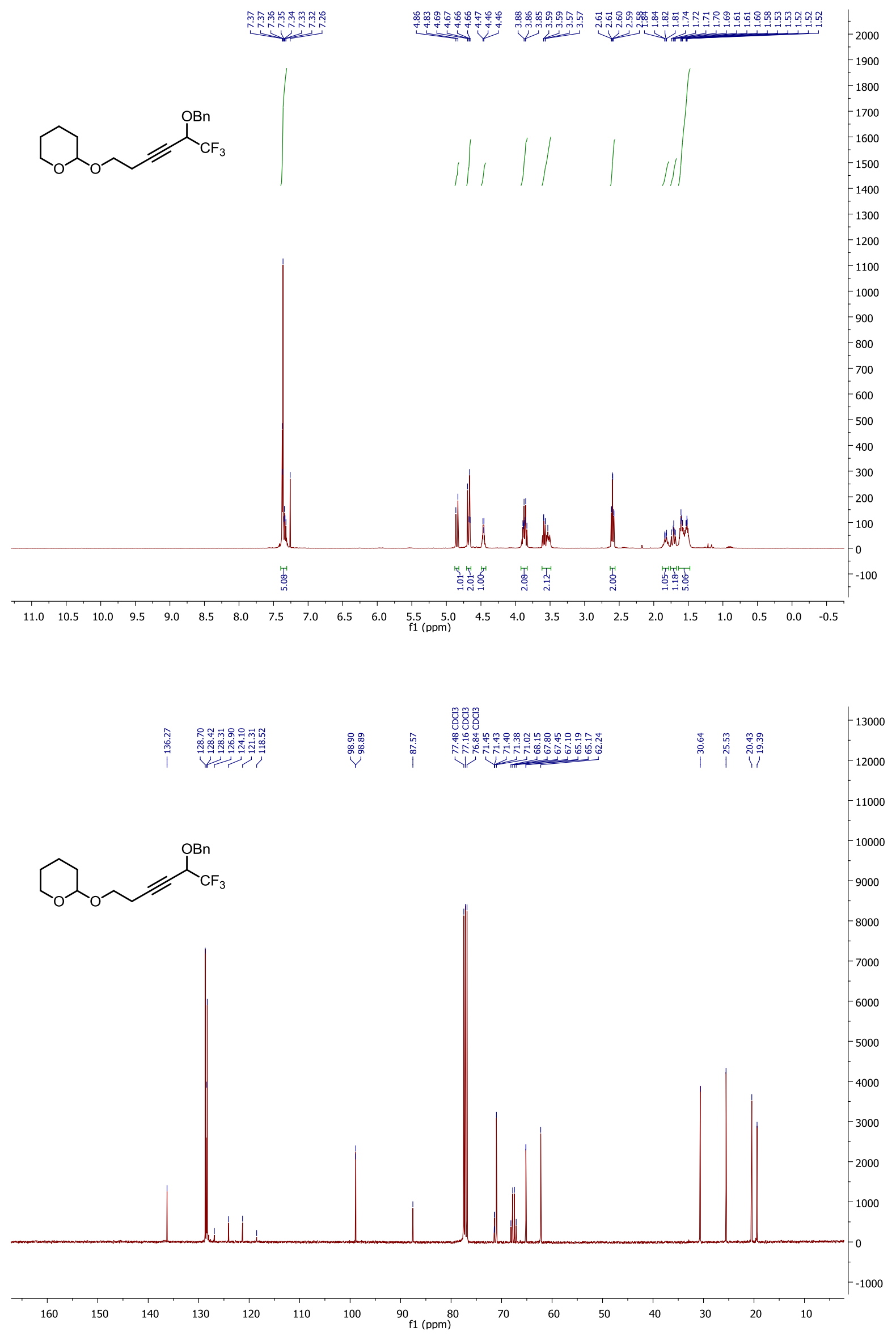

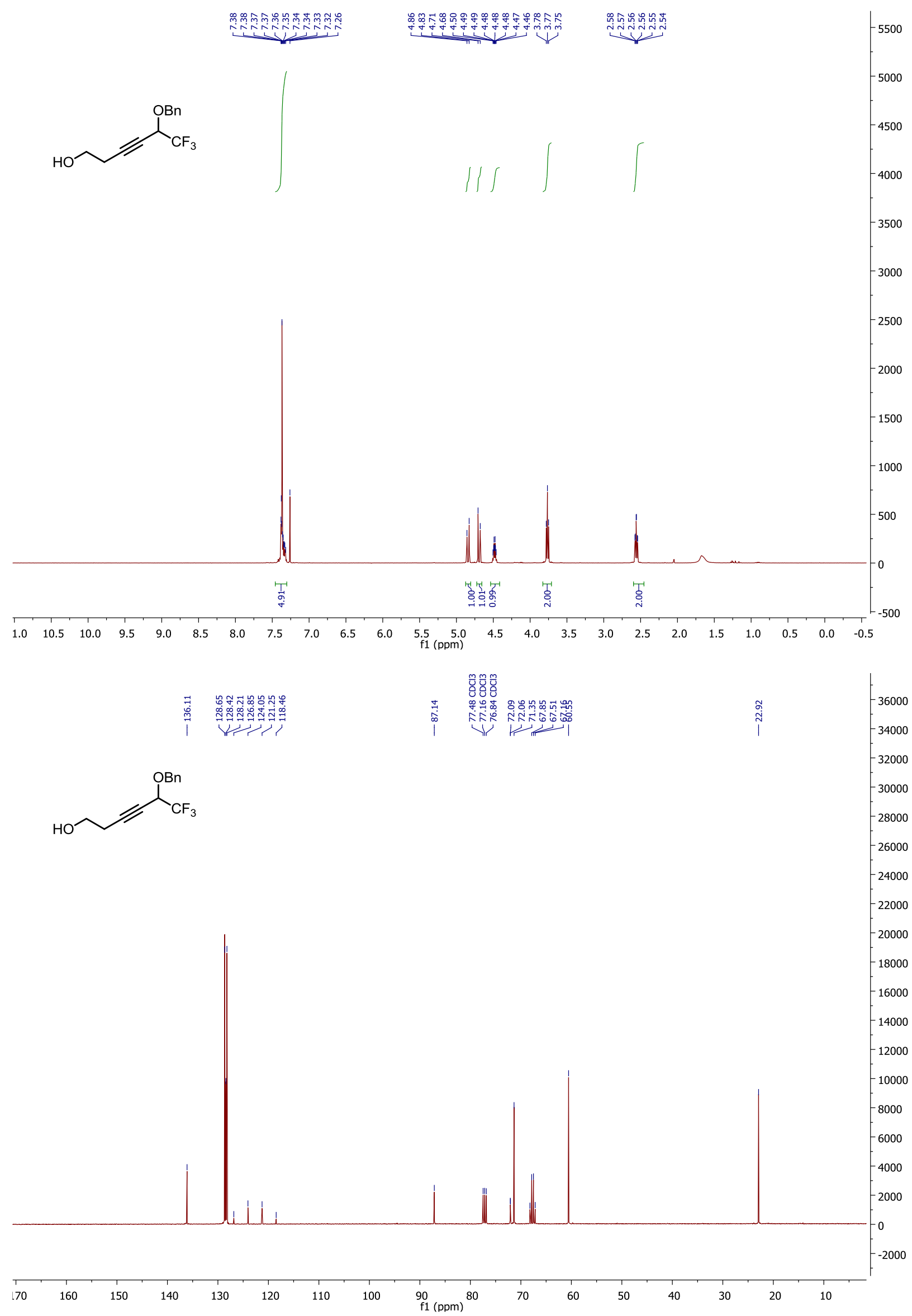


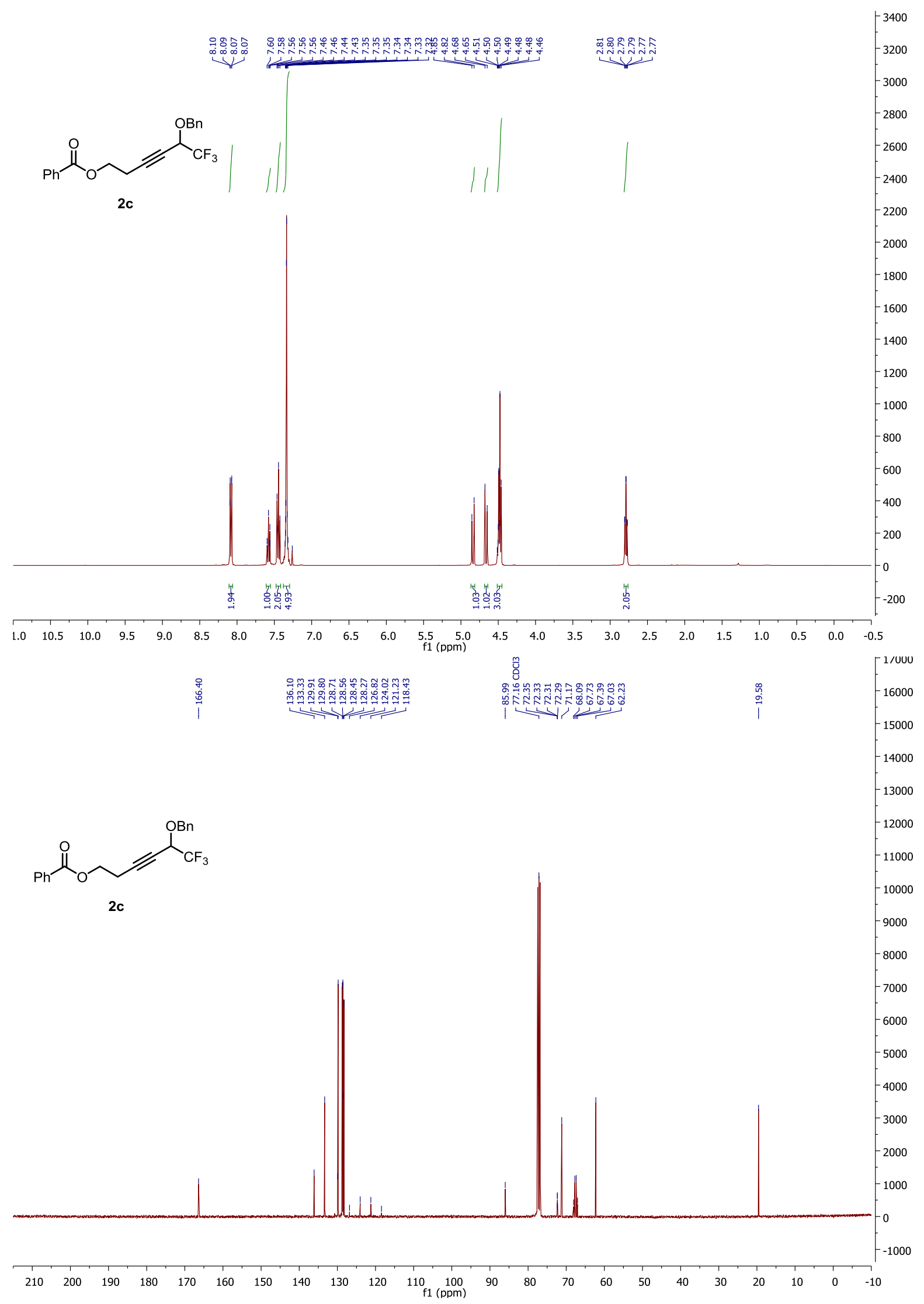




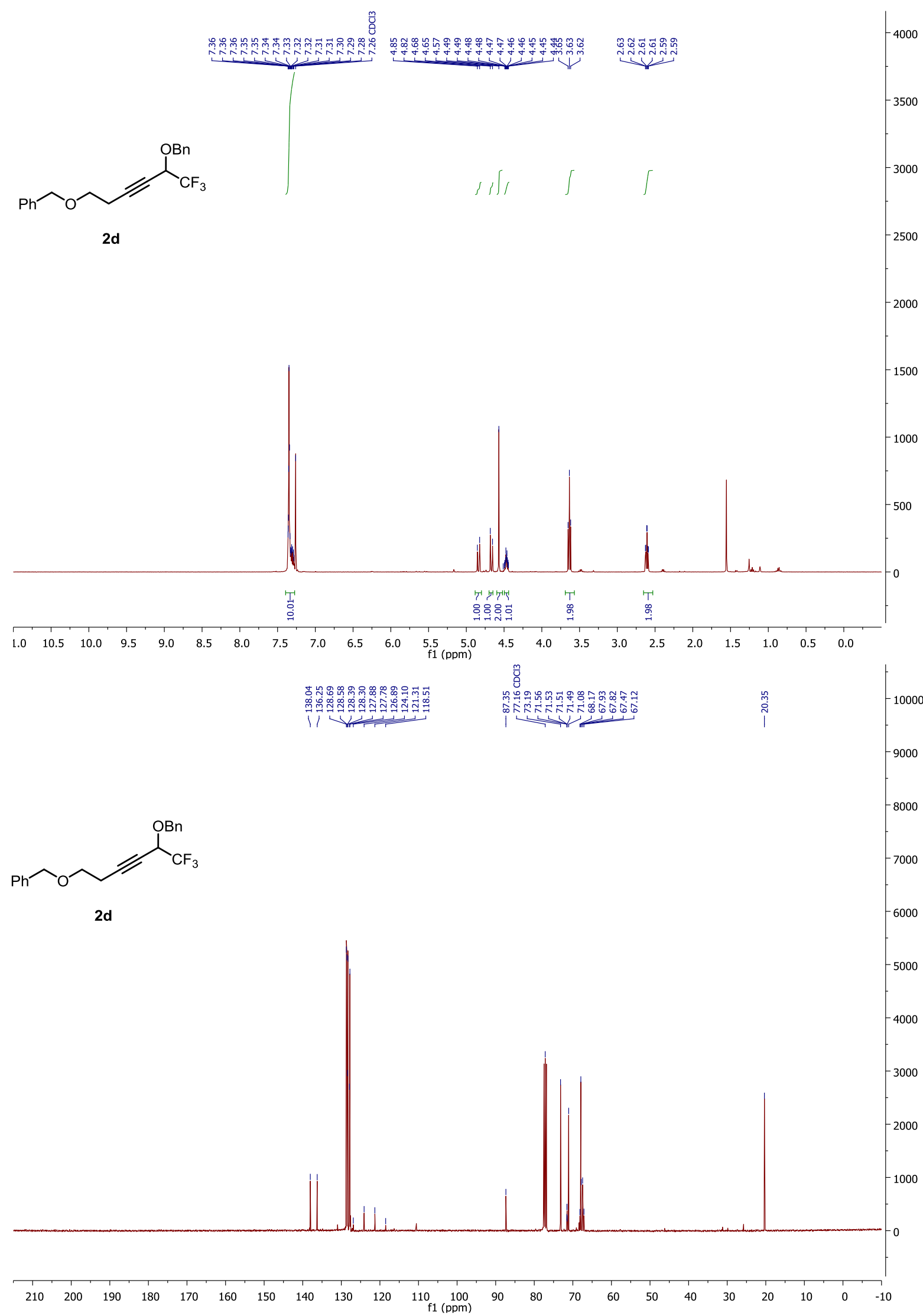



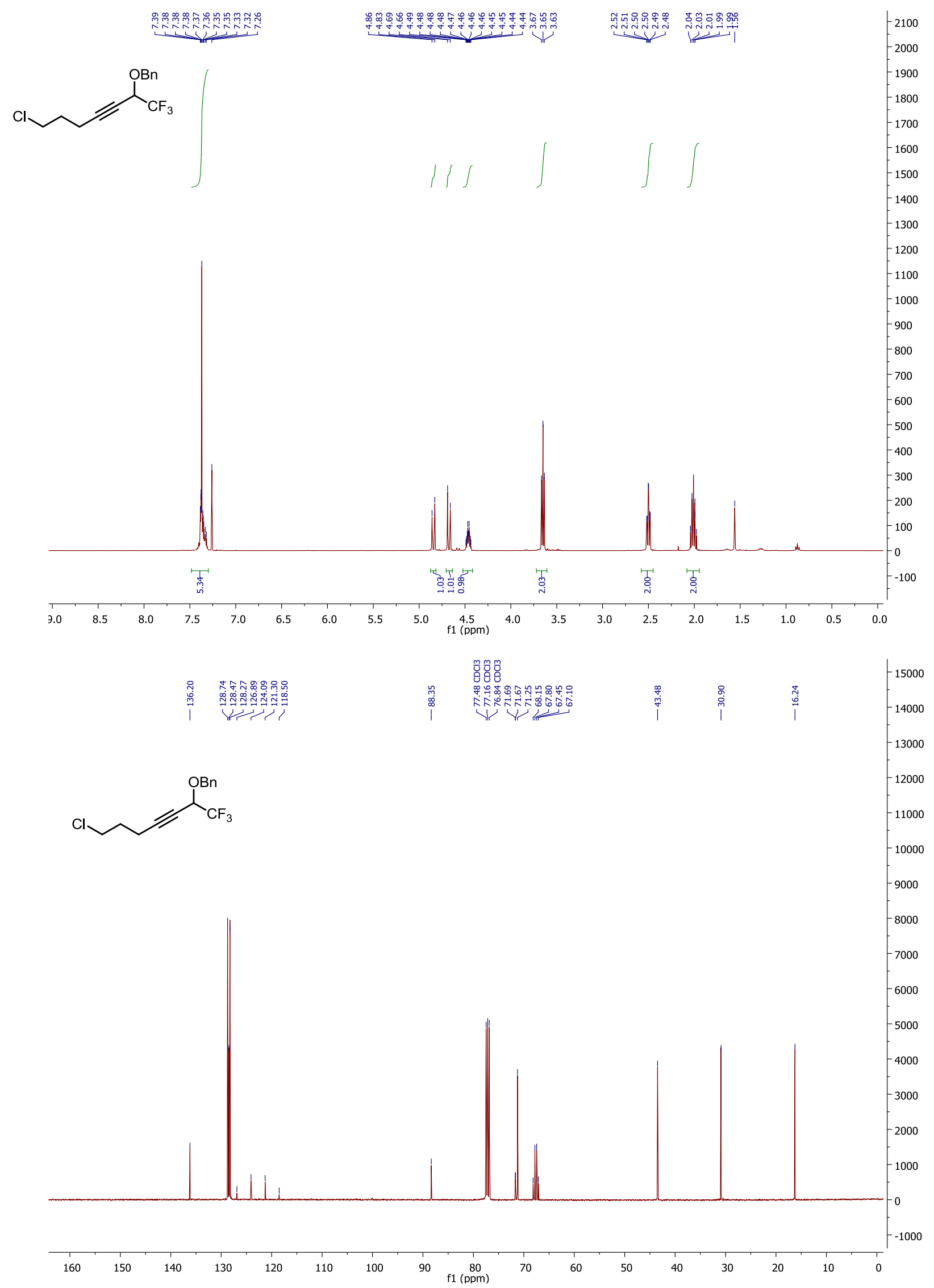


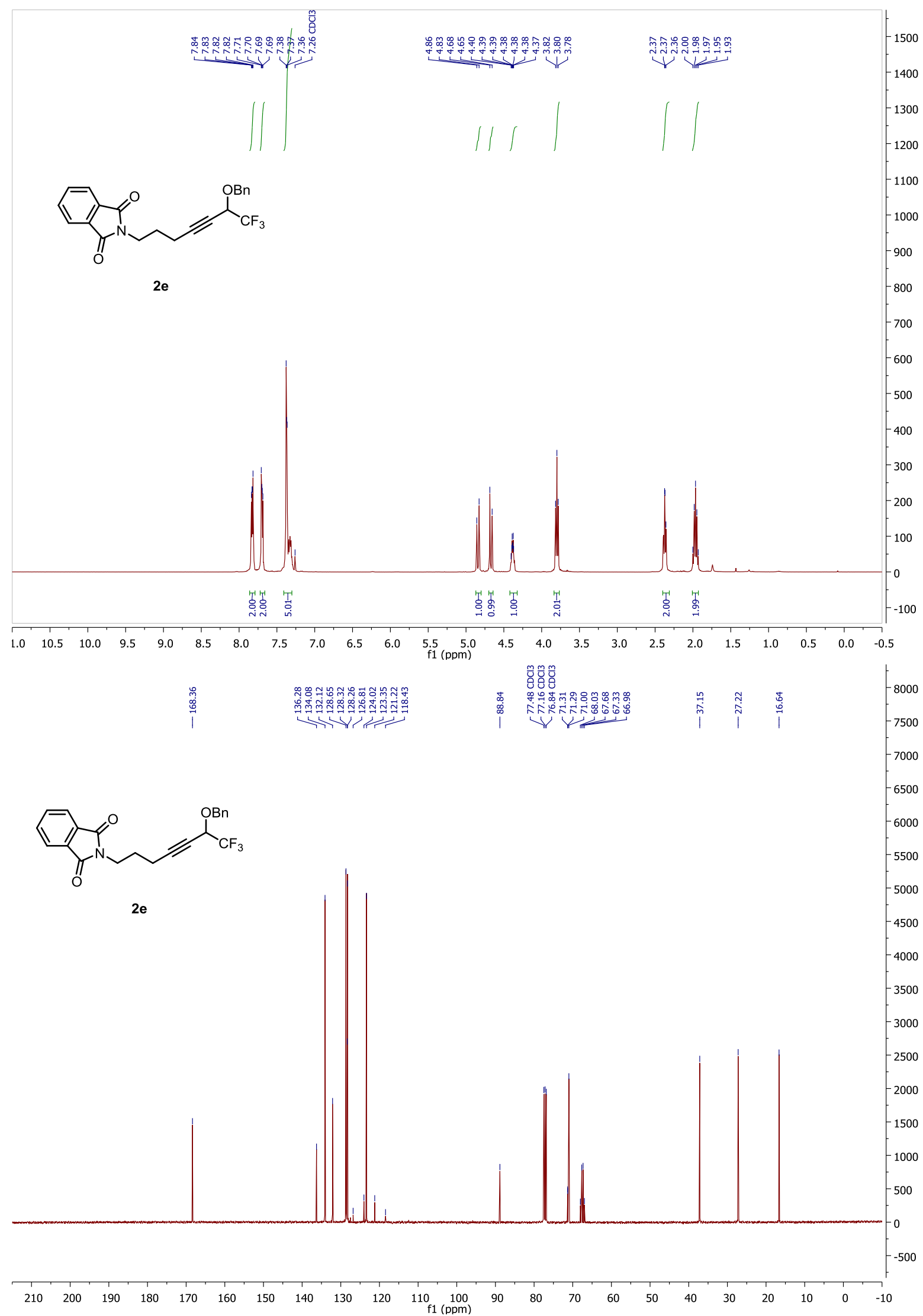




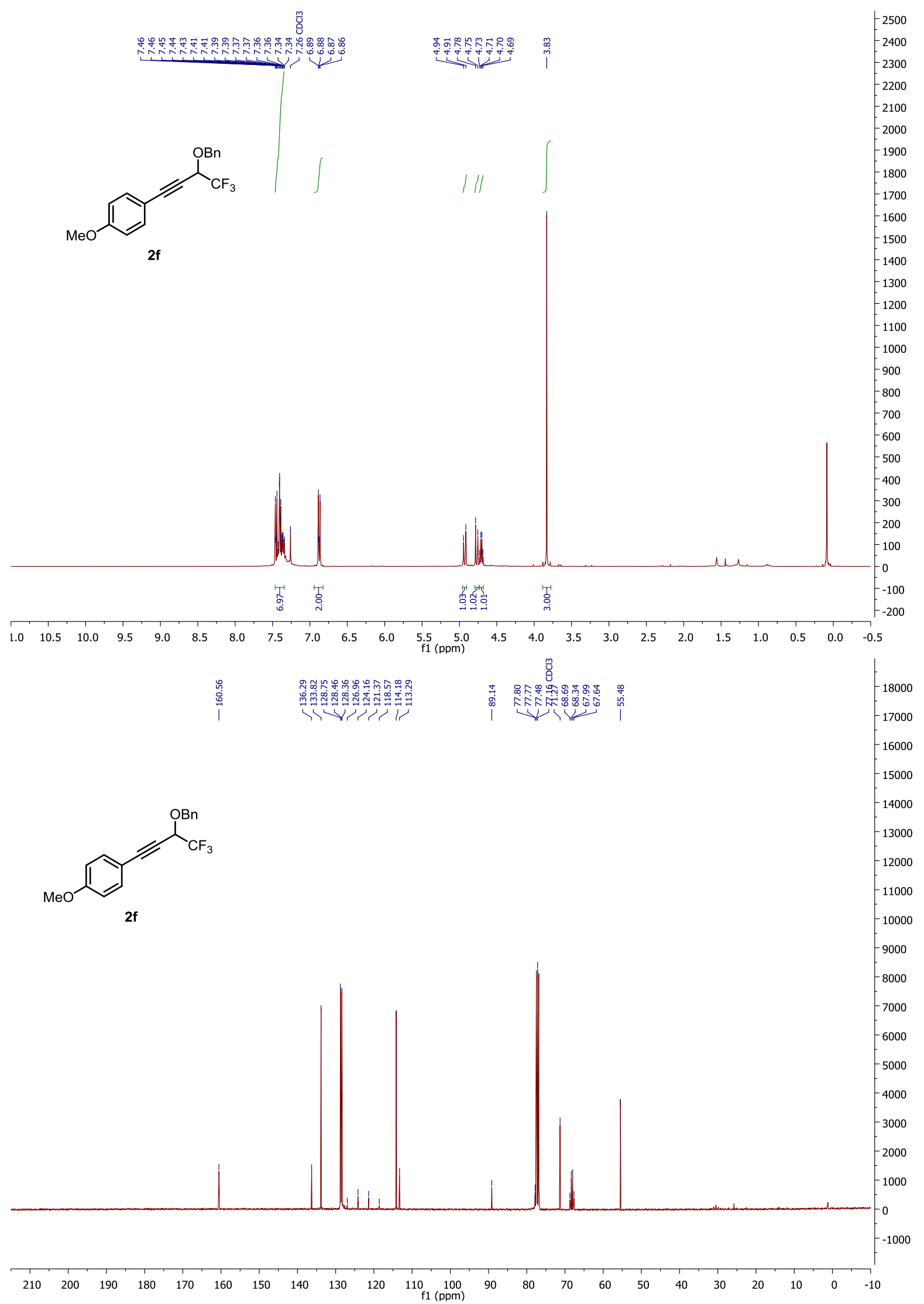



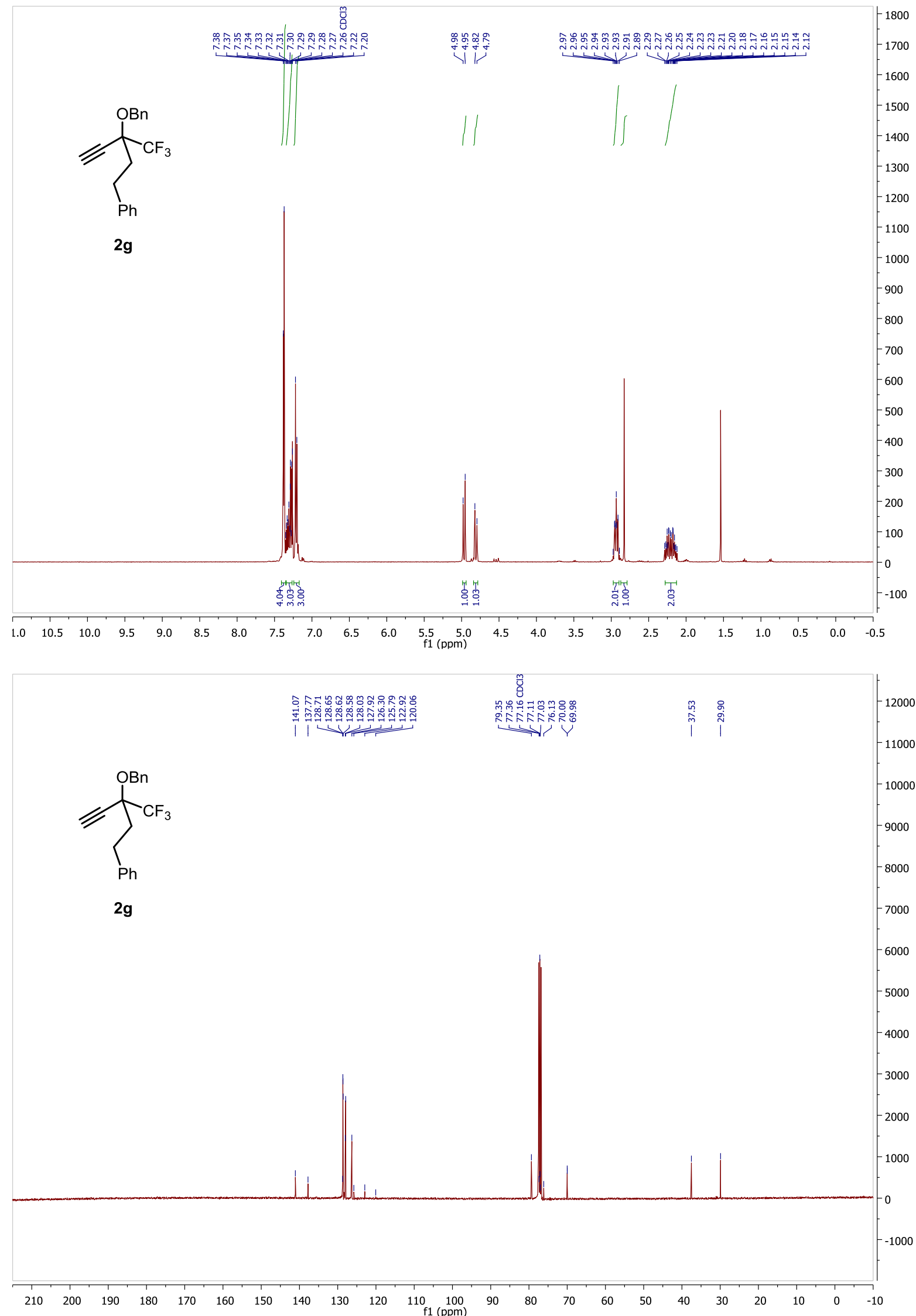


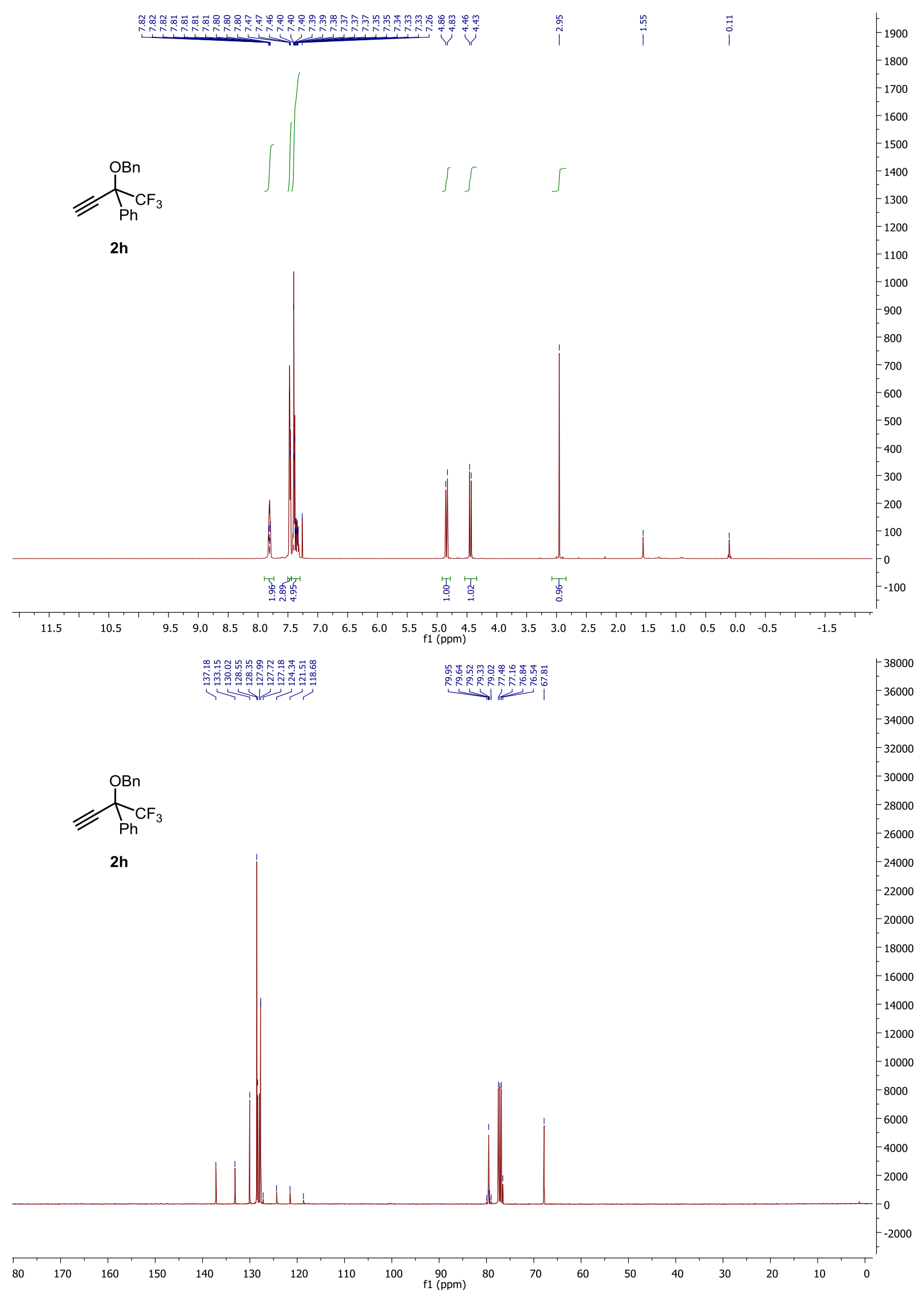




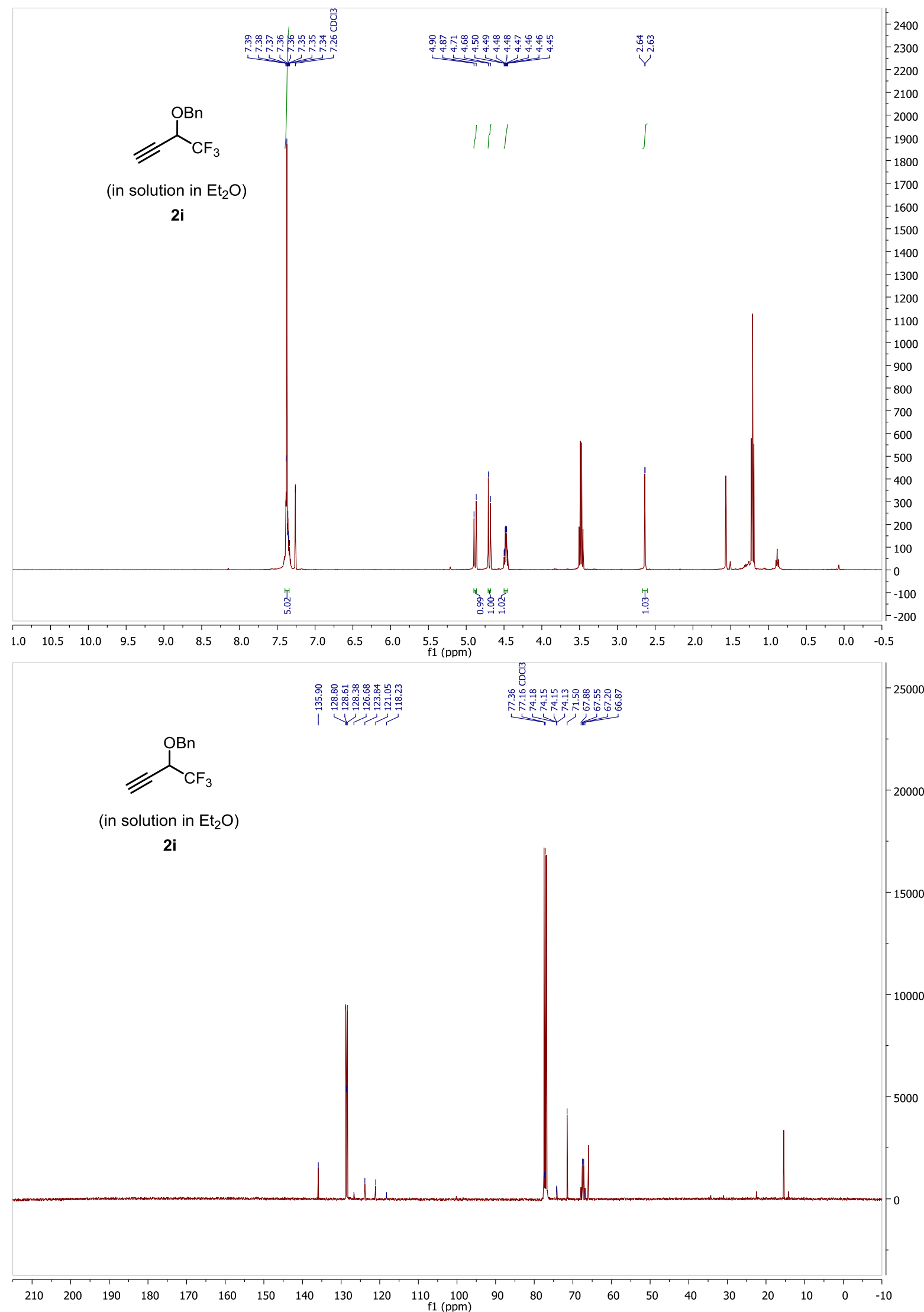



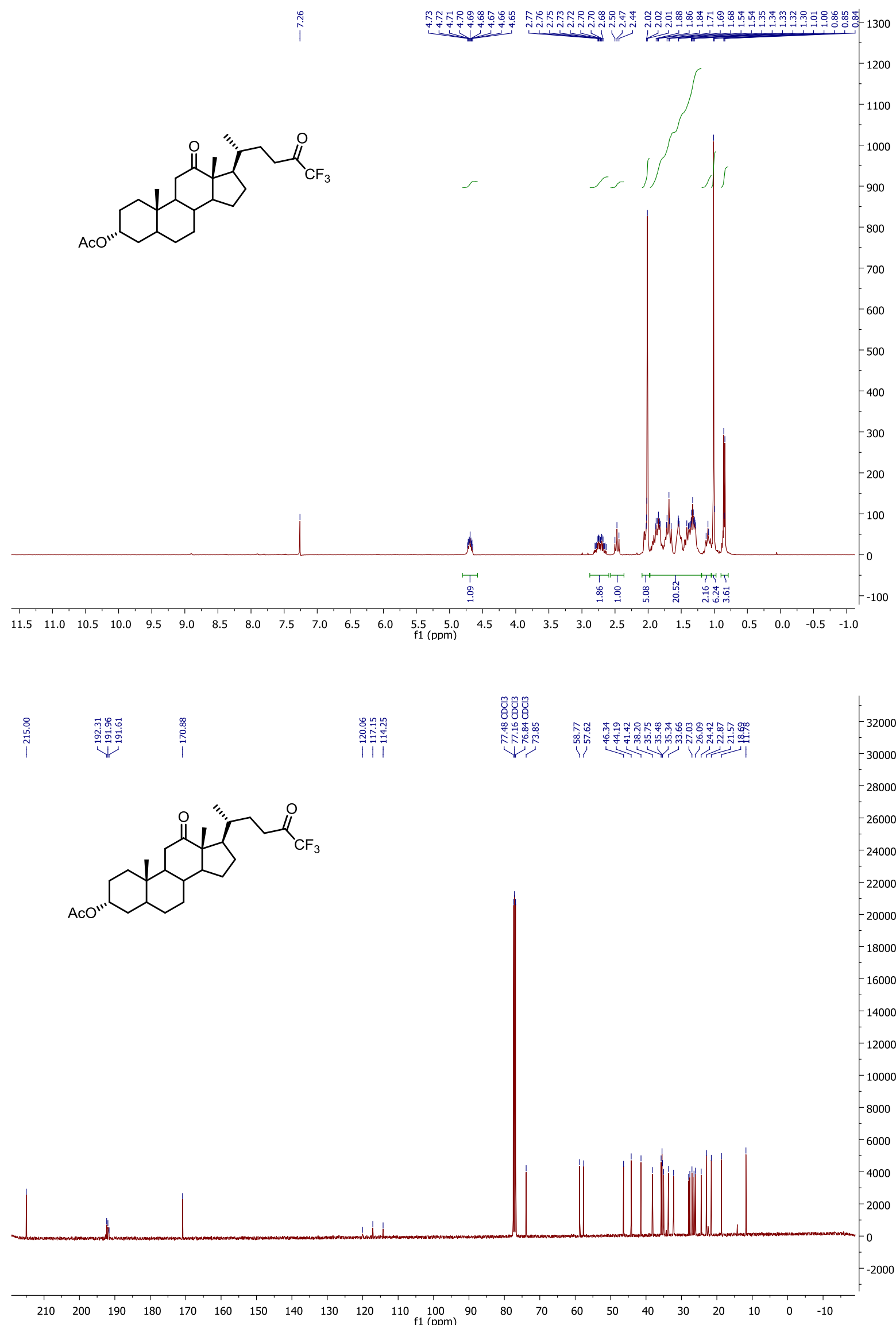


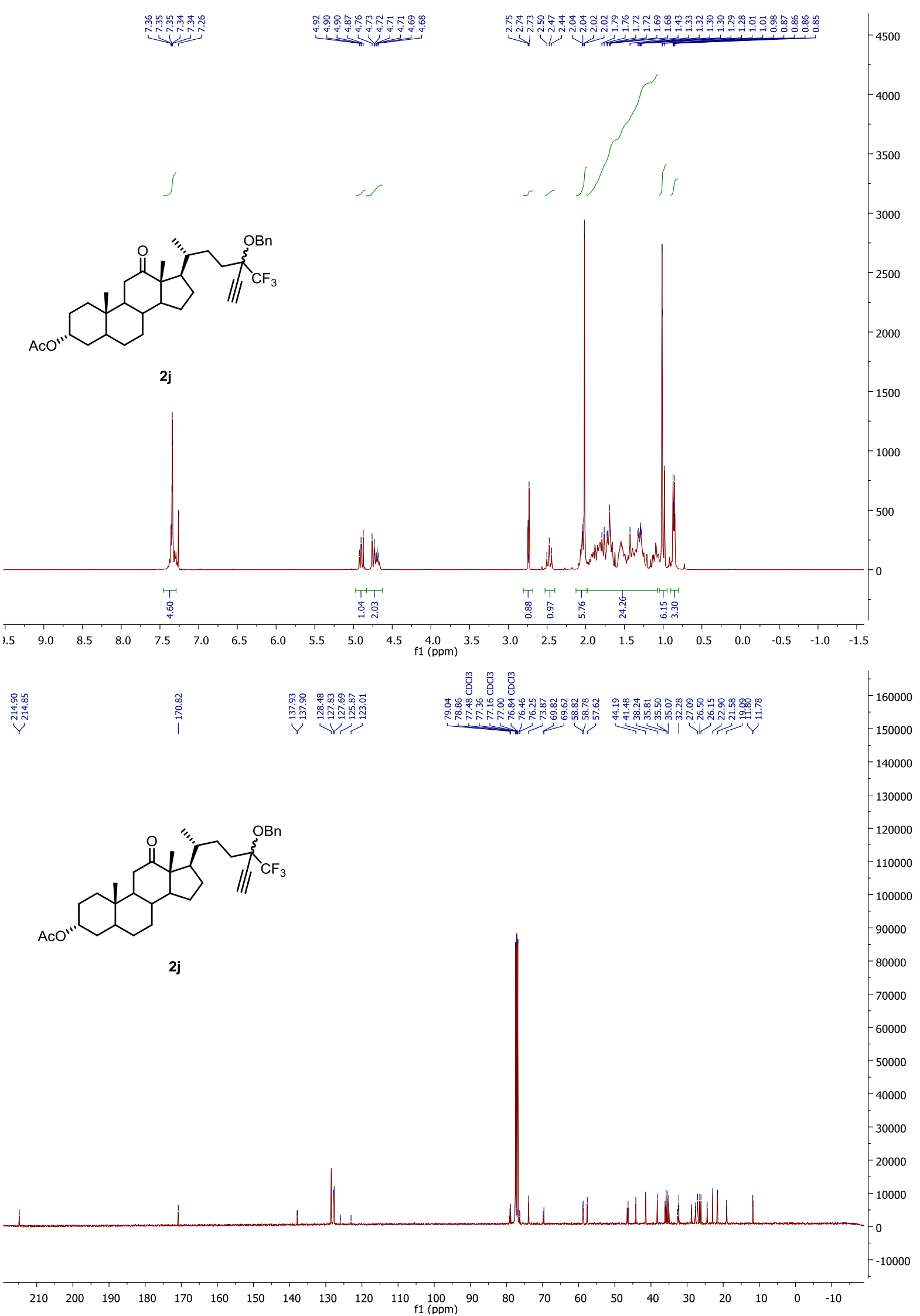




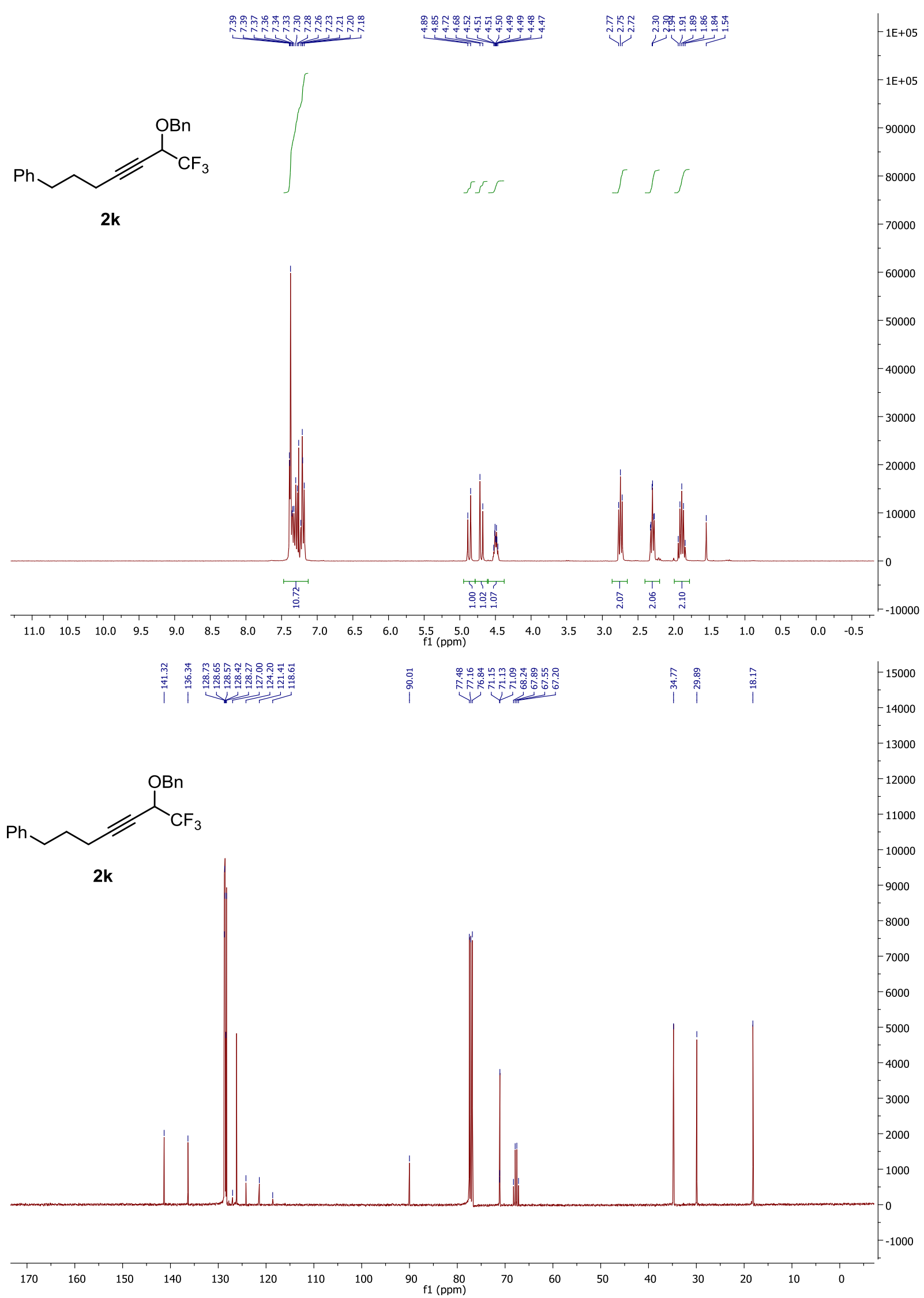




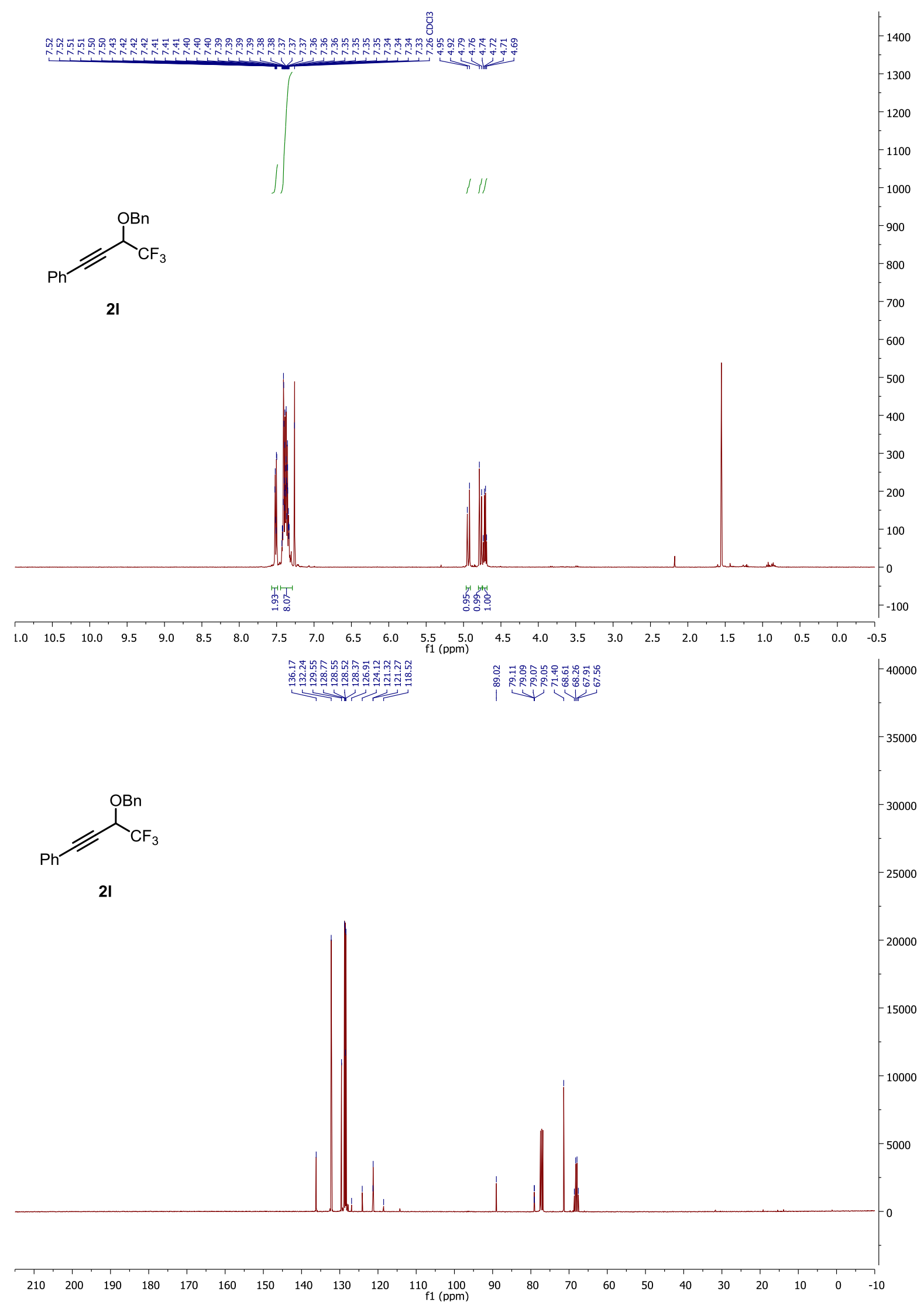




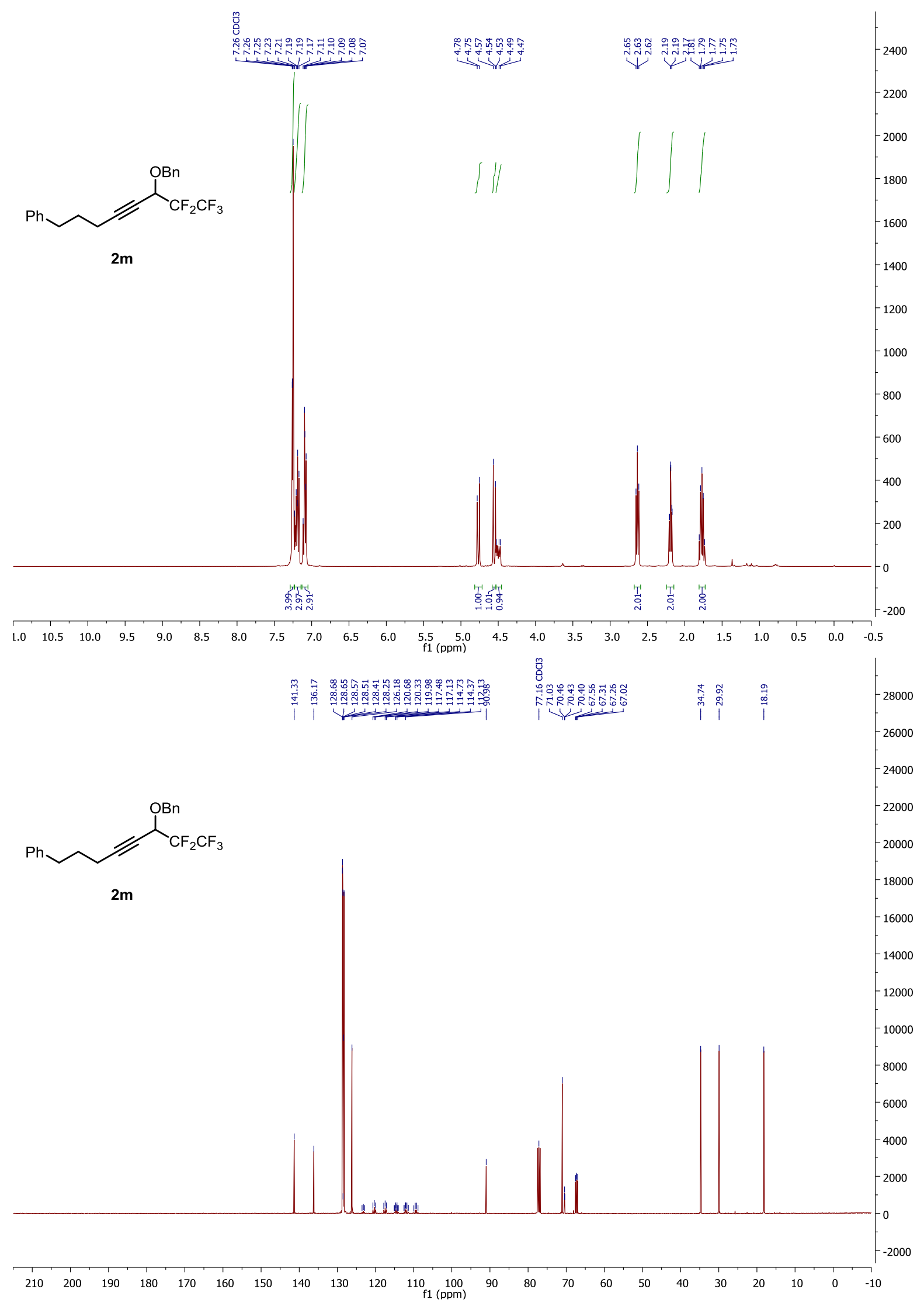




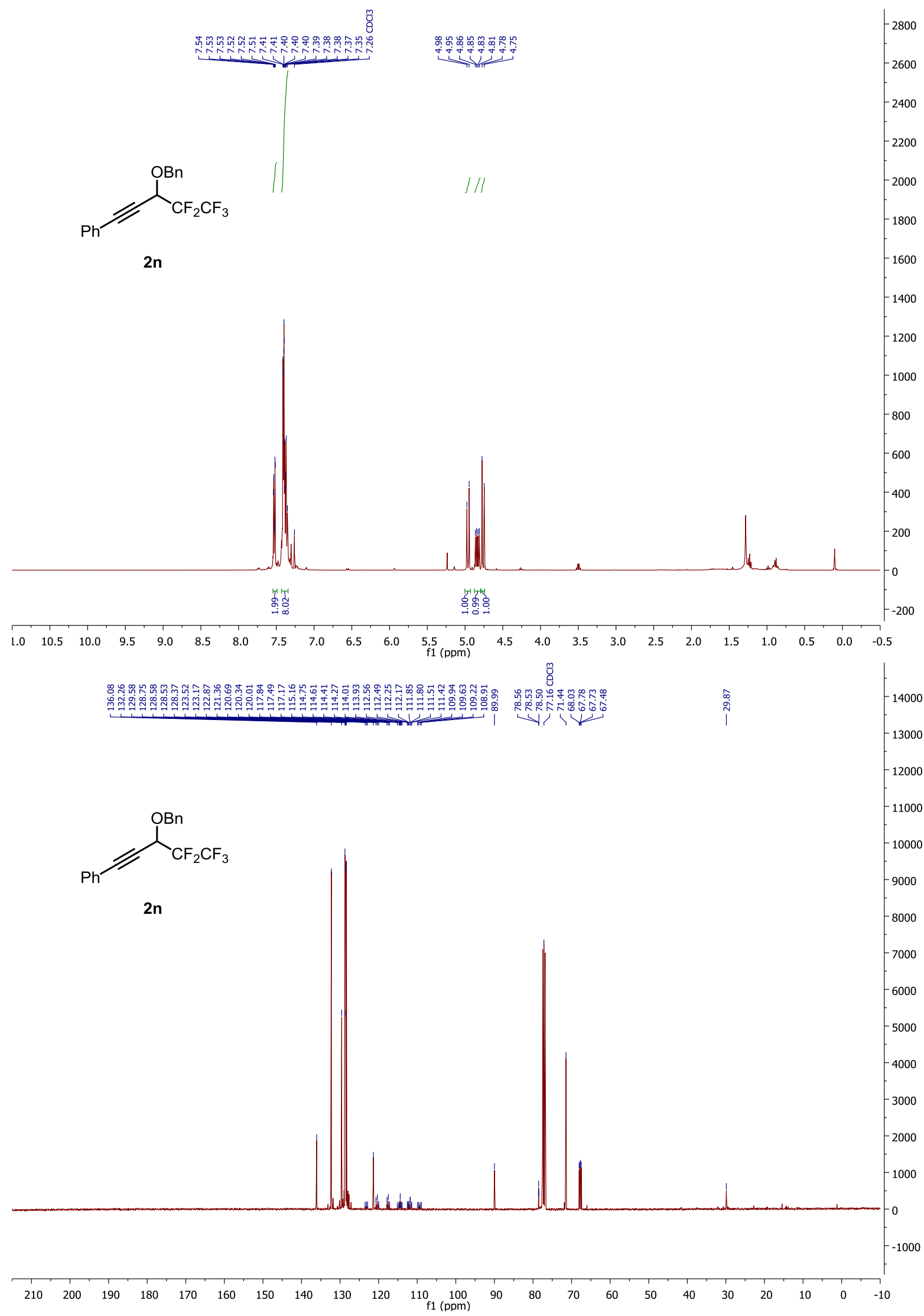



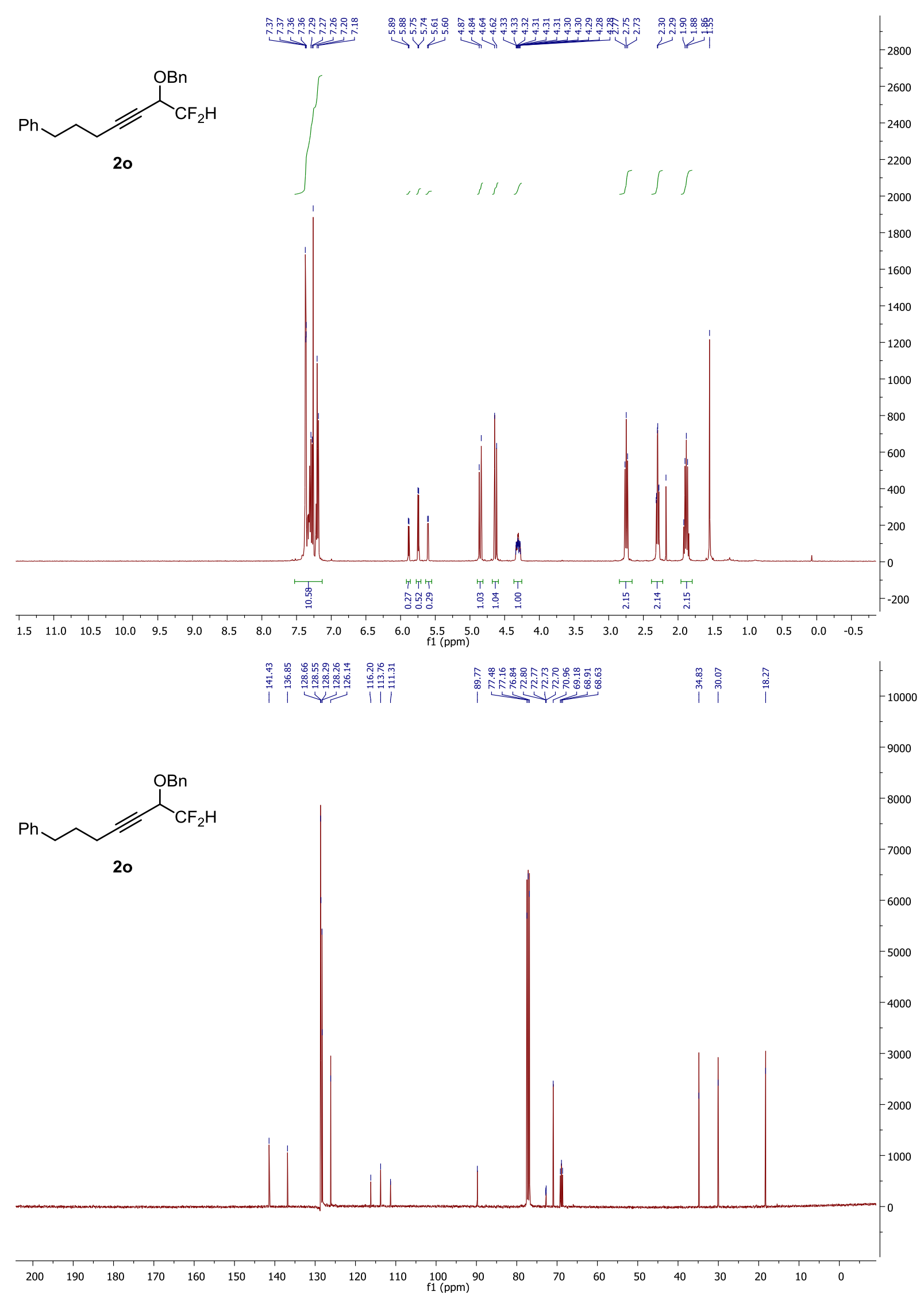


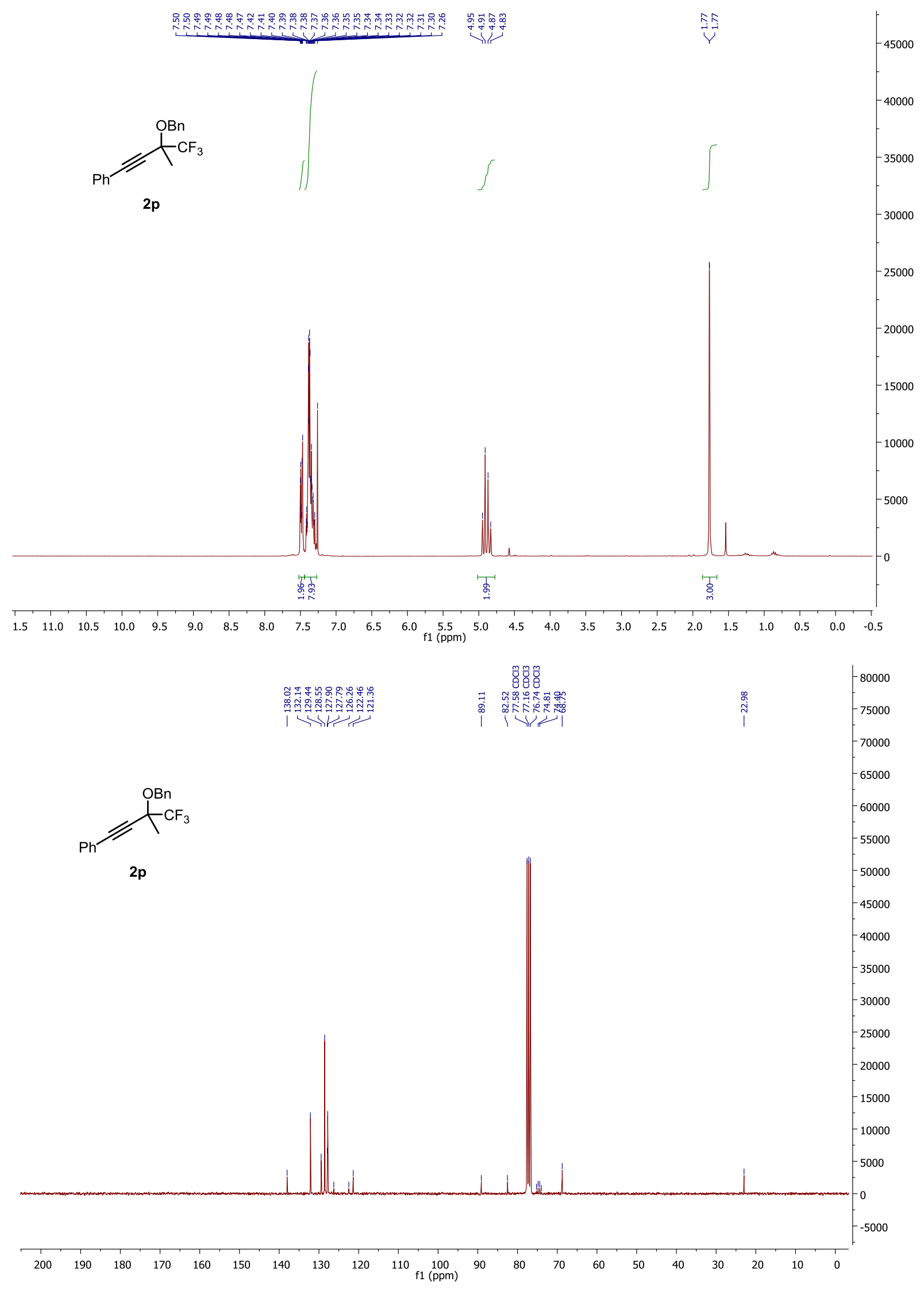




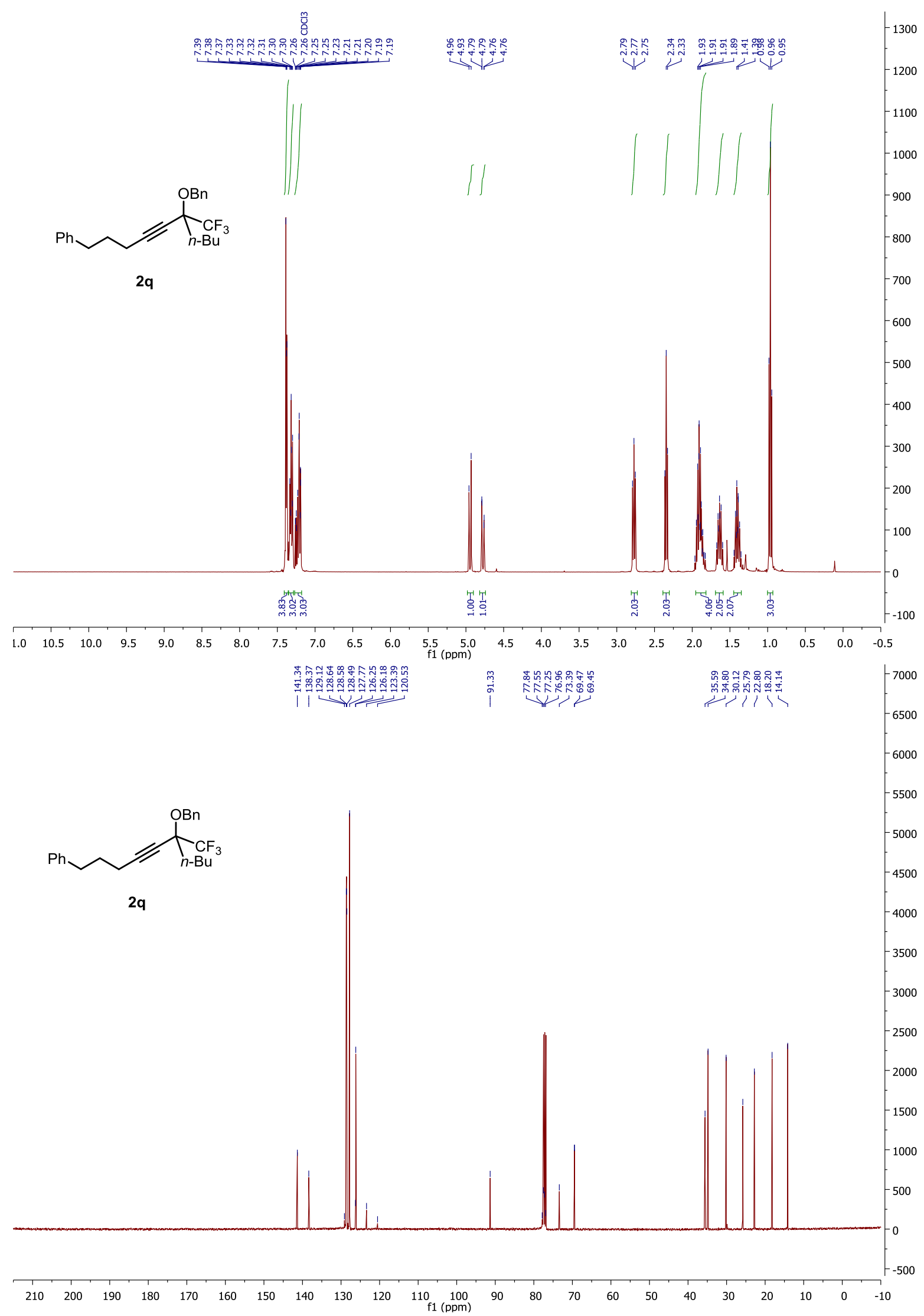




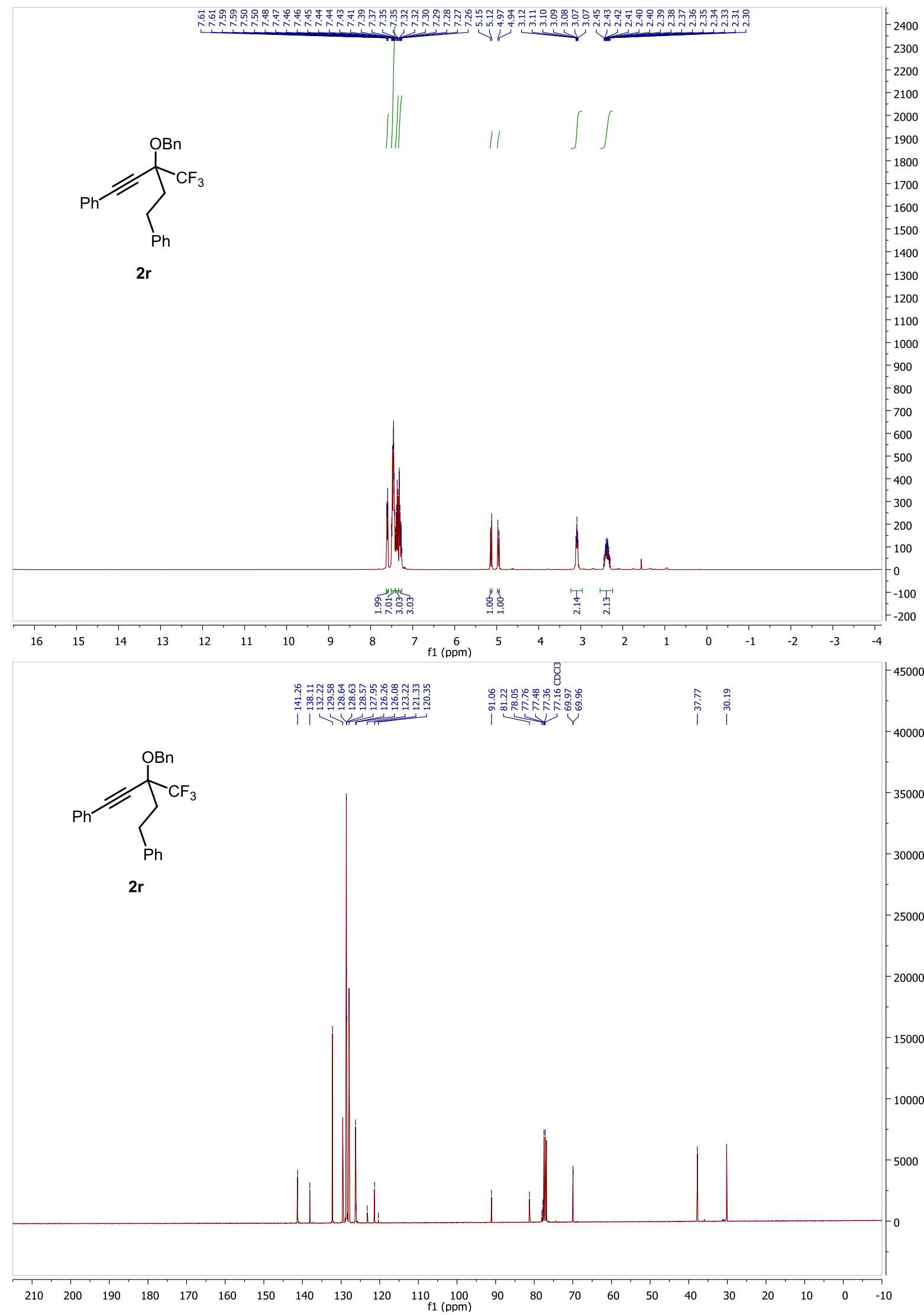




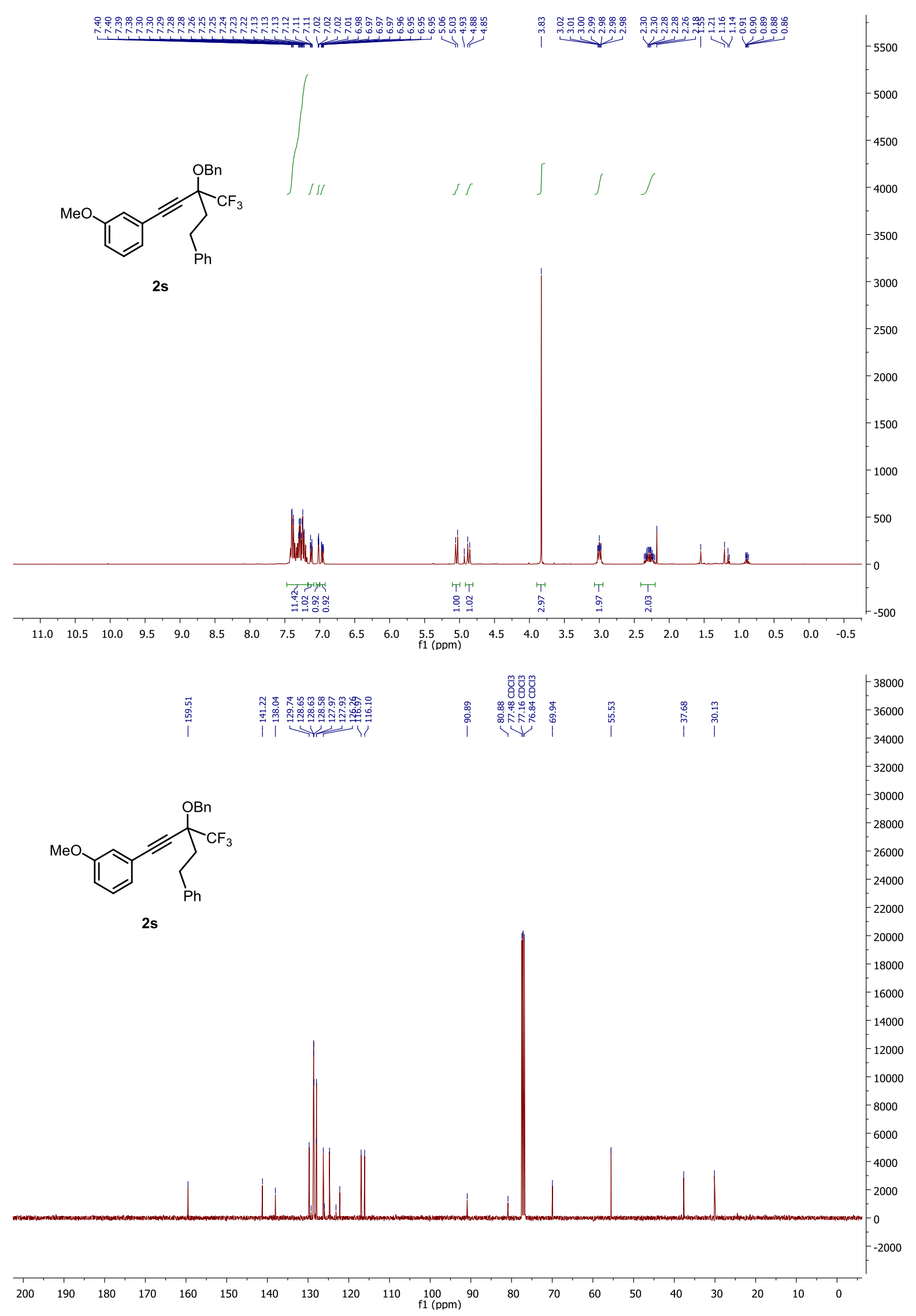



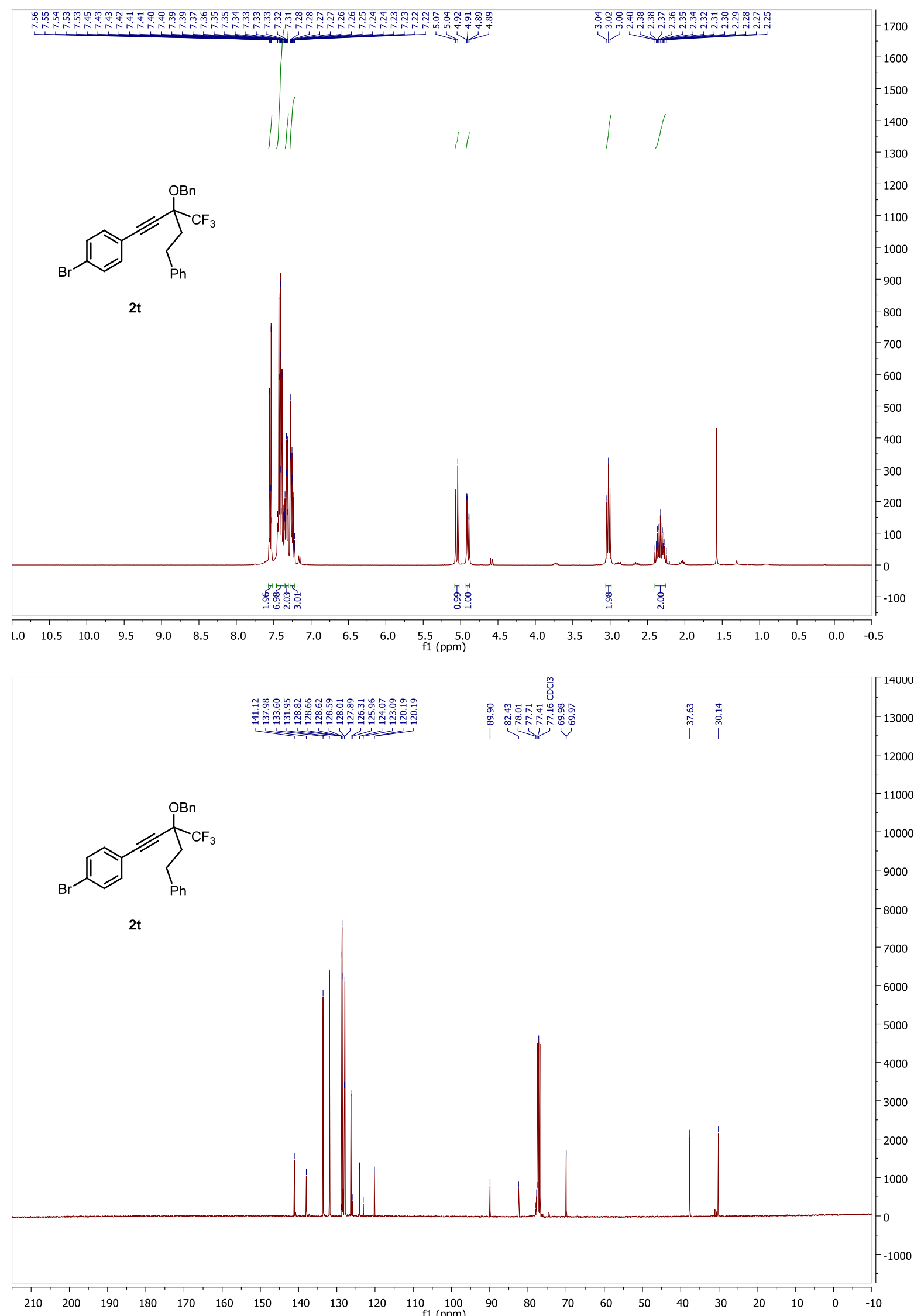


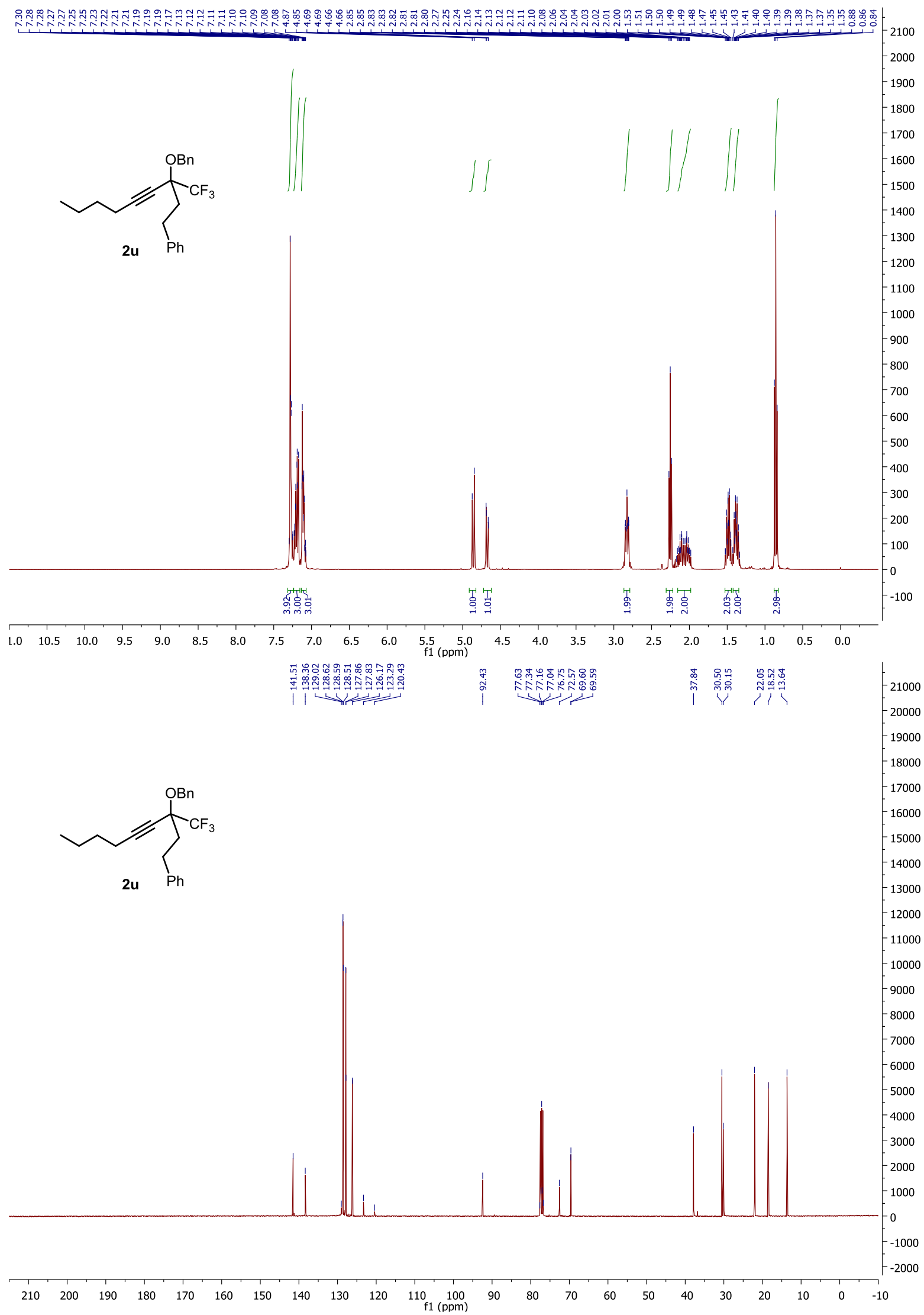



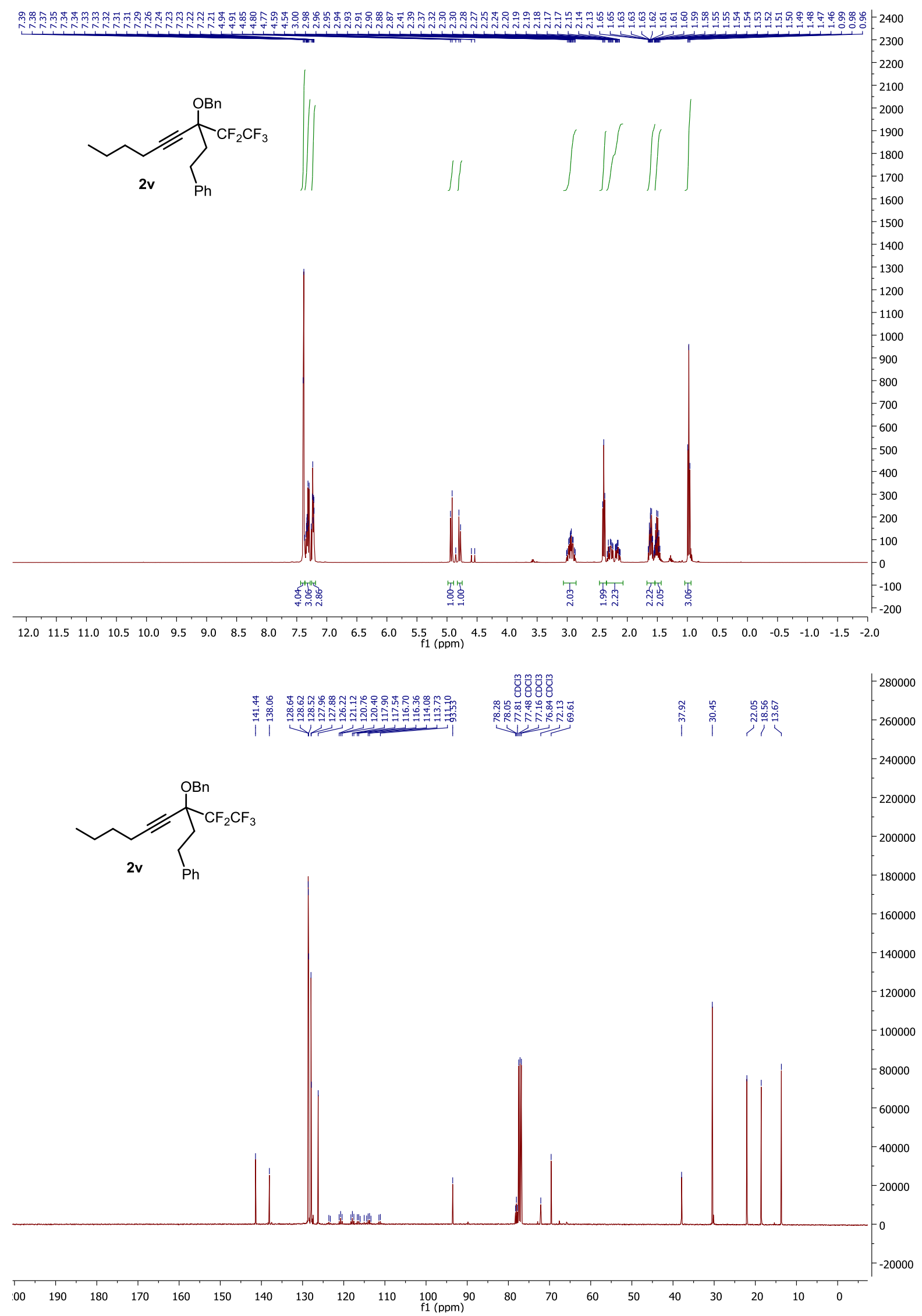

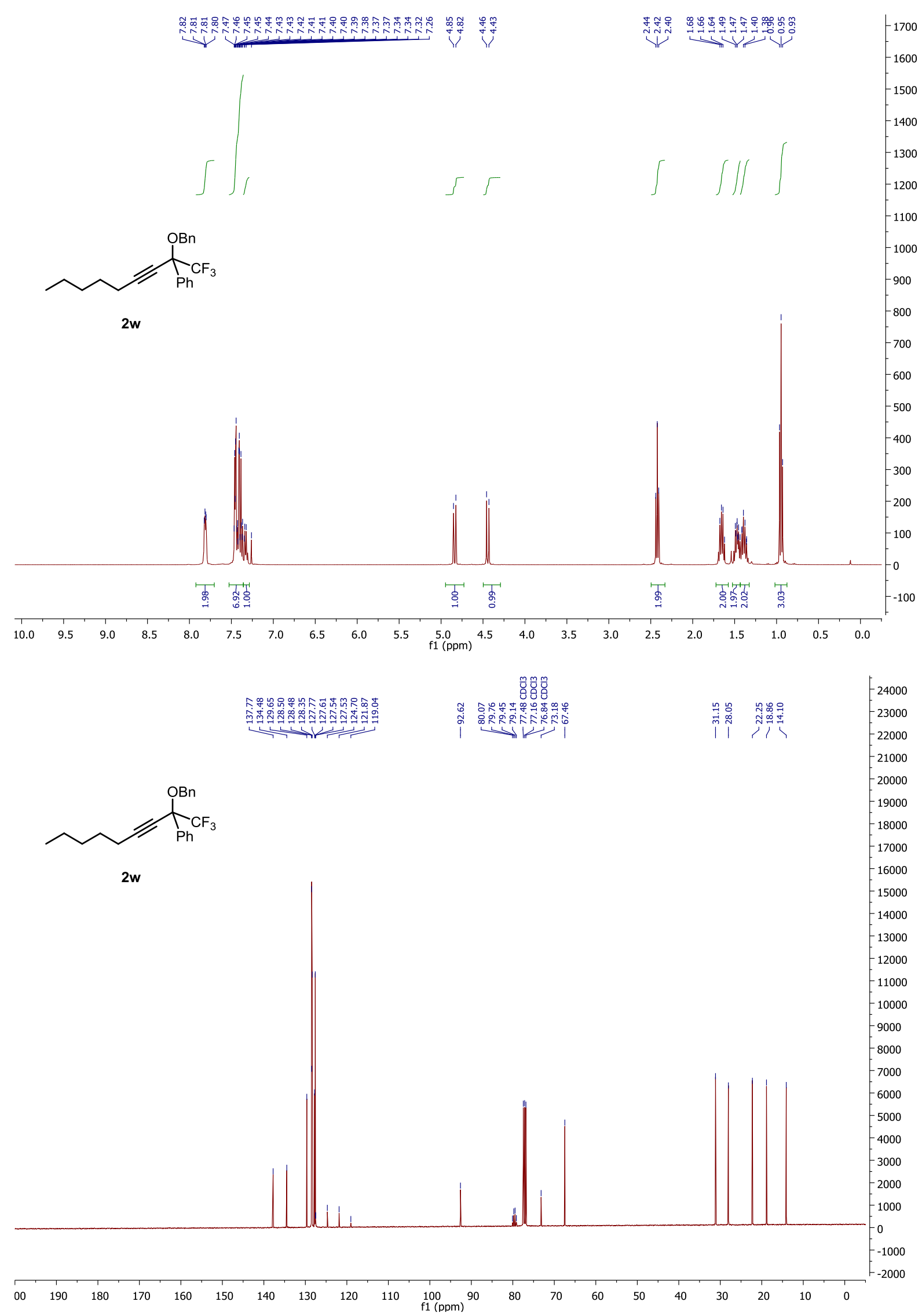


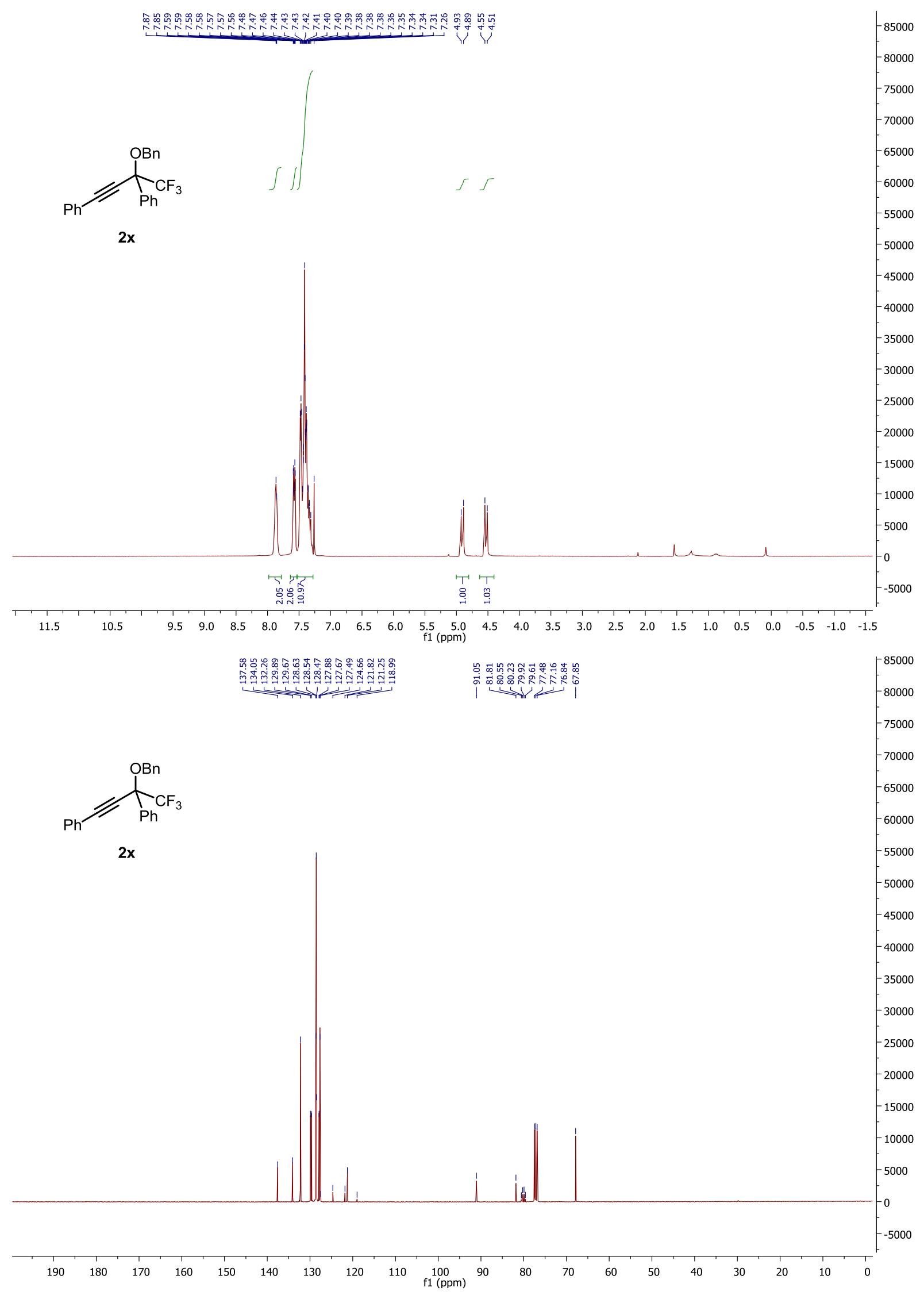




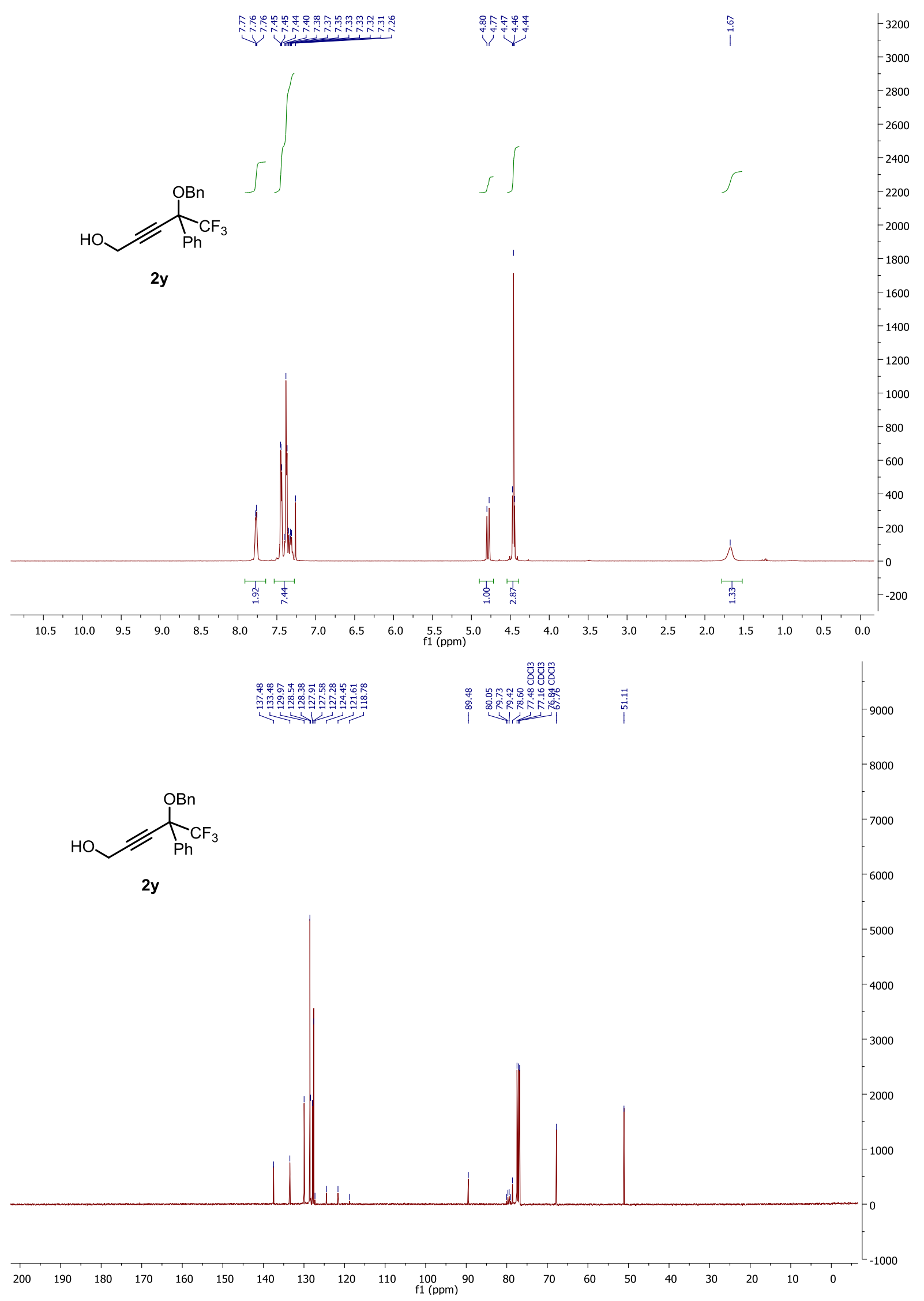




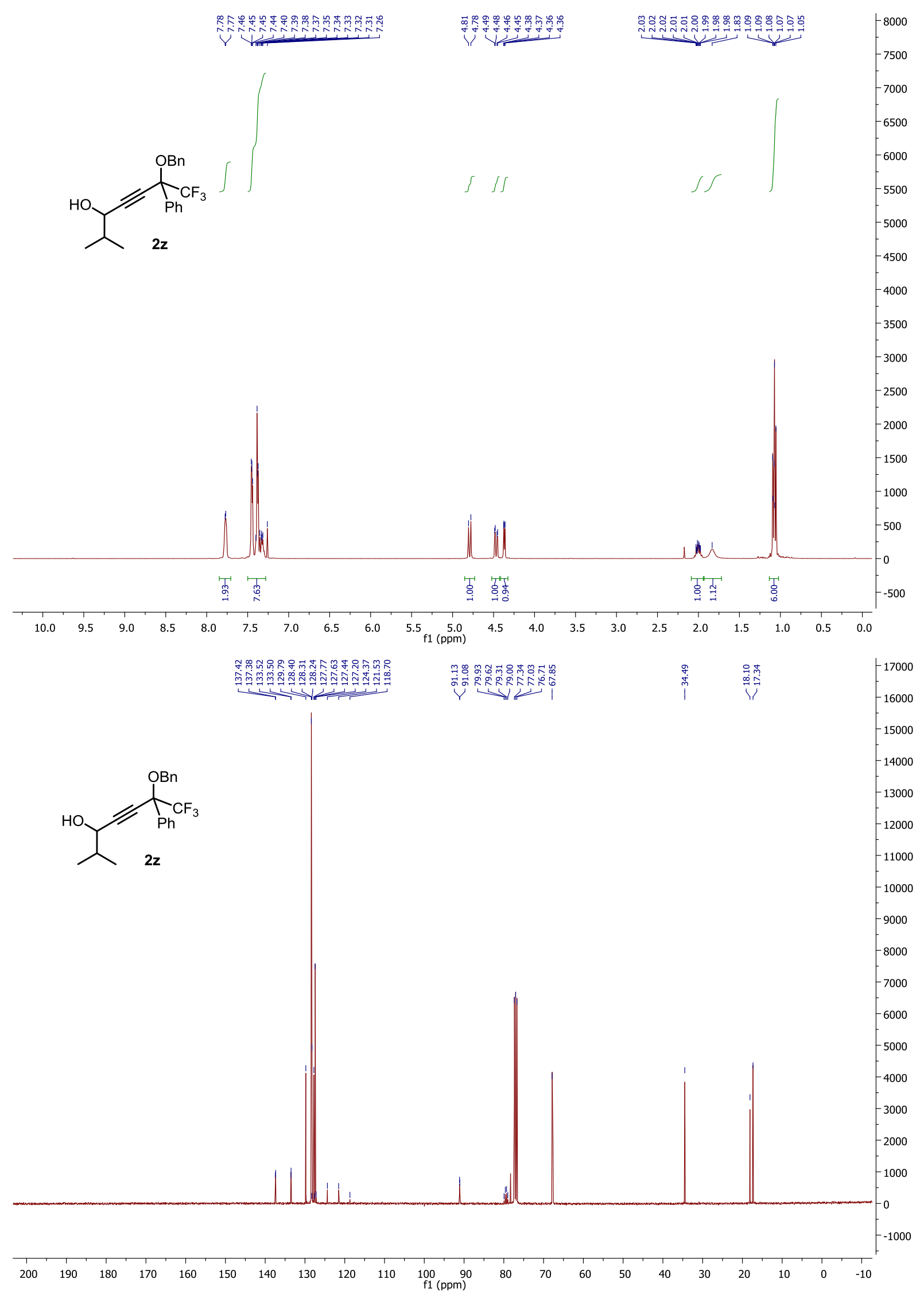




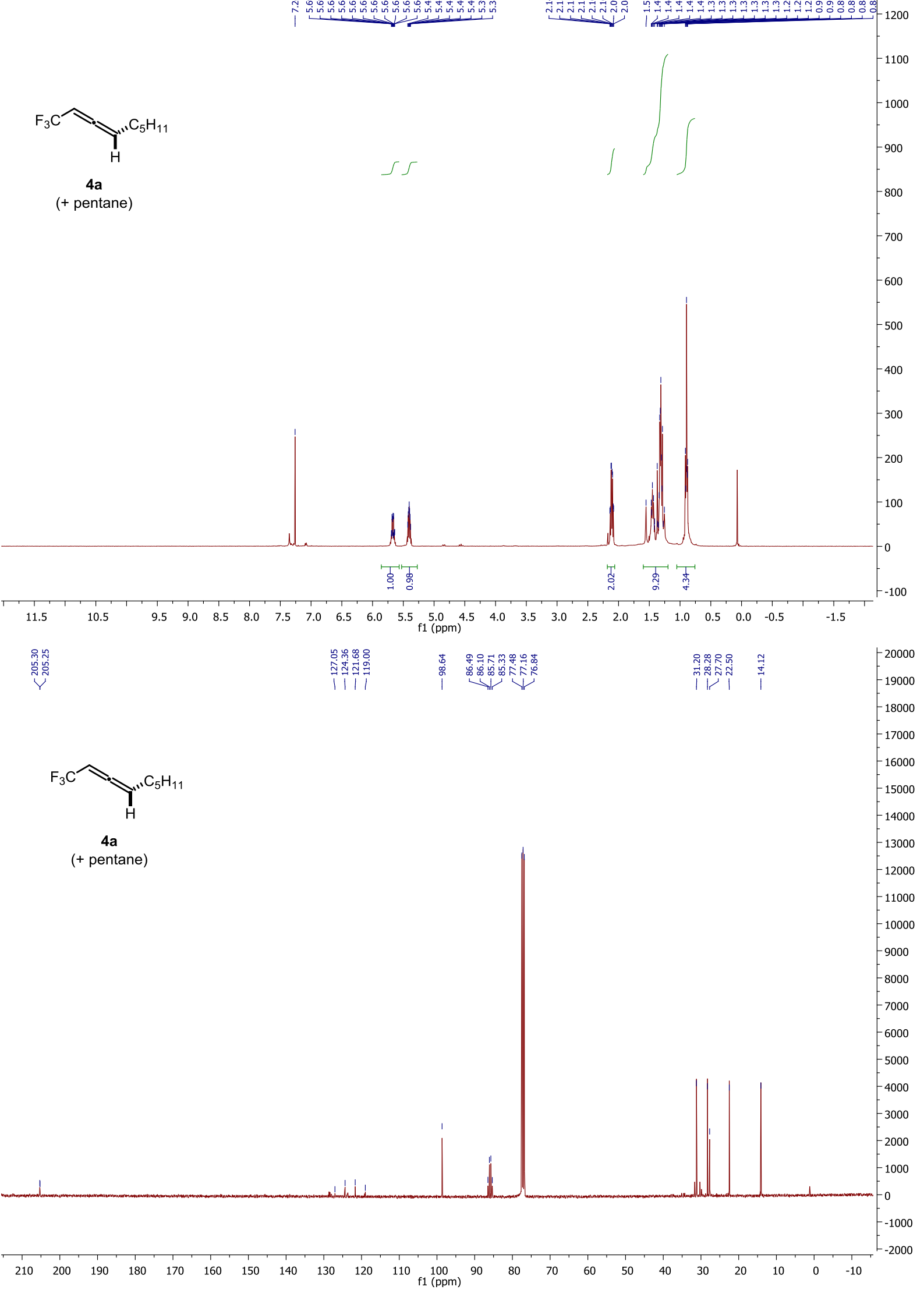




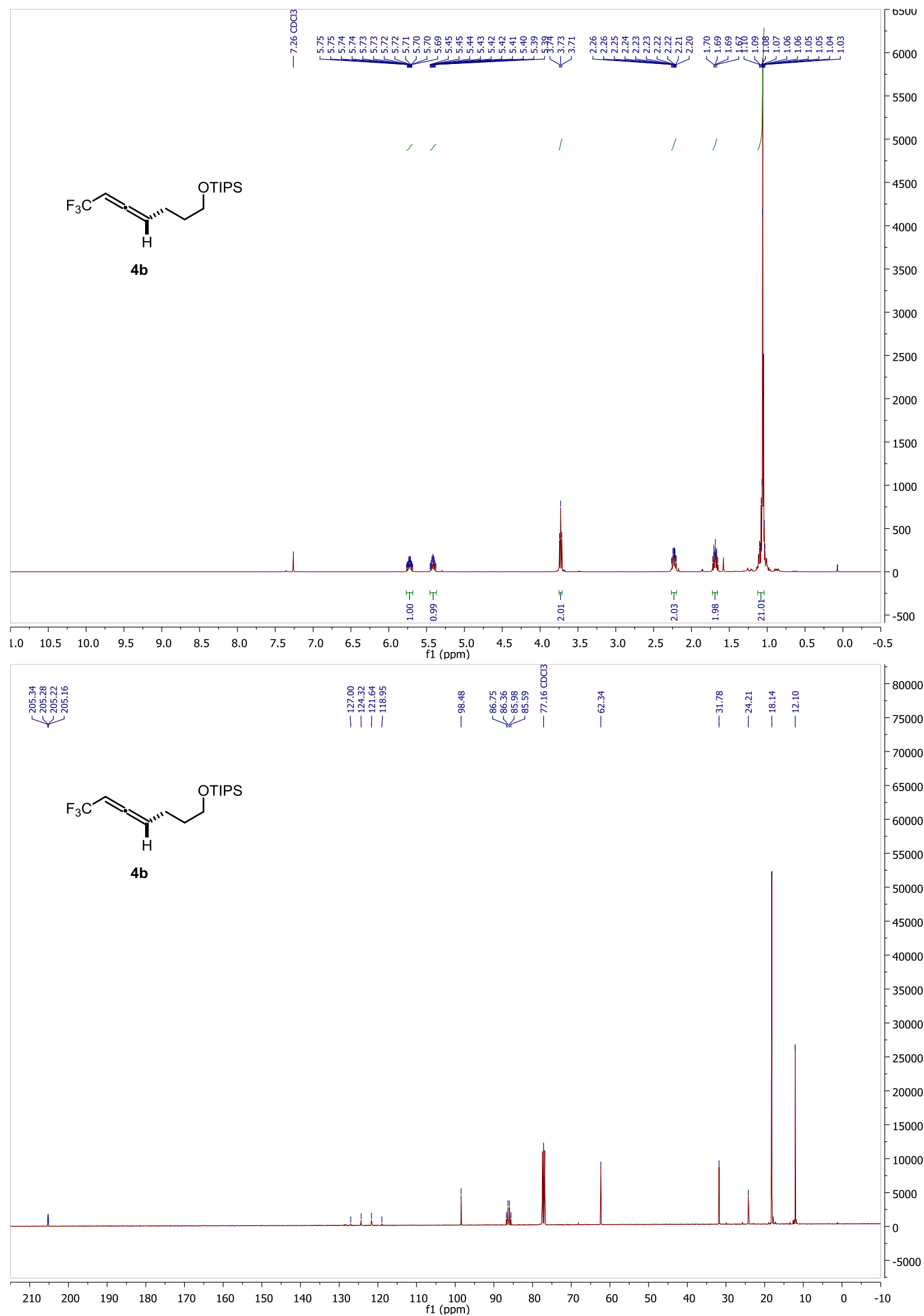




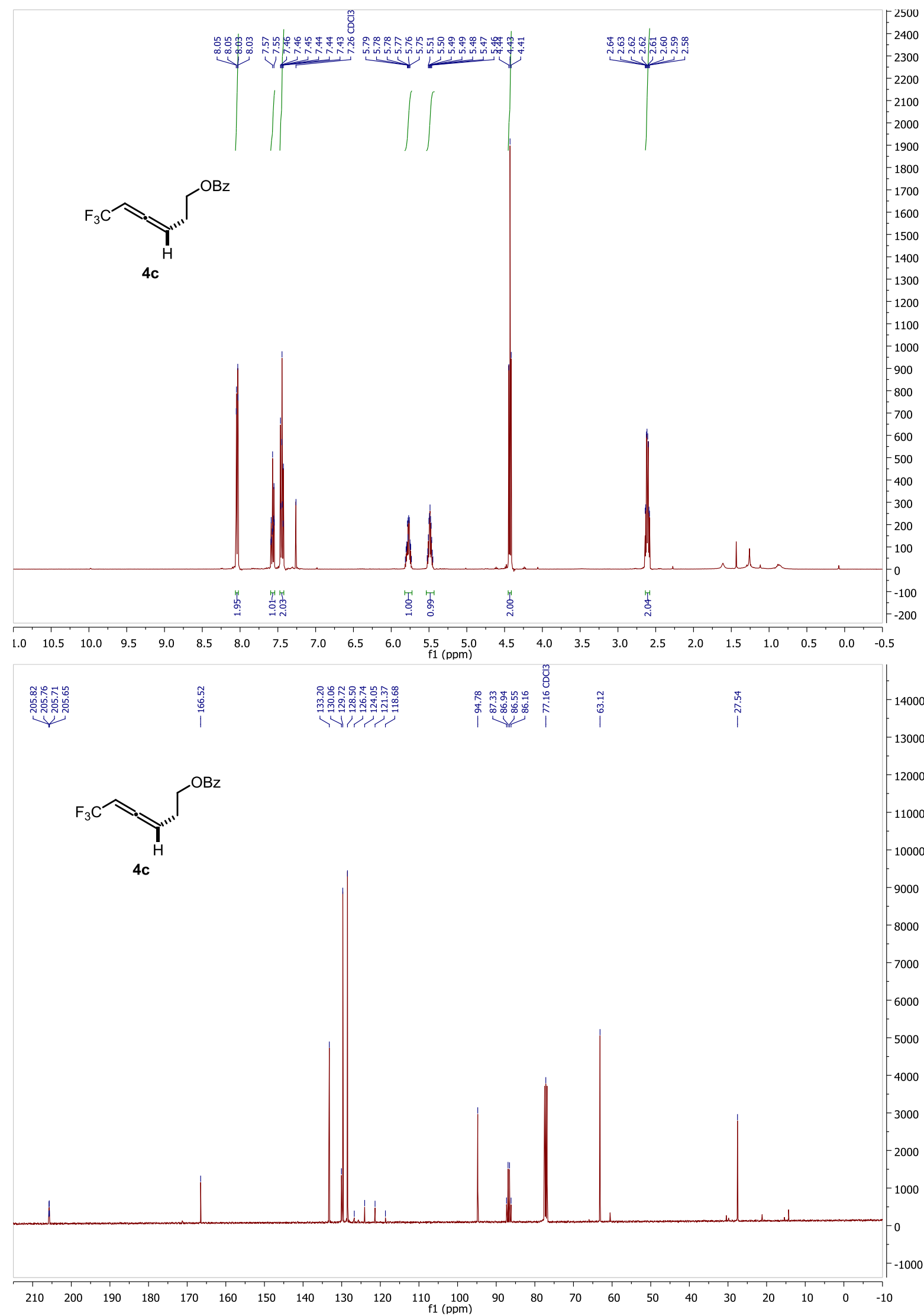



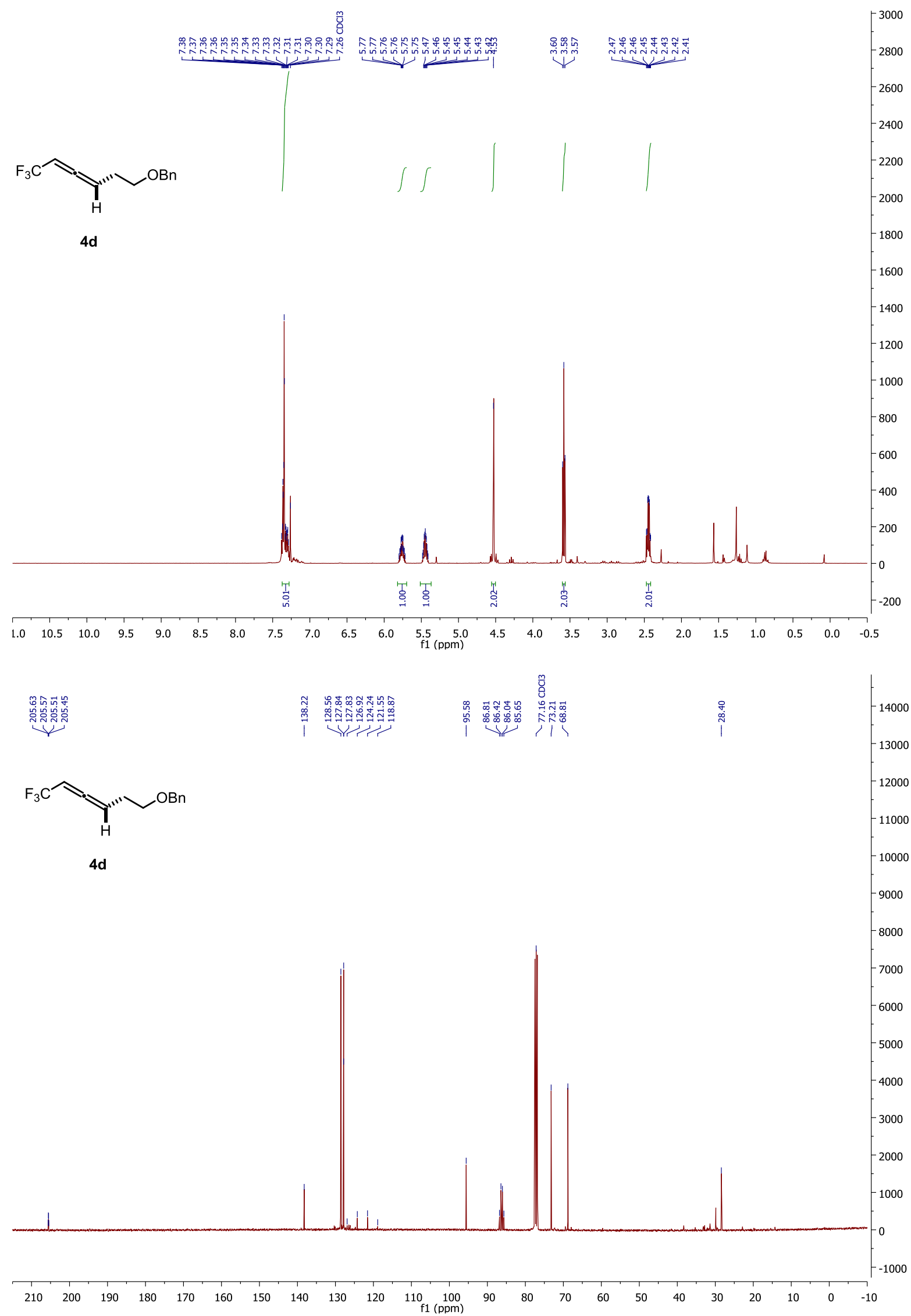


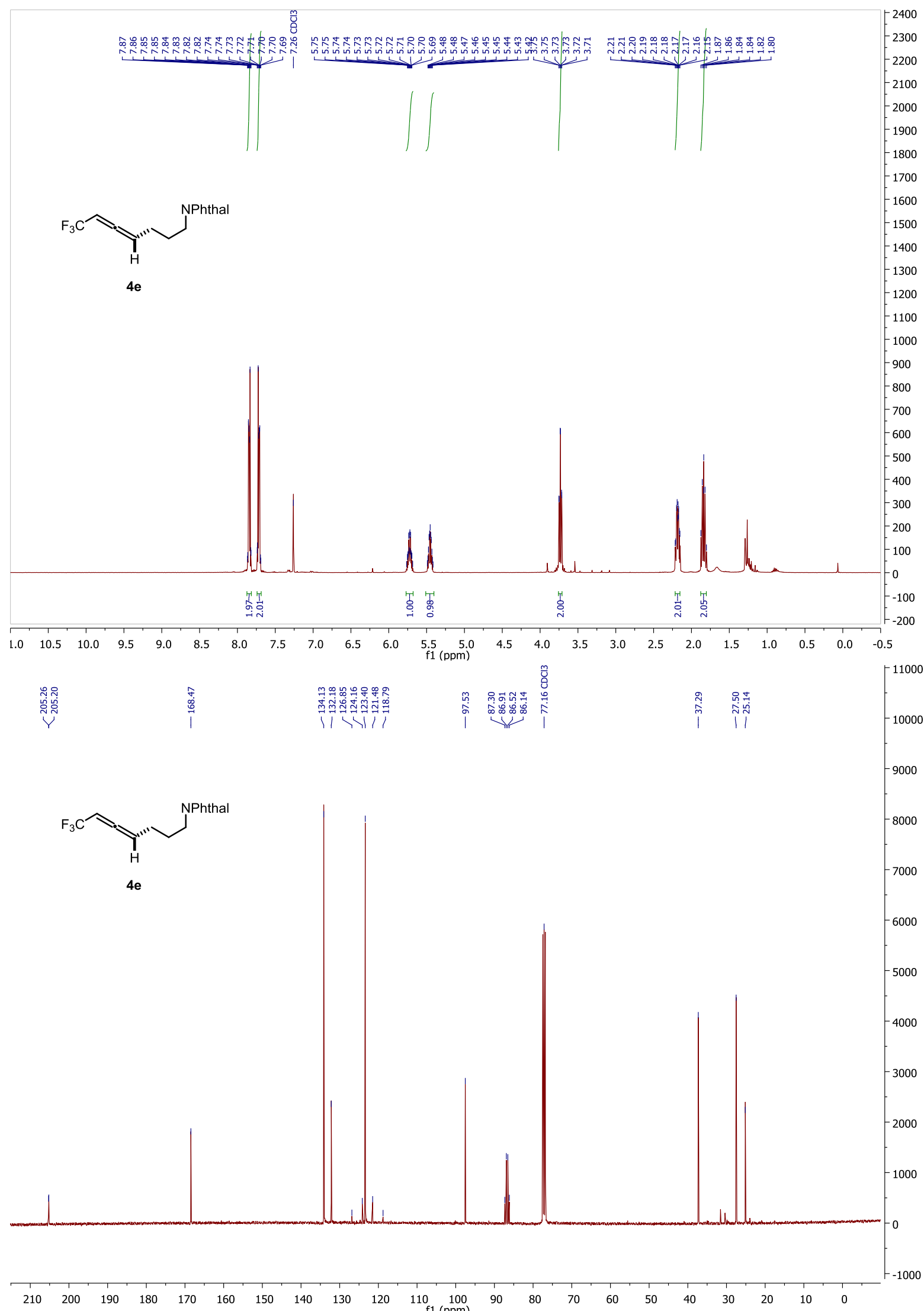




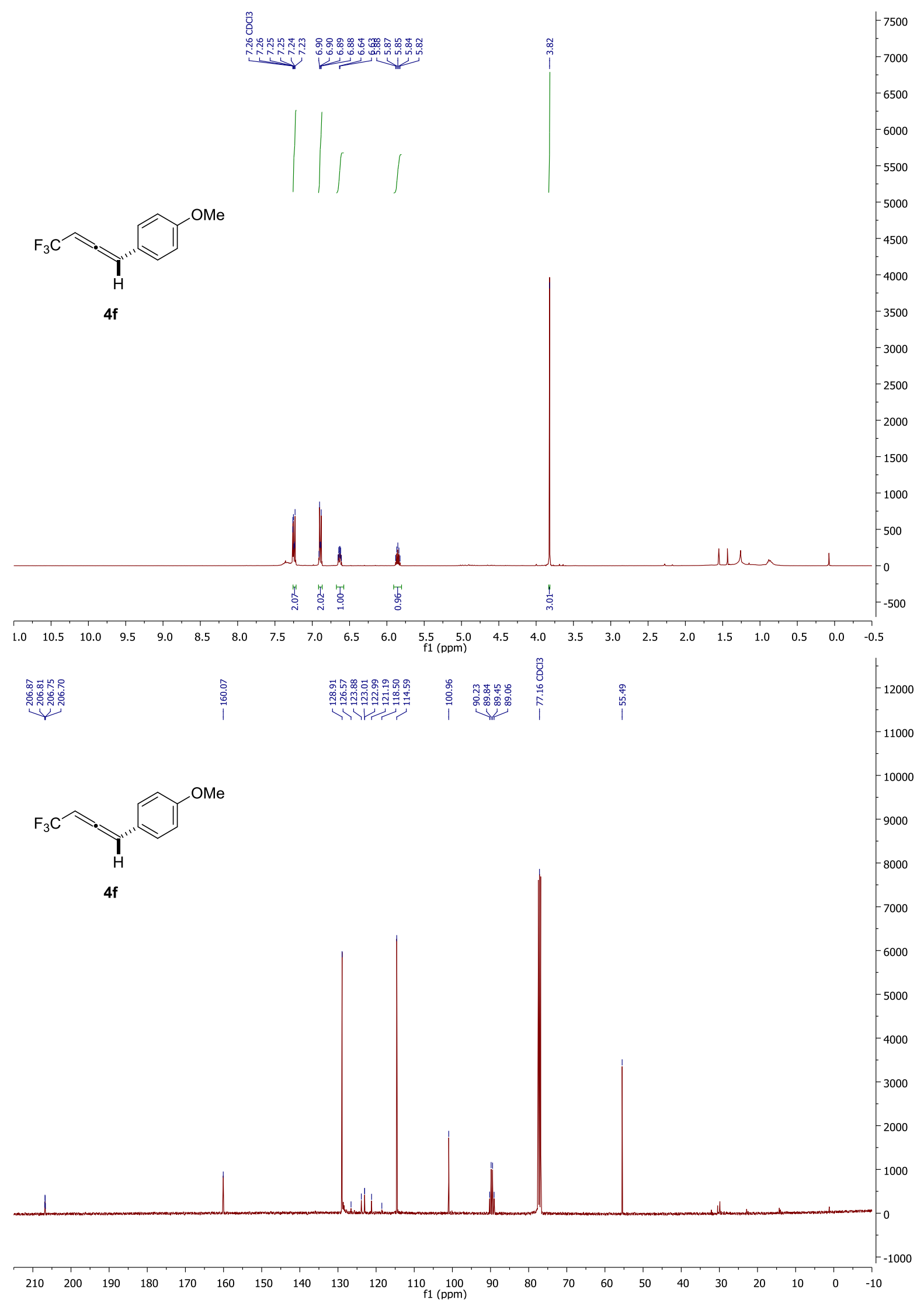




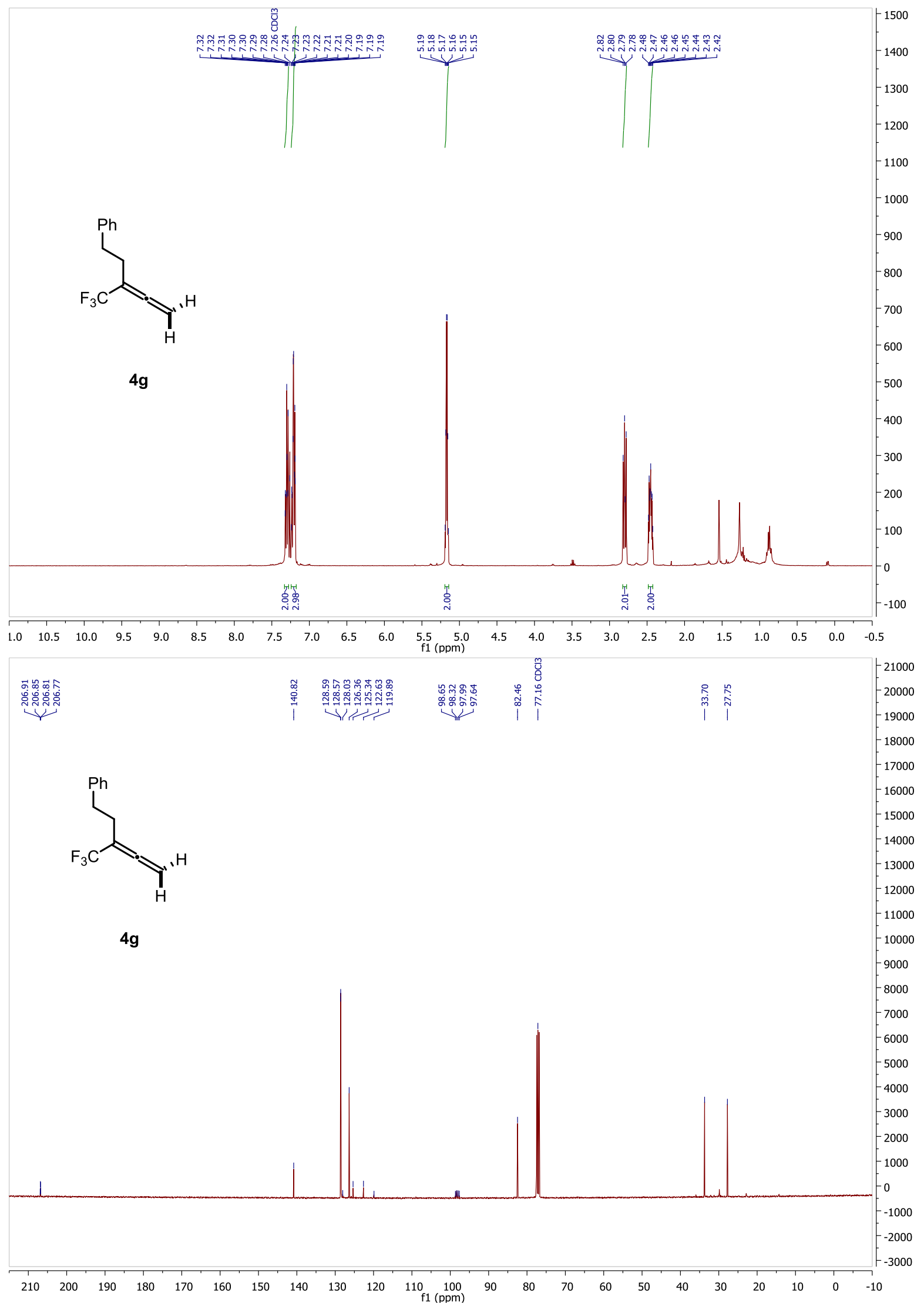



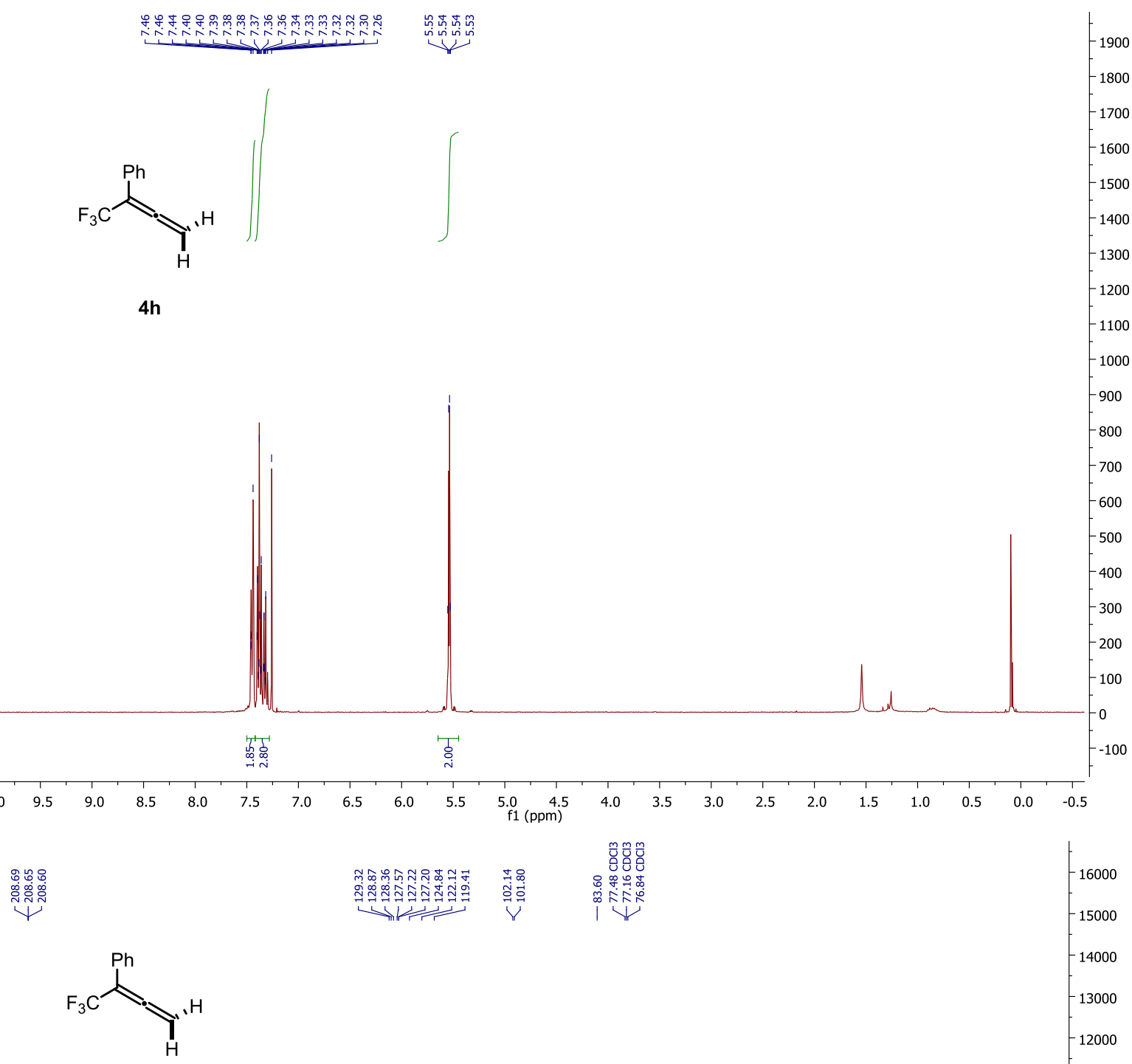

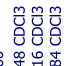

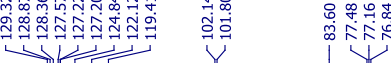

$-16000$

4h

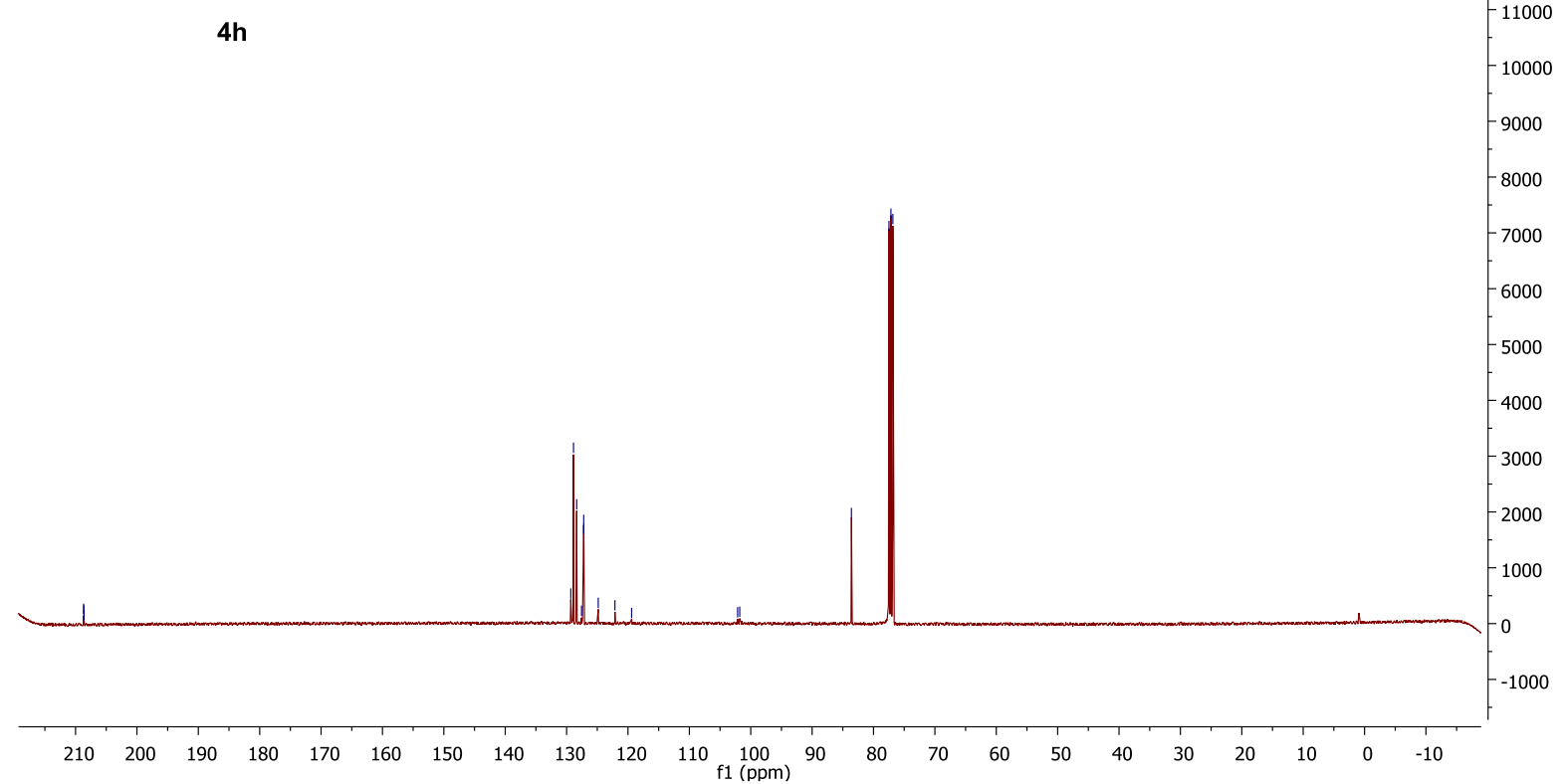




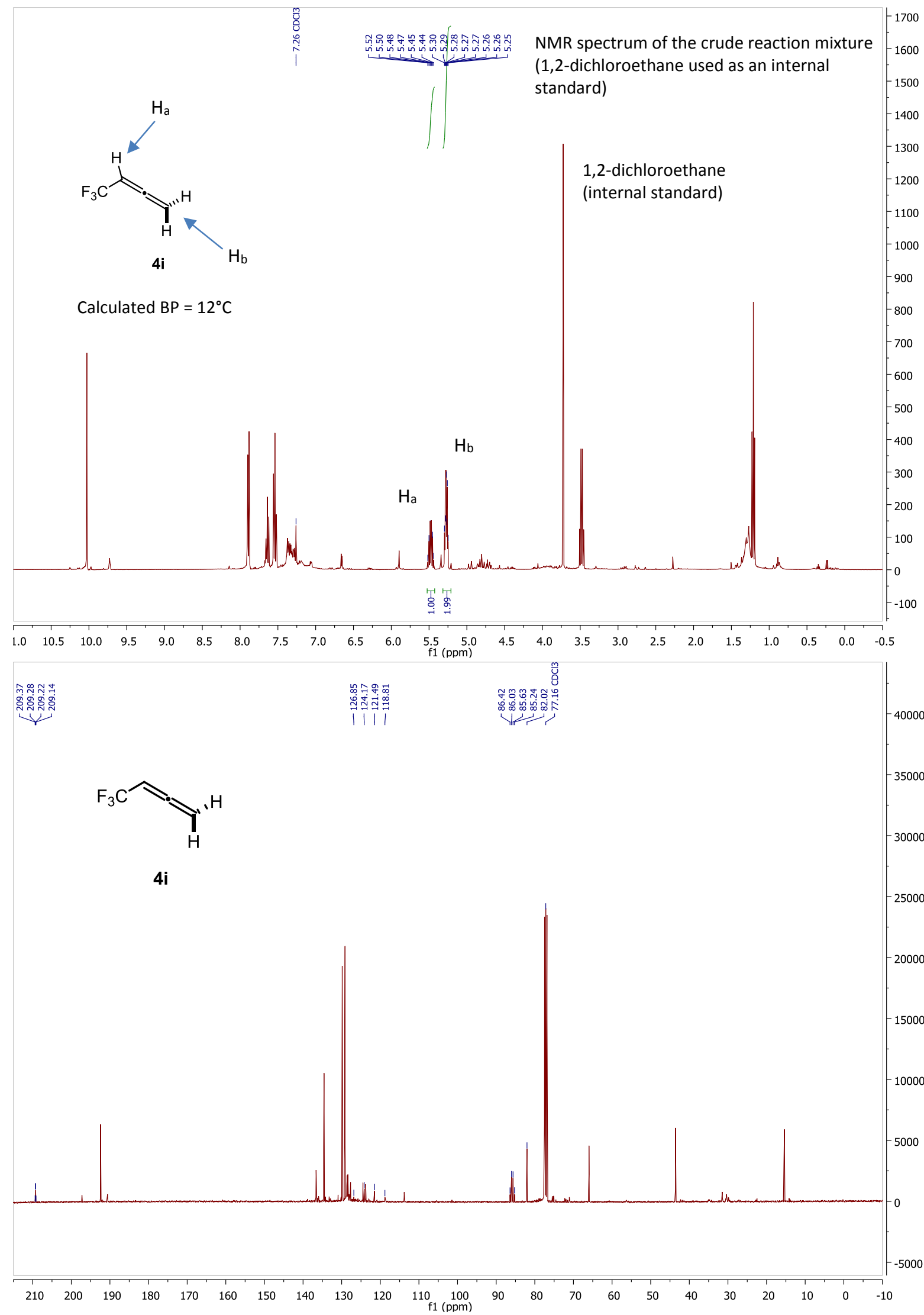



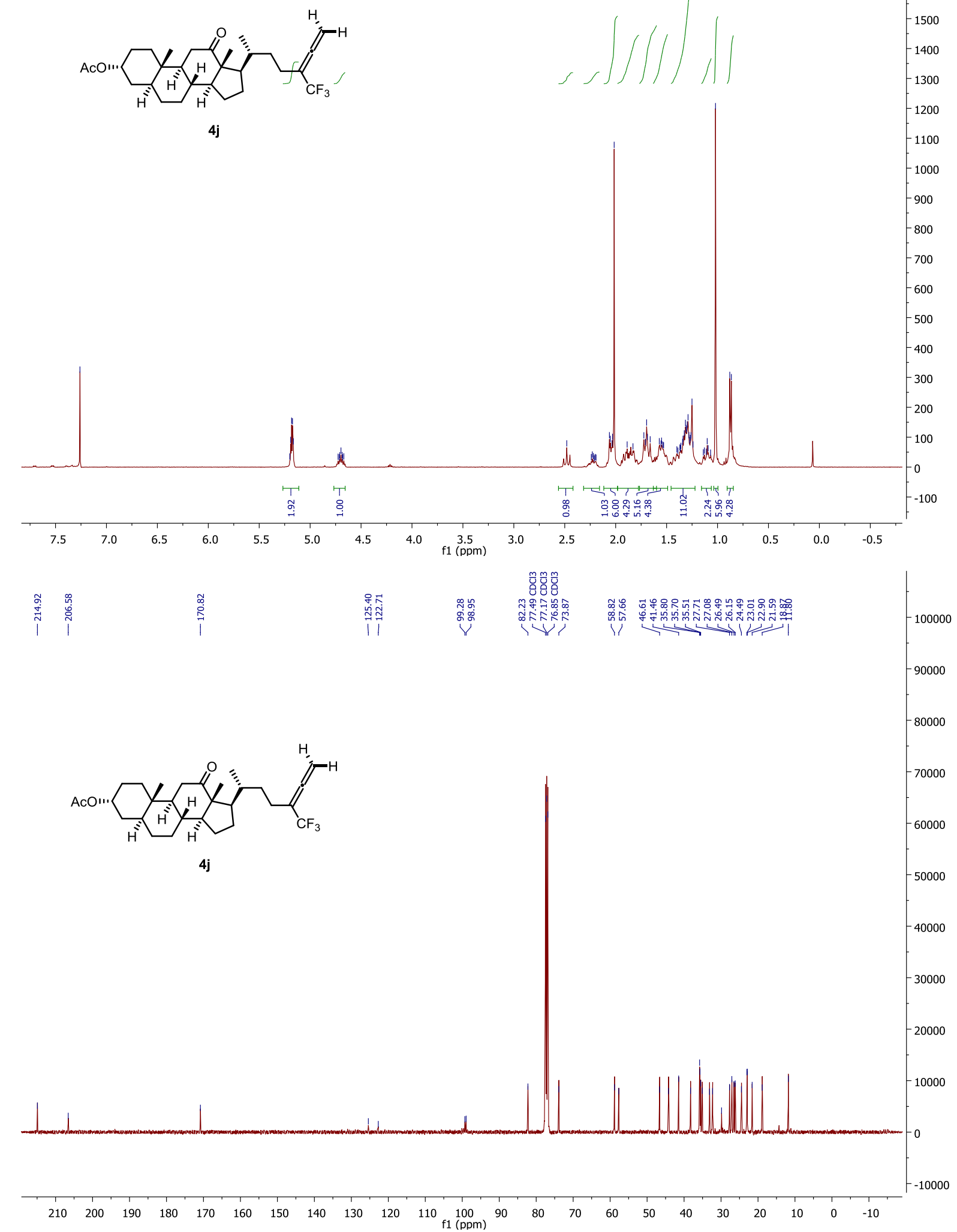


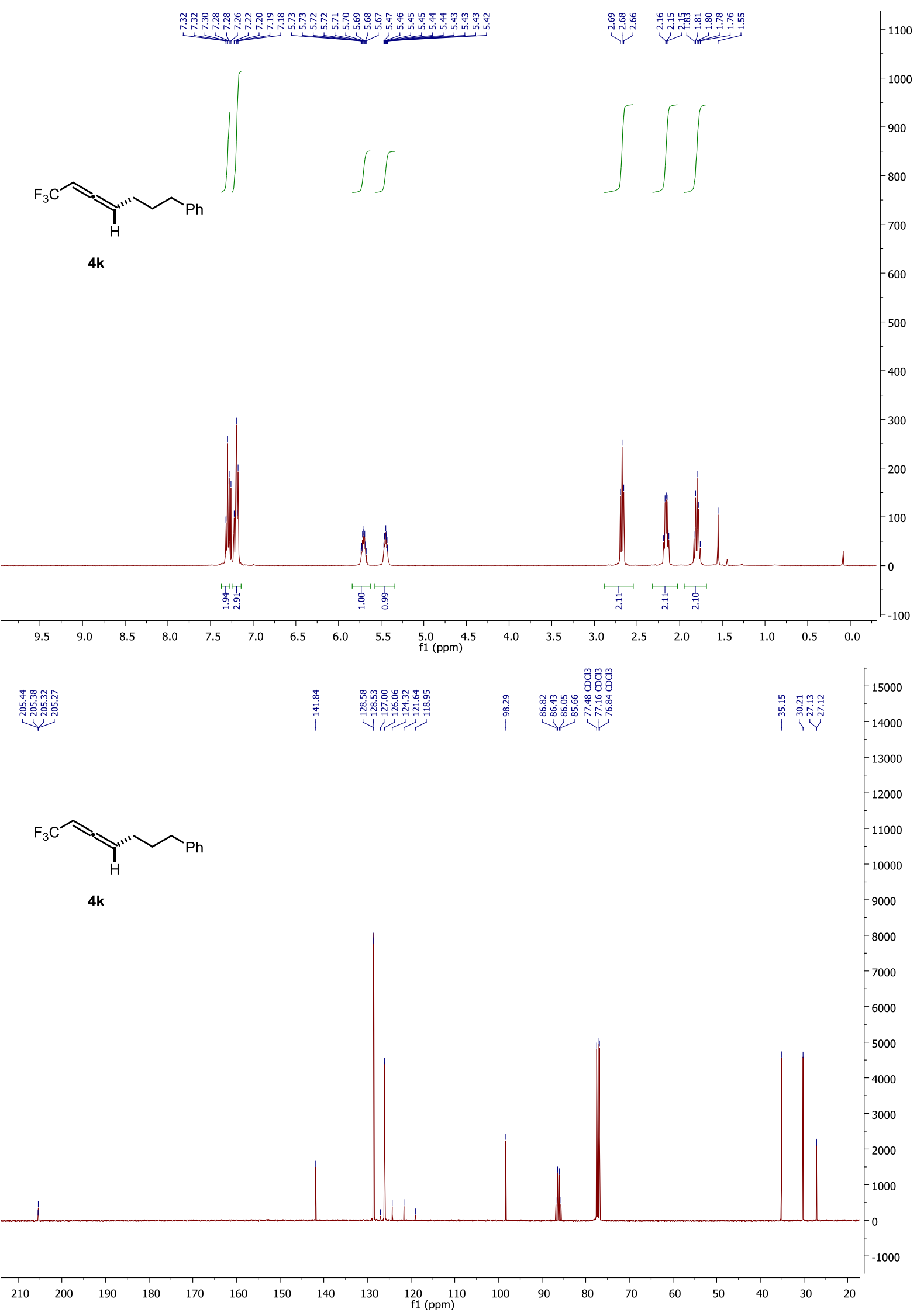




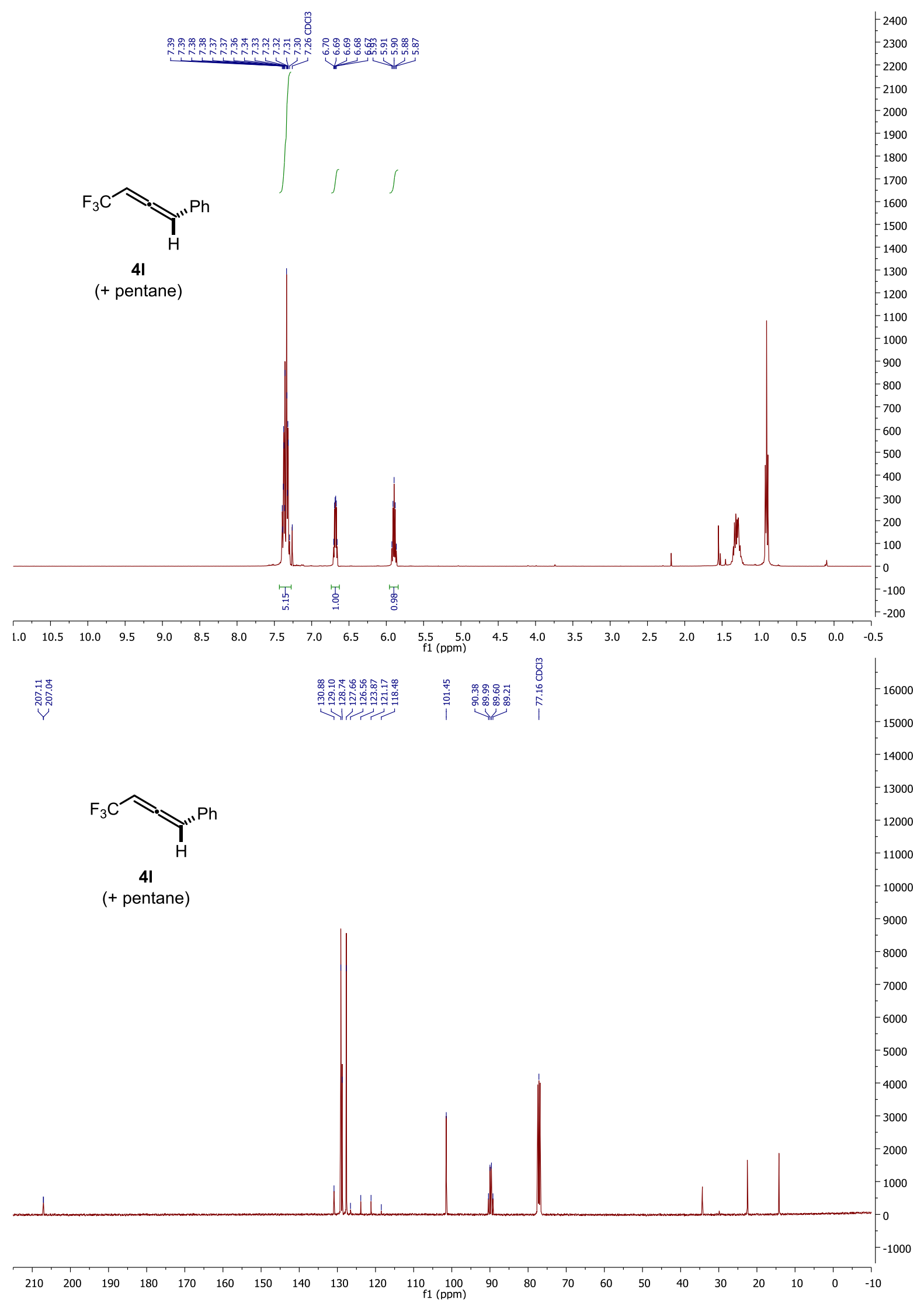




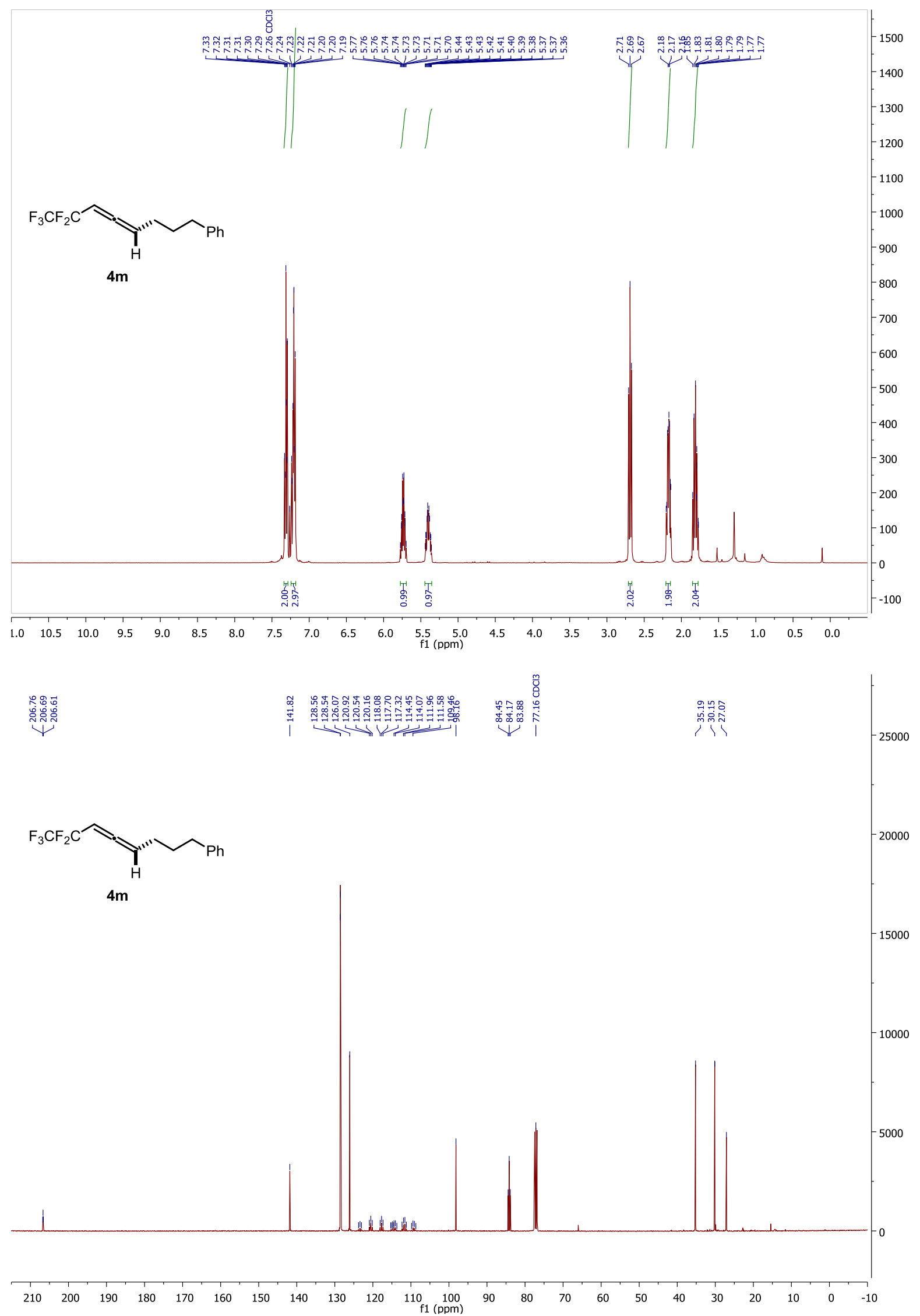




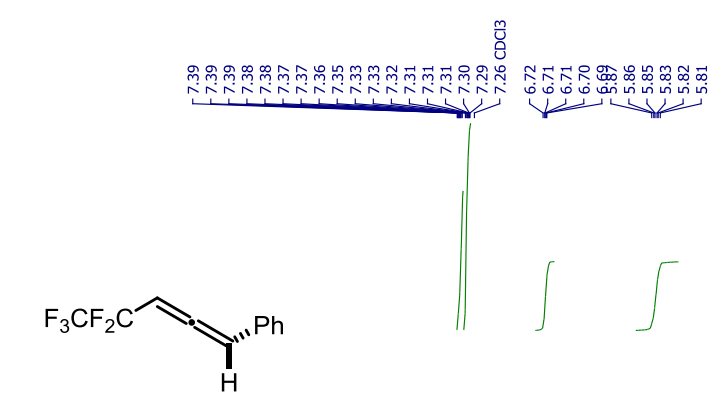

$4 n$

(+ pentane)
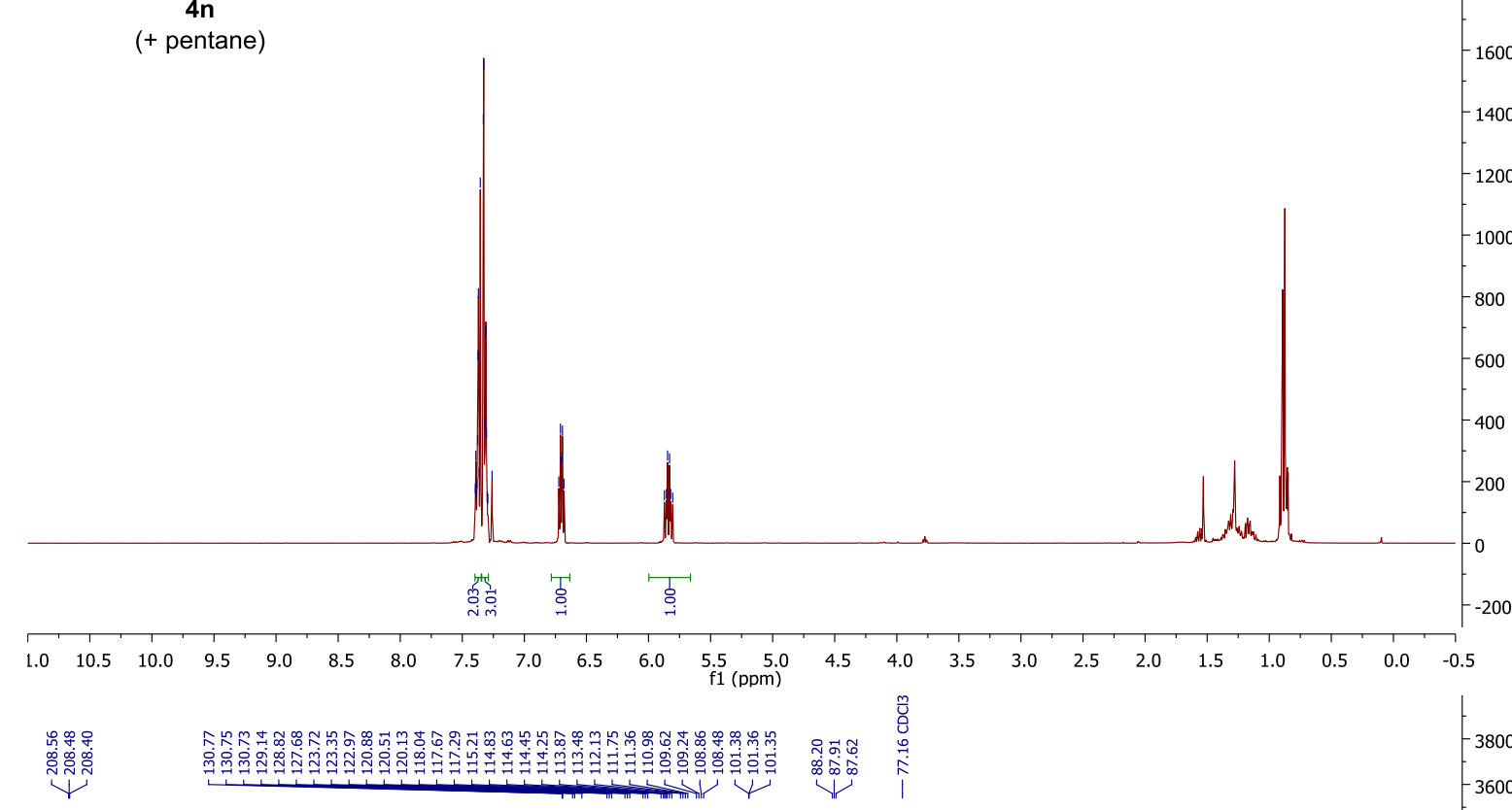

$\mathrm{F}_{3} \mathrm{CF}_{2} \mathrm{C} \overbrace{\mathrm{H}}^{\mathrm{H}}$

$4 n$

(+ pentane)

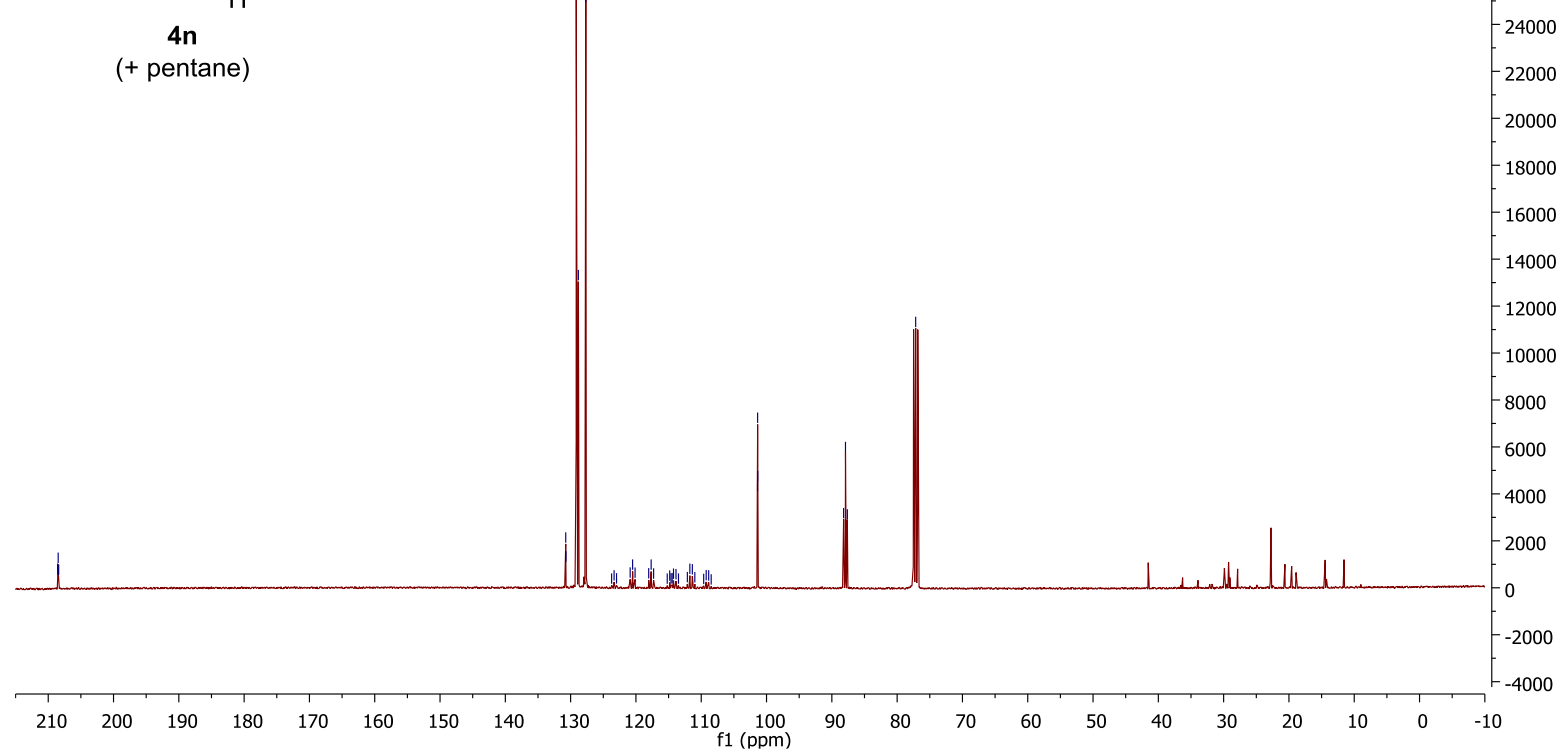




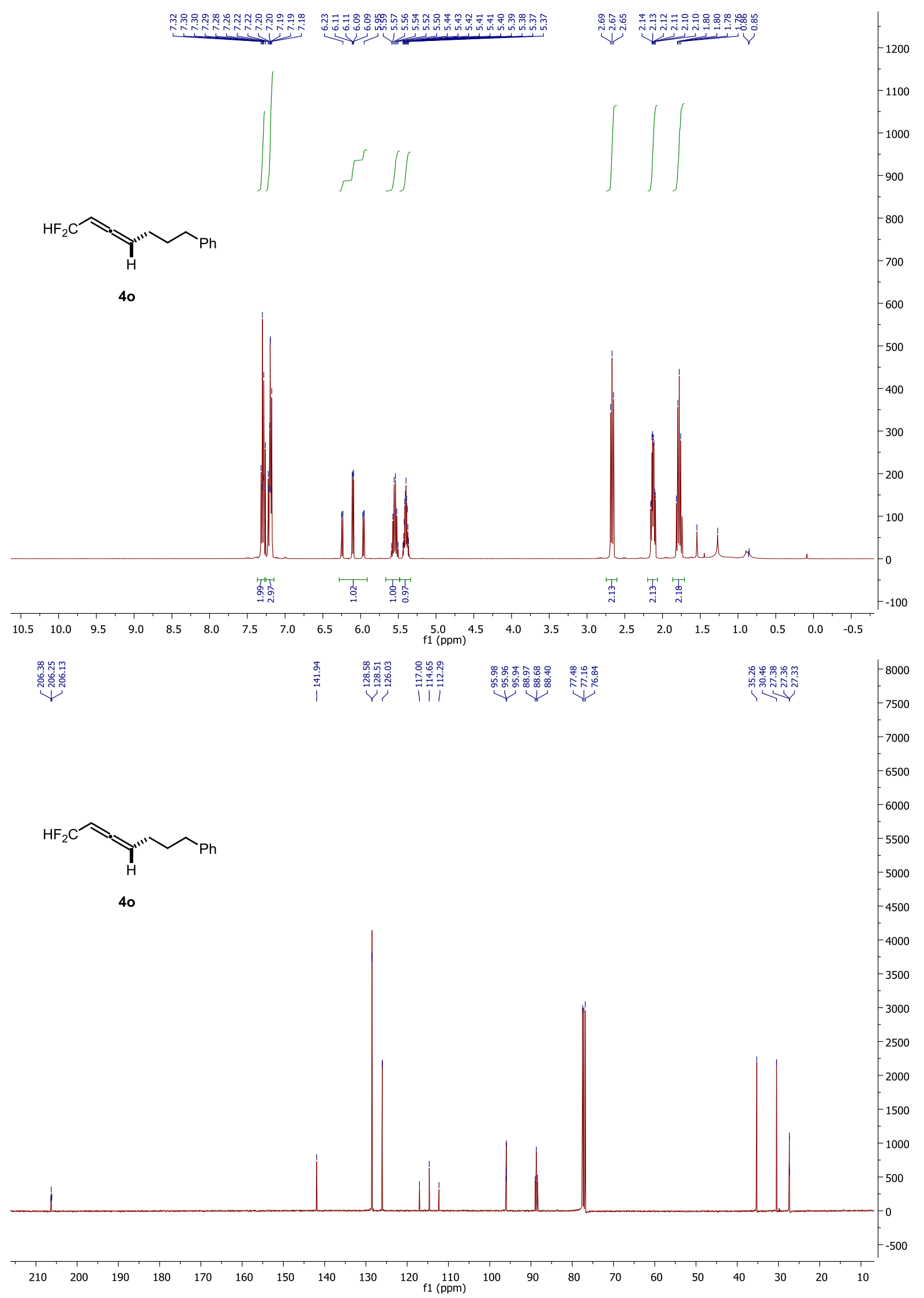




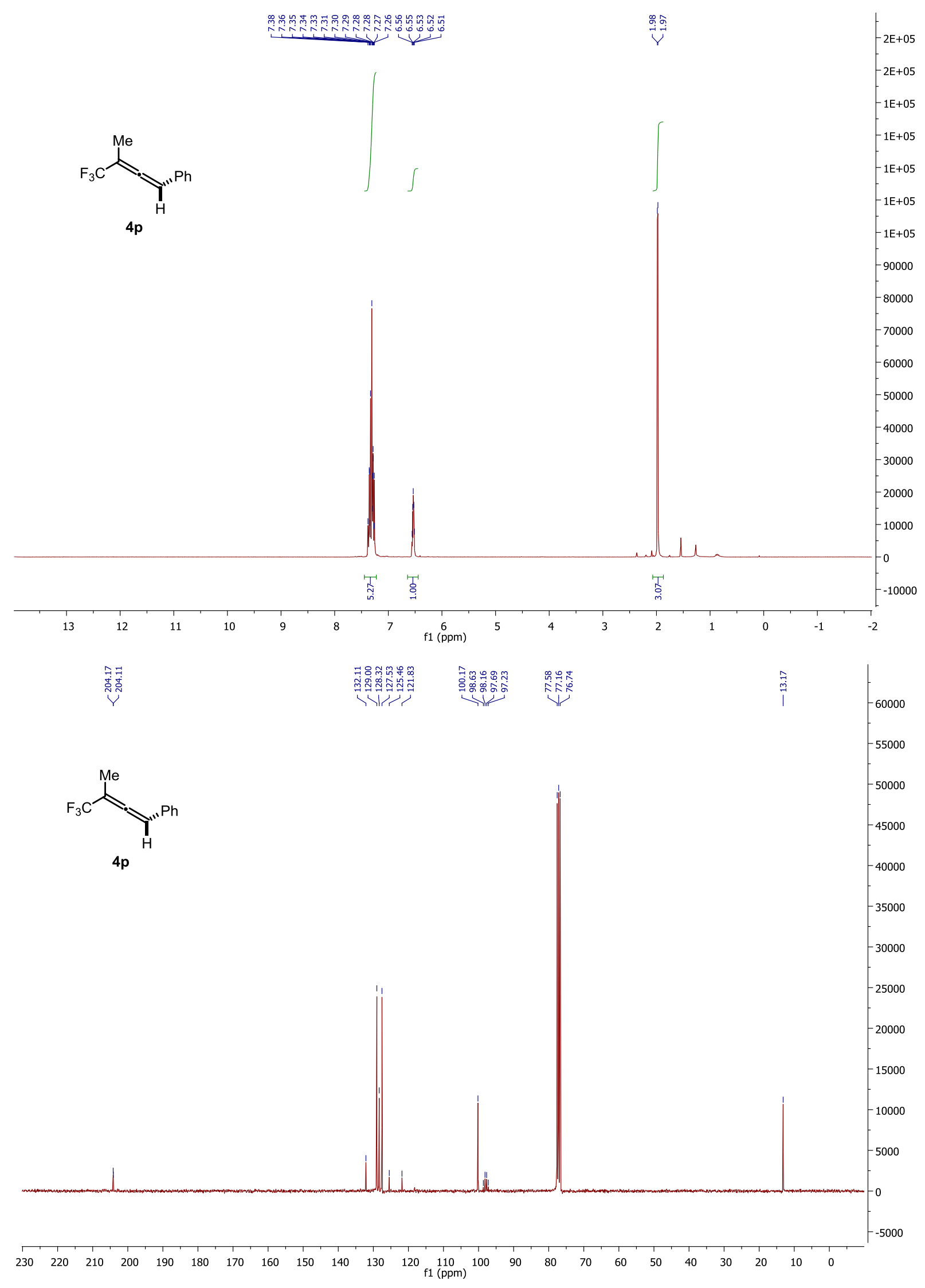




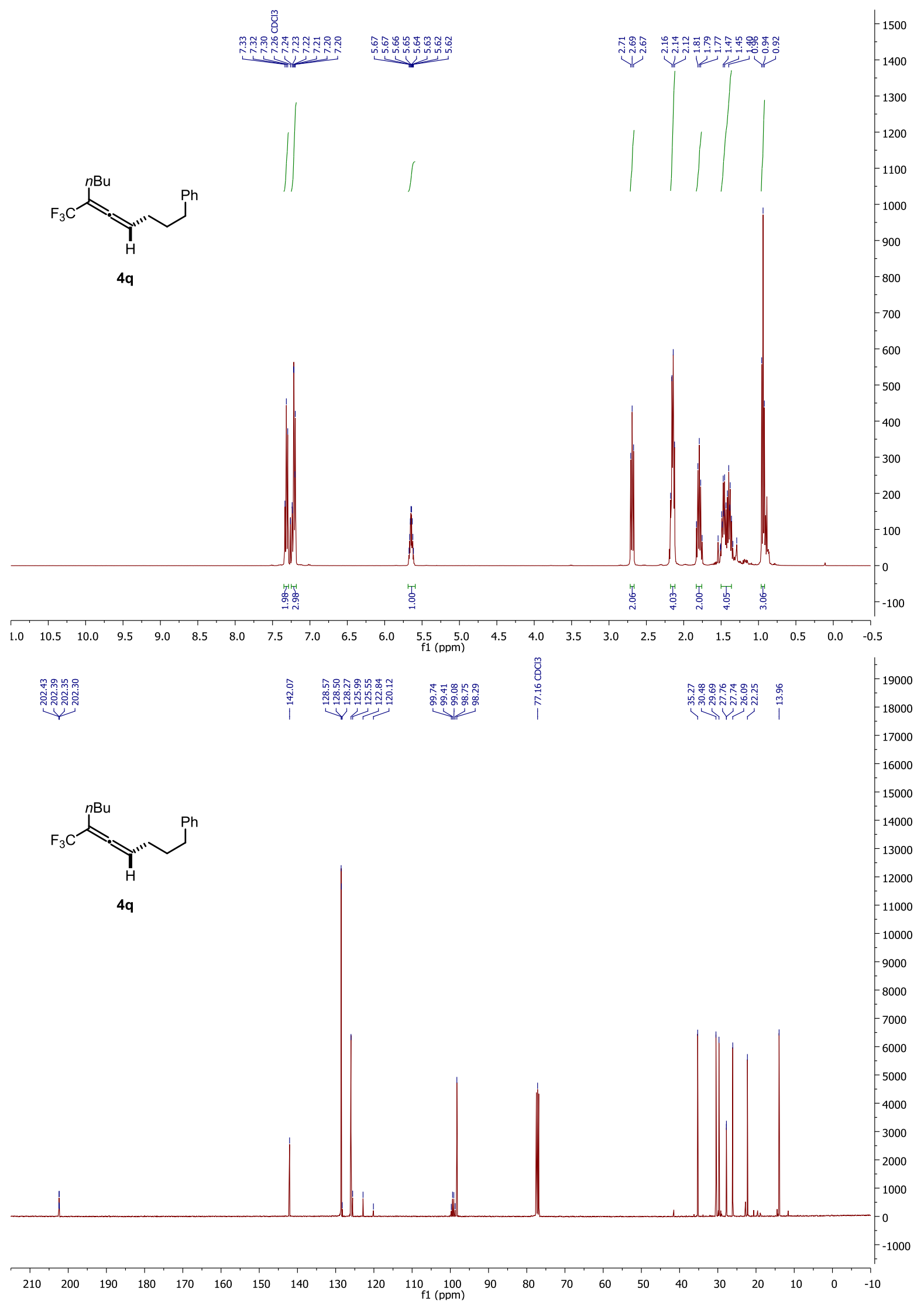




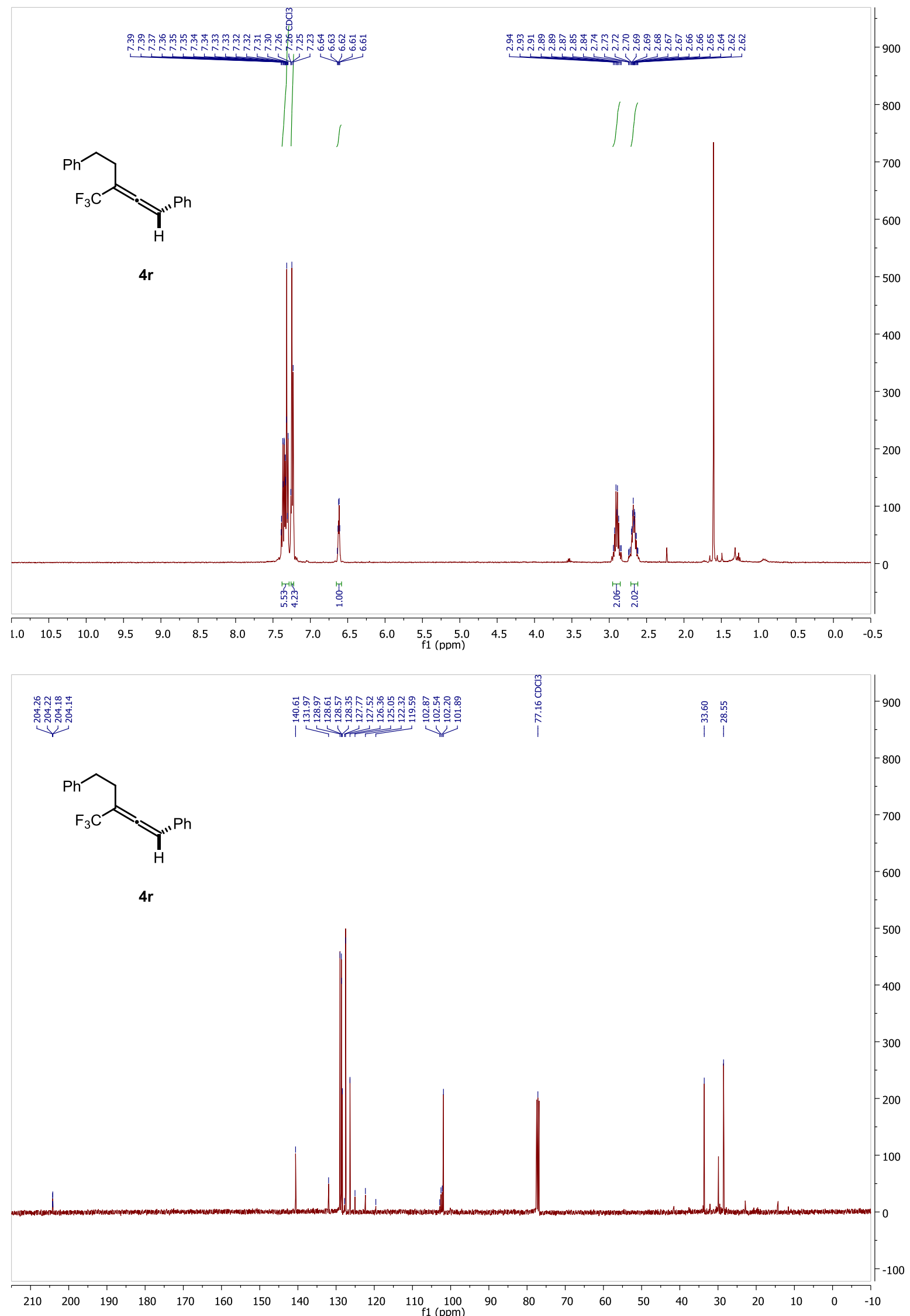



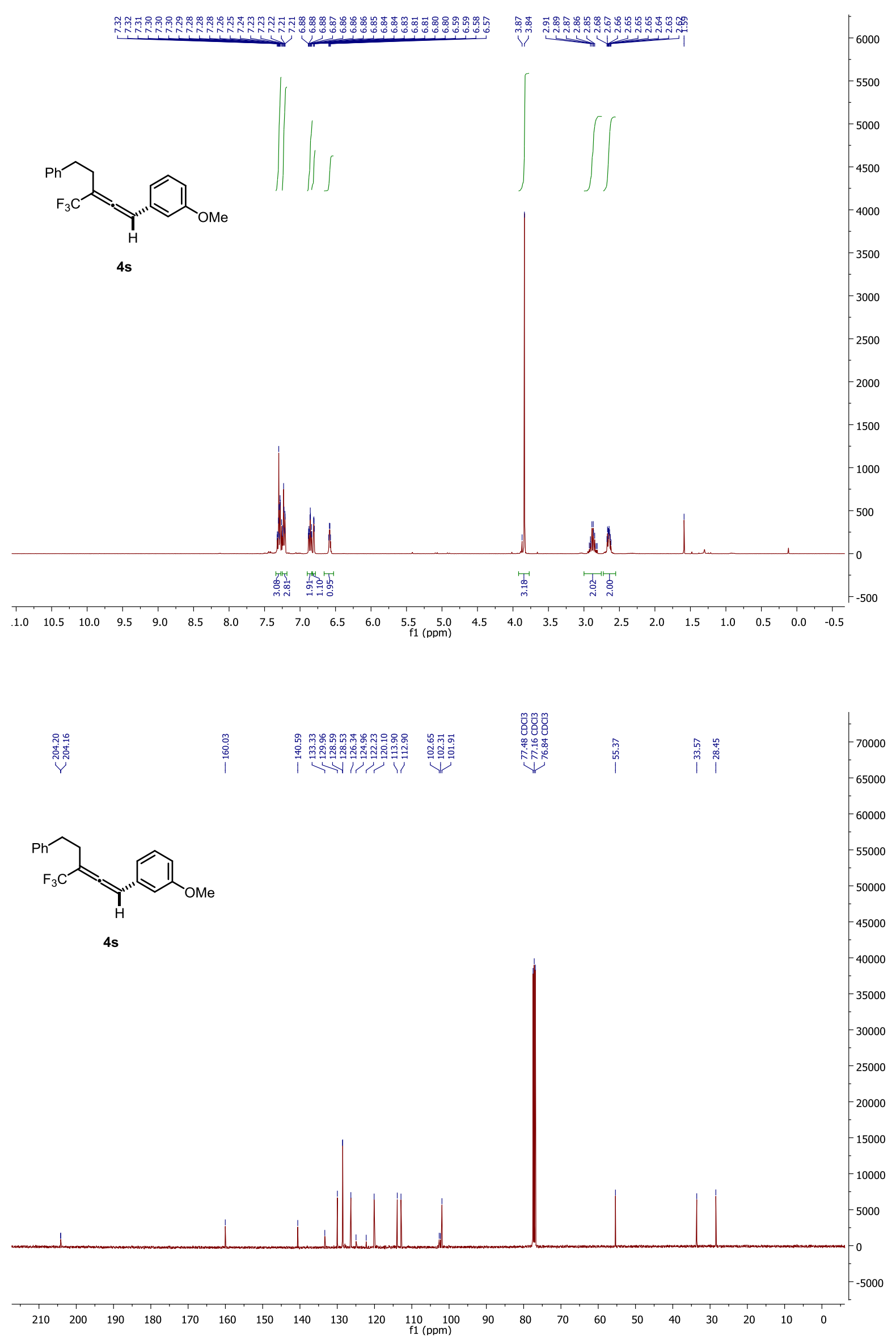


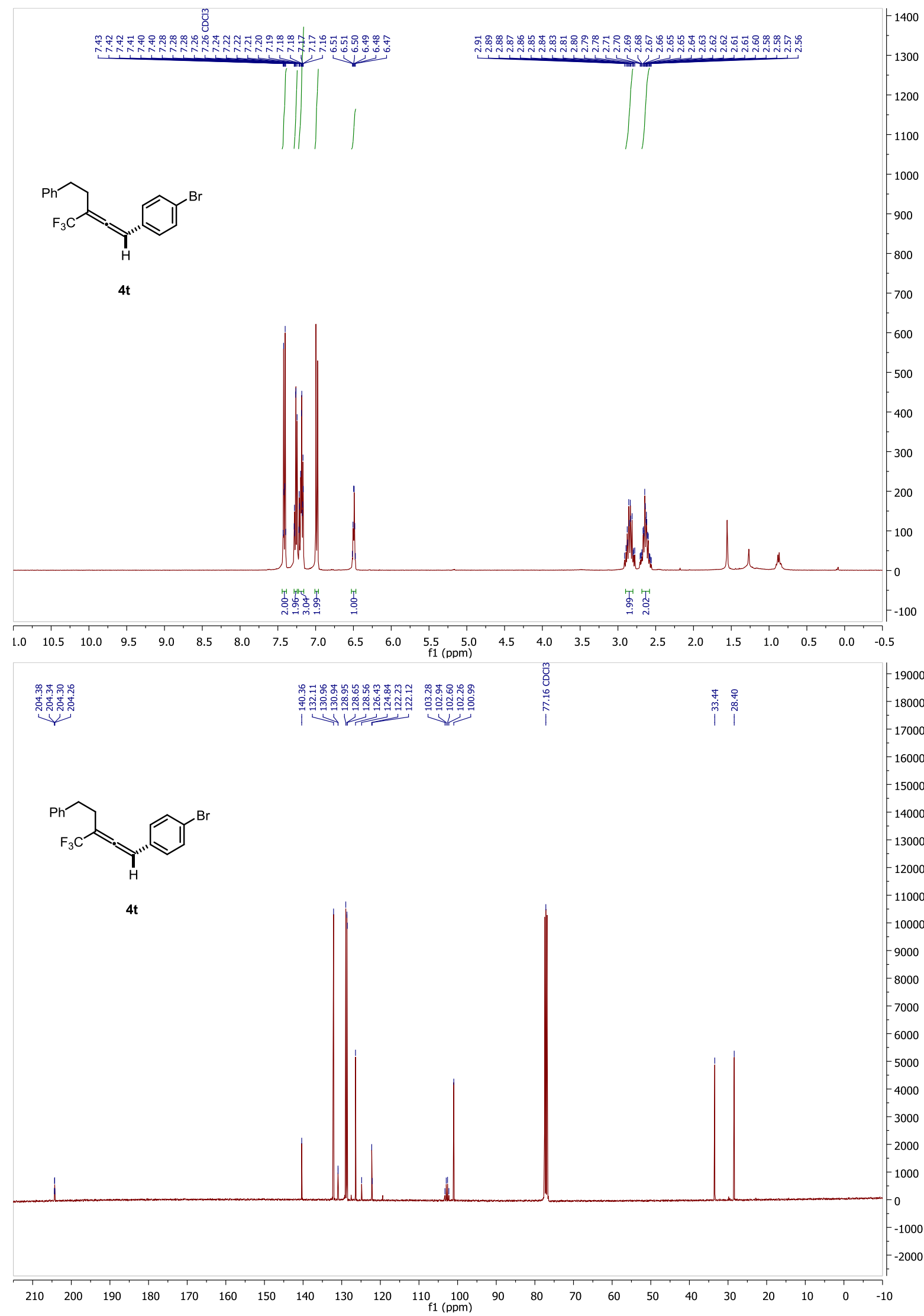




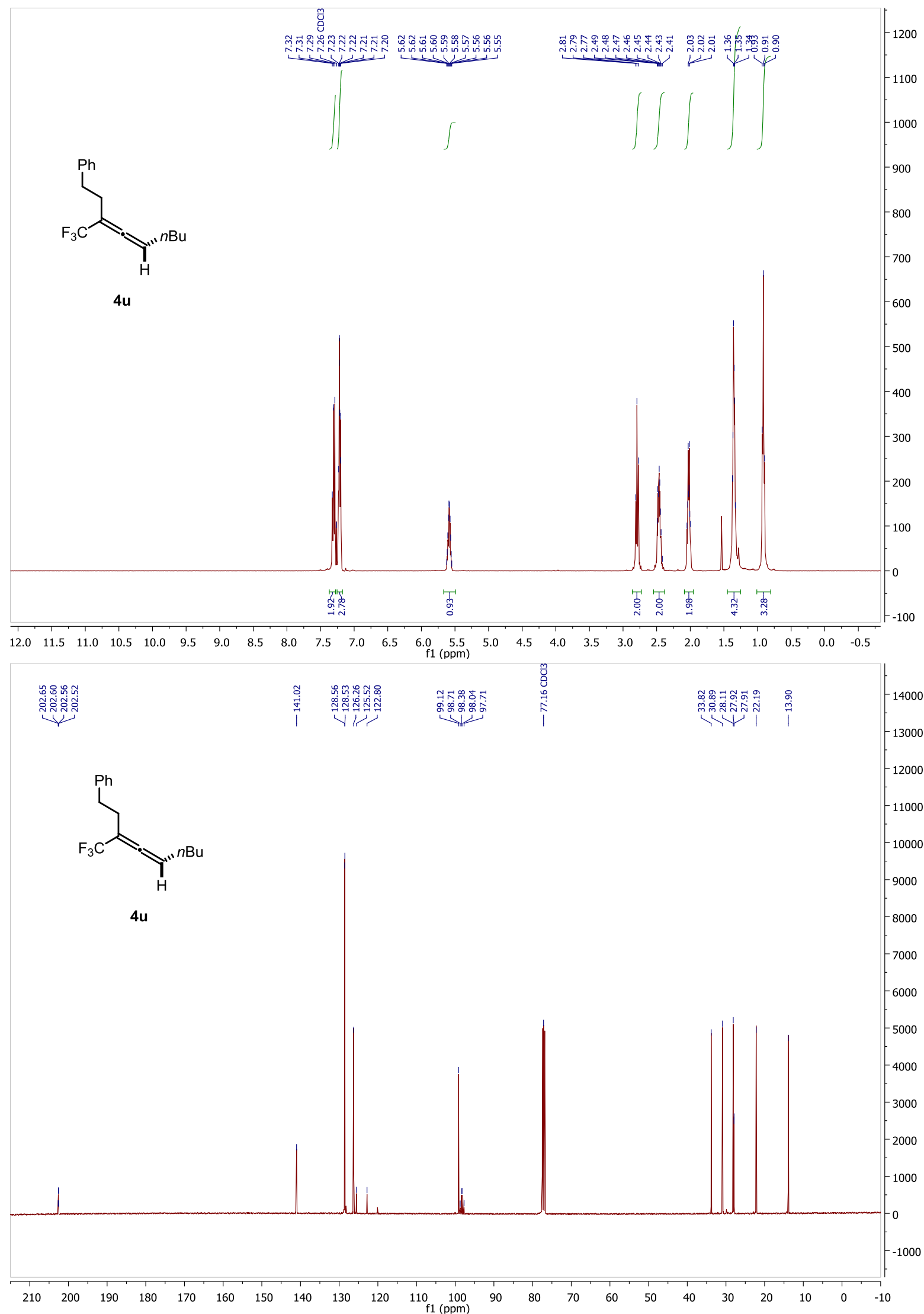




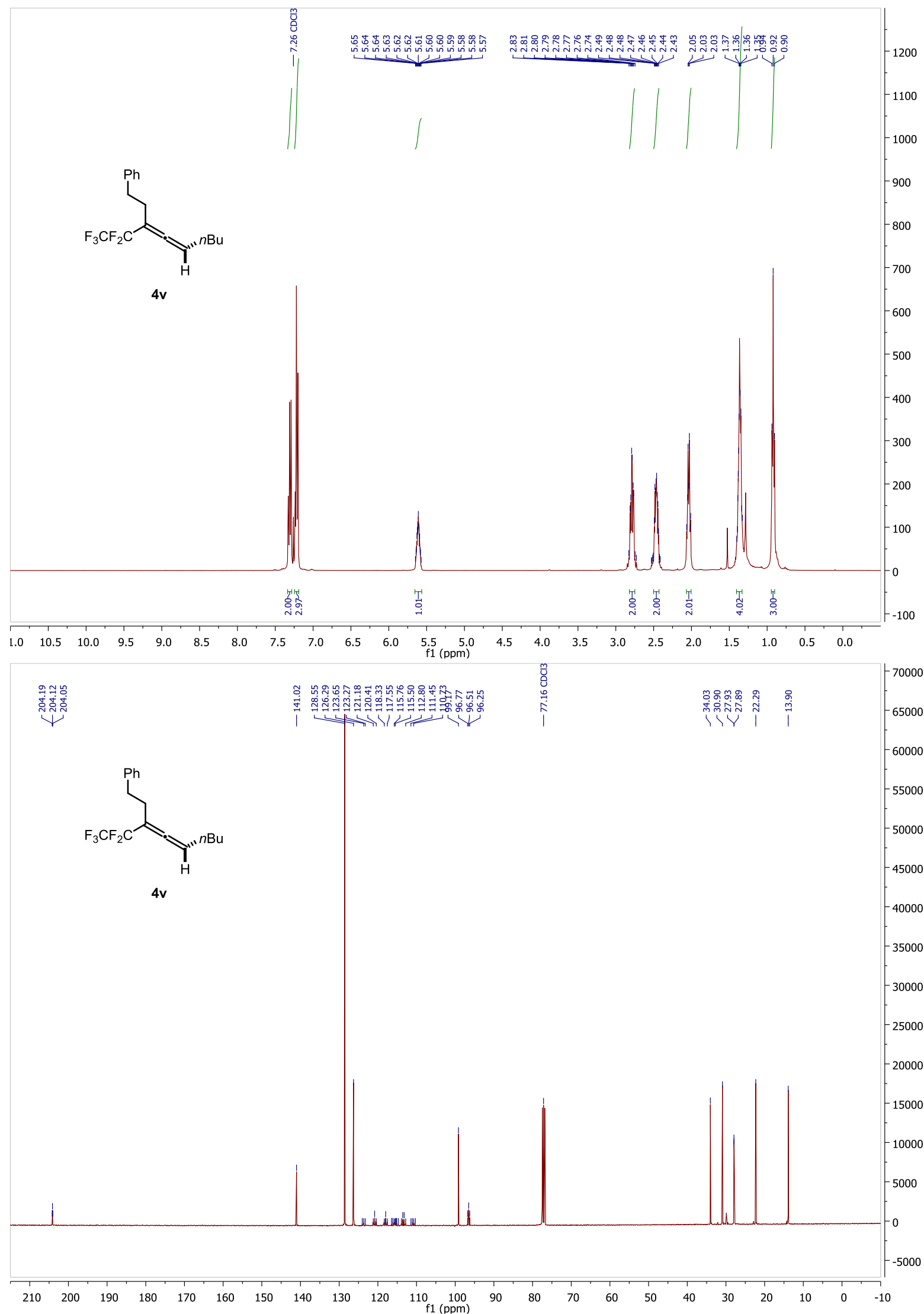




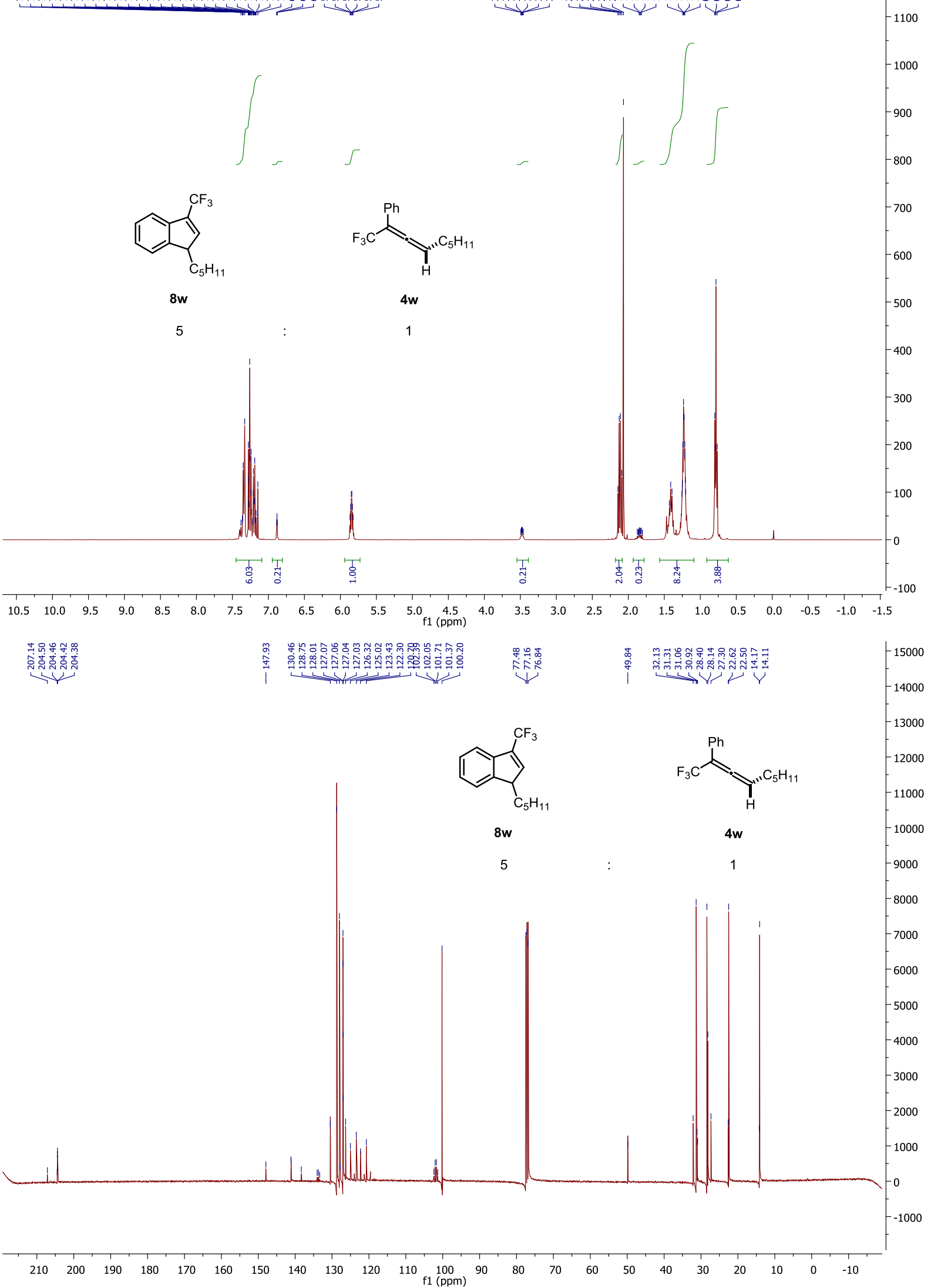



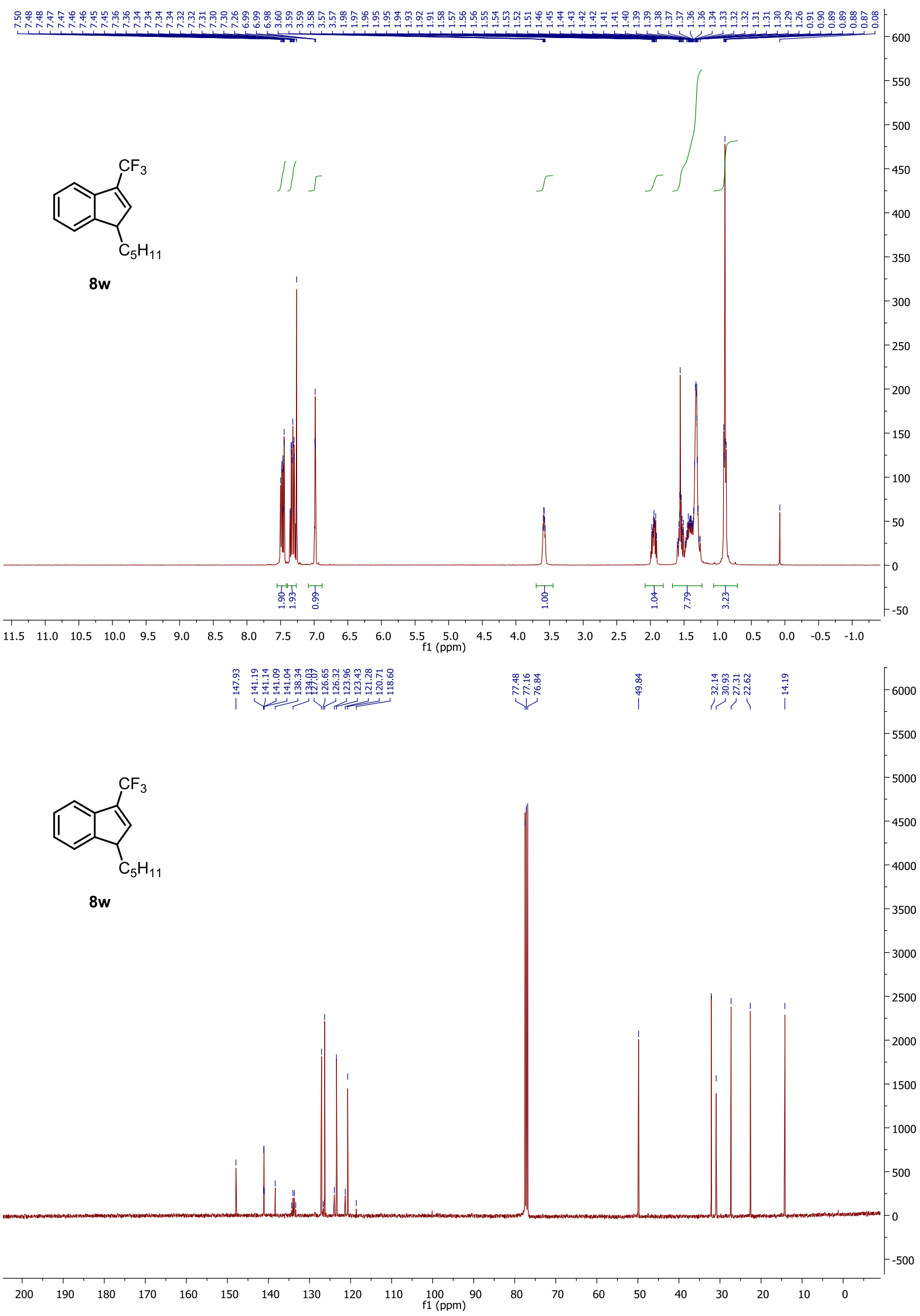
NMR monitoring for the formation of indene $\mathbf{8 x}$ :

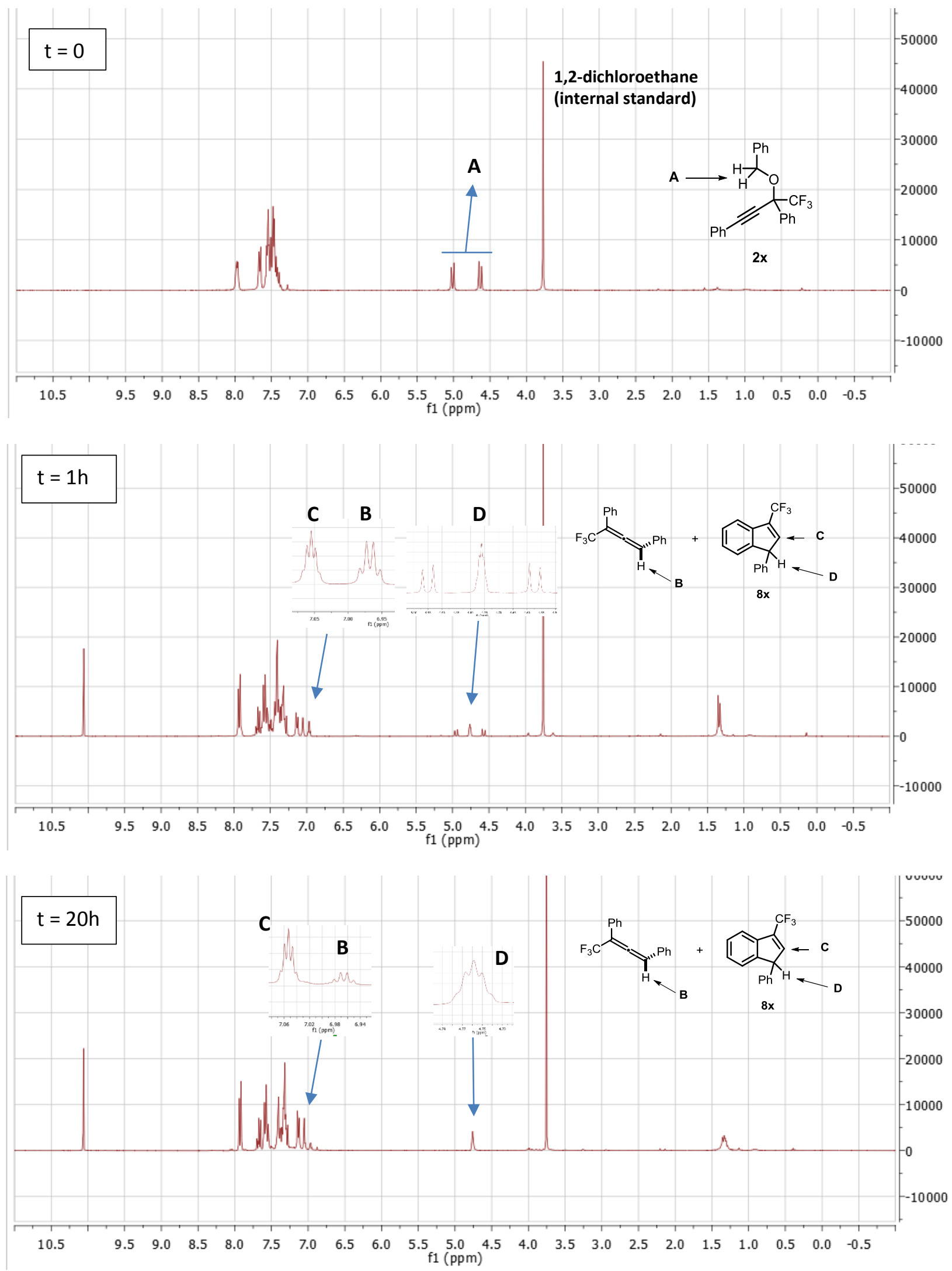




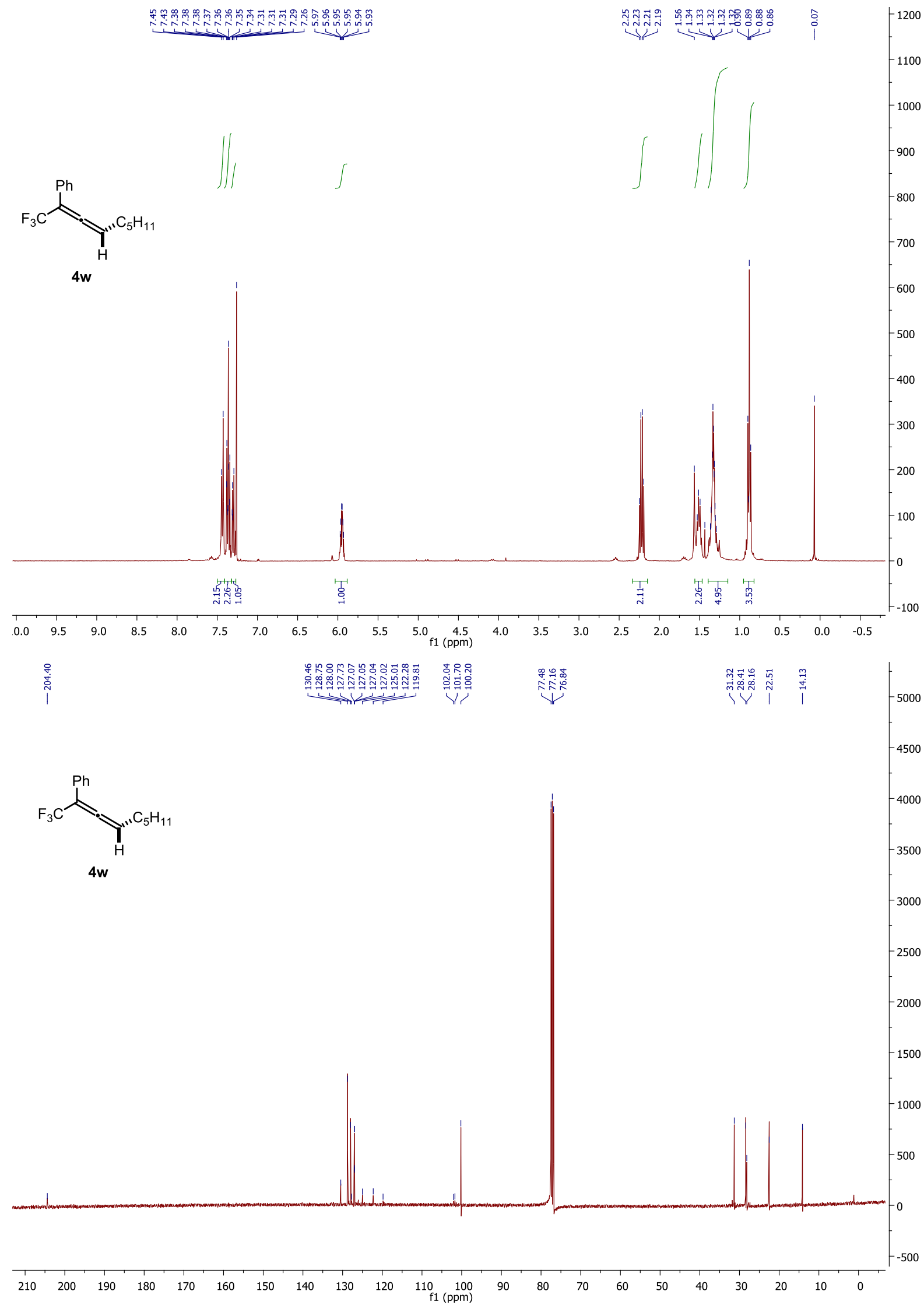




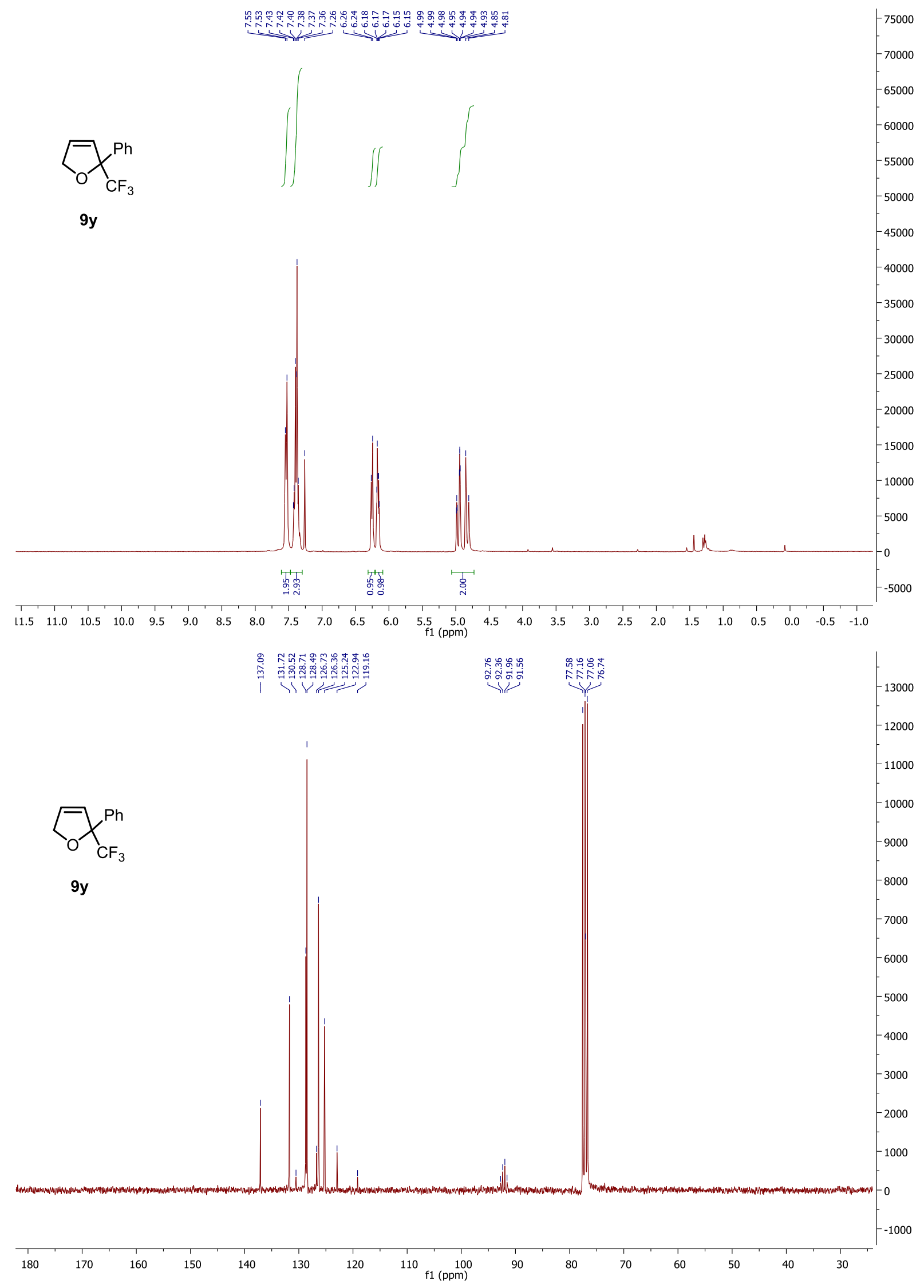




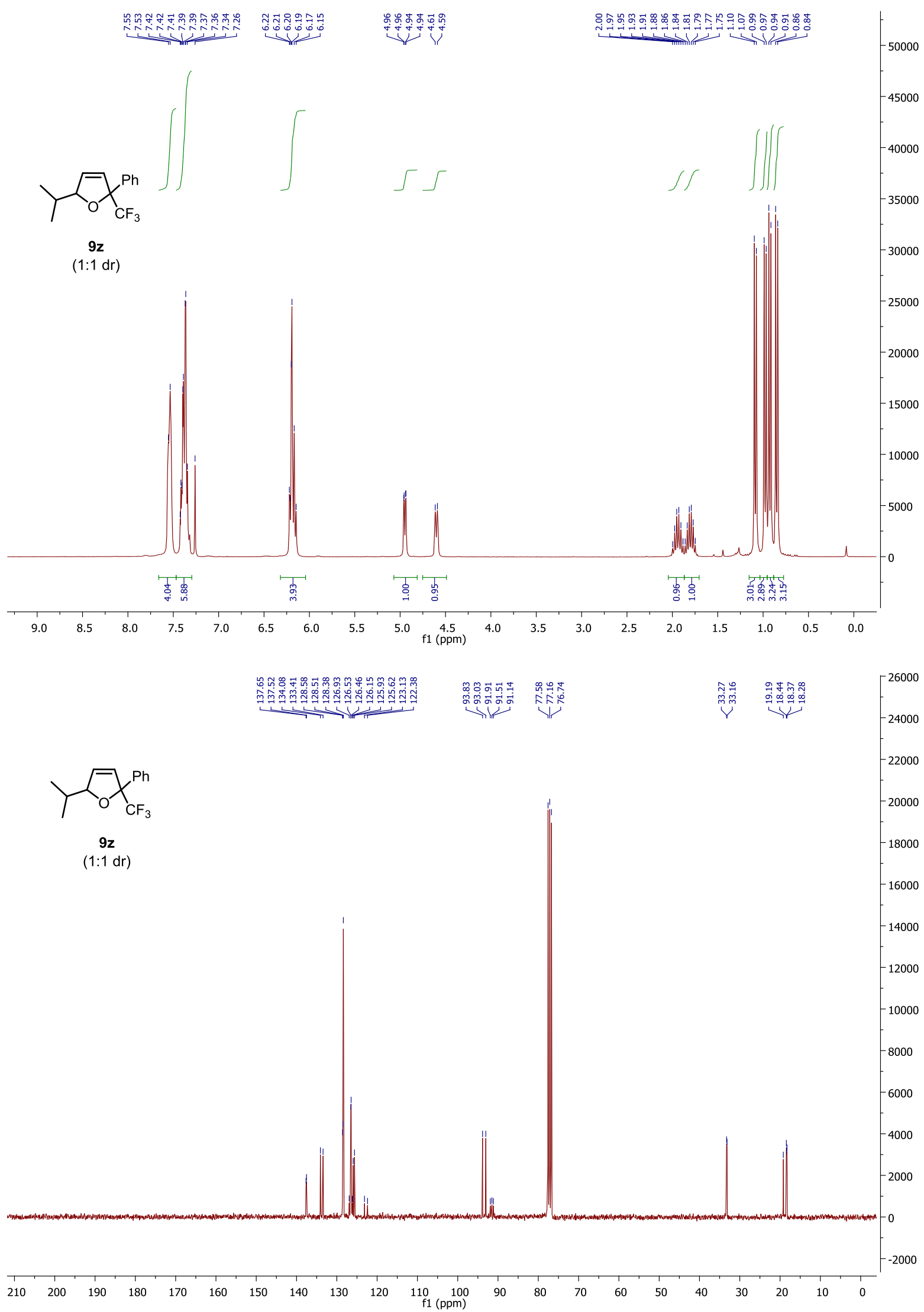




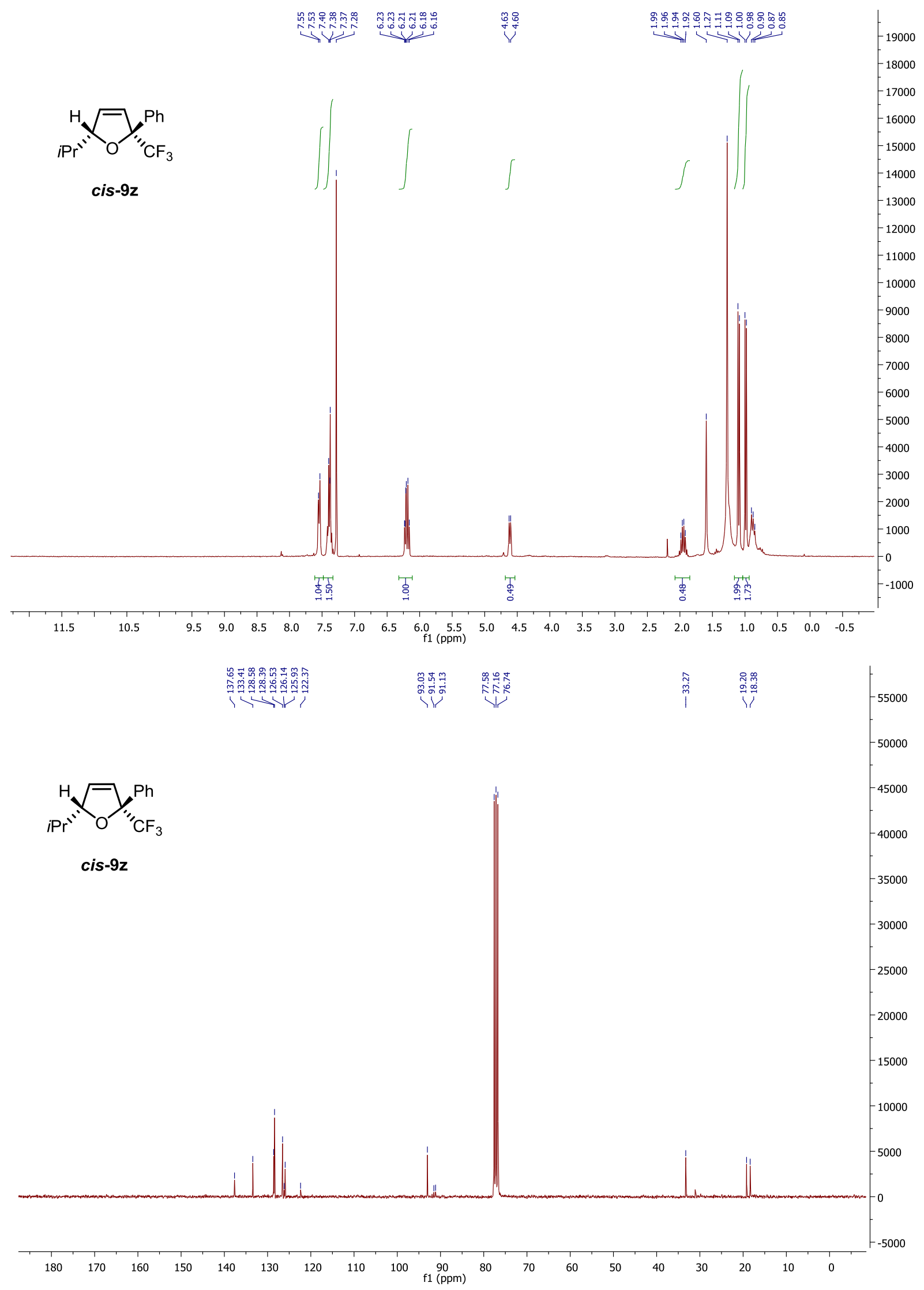




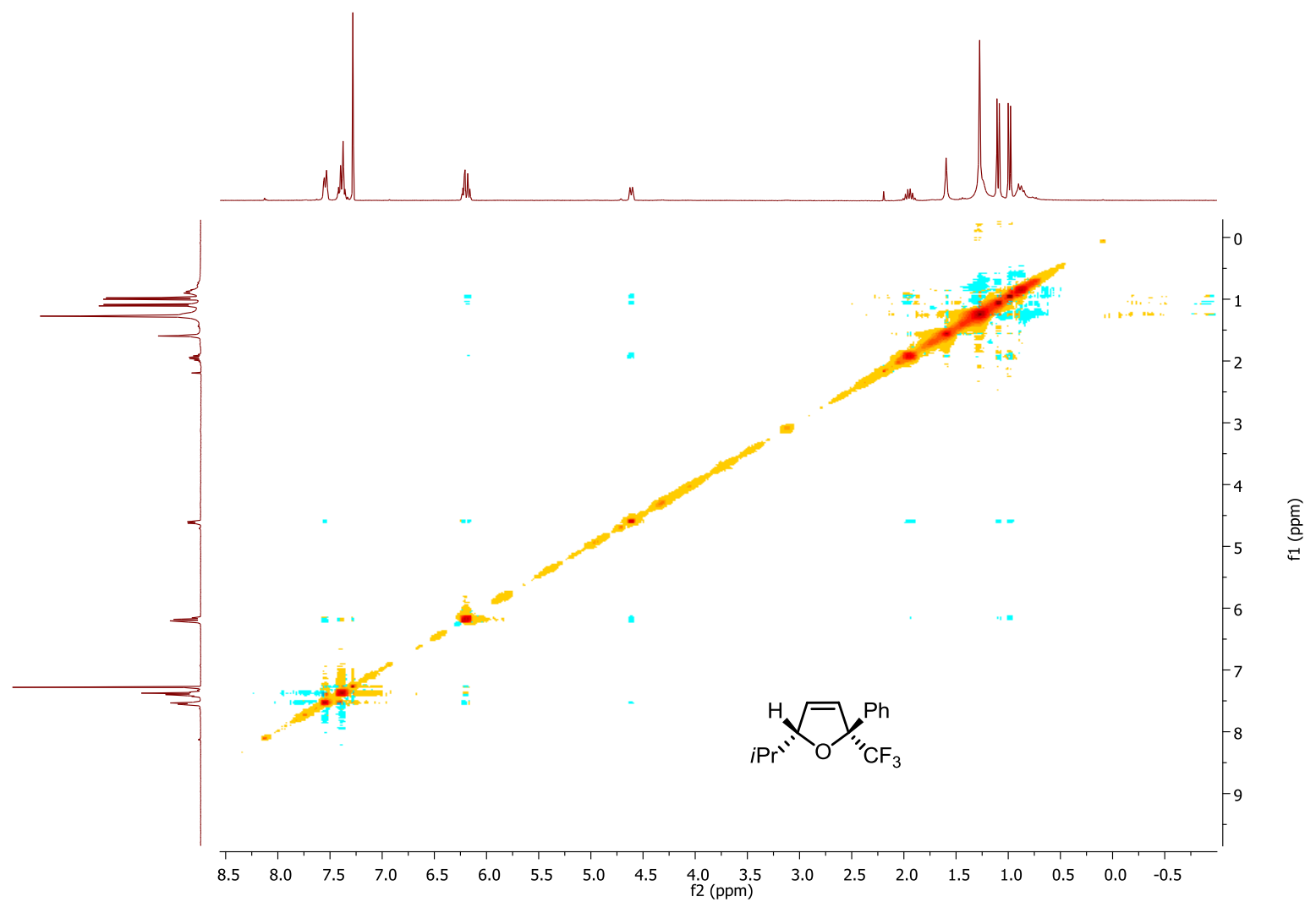




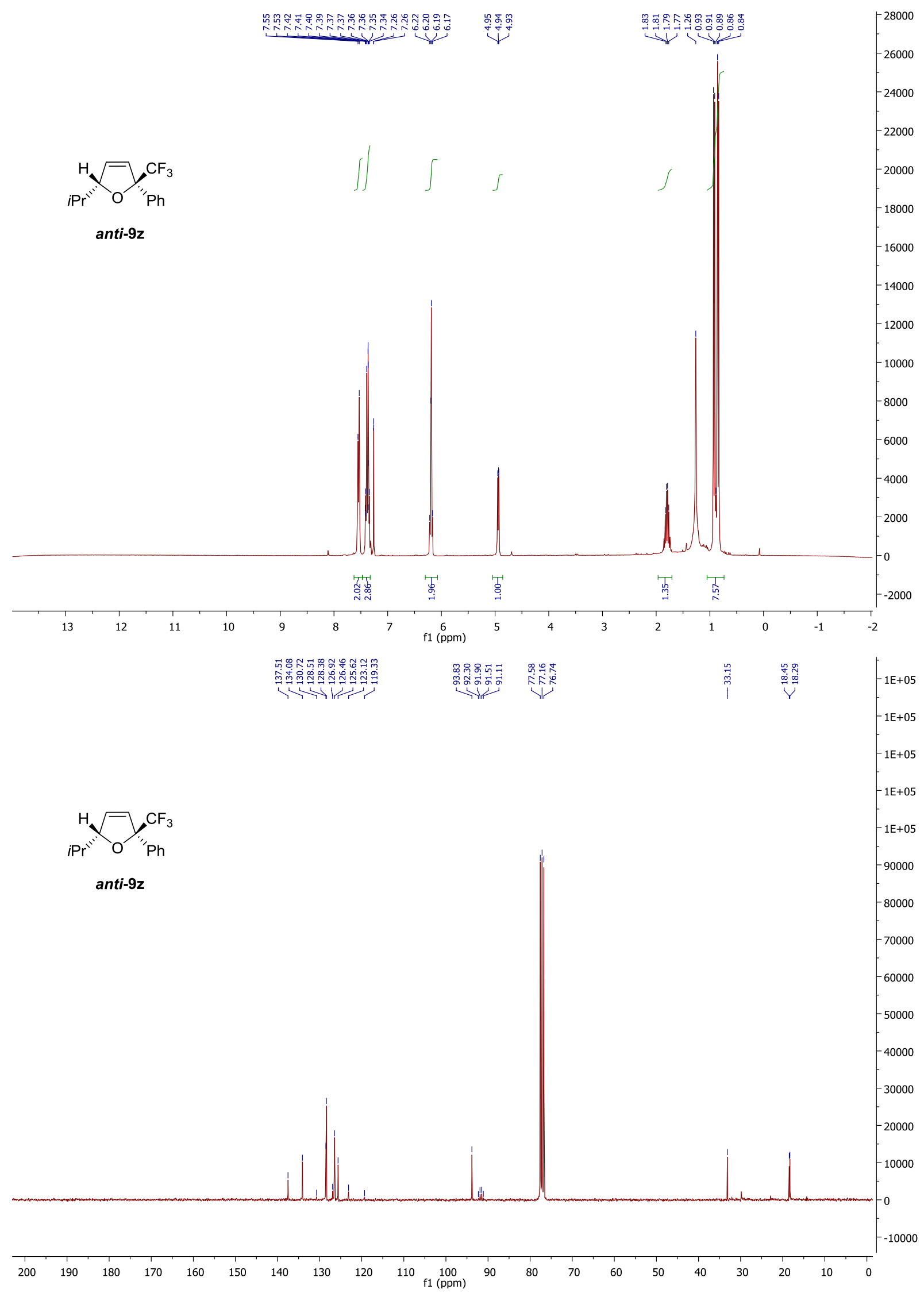




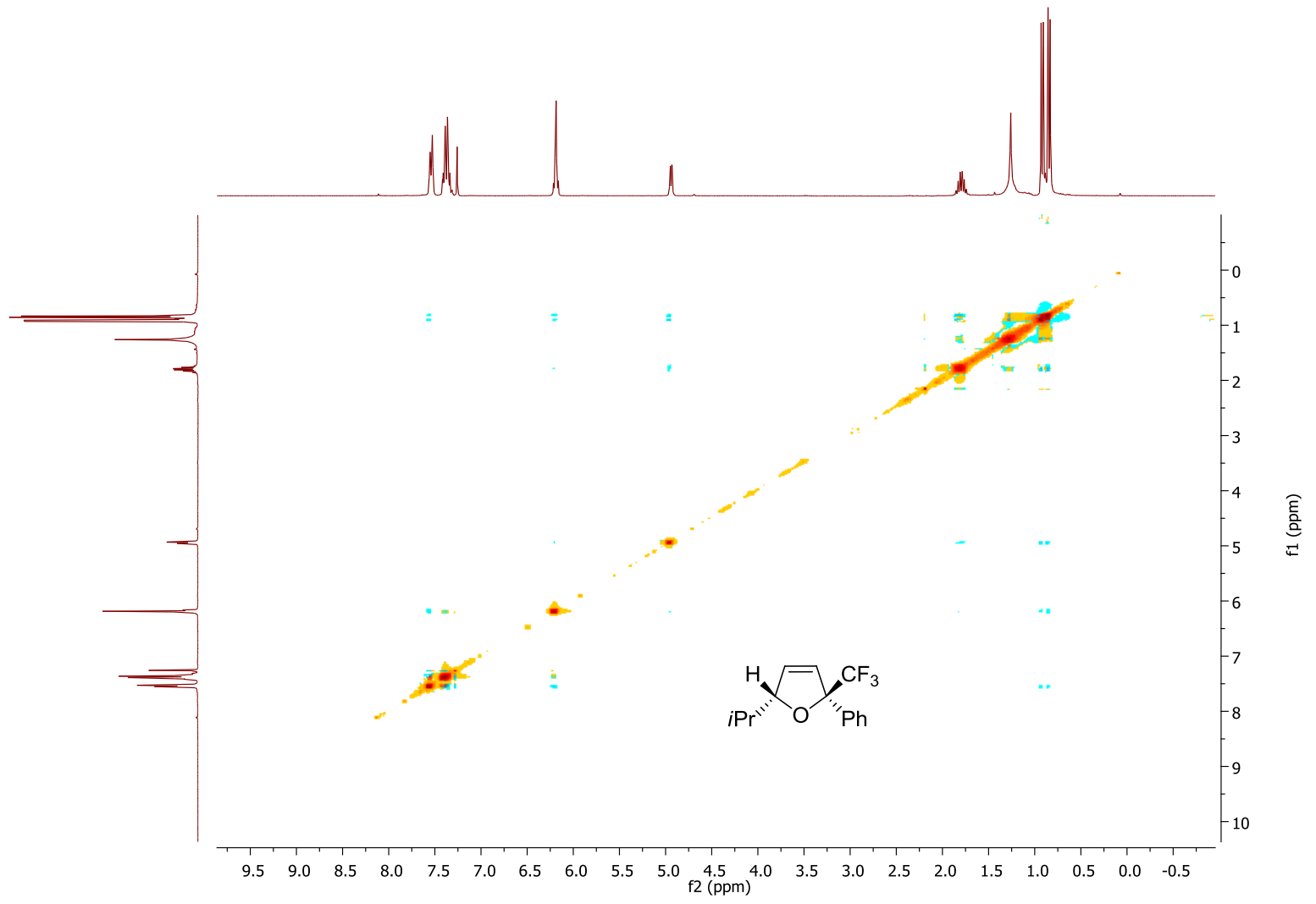




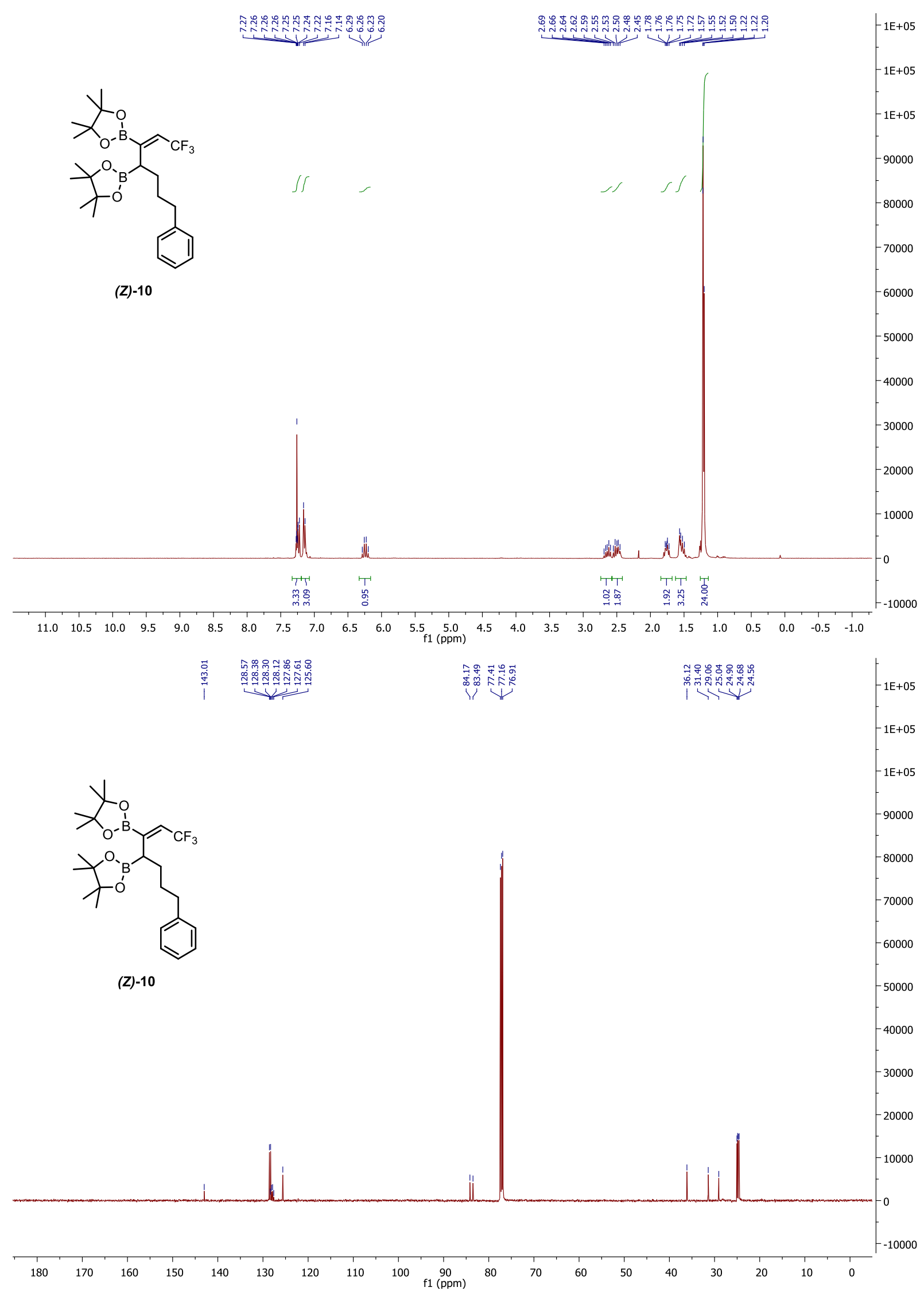




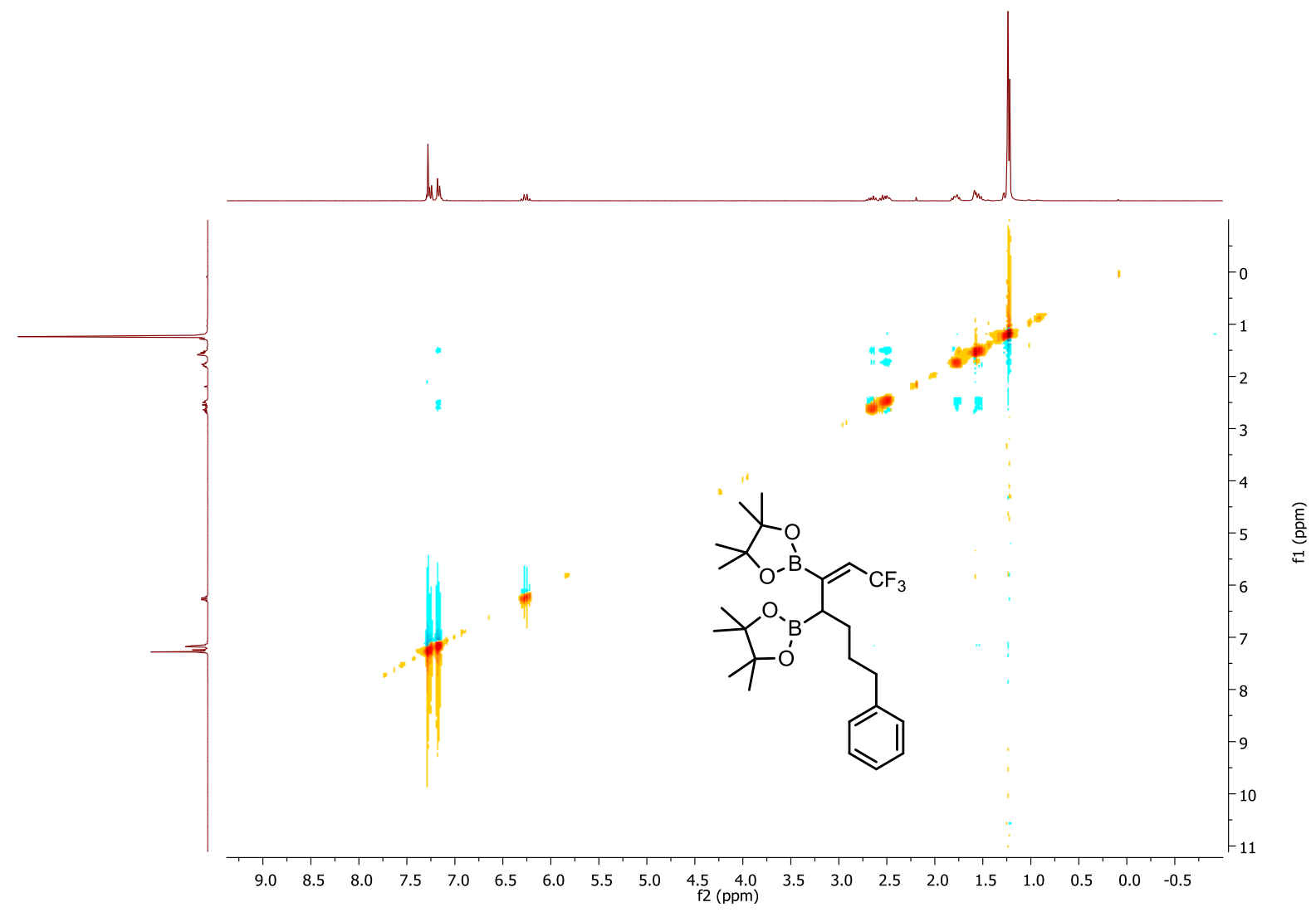




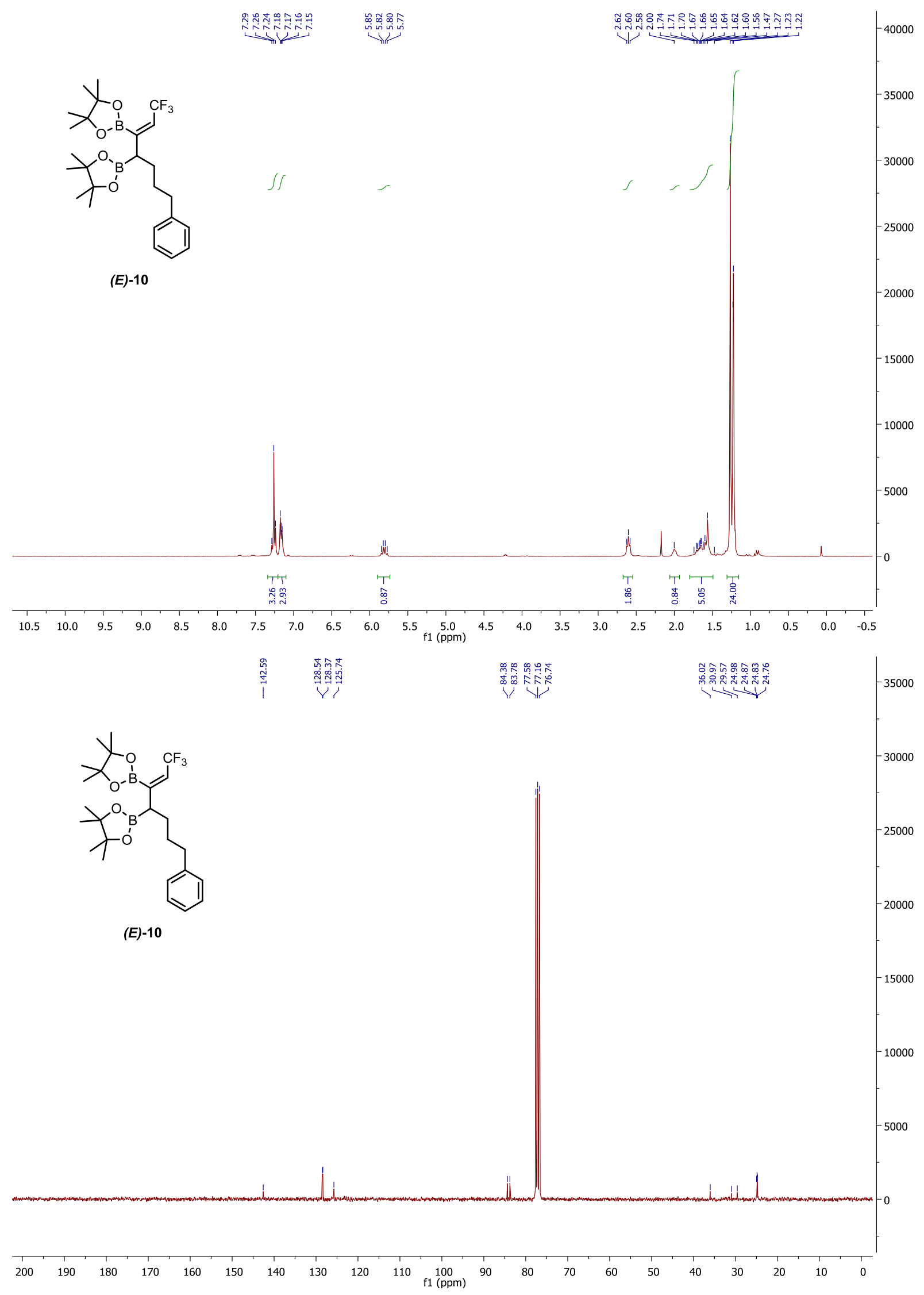




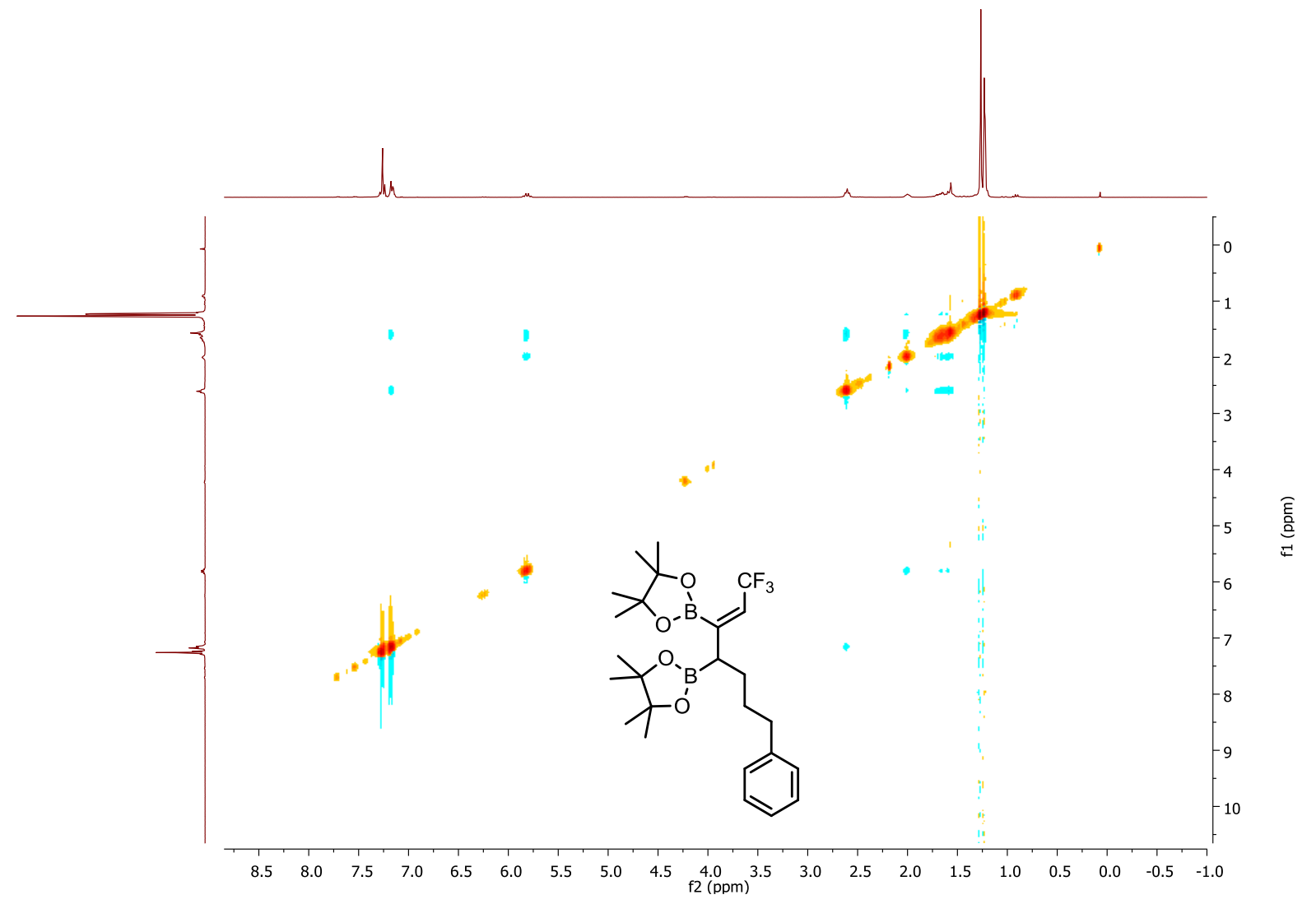




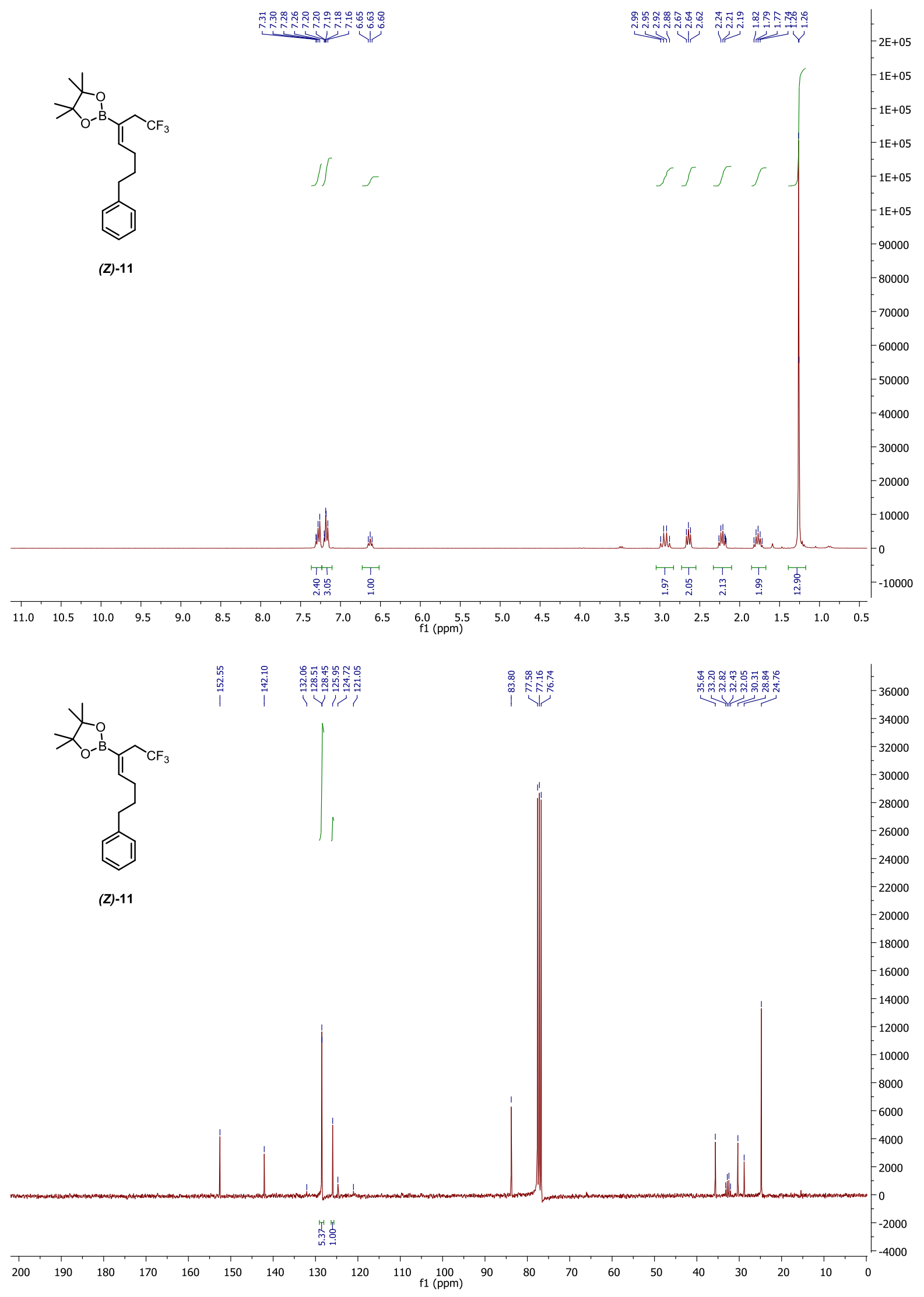




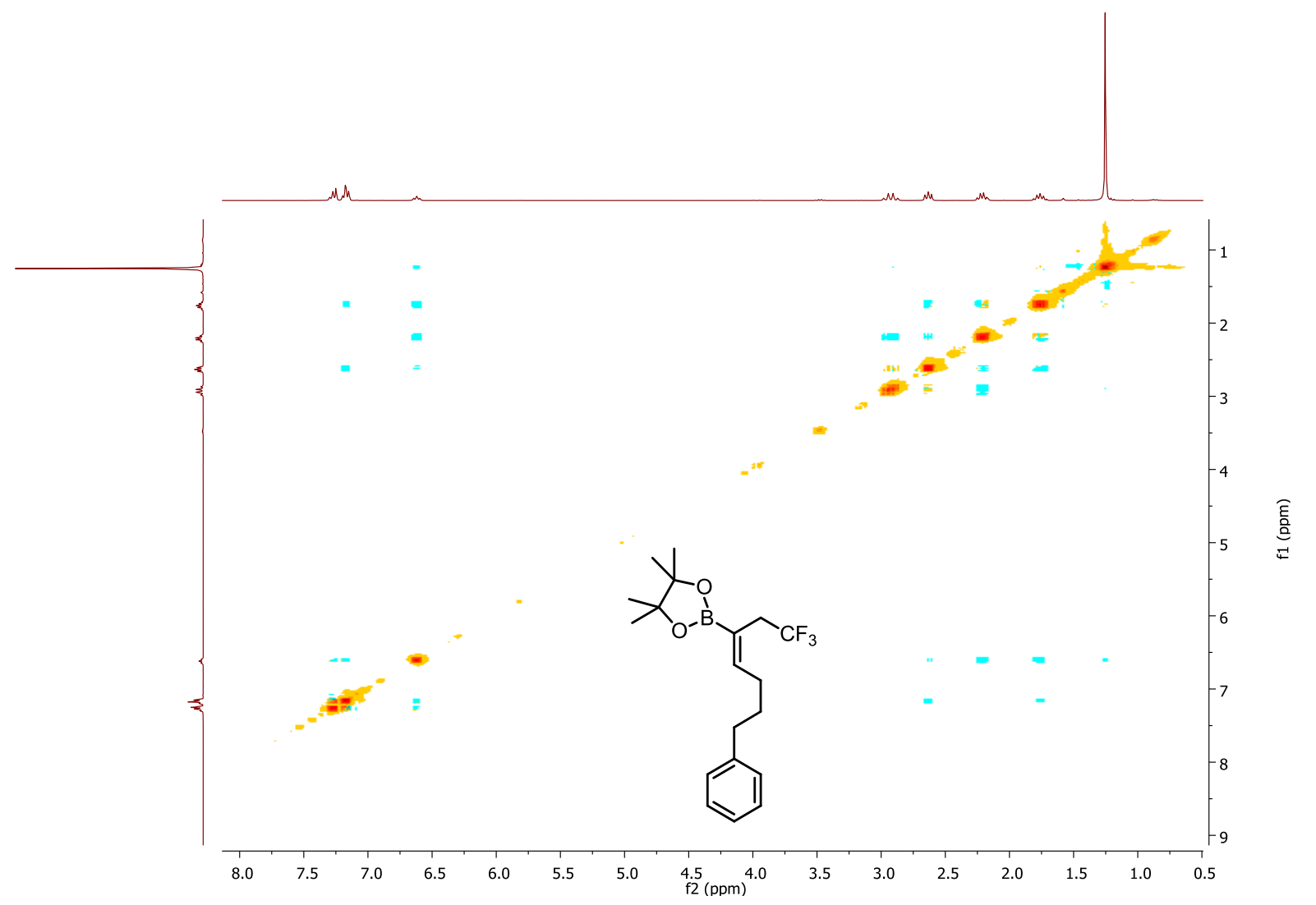




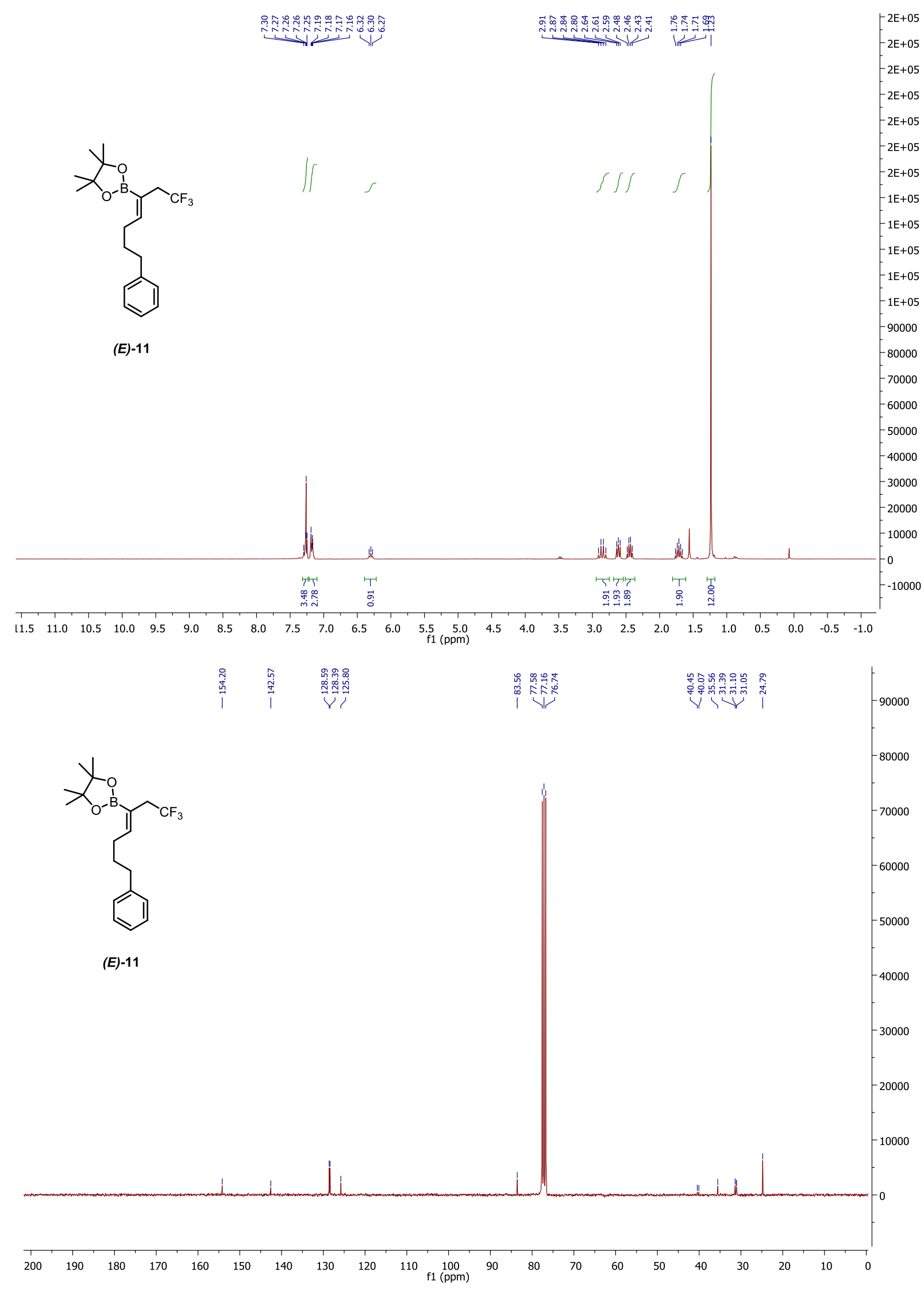




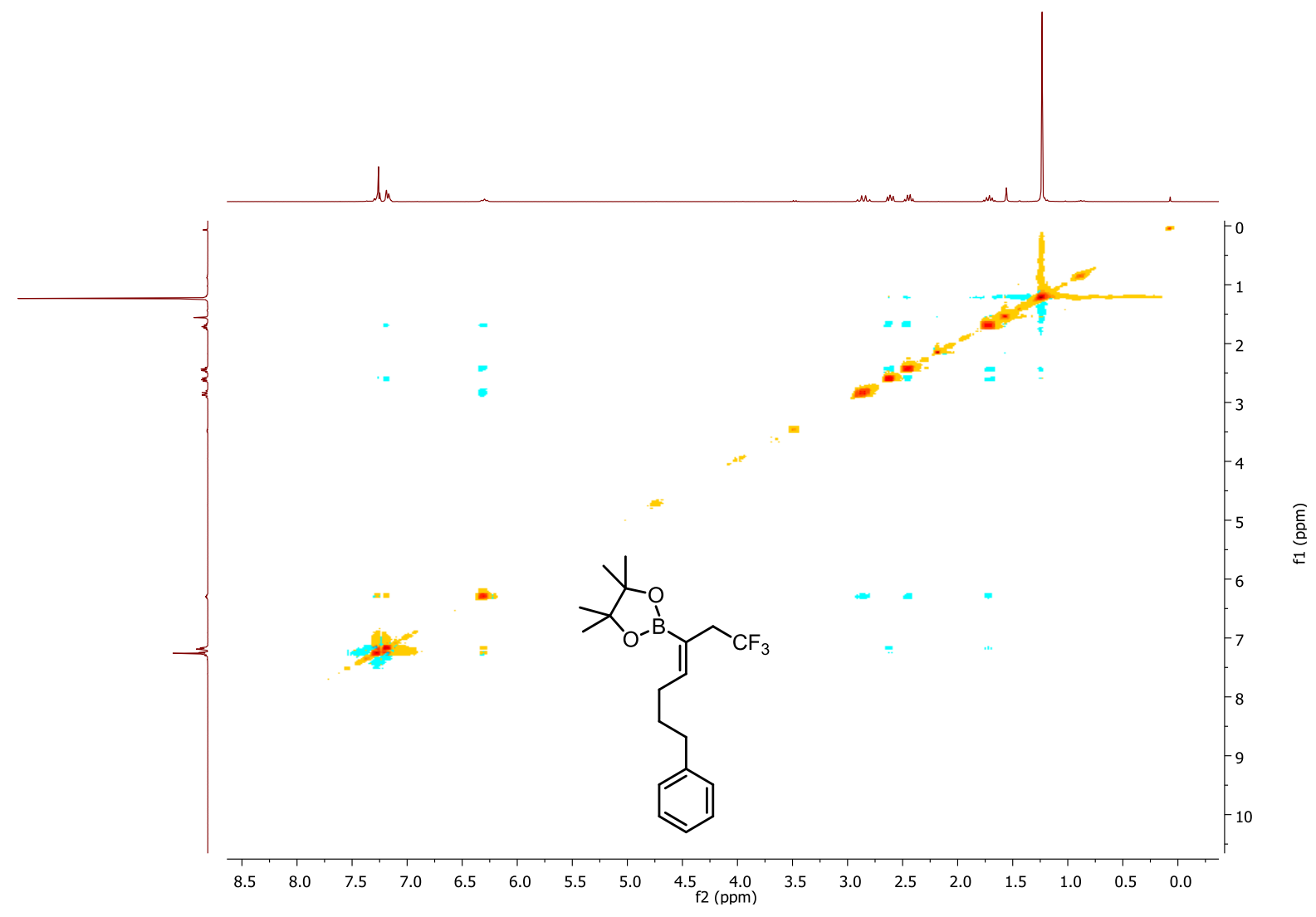

${ }^{1}$ Henrion, G.; Chavas, T. E. J.; Le Goff, X.; Gagosz, F. Angew. Chem. Int. Ed. 2013, 52, 62776282.

${ }^{2}$ Fushibe, K.; Jyono, H.; Fujiwara, M.; Kudo, T.; Yokot, M.; Ichikawa, J. Chem. Eur. J. 2011, $17,12175-12185$.

${ }^{3}$ Boivin, J.; El Kaim, L.; Zard, S. Z. Tetrahedron, 1995, 51, 2573-2584.

${ }^{4}$ With this procedure, the tertiary alcohol disubstituted by the alkyne was targeted. The described alcohol was obtained as a by-product of the reaction but was found to be interesting for the scope of the reaction.

${ }^{5}$ Pelz, N. F.; Woodward, A. R.; Burks, H. E.; Sieber, J. D., Morker, J. P. J. Am. Chem. Soc. 2004, 126, 16328-16329 